\title{
A Bioarchaeological Study of Mid-Holocene Communities in the Eastern Cape, South Africa: the Interface between Foraging and Pastoralism
}

by

Jaime Kristen Ginter

A thesis submitted in conformity with the requirements

for the degree of Doctor of Philosophy

Department of Anthropology

University of Toronto

(C) Copyright by Jaime Kristen Ginter 2008 


\title{
A Bioarchaeological Study of Mid-Holocene Communities in the Eastern Cape, South Africa: the Interface between Foraging and Pastoralism
}

\author{
Jaime Kristen Ginter \\ Doctor of Philosophy \\ Department of Anthropology \\ University of Toronto
}

2008

\section{Abstract}

The late Holocene marks a period of significant population movement and subsistence change throughout much of sub-Saharan Africa. Around 3500 BP it appears that foraging populations in southernmost South Africa began to experience stress related to an increasing population and changing climatic conditions. Approximately 1500 years later a new form of subsistence - sheep herding emerged in areas previously occupied solely by foragers, but was not exclusively adopted. The mechanisms surrounding the introduction of this new subsistence strategy - an indigenous adoption via diffusion or a foreign migration - remain unresolved. This study takes a biological approach to this significant question in southern African prehistory by exploring a collection of Later Stone Age skeletal remains that predate and postdate the appearance of pastoralism in order to determine if any significant changes in skeletal morphology indicative of population discontinuity can be identified at 2000 BP.

A collection of seventy-three Later Stone Age adult skeletons (31 M, $42 \mathrm{~F}$ ) with newly generated radiocarbon dates ranging from $8000 \mathrm{BP}$ to $300 \mathrm{BP}$ (uncalibrated) from the Eastern Cape Province of South Africa forms the basis of this study. Questions surrounding population continuity or discontinuity associated with the advent of sheep herding are investigated by examining metric variables collected from the cranium, post cranial skeleton and dentition, in conjunction with cranial discrete traits. Some changes in skeletal morphology are observed, but the timing, pattern and magnitude of these changes are not consistent with a foreign migration. A reduction in overall skeletal size in the absence of changes in shape corresponds with the period of forager intensification. Body size rebounds at around $2000 \mathrm{BP}$ when evidence for a new form subsistence, sheep herding, is first observed in this region, suggesting that some foragers may have adopted sheep and the herding way of life as a stress relieving mechanism, while others maintained the foraging lifestyle. The timing of the observed changes in skeletal size, the absence of shape changes and the homogeneity in cranial discrete trait frequencies through time argues against the idea that sheep herding was introduced to the Cape region by outsiders. Rather, the findings of the current study suggest sheep herding was an indigenous development among existing foragers. 


\section{Acknowledgments}

There are many people to whom I owe much thanks for their support and assistance during this endeavour. First, I must recognize my doctoral supervisor, Susan Pfeiffer, for the endless support and encouragement that she provided during my tenure as a graduate student at the University of Toronto. Had Susan not been willing to send me to South Africa after the first year of my PhD as a 'research assistant' my doctoral studies would have taken a much different course. Thanks to Susan for giving me the latitude to make my own choices and mistakes, while offering as much guidance and feedback as I needed.

I am very appreciative of the input and suggestions that my doctoral committee members, Max Friesen and Michael Schillaci, provided during the various stages of my dissertation. I would also like to thank my doctoral defense committee members, Paul Sciulli and Michael Chazan for generously offering their time and suggestions. Their comments helped me to improve my dissertation and challenged me to think about my research in different ways.

I am indebted to my statistical support team: Michael Schillaci and Helen Kurki. I am so grateful for the statistical support and advice that they offered, and for their infinite patience. The statistical knowledge that I have gained during this process is thanks to you!

This research would not have been possible without the assistance of the many individuals who facilitated access to the South African skeletal collections: Dr. Johan Binneman and Dr. Lita Webley of the Albany Museum in Grahamstown; Dr. Alan Morris and Caroline Powrie of the Department of Anatomy and Cell Biology at the University of Cape Town; Dr. Judith Sealy of the Department of Archaeology at the University of Cape Town; Nalini Pather and Elija Mofokeng of the Department of Anatomy at Witwatersrand University; Dr. James Brink of the National Museum of Bloemfontein; and Dr. Sarah Wurz of the Iziko Museum of Cape Town. I am grateful to these individuals for being so accommodating and making the research time that I spent with their collections enjoyable and worry free. I owe much thanks to Johan Binneman for helping to broaden my understanding of the landscape and archaeology of the Eastern Cape.

I am very appreciative of those that shared information that was integral to this research. Thanks to Judith Sealy, Susan Pfeiffer and Deano Stynder to access to the radiocarbon dates for the Eastern Cape skeletal material; and to Isabelle Ribot and Alan Morris for access to the comparative African craniometric data. Thanks also to Charles FitzGerald for the methodological and technical assistance with the cervical dental methodology. 
Special thanks to the UCT Archaeology faculty and staff (John Lanham, Tony Manhire, John Parkington, Judy Sealy, and Andy Smith) who helped make my first introduction to South Africa and its amazing prehistory at the Archaeology field school so memorable that I was compelled to change the direction of my doctoral research.

I must pay special thanks to my family for their support and encouragement over the years. To my parents, Gail and Gerry Ginter, I owe so much not only for their unfaltering support and unending interest in my academic career, but also for proving a warm and loving space for me to retire to after a long day of research. Thanks also to my sister, Lindsay Ginter, for her technical assistance with so many aspects of my graduate work.

Thanks to all of my UofT Anthropology friends who provided much needed distractions and an outlet from graduate life. Special acknowledgment goes out to my fellow South African researchers and travelling companions: Helen Kurki and Lesley Harrington.

Last, but not least, I have to thank my husband, André Polsky, for his unending love and support during this experience. Thank-you for enduring the ups and downs of graduate life with me. You provided me the space I needed to pursue my studies, reminded me of my goal and helped me gain perspective when needed, and always had the insight to pull me away from my studies when I needed a break - for this and so much more, I am grateful.

The fieldwork component of this project was made possible by a SSHRC grant awarded to Susan Pfeiffer, a School of Graduate Studies Research Travel Grant, and Department of Anthropology Field Research grants.

This dissertation is dedicated to my grandfather, Herman Rempel, who from a young age challenged me intellectually and helped to cultivate my love of learning and spirit of discovery. A true academic at heart! 


\section{Table of Contents}

List of Tables $\quad$ viii

List of Figures $\quad x$

List of Appendices $\quad$ xii

$\begin{array}{ll}\text { Chapter 1: Introduction } & 1\end{array}$

$\begin{array}{ll}\text { Chapter 2: Theoretical and Methodological Background } & 6\end{array}$

2.1 Why Use Human Remains to Explore this Issue? 11

2.2 Theoretical Basis of this Research 14

2.3 The Physical Geography of the Cape Region of South Africa 18

2.4 Who were the Holocene Foragers of the South African Cape? 23

2.5 Evidence for Intensification of the South African Foraging Lifestyle 25

2.6 Subsistence Changes and Population Movement in sub-Saharan Africa 31

2.7 Evidence for the Emergence of Herding in Southern Africa 34

2.8 Evidence for Migration vs. Diffusion 36

2.8.1 Migration 36

$\begin{array}{lll}2.8 .2 & \text { Diffusion } & 40\end{array}$

2.9 Distinguishing Foragers from Herders $\quad 41$

2.9.1 Occupation Sites $\quad 42$

2.9.2 Burial Sites 44

2.10 Relationships Between Southern African Foragers and Herders: Existing

Beliefs and Evidence 46

2.10.1 Linguistic Evidence $\quad 46$

2.10.2 Skeletal Evidence $\quad 47$

2.10.3 Genetic Evidence 48

2.11 Hypotheses 49

Chapter 3: Materials and Methods

3.1 Materials 51

3.2 Methods $\quad 54$

3.2.1 Data Collection 54

3.2.2 Skeletal Assessment $\quad 55$

3.2.2.1 Age and Sex Estimation $\quad 56$

3.2.2.2 Health, Activity, and Diet 58

3.2.3 Metric Data 59

3.2.3.1 Craniometric Methods $\quad 59$

3.2.3.1.1 Comparative Craniometric Data 60

3.2.3.2 Odontometric Methods 60

3.2.3.2.1 Comparative Odontometric Data 61 
3.2.3.3 Postcranial Metric Methods $\quad 62$

3.2.3.3.1 Comparative Postcranial Metric Data 63

3.2.3.4 Statistical Analysis of Metric Data 63

3.2.3.4.1 Univariate Statistics 63

3.2.3.4.2 Multivariate Statistics 64

3.2.3.4.2.1 Principal Components Analysis 64

3.2.3.4.2.2 Curve Estimation Regression 69

3.2.3.4.3 Population Genetic Analysis 69

3.2.4 Morphological Data $\quad 75$

3.2.4.1 Cranial Discrete Data $\quad 75$

3.2.4.1.1 Comparative Cranial Non-metric Data 76

3.2.4.2 Statistical Analysis of Discrete Data 76

$\begin{array}{ll}\text { Chapter 4: Results } & 79\end{array}$

$\begin{array}{ll}4.1 \text { Craniometrics } & 79\end{array}$

$\begin{array}{ll}\text { 4.1.1 Univariate Analysis } & 79\end{array}$

4.1.2 Multivariate Analysis 81

4.1.2.1 Principal Components Analysis (PCA) 81

4.1.2.2 Curve Estimation Regression $\quad 85$

4.1.3 Comparison with Other Sub-Saharan African Samples 92

4.2 Odontometrics 96

4.2.1 Univariate Analysis 96

4.2.1.1 Maximum Dimensions Method 96

4.2.1.2 Cervical Dimensions Method 98

4.2.2 Multivariate Analysis $\quad 99$

4.2.2.1 Principal Components Analysis of Cervical Dimensions 99

4.2.2.2 Curve Estimation Regression of Cervical Dimensions 104

4.2.3 Comparison with Other South African Samples 110

4.3 Postcranial Measurements 113

$\begin{array}{ll}\text { 4.3.1 Univariate Analysis } & 113\end{array}$

$\begin{array}{lll}\text { 4.3.2 Multivariate Analysis } & 115\end{array}$

4.3.2.1 Principal Components Analysis (PCA) 115

4.3.2.2 Curve Estimation Regression 118

4.3.3 Comparison with Other South African Samples 123

4.4 Summary of Metric Results 126

4.5 Population Genetic Analysis 126

4.6 Cranial Discrete Traits 131

$\begin{array}{ll}\text { 4.6.1 Univariate Analysis } & 131\end{array}$

4.6.2 Comparison with Other South African Samples 137 
Chapter 5: Discussion and Conclusions 139

5.1 Intensification of Foraging Behaviour and Decline in Body Size $\quad 140$

5.2 Was sheep Herding Adopted to Alleviate Population Stress? 147

5.2.1 Skeletal Evidence of Diffusion 148

5.2.2 Insights from the Comparative Analyses 152

5.2.3 Cranial Discrete Trait Frequencies and the Diffusion Hypothesis 155

5.2.4 Changes in Gene Flow and the Diffusion Hypothesis 159

5.3 Variability in the Timing of Changes in Body Size Across the Cape Region 165

5.4 Possible Explanations for the Post-500 BP Increase in Body Size 169

5.5 Chapter Summary and Conclusions 171

Literature Cited $\quad 175$

$\begin{array}{ll}\text { Appendices } & 201\end{array}$ 


\section{List of Tables}

Table 2.1 Variations in Forager Behaviour between 3500 BP and 2000BP 27

Table 2.2 LSA burial practices: forager and herder differences 46

Table 3.1 Distribution of skeletal remains by Institution $\quad 51$

Table 3.2 Metric variables used in the craniometric, odontometric and postcranial $\begin{array}{ll}\text { metric Principal Component analyses. } & 68\end{array}$

Table 3.3 Craniometric variables used in the Relethford-Blangero analysis 73

Table 4.1 Probability values for Craniometric Post-hoc1 ANOVA tests - variables that differ significantly between sub-groups $(p<0.05) \quad 80$

Table 4.2 Craniometric Principal Component Analysis - Variable sets and corresponding Principal Component loadings

Table 4.3 Comparisons between Eastern Cape and Comparative Female Craniometric

Data - t-tests using means and standard deviations

Table 4.4 Comparisons between Eastern Cape and Comparative Male Craniometric

Data - t-tests using means and standard deviations

Table 4.5 Probability values for Maximum Buccolingual Odontometric Dimensions Post-hoc ANOVA tests - variables that differ significantly between sub-groups $(p<0.05)$

Table 4.6 Probability values for Cervical Odontometric Post-hoc ANOVA tests variables that differ significantly between sub-groups $(p<0.05)$

Table 4.7 Cervical Odontometric variable sets and corresponding Principal Component loadings 100

Table 4.8 t-test Comparisons between Eastern Cape and Published Maximum Odontometric data 112

Table 4.9 Probability values for Postcranial Metric Post-hoc ANOVA tests - variables that differ significantly between sub-groups $(p<0.05) \quad 114$

Table 4.10 Postcranial variable sets and corresponding Principal Component loadings 116

Table 4.11 t-test Comparisons between Eastern Cape and Published South African Bantu-speaking Postcranial Data 125

Table 4.12 Relethford and Blangero analysis of gene flow

Table 4.13 Results of multivariate analysis of variance (MANOVA) of the first 8 principal components of the PCA conducted on the cranial variables used in the Relethford-Blangero analysis

Table 4.14 Characteristics of the 16 principal components of the PCA on the cranial variables used in the Relethford-Blangero analysis

Table 4.15 Cranial Discrete Frequency Data

Table 4.16 Results of Pearson chi-square analysis of Eastern Cape male and female cranial discrete trait frequencies

Table 4.17 Results of Pearson chi-square analysis of Pre- and Post-2000 BP cranial discrete trait frequencies 
Table 4.18 Results of Pearson chi-square analysis of Eastern Cape and comparative South African Bantu-speaking cranial discrete trait frequencies 


\section{List of Figures}

Figure 2.1. Migration route sheep herders as proposed by Elphick (1977) 9

Figure 2.2. Biomes of southern Africa. Eastern Cape study area indicated 21

Figure 4.1. Scatterplot of PC1 and PC2 scores for vault variables 82

Figure 4.2. Scatterplot of PC1 and PC2 scores for face variables 83

Figure 4.3. Scatterplot of PC1 and PC3 scores for face variables 83

Figure 4.4. Scatterplot of PC1 and PC2 scores for mandibular variables 85

Figure 4.5. Scatterplot of regression scores for vault variables: PC1 vs. ${ }^{14} \mathrm{C} \quad 86$

Figure 4.6. Scatterplot of regression scores for vault variables: PC2 vs. ${ }^{14} \mathrm{C}$

Figure 4.7. Scatterplot of regression scores for face variables: PC1 vs. ${ }^{14} \mathrm{C}$

Figure 4.8. Scatterplot of regression scores for face variables: PC2 vs. ${ }^{14} \mathrm{C}$

Figure 4.9. Scatterplot of regression scores for face variables: PC3 vs. ${ }^{14} \mathrm{C}$

Figure 4.10. Scatterplot of regression scores for mandibular variables: PC1 vs. ${ }^{14} \mathrm{C} \quad 90$

Figure 4.11. Scatterplot of regression scores for mandibular variables: PC2 vs. ${ }^{14} \mathrm{C} \quad 91$

Figure 4.12. Scatterplot of PC1 and PC2 scores for maxillary anterior cervical variables 102

Figure 4.13. Scatterplot of PC1 and PC2 scores for maxillary posterior cervical variable 102

Figure 4.14. Scatterplot of PC1 and PC2 scores for mandibular anterior cervical variables 103

Figure 4.15. Scatterplot of PC1 and PC2 scores for mandibular posterior cervical variables 103

Figure 4.16. Scatterplot of PC1 and PC3 scores for mandibular posterior cervical variables $\quad 104$

Figure 4.17. Scatterplot of regression scores for maxillary anterior cervical variables: PC1 vs. ${ }^{14} \mathrm{C} \quad 105$

Figure 4.18. Scatterplot of regression scores for maxillary anterior cervical variables: PC2 vs. ${ }^{14} \mathrm{C} \quad 106$

Figure 4.19. Scatterplot of regression scores for maxillary posterior cervical variables: PC1 vs. ${ }^{14} \mathrm{C} \quad 106$

Figure 4.20. Scatterplot of regression scores for maxillary posterior cervical variables: PC2 vs. ${ }^{14} \mathrm{C} \quad 107$

Figure 4.21. Scatterplot of regression scores for mandibular anterior cervical variables: PC1 vs. ${ }^{14} \mathrm{C} \quad 108$

Figure 4.22. Scatterplot of regression scores for mandibular anterior cervical variables: PC2 vs. ${ }^{14} \mathrm{C} \quad 108$

Figure 4.23. Scatterplot of regression scores for mandibular posterior cervical variables: PC1 vs. ${ }^{14} \mathrm{C} \quad 109$

Figure 4.24. Scatterplot of regression scores for mandibular posterior cervical variables: PC2 vs. ${ }^{14} \mathrm{C} \quad 109$

Figure 4.25. Scatterplot of regression scores for mandibular posterior cervical variables: PC3 vs. ${ }^{14} \mathrm{C} \quad 110$

Figure 4.26. Scatterplot of PC1 and PC2 scores for the trunk variables 117

Figure 4.27. Scatterplot of PC1 and PC2 scores for the upper limb variables 117

Figure 4.28. Scatterplot of PC1 and PC2 scores for the lower limb variables 118

Figure 4.29. Scatterplot of regression scores for trunk variables: PC1 vs. ${ }^{14} \mathrm{C} \quad 119$

Figure 4.30. Scatterplot of regression scores for trunk variables: PC2 vs. ${ }^{14} \mathrm{C} \quad 120$

Figure 4.31. Scatterplot of regression scores for upper limb variables: PC1 vs. ${ }^{14} \mathrm{C} \quad 121$

Figure 4.32. Scatterplot of regression scores for upper limb variables: PC2 vs. ${ }^{14} \mathrm{C}$

Figure 4.33. Scatterplot of regression scores for lower limb variables: PC1 vs. ${ }^{14} \mathrm{C} \quad 122$

Figure 4.34. Scatterplot of regression scores for lower limb variables: PC2 vs. ${ }^{14} \mathrm{C} \quad 123$ 
Figure 4.35. The impact of effective population size estimates on estimates of gene flow

Figure 4.36. Plot of PC1 and PC2 for the PCA of the Relethford-Blangero craniometric variables

Figure 4.37 Distribution of the os japonicum discrete trait in Eastern Cape sample 


\section{List of Appendices}

Appendix 1. Map of the Eastern Cape Region displaying some of the burial locations of the skeletal remains studied in this research

201

Appendix 2. Total Eastern Cape Adult Skeletal Sample

202

Appendix 3. List of Craniometric Variables Examined in this Study

Appendix 4. List of Odontometric Variables (Maximum and Cervical Dimensions) Examined in this Study

Appendix 5. List of Postcranial Variables Examined in this Study

Appendix 6. Dichotomous Cranial Non-metric Traits Used in Analysis

206

Appendix 7. Craniometric Summary Data $(\mathrm{mm})$

207

Appendix 8. Maximum Odontometric Dimensions Summary Data ( $\mathrm{mm})$

Appendix 9. Cervical Odontometric Dimensions Summary Data ( $\mathrm{mm}$ )

213

Appendix 10. Postcranial Metric Summary Data (mm)

216

Appendix 11. Kolmogorov-Smirnov test of normality for craniometric variables with Monte

220 Carlo approximations

Appendix 12. Kolmogorov-Smirnov test of normality for maximum craniometric variables with Monte Carlo approximations

Appendix 13. Kolmogorov-Smirnov test of normality for cervical odontometric variables with Monte Carlo approximations

Appendix 14. Kolmogorov-Smirnov test of normality for postcranial metric variables with Monte Carlo approximations

Appendix 15. Levene's test for homogeneity of variances for the craniometric variable set

Appendix 16. Levene's test for homogeneity of variances for the maximum odontometric variable set

Appendix 17. Levene's test for homogeneity of variances for the cervical odontometric variable set

Appendix 18. Levene's test for homogeneity of variances for the postcranial metric variable set 


\section{Chapter 1 - Introduction}

The forces motivating culture and subsistence change are vast and complex. Population replacement and human migration have served as the impetus for socioeconomic change both historically and prehistorically, but are not requisite. In other cases, cultural modification and development has been indigenous, stimulated by environmental factors. Questions relating to prehistoric subsistence change can be adequately studied in southern Africa. In South Africa, the transition from foraging to herding has long been debated in the context of population continuity or replacement. Foraging dominated southern African subsistence for most of prehistory. Foraging was the first and most longstanding form of subsistence, existing exclusively in this region for 20, 000 years (Deacon and Deacon 1999; Phillipson 2005), but it was not static. Throughout the Holocene there is evidence of changing patterns of occupation and alterations to traditional foraging behaviour. Cultural and genetic continuity was maintained during these periods of flux, suggesting changing climatic and environmental conditions as the most likely cause (cf. Mitchell 2001). It is not until around 2000 years ago that evidence for that new subsistence approach - sheep herding - emerges. What makes the question of the origin of sheep herding so complex is that the transition to herding did not signal the end of foraging. Rather, the foraging lifestyle continued to exist alongside herding for many centuries. The appearance of more substantial changes in the material culture contemporary with evidence of sheep herding around 2000 BP, prompted many scholars to attribute the emergence of sheep herding in South Africa to the migration of herding groups from north of the Zambezi River. Others assert that herding was an in-situ development among some indigenous foraging groups whereby some groups adopted herding while others maintained foraging alone. 
The mechanisms surrounding the arrival of sheep herding have been debated and explored through approaches from archaeology, linguistics, genetics, and ethnology, yet the factor(s) responsible for the introduction of this new way of life - a migration of peoples or a diffusion of ideas - continue to be debated. Although differences in archaeological evidence for foragers and herders have been proposed (Smith et al. 1991), their social and cultural similarities have made it difficult to resolve this issue. The potential that information gleaned from the study of the human skeletal remains of the foragers and pastoralists themselves could add to this question has not been fully realized.

This study attempts to explore the origins of sheep herding in South Africa and the mechanisms responsible for the emergence of this new subsistence strategy by exploring the skeletal morphology of a relatively unstudied sample of Later Stone Age (LSA) adult skeletal remains from the Eastern Cape region of South Africa. This issue will be approached from a bioarchaeological perspective, incorporating information obtained from the study of human remains with additional lines of evidence. Archaeological, paleoenvironmental, zooarchaeological, ethnographic, and genetic information will be consulted to help situate the information obtained from the study of the human skeletal remains in time and space. To provide input to the debate over the arrival of herding, genetic and behavioural skeletal data were gathered. Craniometric, odontometric and post cranial metric data were collected in conjunction with cranial discrete data. Evidence of population continuity or replacement will be ascertained through the exploration of potential differences in size, shape, and discrete trait frequencies among the inhabitants of the Eastern Cape through time.

One of the main obstacles to this research lies in distinguishing between difference and variation. Since the objective of this research is to explore whether the emergence of 
sheep herding in southern South Africa was the result of a foreign introduction or an indigenous development, it is necessary to distinguish skeletally between skeletal evidence of variation within an existing population and difference possibly associated with an influx of new genes. Variations in the environment, climate, vegetation, and topography, and behaviour will be manifest as differences in skeletal morphology. The identification of regional differences in adult human bone mass and limb strength as indicators of habitual activity (Pfeiffer and Stock 2002; Stock 2002), body size and stature (Pfeiffer and Sealy 2006), positional behaviour (Dewar and Pfeiffer 2004), and trauma (Pfeiffer 2001) indicative of subsistence shifts, among southern and western Cape Later Stone Age (LSA) populations supports this premise. Based on these findings, it is possible to propose that evidence of migration and diffusion can be accessed in the human skeleton. Minor to moderate differences in skeletal size in conjunction with similarities in cranial discrete frequencies through time will be interpreted to reflect within-group variability resulting from environmental factors. In comparison, marked differences in skeletal size and changes to skeletal shape, along with differences in cranial discrete trait frequencies through time will be taken to reflect genetic change.

The subsequent chapters detail how this research was undertaken. The second chapter explores the theoretical and methodological basis of this research. The benefits of studying the questions relating to prehistoric subsistence change, like that undertaken herein for the introduction of sheep herding in the Eastern Cape, are discussed. The theoretical principals that guide the analysis and interpretation of the skeletal data are discussed in the context of forager adaptation, variability and agency. The prehistory of South African forager occupation is discussed, along with a synthesis of the history of the foraging way of 
life throughout the Holocene. The two central premises concerning the mechanisms responsible for the introduction of sheep herding are explored and the evidence in support of migration and diffusion are compared. Attempts that have been made to distinguish foragers from herders based on burial and occupation information are discussed along with the existing evidence for the relationship between southern African foragers and herders. The second chapter ends with a brief discussion of the three hypotheses that govern this research.

Chapter three outlines the data collection methods that were used and provides details of the skeletal sample under study. The metric methods that were employed in the metric data collection are discussed individually for each anatomical region of the skeleton: cranium, dentition, and postcranial skeleton. The univariate and multivariate statistical methods that were employed to analyze the metric data are explained. The chapter concludes with a discussion of the methodological approach to the collection and analysis of the cranial discrete data. The comparative data available for the metric and morphological analysis are explored in turn.

The various results of the metric and morphological study of the Eastern Cape sample are provided in Chapter four. The results of the various analyses are organized in the same fashion as in the previous chapter. The univariate and multivariate findings are presented individually for each of the skeletal anatomical regions studied. A discussion of the results of the cranial discrete trait analysis closes the chapter.

A synthesis of the craniometric, odontometric, postcranial metric, and cranial discrete trait results is presented in the fifth and final chapter. The implications of the findings for the hypotheses concerning the introduction of sheep herding are discussed. This dissertation 
concludes with a summary of the research findings and the implications of the results for current understandings of South African prehistory. 


\section{Chapter 2 - Theoretical and Methodological Background}

Population movement, contact and adaptation have served as the catalysts of hominin evolution and the development of human species. African populations exhibit the greatest within-group variance and diversity of all the world's populations (cf. Cavalli-Sforza et al.

1994). The great antiquity of modern human occupation on the African continent contributes significantly, but not exclusively, to this diversity. Variation in modern human morphology has typically been attributed to gene flow. But is population movement requisite for morphological and cultural change? Can smaller modifications on a regional scale serve as an adequate stimulus to alter behavior and biology? To understand modern human diversity, all of the evolutionary processes that have contributed to development of different regional or group morphologies - genetic, behavioural, and environmental - should be explored.

Geographical expansion not only put groups in contact with new environments but also with other groups, leading to adaptation to new environs, the transmission and adoption of new ideas, and potentially the assimilation or displacement of indigenous groups.

The period around $2000 \mathrm{BP}$ is associated with cultural change, subsistence shifts, and human dispersals across sub-Saharan Africa. This period of change was set in motion by a dispersal event that began around 3000 years ago when Bantu-speaking agricultural groups began a southward migration from West-Central Africa eventually reaching the south-eastern regions of South Africa 1500 years later. The events surrounding this agricultural exodus and its effects on these immigrant groups have been extensively studied. The impact of this large scale population expansion into regions previously occupied exclusively by foragers and the implications of this upheaval on the biology and culture of the non-Bantu-speaking groups is underappreciated. 
The research presented herein explores issues relating to socioeconomic change among populations inhabiting southern Africa during the Holocene. The mechanisms responsible for subsistence change are explored in the context of the arrival of sheep herding in areas of South Africa previously occupied exclusively by foragers. Questions relating to prehistoric subsistence variability can be effectively studied in southern Africa, an area with a long history of human occupation and relatively high archaeological visibility that can be partially attributed to an environment that promotes excellent preservation. The transition from foraging to herding is particularly accessible in South Africa where the excellent archaeological preservation and rich cultural record has allows the exploration of many questions relating to health, subsistence and lifestyle in a way that is not possible in other areas of the world. The complexity of Southern Africa's settlement history is unique. In this area, the new subsistence lifestyle of herding did not completely replace the existing way of life. Foraging continued to exist alongside herding, unlike Europe and the Middle East where the hunting and gathering way of life terminated with the arrival of domesticates during the Neolithic. The incomplete nature of this subsistence transition has made determining the origins of the herders and their relationship to the foragers problematic. The timing of the arrival of the keeping of sheep to southernmost South Africa at around 2000 B.P is generally agreed upon, yet the mechanisms responsible for this subsistence shift - a migration of peoples or a diffusion of ideas - remains open to debate. The most prominent theory, based on ethnographic, archaeological and linguistic information, suggests that herding groups migrated to South Africa from the north of the Zambezi River around 2000BP (cf. Boonzaier et al. 1996; Deacon and Deacon 1999; Smith et al. 2000) (Figure 2.1). However, Sadr (2003) and a growing number of academics are citing recent 
excavations and reanalyses of archaeological data that suggest that a Neolithic Transition occurred whereby the idea of herding and the sheep, rather than herders themselves, spread throughout South Africa. This led some foragers to make the transition to herding while others maintained their forager lifestyle. The speed at which herding traveled from the north to the southern part of the continent, as attested by a relative lack of variability in dates for sites that sometimes range over distances of up to 5000 kilometers (Deacon 1984; Kinnahan 1996), is puzzling. Although differences in archaeological evidence for foragers and herders have been proposed (cf. Boonzaier et al. 1996; Smith et al. 2000), their social and cultural similarities, as well as the fluidity of the respective lifestyles (Kelly 1995; Kusimba 2002), have made this chapter of South Africa's prehistory difficult to resolve. Evidence of cultural and economic change is hard to track among more mobile groups, like foragers and herders. Because of their lifestyle and subsistence practices, a visible footprint is not always left on the spaces they occupy. The complexity of this issue demands that research move beyond the more traditional realms of inquiry of archaeology, ethnography, and linguistics to explore additional lines of evidence in hopes achieving clarity for this longstanding population interaction question

This research takes a biological, specifically a bioarchaeological, approach to the question of herder and forager relationships by exploring similarities and differences in the skeletal remains of the individuals themselves in conjunction with information from the broader archaeological context. The analytic approaches employed by bioarchaeologists 
Figure 2.1. Migration route of sheep herders as proposed by Elphick (1977). (Source: Elphick 1977)

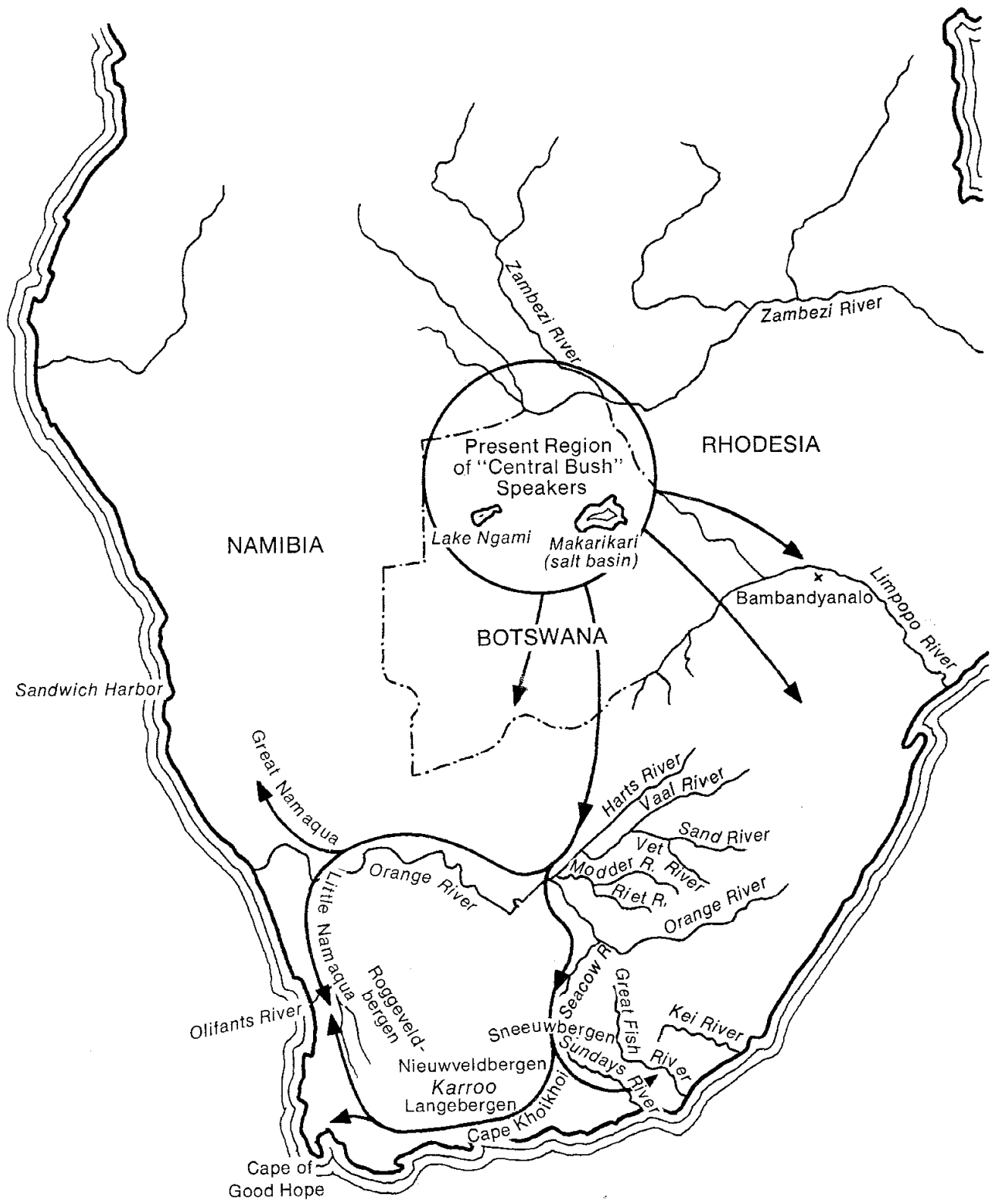

Key: $-\cdot-\cdot-$ Boundaries of Botswana

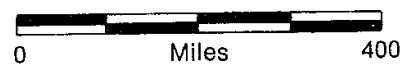


enable information about biology, culture and the environment to be extracted from the skeletal remains. The human skeleton serves as an excellent avenue for investigating questions about population interaction and subsistence change. Information about lifestyle and subsistence, including growth and development, diet, health and physical activity are reflected in the morphology of the human skeleton (cf. Larson 1997). Both behavioural and genetic information are recorded in the human skeleton. Similarities and differences in skeletal morphology can be explored within and between populations across time and space to help inform about questions of biological relatedness, population continuity and cultural change, issues that are at the centre of the southern African forager-herder debate. This research aims to not only contribute to the ongoing 'introduction of herding' debate, but also to add much needed information about the biology of the prehistoric Later Stone Age (LSA) inhabitants of Eastern Cape, an area of South Africa that remains understudied.

To provide input to the debate over the arrival of herding, genetic and behavioral information were gathered from a relatively unstudied sample of Later Stone Age (LSA) skeletal remains from the Eastern Cape region of South Africa. This study is the first to take a complete skeletal approach to the investigation of evidence of skeletal change at around 2000 BP. Any observed changes in skeletal size and shape among inhabitants of the Eastern Cape through time will be assessed from metric and morphological data gathered from cranial and postcranial remains. The nature of skeletal change can inform about the factors responsible for any observed alterations to skeletal morphology concurrent with the initial appearance of domestic stock: intrinsic (gene flow, genetic drift, etc.) or extrinsic (environment, stress, diet, etc.). The pattern and magnitude of any observed changes in skeletal morphology at around $2000 \mathrm{BP}$ will be used to assess population continuity 
(diffusion) or discontinuity (migration) among the groups inhabiting the Eastern Cape across the LSA. Supporting information in the form of archaeological, ethnographic, linguistic, genetic, and palaeoenvironmetal data are used to further substantiate the results of this research. Through an exploration of the socioeconomic status of other sub-Saharan populations during this period in the Holocene and the impact that contact between and among groups had on the trajectory of southern African populations, it will be possible to postulate the population dynamics associated with subsistence change in southernmost South Africa.

\subsection{Why use Human Remains to Explore this Issue?}

As anthropologists, our chief interest is discovering information about the lifeways of different cultural groups. The nature and antiquity of the cultural group under study dictates the methods that can be of use in gathering such data. Biological anthropologists, and more particularly bioarchaeologists who deal with prehistoric human populations, can access details about the culture and behavior of an individual or group that are not easily ascertained by other means. Because aspects of an individual's life are preserved within the skeleton, researchers are able to interpret various aspects of human life, such as diet and subsistence practices, health, body size, activity, and population demography (cf. Blakely 1977; Cohen and Armelagos 1984; Cohen 1989; Powell et al. 1991; Larsen 1997; Steckel and Rose 2002; Williamson and Pfeiffer 2003; Buikstra and Beck 2006). Uncovering information about an individual's or group's lifestyle helps to fill in one more piece of the complex puzzle of prehistoric human behavior. Because bones and teeth preserve biologically relevant information about the past (Larsen 1997:2) human skeletal remains can, in conjunction with other lines of evidence, provide a valuable source of information that enables researchers to 
interpret various aspects of past human life. In order to fully appreciate the complex web of factors that contribute to and shape prehistoric human behavior, information from a number of sources including archaeology, ethnoarchaeology, linguistics, genetics, and environmental science must be integrated.

Stress, resulting from social and economic change associated with the shift to a new way of life, can manifest on the skeleton as growth disruption, disease or illness, or ultimately death. Studies that have explored the impact of the transition from foraging to agriculture on the human skeleton, have found that differences in diet, mobility, subsistence technology, and activity affect the growth and development of the skeleton in a variety of ways (cf. Blakely 1977; Boyd and Boyd 1989; Cohen and Armelagos 1984; Cohen 1989; Powell et al. 1991; Larsen 1995, 1997; Steckel and Rose 2002). Yet, few investigations have examined the effects on human health when the transition was made from foraging to herding. It is reasonable to propose that subsistence shifts from foraging to herding may also be visible in the skeleton. Regional and temporal differences in bone mass and upper arm asymmetry (Pfeiffer and Stock 2002; Stock and Pfeiffer 2004), body size and stature (Sealy and Pfeiffer 2000; Pfeiffer and Sealy 2006), positional behaviour (Dewar and Pfeiffer 2004), and trauma (Pfeiffer in press, 2001) have been identified among Later Stone Age (Holocene) forager populations from the southern and western Cape regions of South Africa. These differences, while not framed in terms of the introduction of herding, are all potentially indicative of subsistence shifts, and add credence to the approach undertaken herein for examining the introduction of herding in southern Africa through the bioarchaeological study of LSA populations from the Eastern Cape region. 
Since genetic information demonstrates that San foragers and Khoekhoe herders share the same ancient Southern African heritage (Chen et al. 2000), it can be hypothesized that lifestyle differences are manifested as morphological differences in the skeleton, and thus could be used to distinguish herders from foragers. While helpful, genetic studies alone cannot help to resolve the debate regarding the introduction of herding. The African genome is the most diverse of all the global populations (cf. Jorde et al. 2000; Gabriel et al. 2002,). Mechanisms such as genetic drift, population bottlenecks and founder effects do not exclusively shape the genetic blueprint of a particular population. Furthermore, genetic studies are primarily conducted on modern or historic populations who may not reflect the genetic diversity or homogeneity of their ancestral forager groups, considering that relationships between modern and historic Khoesan, Bantu-speaking agricultural, and European groups were variable across the region and had differential effects on the culture and biology of the groups involved. Rather, other factors, including climate and environment, have been found to not only contribute to the genetic blueprint (Lahr and Foley 1998), but also affect our ability to detect genetic factors (Cooper et al. 2000). Recent studies have shown that migration and different environments affect allele frequencies in groups with a shared genetic background (Cooper et al. 2000), thus illustrating the difficulty that may be encountered in utilizing genetic information to determine inheritance for groups that share a common ancestry but at some point in time were separated by distance.

By examining both metric and discrete variables, behavioral and genetic information from the skeleton can be accessed, because differences in the size, shape, and morphology of the human skeleton are influenced by both genetic and cultural factors. The main challenge of studies involving osteometric and morphological research is the identification of the extent 
to which these genetic and behavioural factors differentially influence the development of the skeleton. Because the aim of this research is to determine whether the transition to herding was brought about by migration or diffusion, its design reflects a preference for osteometric and morphological variables that have demonstrated both genetic and behavioural meaning. Although environmental factors are known to have some influence on cranial size and shape (cf. Guglielmino-Matessi et al. 1979; Beals et al. 1983; Franciscus and Long 1991; Smith et al. 2007), similarities in cranial form are generally assumed to reflect common biological affinity (cf. Howells 1995; Lahr 1996; Sparks and Jantz 2002; Relethford 2004; Roseman and Weaver 2004). Cranial and dental remains are not as directly affected by behaviour and activity (as compared to the postcranial skeleton) so they should preserve genetic information more directly. The postcranial skeleton is more subject to environmental influences and mechanical factors resulting from mobility and activity (Bogin and Loucky 1997; Pearson 2000; Bogin et al. 2002). Nevertheless there is a genetic component to body size and physique (Trinkaus et al. 1994; Bogin 1999). Skeletal discrete traits have been found to be relatively un-affected by external factors (Berry and Berry 1967; Anderson 1968; Berry 1968; Molto 1983; Hauser and De Stefano 1989; Hanihara et al. 2003). Unlike osteometric measurements, discrete traits can be observed on incomplete and fragmentary skeletal remains, making this a favored method of analysis for investigating topics of biological affinity and population relationships in archaeologically derived skeletal samples (cf. Ossenburg 1978; Molto 1983; De Laurier and Spence 2003; Rubini et al. 2006).

\subsection{Theoretical Basis of this Research}

The continued existence of the foraging way of life is a testament to the fluidity, flexibility, and adaptability embodied in this economic system. Contemporary, historic, and 
prehistoric foragers have faced numerous challenges, ranging from climatic and environmental changes, to dealing with novel regional and inter-regional contact with neighbours. The ever changing physical and social realm in which foragers are situated is constantly in flux, and these groups have actively adapted their socio-economic systems to deal with such situations and ensure continued population success.

Issues of collective human intention and choice must be considered in any discussion involving subsistence and social change. For years many theoretical approaches to the study of foraging societies from both an ethnographic and archaeological perspective, have characterized foraging populations as a people on the periphery or 'on the edge', their very existence dependent on the whims of external social and environmental factors. Studies of prehistoric foraging groups have tended to situate the individual or group in a broader ecological or environmental framework, interpreting the archaeological record and material culture from the perspective that environmental factors shape socio-economic behaviours and are responsible for variability among foraging groups (Winterhalder and Smith 1981; Netting 1986; Smith and Winterhalder 1992; Kelley 1995). While the environment plays a major role, climate and environment are not the sole motivators behind social change. Group decisions and actions are not bounded by particular rules, but are rather informed by various social realities as well as the external environment. New research and re-analyses of existing data through a new, more holistic lens are becoming ever more cognizant of the control that foraging groups have, and the way they exert that control over their social and economic circumstances.

Studies of contemporary southern African foragers, like those of Lee (1993) and Widlock (1999), illustrate the cultural complexity and flexibility of the hunting and gathering 
way of life. Lee (1993) recounts the transitory nature of the Dobe Ju/'hoansi's subsistence through time, moving between foraging, farming and herding when the opportunity and need occurred, all the while maintaining the basic core of a foraging society. Widlok's experience with the Hai//om provides insights into the flexible nature of the foraging social system:

“Contact with neighbouring groups has changed hunting and gathering, but it has not turned the Hai //om into herders or cultivators. While there is flexibility in the sense that hunting and gathering has been adapted to meet the new conditions, there is also stability and continuity at the level of the social relations governing the economy ... This relative inertia cannot be accounted for simply by reference to external factors such as a constraining ecology or the presence of dominant neighbours. A comparison with other 'Bushmen', especially the !Kung, is instructive because it shows a spectrum of social strategies developed under constraints which are not only ecological but also social in nature.” (Widlok 1999:62)

Social agency theory posits that individuals and groups make decisions based on their sociocultural and environmental surroundings (Dobres 2000). In effect, the social agency paradigm sits in opposition to the way that foragers have traditionally been viewed. It regards foragers as the agents who consider various aspects of their surroundings and make decisions about behaviour, rather than having the environment and other external factors determine their actions and behaviours. Social agency theory is predicated on the belief that social relations, as opposed to inanimate external forces, influence the maintenance and alteration of subsistence behaviours. Thus, many factors in the social realm, such as needs or desires to exert markers of group identity, and contact with other subsistence groups, as well 
as the environmental sphere, like changes in temperature and rainfall, species diversity, and migration patterns, influence and ultimately contribute to variability within and between foraging groups.

An examination of social change and the factors related to the introduction of herding in southern Africa can also be framed within the Culture Transmission paradigm. Culture Transmission is predicated on the idea that "similarity in behavior and artifacts may be caused by the exchange of information using a nongenetic mechanism” and focuses on the actions and decisions of individuals in cultural evolution (Eerkins and Lipo 2007: 240). Individuals receive information from various sources and make decisions about how to act on this information - to ignore it, accept it, modify it, or experiment with it (Eerkins and Lipo 2007).

This aspect of Culture Transmission theory can be extended to ideas about the inception and adoption of herding in southern Africa. Evidence attests to the mobility and extensiveness of trade networks operating among southern African groups throughout the Holocene. For example, marine artifacts have been found at inland sites like Apollo II, Boomplass, and Sehongkang that are up to 200km from the coast (Mitchell 2001). Sharing between and among mobile foraging groups and their neighbours was not restricted to material goods: ideas, conceptual frameworks, and worldviews were also shared. Operating within this framework, it is plausible to suggest that southern African foragers acquired, or at least encountered, ideas and materials associated with the herding lifestyle on various occasions, but it was not until certain factors, such as environmental change, population pressures and resource availability, were at play that particular foraging groups made the decision to adopt some of these outside ideas and materials, and integrate them into their 
situation. Aspects of the aforementioned theoretical paradigms will be embodied in this research to propose that subsistence variability can be ascertained by examining various aspects of the human skeleton. Spatial and temporal variability in skeletal morphology will be examined in an effort to contribute to the debate over the mechanisms responsible for lifestyle change.

\subsection{The Physical Geography of the Cape Region of South Africa}

The area under study lies at the southernmost part of the African continent, extending from $34^{\circ}$ to $32^{\circ}$ latitude, and $24^{\circ}$ to $28^{\circ}$ longitude. It is historically known as the Cape. The physical environment of the Cape region exhibits great physical diversity across a relatively small area. Moving from the west to the east and from the coast to the interior a number of different physiographic zones are encountered. Diversity of floral and faunal species reflects differences in rainfall and climate across this region. The Cape region includes the Western, Southern, and Eastern Capes, and spans the area from the coast up to and including the Cape Folded Mountains. Moving inland from the southern coast three distinct environmental zones are encountered that extend across the entire Cape region. The coastal foreland is interspersed with rocky outcrops and sandy beaches along its length. The coastal foreland is an area of high productivity which has experienced a very long history of human occupation, as evidenced by the numbers of sites that dot the coast (Deacon 1976). The coastal plain lies just inland from the coast and extends to the base of the Cape Folded Mountain belt. This area, of highly variable width, is transected by rivers and valleys that run into the sea. The coastal plain receives significant amounts of rainfall, but these amounts vary across the Cape region (Schapera 1930; Deacon 1976). 
The Cape Folded Mountain belt spans the entire coastal region from the extreme west to the east. However, the size and magnitude of this mountain range is variable throughout the region. In the South-western and Southern Capes the Cape Folded Mountains create a physical barrier between the coast and coastal plain and the inland grassland regions. The extreme elevation of this section of the mountains would have posed challenges for forager mobility and movement to inland locales.

The climate of the Cape region is similar to that found along the Mediterranean (Klein 1994). The amount of rainfall that the Western Cape receives is variable ranging from as little as $200 \mathrm{~mm}$ to as much as $2000 \mathrm{~mm}$ (Anonymous, 2007), and follows a seasonal pattern with the most rain falling during the winter months (Klein 1994; Shulze 1997). In contrast, the eastern parts of the Southern Cape receive rainfall throughout the year (Schapera 1930; Shulze 1997), but rain is predominant in spring and autumn (Deacon 1984). Mean annual temperature is uniform across the Western and Southern Capes with seasonal differences resulting in maximum (warm season) and minimum (cold season) temperatures that range from $26^{\circ} \mathrm{C}$ and $7^{\circ} \mathrm{C}$ in the west (Cape Town) to $25^{\circ} \mathrm{C}$ and $7^{\circ} \mathrm{C}$ in the south (Town of George) (Anonymous, 2007).

The Western Cape comprises the floral types of strandveld (vegetation of the sandy western coastal plain), coastal rhenosterbosveld, coastal fynbos, macchia and false macchia (Acocks 1988). Edible plant foods that were exploited by Holocene foragers consist primarily of geophytes (plant bulbs) and berries (Deacon 1993, 1984). The flora exhibits less seasonality in the Southern Cape than the Western Cape (Deacon 1984). The Southern Cape consists of a relatively small area of forest along the coast in the Knysna region and is comprised by the Knysna forest veld type (Acocks 1988.). Evidence from the Southern Cape 
suggests that foraging of gathered foods occurred in the open heathland and grassland areas in the vegetation mosaic (Deacon 1984: 258). The floral and faunal species diversity and biomass is lower in the forest than in the fynbos or savanna areas.

The Eastern Cape region presents unique climatic and environmental factors that set it apart from the Southern and Western Cape regions. In the Eastern Cape, the Cape Folded Mountain belt does not create as great a physical barrier between coastal and inland areas. Mobility between the coastal forelands and the inland locales is easier, and there is more continuity of floral and faunal communities between the coast and inland regions. Rainfall does not follow a seasonal pattern, but rather falls throughout the year making food resources available year round. As compared to Western and Southern Capes, the Eastern Cape comprises an intricate latticework of vegetative biomes. Most of the Eastern Cape is comprised of savannah, being present in two distinct forms: arid savanna and moist savanna (Acocks 1988; Cowling and Hilton-Taylor 1997). In the western portion of the Eastern Cape region (the focus of this study) a number of phytogeographical areas also converge: Namakaroo, grasslands, small fynbos satellites, and large areas of forest (Cowling and HiltonTaylor 1997; Midgely et al. 1997; O’Connor and Berdenkamp 1997; Rutherford 1997) (Figure 2.2). There is more continuity of floral and faunal communities between the coast and inland regions in the Eastern Cape because of the termination of the Cape Folded Mountain belt in the western part of this region. The area of greatest interest in this research is almost exclusively a year-round rainfall zone which results in high yearly accumulations ranging between 200 to 2000mm (Schapera 1930; Deacon 1984; Schulze 1997; Anonymous 2007). Mean annual maximum and minimum temperatures are consistent with the Western 
and Southern Capes, ranging between $25^{\circ} \mathrm{C}$ and $9^{\circ} \mathrm{C}$ (the city of Port Elizabeth) (Anonymous, 2007).

Figure 2.2 - Biomes of southern Africa. Eastern Cape study area indicated. (Map adapted from Rutherford 1997).

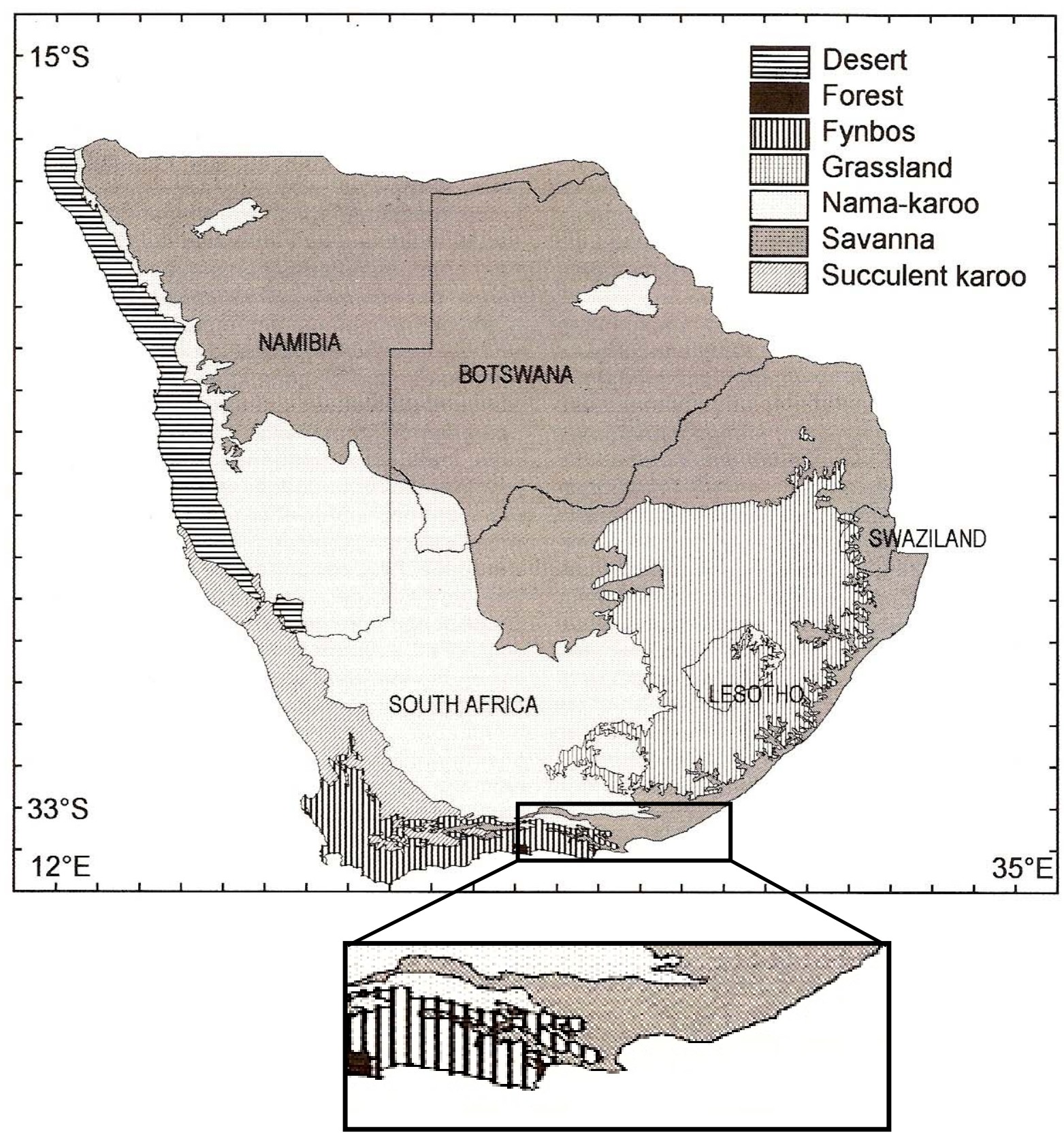


The lack of seasonality, year round rainfall, continuity of vegetation, and the congregation of five distinct vegetation types make this area the most productive and plentiful of the Cape Region, with very rich plant species diversity (Cowling et al 1997:462). Even the sand dunes along the coast promote the growth of plants that are adapted to dry costal systems, providing suitable forage for grazers (Lubke et al. 1997). Analyses of floral remains from Eastern Cape sites indicate that foragers exploited a particular set of plant remains that were widely available throughout the year and the region (Deacon 1984). Edible plant foods, mainly geophytes, are plentiful and diverse in this region (Deacon 1976; Churchill and Morris 1998). The Eastern Cape is rich in plant species, but the fauna consists mainly of small browsers because of the low grass element in the vegetation (Deacon 1976).

In general, the climate and vegetation that existed in the Cape during the Holocene was similar to present conditions (Rosen et al. 1999), yet short term climatic variations have been recorded. Climatic and environmental conditions in southern Africa began to change somewhat beginning around $4500 \mathrm{BP}$, initiating a period of significant decreases in temperature and increases in rainfall. This widespread change is thought by some to have been the impetus for the introduction of animal domestication in this area (Sadr 2004; Sealy 2006). Cunningham and Davis (1997) suggest that domestication was so delayed in this part of the world because wild plant and animal food resources were so abundant, diverse and readily available so that human dietary requirements were met. However, rapid and dramatic climatic changes along with other social modifications in the mid Holocene, as discussed subsequently in greater detail, required that inhabitants diversify their subsistence behaviors in response to these changing conditions. As the findings of the current research and that of others (e.g., Hall 1990; Binneman 1996; Sealy and Pfeiffer 2000; Pfeiffer and Sealy 2006) 
attest, during the period between approximately 4000 to $2000 \mathrm{BP}$ it appears that this environment was no longer able to support the nutritional requirements of the existing population, requiring the exploitation of new and varied food sources. Temperature and rainfall changes promoted modifications to the local floral habitats, in turn affecting the faunal species that could be supported. In many parts of the southeastern region, moister and cooler conditions favoured the growth of shrubs over grasslands, resulting in a subsequent increase in smaller game in faunal assemblages (Mitchell 2002). Climatic changes concomitant with increasing population pressures created a situation that encouraged innovation, experimentation, and social and subsistence diversification as a means to cope with these stressors and ensure continued prosperity in this region.

\subsection{Who were the Holocene Foragers of the South African Cape?}

Although foraging groups living across the Cape region of South Africa display some variation in their behavior and lifestyle as a consequence of the different environments in which they lived, these groups are thought to have been genetically and culturally homogeneous prior to the arrival of herders around 2000 BP (Deacon and Deacon 1999; Mitchell 2002a).

The lithic technology of Later Stone Age Holocene foragers of the South African Cape can be classified as a microlithic scraper/backed element industry that was produced by foraging groups across the southern region of South Africa (Deacon 1984; Klein 1994). The LSA, which includes all archaeological assemblages dating within the last 20000 years, has been subdivided into the Robberg (22000 to 12000 ya), Oakhurst (12000 to 8000 ya), and Wilton (8000 BP until 2000 ya) lithic complexes (Deacon and Deacon 1999). The bow and 
arrow was the primary tool used by hunters, while the stone weighted digging stick was employed by gatherers to extract underground plant foods (Deacon 1984).

Although there are regional differences in the types of animals hunted and the kinds of foods gathered for subsistence, some generalizations can be made about the subsistence behaviours exhibited by Holocene foragers living in South Africa. Similarities in lithic technology, social organization and subsistence behaviours throughout southern Africa suggest that a shared foraging way of life and extensive trade network existed from the Zambezi River to the South African Cape (Mitchell 2002a). During the early Holocene archaeological and faunal evidence suggests that foraging groups relied upon larger migratory bovids for subsistence. However, around 8000 BP the focus on terrestrial species shifted to smaller, solitary browsers who maintained a constant territory and did not follow a seasonal migration (Deacon 1984). Although Holocene foragers hunted, it is thought that most of the diet was composed of gathered foods, primarily underground corms and bulbs (Deacon 1993, 1976). Marine foods appear to have been an important component of Holocene forager subsistence, increasing in importance after 6000BP (Binneman 1996; Mitchell 2002a). The remains of marine fauna, particularly shellfish, are present at both inland and coastal sites across the southern region. Faunal assemblages indicate that marine resources were important to Later Stone Age foragers (Buchanan et al. 1984; Klein 1994).

The size of southern South African foraging groups may have varied throughout the year. All or most Holocene foragers are believed to have practiced a seasonal migration, but some argue that the climate of the Western Cape required that groups migrate to different areas to obtain seasonally available resources (Deacon and Deacon 1999). Such seasonal migration could also involve alteration of band size, involving aggregation during the winter 
when resources were plentiful and dispersal of smaller groups to exploit less abundant summer resources. The availability of food and water also dictated when forager groups would aggregate and disperse. The predominance of winter rainfall in the Western Cape, as compared to year round rainfall in the Southern and Eastern Capes may have required Western Cape foragers to travel to other areas in the winter to obtain resources (Deacon 1984). As will be subsequently discussed, population densities appear to have increased and groups may have become less mobile towards the end of the prehistoric period.

Considerable archaeological research has been conducted in the Eastern Cape region (Binneman 1996a, 1996b, 1997, 1998, 1999, 2000, 2001, 2004/05; Binneman and Hall 1993; Hall 1990, 2000; Hall and Binneman 1997). It forms the basis of our understanding of the prehistory of this region, yet given its size and topographic diversity this vast area remains somewhat understudied, relative to the Southern and Western regions. Research suggests that Eastern Cape foraging populations may not have been as isolated as Southern and Western Cape foraging populations. Unfortunately, little work has been conducted with regard to seasonal transhumance in the Eastern Cape (Mitchell 2002a). Judith Sealy is currently conducting research on stable isotope data collected from Holocene foragers from the Eastern Cape for purposes of dietary reconstruction. This forthcoming information will help to provide a more complete picture of Eastern Cape subsistence and may also provide some indication of the extent of the mobility of these foraging groups.

\subsection{Evidence for Intensification of the South African Foraging Lifestyle}

Regional variability in foraging subsistence practices and the seasonal mobility that was practiced by foragers has been observed across the southern South African coastal region throughout the Holocene. In some parts of the Western and Southern Capes, faunal and lithic 
evidence suggests that forager subsistence followed a seasonal round in which inland floral and faunal resources were exploited in the summer while during the winter months food procurement focused on coastal and marine resources (Parkington 1972; Buchanan et al. 1984; Deacon and Deacon 1999; Parkington 2001). In contrast, stable isotope data collected from South-western Cape Holocene skeletons suggests that groups did not migrate between the coast and inland sites at different times throughout the year (Sealy 1986, 2002; Sealy and van der Merwe 1986).

While the scope of forager mobility may have been regionally variable, foraging peoples made spiritual and symbolic connections with their environment and would tend to return to familiar areas year after year (Hall 2000). Stable isotope evidence corroborates this claim and indicates that Holocene foragers were buried in the home ranges that they occupied in life (Sealy 1986, 2006; Sealy and van der Merwe 1986). Archaeological data (Hall, 2000; Mitchell 2002a) and dietary stable isotope research (Sealy 1986; Sealy and van der Merwe 1986) indicate that towards the end of the Holocene foraging peoples became more territorial, reducing their mobility and establishing closer ties to their home ranges. Isotopic evidence from prehistoric sheep in the south-western Cape suggests that herders did not follow a seasonal pattern of transhumance during the Holocene (Balasse et al. 2002), adding further evidence for increasing territoriality of mid-to-late Holocene groups.

Around 3500 BP a number of major behavioural and social changes appear to have taken place in the foraging lifestyle (Table 2.1). Few controlled excavations of sites with good stratigraphic sequences (Nelson Bay Cave) spanning the entire Holocene period have been undertaken along the southern coast. A shift in forager subsistence behaviour around 3300BP was first postulated by Inskeep based on the Nelson Bay Cave sequence. These 
transitions are postulated on a broader regional scale. They signify a differentiation of lifeways and an increase in variability among foraging groups (Mitchell 2002a, b). Initially, these changes were thought to be associated with the arrival of herders (Deacon 1984). However, recent research suggests that environmental change brought about by climatic fluctuations in the region, particularly an increase in annual rainfall and reduction in temperature which altered vegetation and the dependent faunal species, coupled with the stress of a growing human population contributed to corresponding modifications to the diet, behaviours and social structure of foraging groups (Cunningham and Davis 1997; Deacon and Deacon 1999).

Table 2.1 Changes in forager behaviour between $3500 \mathrm{BP}$ and 2000BP (Deacon 1984; Deacon and Deacon 1999; Hall 2000; Mitchell 2002a)

\begin{tabular}{|l|}
\hline Tools became informal and were made primarily of quartzite \\
\hline Increased exploitation of freshwater mussels \\
\hline Population density increased \\
\hline Territories become smaller \\
\hline Groups became less mobile \\
\hline Increased reliance on local foods and resources \\
\hline
\end{tabular}

This shift towards resource intensification and increasing forager territoriality can be attributed to a culmination of a number of factors. Around $8000 \mathrm{BP}$ a cooling and drying trend across southern Africa stimulated a widespread evacuation of interior regions of South Africa by foraging groups in favour of more habitable and productive localities to the south (Deacon 1976, 1984). Over time the results of this influx of foragers coupled with increasing population size among the indigenous inhabitants of the southern coastal region began to impact local resource availability. Hall (1990) and others (Inskeep 1986; Jerardino Wiesenborn 1996) have observed that around 4500 BP foraging groups throughout the Cape region began to initiate a shift in their dietary resources from more terrestrial foods towards a 
greater focus on riverine and marine foodstuffs. Another short lived, yet dramatic climatic oscillation characterized by general decrease in temperature and increase in rainfall with corresponding decrease in oceanic surface temperatures (Cohen et al. 1992) and advancing sea levels (Jerardino 1995) occurred between 3200 and 2400 BP (Lee-Thorp et al. 2001; Mayewski et al. 2004; Lewis 2005) across the southern region. This may have also contributed to the move toward intensification and been partly responsible for the reorientation of resource exploitation towards aquatic resources, as foraging groups undertook a widespread regional domestic occupation shift from rock shelters to large open air sites (Mitchell 2002a).

Other subsistence changes, such as an increased focus on marine resources, are viewed as indicators of complexity by Binford (2001). These factors, as well as food storage, processing and surplus are defined by Woodburn (1982) as hallmarks of a delayed return economy, indicating a move away from the traditional egalitarian forager way of life. Sealy (2006) identified differences in carbon and nitrogen stable isotope values of two neighbouring hunter-gatherer groups who cohabited the southern Cape Coast between 4500 BP and 2000 BP. She suggests that this indicates the establishment of territories, differential access to certain marine resources, and a reduction in mobility. The sudden appearance of massive, homogeneous deposits of marine shell, termed 'megamiddens', in the absence of evidence of terrestrial or marine animal remains at sites along the western and southern coasts further indicates a shift of subsistence behaviours (Jerardino 1995, 1997). A shift in forager subsistence behaviour towards food production and storage is evidenced by the presence of charcoal throughout shell midden layers coupled with the absence of domestic artifacts suggesting that mussels were being processed at the coast and the dried meat 
transported and consumed inland (Henshilwood et al. 1994). Stable isotope dietary evidence and food remains from archaeological sites indicate that in this region between 3500 and 2000 BP marine foods became more important to foragers than at any other time during the Holocene (Sealy and van der Merwe 1988; Sealy et al. 1992; Jerardino and Yates, 1996). Storage pits associated with Holocene forager occupations have also been identified at a number of sites in the Cape region around this time (Deacon 1984; Hall 1990; Binneman 2004/05). Evidence of reduced forager mobility and increasing territoriality extends beyond subsistence behaviours. While the continuity in lithic traditions and technology shared by foragers across this region was maintained during this period, Binneman (2004/05) has noted the increased variability in stone tool technologies of contemporaneous Holocene huntergatherer groups from a small geographic area along the south-eastern coast of South Africa. He interprets this as evidence of reduced mobility, and the establishment of social and physical boundaries.

The skeletal remains of foragers who occupied the Cape region between 3500 and 2000 BP provide corroborating evidence for a period of stress that adds further support to the widespread nature of this intensification event. Sealy, Pfeiffer, and others who have conducted archaeological and osteobiographic research on western and southern Cape Holocene populations in South Africa, have found evidence of stress possibly associated with social instability. Sealy and Pfeiffer (2000) suggest that the small body size of Holocene foragers may have served as an adaptation to an environment where the topography is in sharp relief and food resources were at times unpredictable. Pfeiffer and Sealy (2006) suggest that increased variability in stature and body size corresponds with the period of intensification. During the period of intensification and diversification of foraging 
behaviours between 3300 BP and 2000BP the body size of some Southern Cape foragers declined still further. However, just prior to 2000 BP, adult stature became less variable, returning to earlier dimensions, which are comparable to the stature of historic Khoesan people. Recent work by Pfeiffer and colleagues (Pfeiffer et al. 1999; Pfeiffer and van der Merwe 2004; Pfeiffer in press) has identified evidence of interpersonal violence from this time period.

Much of the archaeological research on forager intensification has been conducted in the Western and Southern Capes, regions where seasonal climate and ruggedness of the Cape Fold Mountains could affect the availability of resources, possibly leading to partitioning of regions and resources in times of scarcity and stress. In an environment like the Eastern Cape where physical geography places fewer limits on resource availability and there is less geographical isolation, one would expect that cultural boundary formation may be less common. Yet, differentiation in stone tool technology, food procurement strategies, and burial style and location (Hall and Binneman 1987; Hall 1990; Binneman 1996; Hall 2000; Binneman 2005) indicates that some degree of social differentiation and territoriality, comparable to that observed in the other two Cape regions, was developing among Eastern Cape hunter-gatherers between 4500 and 2000 BP.

This shift towards increased forager territoriality and the move toward a delayed return economy did not persist throughout the Late Holocene. Binneman (1998) suggests, based on floral and faunal evidence, that foraging groups again became smaller and more mobile during the last 2000 years, because of pressure exerted by the existence of pastoral populations in the region. It has been suggested that the changes to social and economic aspects of the foraging lifestyle that are indicative of a delayed-return economy, including 
reduced mobility, increased connections with the land, emerging territoriality, and food storage, may have laid the groundwork for the adoption of herding by indigenous southern African foragers around 2000 BP (Sadr 2004; Sealy 2006). Smith (1998) has asserted that foragers would not be able to make the transition to a way of life that was dependent on storage and conservation of resources, because of their egalitarian social organization and worldview. However, if conditions were in place that promoted or demanded that foragers move to a more delayed-return approach, that transition could have allowed foragers to gain the skills and experiences necessary to adopt a more intensive delayed return economy, like herding. It is possible that some groups made the transition to herding because social and population pressures were making it increasingly difficult to maintain a hunting and gathering way of life. It is possible to speculate that the differential adoption of herding may have alleviated enough stress on populations living along the southern coast that it was then possible for some groups to return to a more traditional foraging economy after $2000 \mathrm{BP}$, as observed by Binneman (1998).

\subsection{Subsistence Changes and Population Movement in sub-Saharan Africa}

Economic, social, and ecological changes related to the intensification of foraging behaviours that occurred around 3500 BP may have played some role in the adoption of herding in southernmost Africa. The potential effects of subsistence, mobility, climate and environmental changes in other parts of sub-Saharan Africa during the Holocene must also be considered. On the African continent, root crop farming is believed to have originated in West Africa around the sixth millennium BP, while agricultural practices involving cereal cultivation and herding of domestic stock are thought to have developed in North-East Africa around the eighth millennium BP (Vansina 1996). All forms of agriculture appear to have 
slowly spread toward the south-east, becoming firmly established during the succeeding millennia north of the Zambezi River (Vansina 1996). The south-east spread of the agropastoral complex beyond the Zambezi River (also known as the Bantu Expansion) did not take place until the beginning of the second millennium BP (Vansina 1996; Phillipson 2005; Huffman 2006). A detailed review of the complexities of the Bantu expansion and the debates surrounding this event are beyond the limits of this study. However it is pertinent to briefly outline the basic tenets of this expansion, since this event had implications for social and economic developments farther south.

The factors responsible for the monumental and rapid southward expansion of agropastoral populations are not fully understood. The spread and timing of food production is variable across different parts of Africa (Marshall and Hildebrand 2002). Population pressures, climate and environmental changes have been cited as possible factors spurring the initial expansion of food producers out of the northern savannah region (Marean 1992; Holl 1998; Phillipson 2005). The aridification of the Sahara during the seventh to sixth millennia BP has been suggested as the stimulus for the initial dispersal of food producers, with pastoralism gradually spreading to East Africa around $4000 \mathrm{BP}$ and later to southern Africa at around 2000 BP (Gifford-Gonzalez 1998, 2000). The impact of aridification on subsistence is reinforced by the identification of isotopic evidence of dietary change among groups inhabiting the Libyan Sahara between 4000 to 2000 BP (Tafuri and Di Lernia 2006). The pattern and speed of the transition to food production is similar in East and southern Africa, with pastoralism preceding agriculture as the initial food producing economy (Marshall 1990, 2000). 
A second major wave of agropastoral expansion out of East Africa took place around 2000 BP reaching South Africa around 1200 BP (Huffman 2006). Analysis of languages and dialects in the Bantu language family supports the two stream Bantu dispersal model (Louw and Finlayson 1990; Finlayson 2006). Increases in summer rainfall, which were needed for the successful cultivation of many African cultivars, may have been a major factor of the initial south-east spread of farming (Prins 1996). Bantu-speaking agropastoralists are thought to have settled in attractive regions of northern Botswana and Zimbabwe sometime during the early first millennium $\mathrm{AD}$, before making their final push southward along the east coast of South Africa. These genetically and culturally distinct Iron Age ${ }^{1}$ mixed agriculturalists finally settled in the eastern fringes of the Eastern Cape area around $1300 \mathrm{BP}$, an area previously inhabited only by foragers (Silberbauer 1979; Binneman 1996; Boonzaier et al. 1996; Lewis 2002).

One of the major limiting factors to the southern spread of agropastoralist groups with domestic stock were the tsetse fly barriers, as well as other diseases that could be transmitted to herds via contact with indigenous wild species (Gifford-Gonzalez 1998, 2000). According to A. Smith (2005) a tsetse free corridor that corresponds with the hypothesized route of sheep into southern Africa existed during the time of the agricultural expansion.

The Kalahari region, like the South African Cape region, has been occupied continuously for the past 80,000 years by foraging groups, as evidenced by LSA and MSA deposits at sites like the White Paintings Rock Shelter in the Tsodilo Hills, Botswana and Apollo II in Namibia (Robbins et al. 1994, 2000). Studies indicate that the interactions between these migrant food producers and the indigenous inhabitants of Botswana and Zimbabwe were variable (Mazel 1989; Morris 1993; Vansina 1995; Denbow 1999). Some

\footnotetext{
${ }^{1}$ Archaeological evidence of these food producing agricultural groups is normally labeled 'Iron Age (IA)'
} 
believe that foragers entered into client relationships with the agriculturalists (Denbow and Wilmsen 1986) while others believe that contact between these groups was restricted to trade (Soloway and Lee 1990). The presence, yet relative scarcity, of artefacts associated with Iron Age groups in archaeological forager contexts in the Kalahari region attests to the existence of long distance trade with distant groups (Lee 1993) as does the presence of sheep remains and pottery in conjunction with a LSA lithic assemblage dated to $2150 \mathrm{BP}$ at the Bambata Cave in Zimbabwe (Walker 1983). Research conducted by Morris and others suggests that Later Stone Age inhabitants of the Zambezi Valley region (Zambia, Zimbabwe and Malawi) had a closer relationship with migrant farming groups than previously thought. The results of craniometric analyses suggest that LSA inhabitants of the Zambezi Valley are morphologically distinct from their hunter-gatherer contemporaries in South Africa and instead exhibit closer affinity to Bantu-speaking groups (Morris 2002, 2003; Morris and Ribot 2006).

\subsection{Evidence for the Emergence of Herding in Southern Africa}

Sheep are first seen from around 2000 BP in the Cape region of South Africa (cf. Klein 1986; Deacon and Deacon 1999). After that date, traditional foraging was supplemented by new food resources among some groups inhabiting the Cape coastal region. There are no wild ancestors to domestic sheep in sub-Saharan Africa. Domestic sheep that were herded prehistorically originated in the Near East from the wild Asiatic mouflon, Ovis orientalis (Klein 1986). Sheep had been a domestic fixture among African horticultural and pastoral populations further north long before their introduction to southern Africa. The first evidence of small domestic stock on the African continent can be traced to the seventh millennium BP in North Africa (Clutton-Brock 1993; Marshall 2000) and later spread to 
northern parts of East Africa by the fifth millennium BP (Robertshaw 1989, 1993; GiffordGonzalez 1998, 2000). A diversified form of pastoralism was first established in East Africa whereby it appears that hunting and gathering continued to form the major subsistence focus with domestic stock constituting a very small dietary contribution (Marean 1992; GiffordGonzalez 2000). It is not until ca. 3000 BP that specialized pastoralism focusing exclusively on domestic food production developed in East Africa (Marshall 1990, 2000; Marshall and Hildebrand 2002). The emergence of pastoralism follows a similar trajectory in southern Africa beginning around $2000 \mathrm{BP}$.

Some disagreement initially existed among researchers regarding the ability to distinguish between the remains of closely related ovicaprid species like sheep and goats, prompting many early studies to tentatively identify ovicaprid faunal remains as sheep/goat (Schweitzer 1974), but criteria for identifying sheep are now well established (Voight 1986). The ability to distinguish sheep from goats has bearing on the debate surrounding the timing of the introduction of herding. Goats are believed to be a more recent introduction to southern Africa.

While ethnographic accounts document historic Khoesan groups herding both sheep and goats, sheep were the first ruminant species to be introduced to southern Africa and remained the preferred ovicaprid species of southern African forager, herders and farmers (Clutton-Brock 1996, Voigt 1986). The presence of sheep in prehistoric contexts abounds in the archaeological record. Sheep of the fat-tailed variety are depicted in rock paintings from Zimbabwe to the Cape (Manhire and Parkington 1986). Goats are believed to be a later introduction to southern Africa, sometime around $1500 \mathrm{BP}$, and were not as valued as is 
evidenced by their scant representation in Iron Age faunal samples and their exclusion from rock art (Voigt 1986).

Considering that sheep (and all ovicaprid species) are not indigenous to Africa, their emergence in southern Africa had to have occurred via an introduction of some sort. The mechanisms and path surrounding the movement of sheep from their African home in east Africa to northern Botswana is generally accepted (cf. Klein 1986). Rather, the focus of the debate surrounding the emergence of sheep in southernmost Africa concerns the mechanisms that were responsible for this introduction - brought south by migrating groups or traded into the south via trade networks. While herder occupations have been found at a number of sites throughout South Africa, archaeological evidence for the presence of sheep around 2000 BP is limited to a few sites in the Cape Coast (Sealy and Yates 1994; Henshilwood 1996; Binneman 2004/2005) and the Northern Cape (Webley 1992, Vogel et al. 1997). Most early research operated under the assumption that sheep were brought to the Cape by migrating herding groups. Two possibilities regarding the identity of these herders were put forth: either they represented Khoe herders who originated in northern Botswana in the region of the Zambezi River, or they constituted Iron Age agropastoralists associated with an early Bantu migration. Recently, the addition of new approaches has stimulated a reassessment of the existing evidence prompting challenges and amendments to these alternatives.

\subsection{Evidence for Migration vs. Diffusion}

\subsubsection{Migration}

The migration theory focuses on the idea that non-indigenous groups moved into South Africa from northern regions, bringing sheep and the herding lifestyle with them. Skeletal remains that are identified from herder contexts are thus thought to represent these 
foreign migrants. Some postulate that the herders who migrated into South Africa were derived from aboriginal hunters living in southern Africa, probably northern Botswana, who adopted aspects of a new socio-economic system from elsewhere through contact with Early Iron Age groups when they settled in the area (cf. Boonzaier et al. 1996; Deacon and Deacon 1999). Alternatively, others believe that the herders who arrived in South Africa constituted small culturally and genetically distinct Early Iron Age groups who migrated from their temporary settlements north of the Zambezi River in advance of their Bantu-speaking kin (Smith 1986.).

Linguistic, social and physical anthropological data were utilized by various researchers to develop theories about the migration of herding groups and to propose routes that the migrant herders would have taken en route to southern Africa. Stow (1905) was the first to offer a route of dispersal. Based on oral traditions Stow suggested that herders began their migration near Tanzania, moving south to Zimbabwe, west to Namibia through northern Botswana and then south to the Cape and finally east to the Eastern Cape. Cooke’s (1965) path followed a similar route as Stow's, but it originates farther south in Zimbabwe and is based on environmental zones and vegetation along with art associated with sheep. However, Dewar's (2007) study of the Atlantic coastal region of the Northern Cape did not uncover archaeological evidence of early herder occupation, suggesting that route of entry for sheep into southern Africa may not have been along the Atlantic coast as Stow and Cook suggest. Elphick (1977) constructed a different, multifaceted route, citing ethnohistoric and linguistic information. He proposed an initial migration that saw herders move directly south from Botswana along the eastern edge of the Karoo to the coast. From there groups moved along Indian Ocean coast in both a westward and eastward direction. Elphick postulates that 
the existence of herding groups in the Northern Cape and Namibia are the result of a second, later wave of herders moving west along the Orange River to the Atlantic coast and then spreading north into Namibia and south into the Northern Cape.

The distribution of sites with domestic stock and firm radiocarbon dates offer some support for Elphick's proposed route. The Salumano site in Zambia dating to the around $2300 \mathrm{BP}$ is the earliest found containing sheep remains (Voigt 1986). The Bambata site in Zimbabwe contains sheep remains that date to around $2150 \mathrm{BP}$, while the other two sites with sheep remains with BC dates are located along the southern coast of South Africa at Die Kelders and Boomplas, both dating to the early second millennium BP (Voigt 1986). The period surrounding 2000 BP saw extensive population movement and subsistence change in other regions of Central, West and East Africa (Voigt 1986; Morris and Ribot 2006), making it seem plausible that movement and social change could have created a domino effect initiating change in other areas of Africa that eventually trickled down to South Africa.

The timing of the introduction of herding in South Africa and the migrations and subsistence changes in other parts of Africa coincided with the alleviation of Tsetse fly belts. This change allowed domestic stock to move southward into zones that were previously uninhabitable. Jordan (1986 as cited in A. Smith 2005) suggests that even in prehistoric times a tsetse free corridor existed, which happens to coincide with one of the proposed routes of sheep migration from the Great Lakes region of Kenya through Tanzania, and Zambia into Zimbabwe. However, the fact that small stock are less affected by tropical disease than cattle has been cited as yet another factor for the spread of sheep into southern Africa in advance of cattle (A. Smith 2005). 
Differences in the style of rock art north and south of the Zambezi River have been cited as support for the migration of herders. In South Africa, rock art associated with San foragers depicts detailed animals through the use of fine brushwork, yet north of Zambezi River rock paintings are more geometric and have been linked with the central African geometric art tradition (B. Smith 2006).

The distribution of rock art sites depicting sheep also supports the migration of herders and corresponds with the migration route proposed by Elphick (1977). Most paintings of sheep are found along a corridor that stretches from Zimbabwe down through the eastern portion of South Africa to the southern Coast and in the southern portion of the Western Cape (Manhire et al. 1986). Sites with fat-tailed sheep paintings have been found scattered throughout the southern coastal region as far east as Grahamstown. Manhire and colleagues (1986) assert that the number of sites with possible sheep paintings is underappreciated because the Eastern Cape remains largely unexplored. Interestingly, in the Western, Southern and Eastern Capes sheep paintings are found in the mountain regions in areas that were not suitable for grazing (Hall 1986). This information, in conjunction with the fact that sheep are painted in the same style as other rock art depicting wild animals, has been taken to indicate that foragers were the authors of these paintings (Manhire et al. 1986). They were documenting contact with herders living in the same general region. In the Eastern Cape, rock paintings of sheep are located in the rock shelters in the coastal plateau and coastal foreland, while cattle paintings are found only in the inland areas of the escarpment (Hall 1986). Hall (1986) suggests that the distribution of paintings indicates the progressive displacement of foragers from coastal to inland regions, first by herders and later by agropastoralists. 


\subsubsection{Diffusion}

The diffusion argument for the introduction of sheep herding to southern South Africa is centered on the idea that sheep were obtained by existing indigenous foragers via trade networks. It is speculated that when the conditions may have necessitated the adoption of sheep herding, some foragers opted to focus their subsistence on sheep herding while others maintained their traditional foraging lifestyle. Substantial evidence has been presented to support this possibility. Sadr (2003), one of the main proponents of this argument, likens the situation to the Neolithic transition in the Middle East and Europe, where indigenous foraging groups abandoned foraging in favor of farming. Evidence suggesting an indigenous development of herding has also been uncovered in Namibia (Kinahan 2001). Intertwined in this suggestion is the argument that it is possible for a group to alter their social and ideological systems and transition into a new way of life. Continuing research into the intensification of South African LSA foraging groups around 3500 BP is being cited as evidence to support this theory. Indications of a shift not only in behaviour but also ideology with the observed reduction in mobility, increased territoriality and evidence of food storage (Binneman 2004/2005; Sealy 2006) suggest that the conditions were suitable for such a subsistence shift to occur. Continuity in stone tool assemblages coupled with difficulties in distinguishing forager from herder occupations (Parsons 2006) add further support for this hypothesis. It has been argued that ovicaprid species are the easiest to care for of all domestic stock because they are not as susceptible to diseases, can survive even in marginal environments, and because of their small size are easy to trade (Smith 2005). These factors would make them attractive to foragers in difficulty. 
On the other hand, Smith $(1986,1998,2006)$ asserts that, because of their egalitarian social organization and worldview, foragers would not be able to make the transition to a way of life that was dependent on storage and conservation of resources. While this idea has some merit, it does not consider the conservation principals that promote the sustainability of wild resources inherent in forager worldview and cosmology. Surplus and storage are minimal or nonexistent in direct-return foraging societies, but the ways that foragers interact with their environment promote the sustainability of wild resources. Many foraging groups practice controlled burning of vegetation to promote plant growth and attract valued game, and collect and hunt only the amount of food resources that are needed (Deacon 1976, Deacon 1993). All southern African LSA foraging groups shared certain cultural, social and technological commonalities, yet regional variability has been observed that stems from differences in climate, environment, species diversity and resource availability. The range of forager variability, flexibility, and adaptability, the intensification of forager subsistence behaviours across the Cape region between 3500 and 2000 BP, coupled with the ability of contemporary southern African foragers, like the Ju/'hoansi, to incorporate aspects of other subsistence strategies into their socio-economic system, all speak to the ability of foragers to modify their subsistence strategies when the situation demands. Therefore, if conditions were in place that promoted or demanded that foragers move to a more delayed-return approach, that transition could have allowed foragers to gain the skills and experiences necessary to adopt a more intensive delayed return economy, like herding.

\subsection{Distinguishing Foragers from Herders}

Attempts have been made to distinguish foragers and herders on different grounds, including archaeological settlement and material culture, linguistics, rock art, and genetics 
and biology. While a number of differences and similarities have been proposed for the analysis of forager and pastoralist occupation sites, burial sites, dietary remains and rock art, it has not proven possible to confidently distinguish herders from hunters archaeologically, historically and ethnographically. Difficulties in differentiating between these two subsistence approaches arise in part from the inherent socio-economic and cultural similarities of herding and foraging groups, as well as the history of close contact between groups.

\subsubsection{Occupation Sites}

Archaeological research at two nearby sites in the Western Cape, Kasteelburg and Witklip, has enabled researchers to tentatively identify patterns that can be used to distinguish forager from herder sites. Criteria are based on differences in artifact frequency and composition. Smith and colleagues (1991) designated Witklip as a forager site based on the presence of fine microlithic stone tools, small diameter ostrich egg shell (OES) beads, very few potsherds, and a faunal assemblage comprised mainly of undomesticated local mammals. Conversely, Kasteelburg was identified as a herder site on the basis of the presence of pottery, a faunal assemblage composed largely of sheep and seal bones, large diameter OES beads, and coarsely made stone tools (Smith et al. 1991; Boonzaier et al. 1996; Smith et al. 2000).

Even with the creation of herder and forager cultural packages, difficulty exists in identifying and distinguishing herder occupations because of the fluid nature of foraging and herding lifestyles. The extent to which sheep fulfilled the dietary protein needs of herders was variable. Sheep were slaughtered for their meat, while sheep milk and blood served as sustainable food sources for southern African herders. Herding groups may have continued 
to rely heavily on hunting for subsistence, and groups that lost their stock may have reverted to foraging temporarily until stock could be acquired (Klein 1986). Milk, a stable source of protein, was only seasonally available. In historic times, domestic stock was slaughtered only on special occasions (Elphick 1977). The inability to devise solid herder archaeological signatures also arises from the mobile nature of these groups who needed to move frequently in search of suitable pasturage. Difficulties distinguishing hunter and herder sites are not restricted to the Cape coastal region, but have also been experienced by researchers working on sites in the Northern Cape (Parsons 2006).

Ceramics have been thought to have accompanied the introduction of sheep, thus forming an identifiable herder 'package', and potentially supporting the argument that the advent of herding in southern Africa was an offshoot of Early Iron Age migrations. However, as Sadr and others suggest, the distribution, chronology and style of the earliest southern African ceramics suggests an alternative inception (Sadr 2006).

Foragers, while also very mobile, are more visible in the archaeological record owing to their recurrent occupation of rock shelters and cave sites, and their longer and more extensive use of the landscape. Ethnohistoric accounts document that some forager groups acquired stock through theft or were employed as herdsmen by herding groups (Elphick 1977; Boonzaier et al. 1996). In such cases, the percentage of sheep bones could be expected to be minimal when compared to wild faunal assemblages. Klein (1986) suggests that sites with low frequencies of sheep remains and pottery could be interpreted to be forager sites, while those with higher frequencies of these items be attributed to herders. However, the different dietary roles that sheep fulfilled would have resulted in different archaeological signatures. If sheep were butchered and consumed then a significant quantity of skeletal 
remains could be expected, while if sheep were relied upon mainly for their blood and milk few remains would be present. Voigt $(1984,1986)$ points out that even at Iron Age sites solidly associated with agricultural occupation the remains of domestic stock are often less numerous than wild remains because agriculturalists relied heavily on foraging at various times. Therefore, it is only possible to make tentative forager-herder assignments based on the presence and quantity of sheep bones. Acknowledging these practices, it is even more difficult to distinguish between hunter and herder sites, since sites with both herder and hunter signatures could reflect occupation by hunters who acquired stock, or seasonal occupations by herders who were practicing foraging behaviors.

Various Eastern Cape sites that date to the last 2000 years have been identified as having been occupied by foraging groups. They contain certain artifacts that are associated with herders, but only in small quantities (Binneman 1998, 2000). Therefore, it is obvious that simple distinctions between herders and foragers cannot be safely made based on the presence of pottery, sheep remains, or the size of OES beads alone.

\subsubsection{Burial Sites}

Burial commonalities have been observed across South Africa during the Holocene, yet burial variability in both time and space exists. Along the southern coast, from the Western to the Eastern Cape, foragers buried their dead in caves and rock shelters (Hall and Binneman 1987; Jerardino et al. 2000; Sealy et al 2000), while in the Northern Cape and western Orange Free State foragers were buried almost exclusively in open site burials (Inskeep 1986). Spatial and temporal differences in burial fill, shape, and inclusions have been documented both archaeologically and ethnographically. Grave good composition and frequency is inconsistent, even among burials that are associated in time and space. Early 
Holocene burials across the southern coast display a strong association with red ochre, but are lacking in grave goods (Webley 1997). During the mid-Holocene Western and Eastern Cape burials exhibit not only the most grave goods of any other time period, but also the most elaborate range of goods. While some individuals were buried with a very elaborate range of goods during this period, such as ostrich egg shell water containers and beads, shell ornaments, painted and bored stones, grindstones, tortoise bowls, others lack any grave inclusions (Webley 1997). However, the magnitude of this temporal and spatial pattern may be over-estimated. Considering that the interior region appears to have been largely uninhabited during this time, there are few burials with which to make comparisons (Inskeep 1986). Late Holocene burials in the Cape region are characterized by fewer grave goods than earlier burials. Some Eastern Cape juvenile burials (Klasies River Mouth Burial 5 and Melkhoutboom) exhibit elaborate inclusions while most southern Cape burials are stark during this time (Webley 1997). Although differences in burials have been observed throughout time and space, all ages and both sexes of southern African foragers appear to have been treated similarly in death. Burial similarities between prehistoric foragers and modern San groups reinforce the continuity between all southern African foraging groups through time. Even though the burials of prehistoric foragers were more elaborate than those of the San, prehistoric and modern southern African foragers bury their dead in a fetal position, include a gravestone in the burial, and afford males, females and children similar burial treatment (Webley 1997).

Attempts have been made to distinguish foragers from herders based on burial characteristics. However, as with attempts made to distinguish between herder and forager occupation sites, the spatial and temporal variability in prehistoric southern African burial 
practices makes establishing a solid dichotomy difficult. Inskeep (1986) attempted to differentiate between forager and herders using a number of burial characteristics. A list of differences in herder and forager burial practices is provided in Table 2.2. In the absence of a cairn burial feature or an individual placed in a seated position, attribution of an individual as a herder based on burial characteristics must be considered tentative.

Table 2.2. LSA burial practices: forager and herder differences (Inskeep 1986)

\begin{tabular}{|c|c|c|}
\hline & Foragers & Herders \\
\hline Grave stones & Flat on top of body & $\begin{array}{l}2 \text { layers: one top of body \& } \\
\text { large stones in grave fill }\end{array}$ \\
\hline Body Position & Flexed on side & Flexed sitting \\
\hline Grave goods & Various inclusions & Not typically included \\
\hline Shape & Round oval & Square \\
\hline Depth & Shallow & Deep with side niche \\
\hline Surface covering & Flat stone & Mound, 'cairn' \\
\hline Location & $\begin{array}{l}\text { Coastal caves, rock shelters; } \\
\text { inland rock shelters, caves, } \\
\text { middens }\end{array}$ & $\begin{array}{l}\text { Open grassland; river } \\
\text { tributaries }\end{array}$ \\
\hline Ochre & On grave stone & Absent \\
\hline
\end{tabular}

\subsection{Relationships between Southern African Foragers and Herders: Existing Beliefs and Evidence}

\subsubsection{Linguistic Evidence}

Historic Khoe and San groups both spoke click languages not shared by other groups, although clicks are also used by some agricultural groups who acquired these elements through contact (Cavalli-Sforza et al 1994). Historic Khoe and San dialects included distinctive words and structures. Westphal (1963) suggests that historic Khoe spoke a dialect related to the Central Bush family of languages, as spoken by foragers living in northern Botswana and western Zimbabwe (cited in Klein 1986). In terms of the linguistic diversity, or lack thereof, between Khoe and San, it is not clear whether these differences 
indicate a recent evolution of language brought about by group contact or actual differences that existed in the distant past.

\subsubsection{Skeletal Evidence}

Although the human skeleton has not been extensively utilized as an avenue to investigate this issue, the human cranium has been the focus of much of the research. Early research into the differences between living and skeletal forager and herder samples, or 'Bushmen' and 'Hottentots'2 as they were then referred, was typological in nature and predicated on the belief that these two groups shared a common ancestral origin but that they were physically, culturally, spiritually and socially different (Morris 1987). Study samples were biased from the start, since they were selected from known-in-life individuals or were lacking archaeological context or any other information that could be used to attribute them to either forager or herder groups. These studies had the objective of isolating certain sets of features based on racial typologies that the researchers themselves had determined to reflect pure forager or pastoralist forms. Living individuals or skeletons needed only to possess one character in order to be assigned to one of the two categories, and any specimens exhibiting variability were classified as hybrid rather than attempting to reconcile the nature of the variation (Morris 1986).

The champions of the first craniological studies, Shrubsall (1898, 1907, 1911, 1922), Broom (1923) and Keen (1947), believed that Khoe and San were different, so their results fulfilled that philosophy (Morris 1986). Research conducted in the 1960s through the early 1980s by De Villiers (1968), Rightmire (1970, 1972, 1978), and Hausman (1980) attempted to overcome the typological biases in study samples by selecting historic individuals that

\footnotetext{
2 These terms were commonly used at the time that the research was conducted, but are no longer used as they are considered derogatory by some.
} 
were 'known-in-life' as Khoe or San. While this effort was commendable, consideration was not given to the fact that these known specimens were collected and classified using the same racial typologies that the researchers were trying to move beyond, nor was the possibility of significant genetic admixture from other groups considered (Morris 1992c). Temporal and contextual information about study samples was often lacking, making herder and forager designations very suspect.

It was not until the latter part of the twentieth century with the work of Morris (1984, 1992b) that research became more objective, relying on radiocarbon dating, additional lines of evidence to identify forager and herder specimens, and employing more sophisticated statistical techniques. Recently, Stynder (2006) undertook a craniometric study that explored the range of cranial variation through time from the Pleistocene to the Holocene. Stynder was unable to identify significant differences within his LSA sample that would support a hypothesis of significant genetic change.

\subsubsection{Genetic Evidence}

Genetic research has indicated that many genes are common to all African groups (Nurse and Jenkins 1985; Cavalli-Sforza 1994; Stoneking 2006), yet this information alone cannot be used to ascertain population affinity. Genetic data, both mtDNA and Y chromosome, support a recent African origin for all extant human groups (cf. Vigilant et al. 1991; Ingman et al. 2000; Underhill et al. 2000; Stoneking 2006). More recently, genetic research on click speaking Hadzabe hunters from Eastern Africa and Ju/'hoansi foragers from southern Africa, suggests that the separation of these two groups occurred around the time of the origin of Homo sapiens sapiens sometime upwards of 70,000 years ago (Knight et al. 2003). 
Although genetic evidence has established that San foragers and Khoekhoe herders share the same ancient southern African heritage (Chen et al. 2000), this information cannot provide insights into the history of mobility or the mechanisms that may have been responsible for the introduction of herding. The results of a genetic distance study conducted by Cavalli-Sforza highlight the difficulties involved in trying to establish genetic relationships between groups closely related in space and time. Cavalli-Sforza (1994) found that when genetic distances are calculated only for Bantu-speaking and Khoesan groups, the Khoe appear more closely related to all Bantu-speaking groups than to the San, yet the San are more closely related to the Khoe than to any of the Bantu-speaking groups. The presence of certain Gm alleles in Khoe and Bantu-speaking populations that are not present in San populations suggests recent genetic admixture between the two groups (Nurse et al 1985), yet this information does not provide insight into the relationship between ancestral herders and foragers since all three groups share a number of Gm alleles. Early research by Jenkins (1970) found that Xhosa groups, those being southern African Bantu-speaking farming groups who inhabited the most south-eastern edge of the agriculture supporting summer rainfall zone, exhibit the greatest proportion of San admixture of all the southern Bantu-speaking groups. These examples illustrate how geographical proximity or population movement produce genetic similarity, but genetic information alone is not sufficient to inform us about the behaviors that brought about population admixture.

\subsection{Hypotheses}

The skeletal morphology of the LSA inhabitants of the Eastern Cape will be examined in order to test hypotheses pertaining to the biological relationship between herders and foragers, and the mode through which herding was introduced to the Eastern Cape 
region. The null hypothesis will be that these skeletal remains belong to one gene pool, thus making it possible to look internally to make within-group and between-individual comparisons. If any changes in skeletal morphology are observed, the point in time during which the change occurred will be key in establishing the factors responsible. Differences in adult skeletal morphology, as indicated by differences pertaining especially to cranial and dental size and shape, limb size and proportions, and discrete (non-metric) cranial traits, will be viewed as evidence of genetic differences within the skeletal sample. If the null hypothesis is not correct, there should be differences between temporally defined subgroups, including differences in the size and/or shape of the cranium, dentition and postcranial skeleton, indicating two populations, with herders exhibiting larger body size and taller stature.

1. The LSA inhabitants of the Eastern Cape constitute a single biological population. Herding was introduced by means of diffusion. Significant skeletal change is not expected at around 2000 BP. Biological continuity will be demonstrated by the absence of significant changes in skeletal form; changes in body size may occur but not at $2000 \mathrm{BP}$. This is the null hypothesis $\left(\mathrm{H}_{0}\right)$.

2. There is evidence of biological discontinuity among the LSA inhabitants of the Eastern Cape. Herding was introduced by means of migration (by a genetically distinct group). Biological discontinuity will be demonstrated by the presence of significant changes in skeletal form and body size beginning at around 2000BP. This is $\mathrm{H}_{1}$. 


\section{Chapter 3 - Materials and Methods}

\subsection{Materials}

Adult skeletal remains of Holocene foragers that have been recovered from various sites within the Eastern Cape Province of South Africa will form the basis for this research (see map - Appendix1). The Eastern Cape sample is comprised mainly of skeletons curated by the Albany Museum (ALB) at Rhodes University, Grahamstown, Eastern Cape Province, South Africa, with smaller numbers selected from the Dart Human Skeletal Collection at the Department of Anatomy (A), Witwatersrand University, the skeletal collections of the National Museum of Bloemfontein (NMB) housed at the Florisbad Research Station, skeletal collections from the Department of Anatomy and Cell Biology (UCT) at the University of Cape Town, and the Pre-Colonial Archaeology Collections at the Iziko Museum of Cape Town (Table 3.1). A complete list of the Eastern Cape specimens is provided in Appendix 2.

Table 3.1 Distribution of skeletal remains by Institution

\begin{tabular}{lc|ccc}
\hline Collection & Total N & Complete skeletons & Crania only & Postcrania only \\
\hline A & 7 & 4 & 2 & 0 \\
ALB & 54 & 34 & 4 & 18 \\
NMB & 3 & 0 & 3 & 0 \\
UCT & 4 & 0 & 4 & 0 \\
Iziko & 5 & 2 & 3 & 0 \\
\hline & $\mathbf{7 3}$ & $\mathbf{4 0}$ & $\mathbf{1 6}$ & $\mathbf{1 8}$ \\
\hline
\end{tabular}

The nature of the question being explored in this research demands temporal control. It is therefore essential that the skeletal remains selected for inclusion in this study could be attributed to the Holocene period either through radiocarbon dating, or through direct archaeological association with the radiocarbon dated remains. The Eastern Cape sample dates between 8260 and $240 \mathrm{BP}$ (uncalibrated). The temporal distribution of the sample is almost equally split with forty individuals predating $2000 \mathrm{BP}$ and thirty-three postdating the 
2000 BP benchmark (Appendix 2). Most radiocarbon dates that have been obtained for these selected Eastern Cape skeletons are unpublished and all are uncalibrated. I have received permission from J.C. Sealy and S. Pfeiffer to use the radiocarbon dates obtained for the ALB skeletal remains, and D. Stynder to use the radiocarbon dates for the A, UCT, NMB, and Iziko remains.

Radiocarbon dates are expressed as a mean and standard deviation, but because of fluctuations in the concentration of atmospheric ${ }^{14} \mathrm{C}$ they do not correspond directly with calendar years. In North America and Europe, tree ring data that track fluctuations in atmospheric carbon have been used to construct calibration curves that enable radiocarbon dates to be expressed as calendar years BC or AD. Although calibration curves have been developed for the southern hemisphere they have not been extensively used by those in southern Africa working on topics concerning the Holocene period (Mitchell 2002). Another problem encountered in the calibration of radiocarbon dates in South Africa, especially those generated from human bone collagen, concerns differences in the rate in which carbon from terrestrial and marine protein sources is combined with ${ }^{14} \mathrm{C}$ from the atmosphere and incorporated in human tissue (Sealy and Pfeiffer 2006). Because of this, mixed diets that include a marine protein component will produce older radiocarbon dates (Pfeiffer and Crowder 2004; Sealy and Pfeiffer 2006). Given the fact that the previous research on topics in southern African prehistory has been conducted using uncalibrated radiocarbon dates, it is appropriate for this research to operate in similar terms such that comparisons with published work can be made. When employed as a variable in this research, the rounded uncalibrated date (without standard deviation), is used as a single datum. 
The skeletal sample is comprised of seventy-three adult skeletons (females $n=42$, males $n=31$ ) from thirty-six localities in the Eastern Cape Province. Of these, 65 individuals have been radiocarbon dated. The remaining eight individuals from St. Francis Bay, Welgeluk, Spitzkop, Middlekop and Wilton sites can be considered contemporaneous with the dated individuals based on archaeological contextual information that links them to this time period. The individuals lacking radiocarbon dates originate from sites with multiple interments where controlled excavations were undertaken. When bone samples were collected for radiocarbon dating, the researchers (S. Pfeiffer and J. Sealy) decided that when individuals were associated in a burial context only one bone sample would be collected in order to minimize destruction (S. Pfeiffer personal communication). For the purpose of this analysis, those skeletons without dates were assigned the radiocarbon date of the individuals with which they were associated.

All of the available adult Eastern Cape skeletal remains that demonstrate temporal or contextual evidence sufficient to link the remains with a foraging or herding lifestyle were included in the sample in an effort to maximize sample size. The completeness of the skeletal remains curated by the various South African Institutions, as well as the archaeological contextual information, is variable. This is partly because of differences in the manner in which the skeletal remains were collected. Large numbers of the skeletal remains were the product of controlled excavations and have been curated with accompanying information about the archaeological context, manner and style of burial and cultural affiliation. Yet, many museum specimens are the product of chance collections or donations by individuals unaware of archaeological protocols. Over half of the specimens included are represented by complete or nearly complete skeletal elements, with smaller 
numbers represented only by cranial or postcranial remains (Table 3.1). Regardless of the variable range of information available from each skeleton, the skeletal sample is sufficient to explore the questions posed by this research.

\subsection{Methods}

The methodological approach of this research can be divided into two parts. First, a general assessment of the skeletal remains, in the form of an osteobiography of each skeleton, was conducted. Metric and discrete variables were then recorded and subsequently subjected to statistical manipulation.

\subsubsection{Data Collection}

Most skeletal data were collected with the aid of the Smithsonian Institution's Repatriation Osteology Laboratory (ROL) data-entry software. This software package was developed by Steven Ousley of the Office of Repatriation, Smithsonian Institution, National Museum of Natural History (NMNH) for use by the repatriation osteology laboratory to document their aboriginal skeletal collections that are to be repatriated. The ROL operates in a Microsoft Access database format and is based on the approach to the collection of skeletal data developed by Buikstra and Ubelaker (eds.) in Standards for Data Collection from Human Skeletal Remains (1994) (Ousley et al. 2005). Buikstra and Ubelaker devised their approach to the collection of skeletal data in response to the need to develop a standardized data collection protocol that would satisfy the needs of archaeologists, biological anthropologists, researchers, and descendent aboriginal groups involved with NAGPRA and the repatriation process for standardized, comprehensive and directly comparable data. This approach to data collection has been adopted by many skeletal biologists conducting research outside the realm of NAGPRA and has become a standard for data collection in the field. 
The ROL program is organized into categories of skeletal data including: Bone Inventory, Age and Sex, Pathology, Postcranial Metrics, Craniometrics, Cranial Nonmetrics, Dental Inventory/Development/Pathology, and Dental Morphology. The researcher can create a form for each skeletal individual and information can be entered in each of the aforementioned categories. The data are stored and organized by category of skeletal information, not by individual or specimen. When data collection is complete the data can be opened in a Microsoft Access database, from which the information can be exported to other programs for further statistical manipulation.

Most of the skeletal information relating to the skeletal inventory, skeletal indicators of age and sex, information regarding the condition and health of the dentition, cranial and postcranial measurements, cranial discrete traits, and skeletal pathology was collected using the ROL program. In some cases the data collection capabilities or categories of skeletal data available in the ROL data entry software were insufficient for the purposes of this research. The ROL software is limited to the more common cranial discrete traits and does not provide an interface suitable for the recording of information on skeletal indicators of activity or all expressions of skeletal pathology. This additional information was recorded manually in Microsoft Word or Excel and then later combined with the ROL data.

\subsubsection{Skeletal Assessment}

Any skeletal analysis project dealing with archaeologically derived skeletal remains must address questions relating to the validity of using standards developed from modern, known identity skeletal samples to categorize past populations for whom the growth and ageing trajectories cannot be determined. Most methods of skeletal identification that are used in osteological analyses have been developed on skeletal reference samples derived 
from documented North American cadaveral skeletal collections that are comprised mainly of individuals of European and African descent. As such, these methods, and their inherent assumptions, may not be ideally applicable to populations that are not represented in these skeletal reference samples. In addition, it is recognized that the most accurate estimation of skeletal identity is possible through the assessment of complete, well preserved skeletal remains, yet archaeologically derived skeletal remains are often fragmentary and poorly preserved. By employing multiple methods, assessing all available skeletal elements, and gaining an appreciation of the baseline skeletal variation of the sample under study, it is argued that a sufficient assessment of skeletal identity can be achieved.

\subsubsection{Age and Sex}

Sex was assessed by examining various morphological features of the cranial and postcranial skeleton known to exhibit size and shape differences between males and females. A number of morphological techniques based on the cranium (Buikstra and Ubelaker 1994) and pelvis (Phenice 1969; Buikstra and Ubelaker 1994) were employed to estimate sex for the Eastern Cape sample. Prehistoric Khoesan hunter-gatherer populations are small bodied and exhibit a distinctive pattern of sexual dimorphism, including relatively low cranial sexual dimorphism and relatively pronounced pelvic sexual dimorphism, as compared to other human population groups. Considering that most of the morphological methods of sex estimation have been developed on larger bodied and differently dimorphic populations, mainly North American populations of European and African descent, published morphological sex indicators may yield an elevated number of female sex estimates if sex is assessed based on cranial remains alone. Thus the range of sexually dimorphic features present in the study population was assessed in the context of pelvicly-determined sex, and 
was applied to attribute sex when the pelves were unavailable. The overall appearance of the skeleton with respect to size and rugosity was included in the final sex estimate.

Sex was initially assessed and, for the sake of consistency, was verified during a subsequent research trip one year later. Sex estimates were later corroborated with other researchers who had previously studied the same Eastern Cape skeletal remains (ALB: S. Pfeiffer, H. Kurki, and D. Stynder; A, Iziko, NMB, and UCT: D. Stynder). The sex estimates made during this research are consistent with those of other researchers for over ninety percent of the sample. Where disagreement existed, the sex assessments were reconsidered, but the assessments made by the current researcher were maintained as this researcher was most familiar with the range of variation exhibited during the time period and region in question.

Methods that focus on age-related changes to the pelvis, cranium, sternal rib ends, and dentition were used to estimate age. On the pelvis, morphological changes of the pubic symphysis (Suchey and Katz 1986; Brooks and Suchey 1990) and auricular surface (Lovejoy et al. 1985) were examined. Işcan and colleagues' method $(1984,1985)$ was used to assess age based on degenerative changes of the sternal rib end. Methods involving the closure of the cranial sutures (Meindl and Lovejoy 1985) and the obliteration of the palatal sutures (Mann et al. 1991) were also employed. The extent of dental wear was also assessed (Smith 1984). The overall condition of the skeleton, with respect to both age related degeneration and bone formation, typically associated with osteoarthritis, at the joint surfaces of the elbow, shoulder, knee, hip and vertebral column were considered in the final age estimate. For the purposes of this analysis, the various age indicators were used to place each individual in a general age category, rather than assign an individually unique age range estimate. 
Although the focus of this research is on the skeletal remains of adult individuals, adolescents (ca. 15-20 years) were included if their skeletal remains were sufficiently developed such that the appropriate metric and morphological data could be collected. Most of the methods that were employed to assess biological distance - cranial and dental measurements, and cranial discrete traits - can be assessed from the skeletal remains of adolescent individuals. In order for adolescents to be included in the osteometric component of this research, the epiphyses of the long bones had to be fused, with the exception of the medial clavicle epiphysis. Four criteria were employed in order to classify an individual as adult: fusion of long bone epiphyses, closure of the spheno-occipital (basilar) suture, fusion of the medial clavicle epiphysis, and eruption of the third molars. The development of the long bones and the teeth were recorded using the ROL program following the age standards presented in Buikstra and Ubelaker (1994).

\subsubsection{Health, Activity and Diet}

General information about the life history of an individual, including the health, diet, and physical activity or occupation can be reflected in the morphology of the human skeleton (cf. Larson 1997). All skeletal elements were examined for indications of the individual's relevant skeletal and dental morphology. Skeletal evidence of illness and disease, habitual activity, and trauma were recorded according to the Buikstra and Ubelaker (1994) protocol. Habitual patterns of activity were assessed by examining muscle insertion sites, as well as the shape and rugosity of the long bone shafts. This information was used to support the metric and morphological sex assessments.

The ROL program that follows the protocol outlined in Buikstra and Ubelaker (1994) was also employed to record information about the condition of the dentition. Dental wear 
was assessed following the scheme developed by Smith (1984) where each tooth is assigned a wear score between one and eight. The number and location of teeth lost antemortem was recorded. The condition of the dentition with respect to caries, alveolar abscesses and periodontal disease was recorded for the value that this information could add to the age estimate.

\subsubsection{Metric Data}

The metric component of this research attempts to describe the size and shape of various aspects of the skeleton through the recording of quantitative variables that are specific to the cranium, dentition and postcranial skeleton. The morphological component, which focuses on the cranium, concerns the recording of various discrete, or discontinuous, variables that are believed to reflect inheritance. The entire suite of metric and morphological variables could not be assessed for each skeleton in the sample due to differences in the degree of preservation and the condition of the skeletal remains. Variability exists in both the size of the sample and the number of variables that could be assessed per individual for each metric and morphological category of analysis.

\subsubsection{Craniometric Methods}

The craniometric variables employed in this study follow those outlined in Buikstra and Ubelaker (1994), based on the work of WW Howells (1973) and Martin (1957). A total of 47 craniometric variables were assessed and are listed in Appendix 3. Measurements of paired cranial elements were recorded on the left side; if the left side was damaged the right side was substituted. All cranial measurements were recorded to the nearest millimetre with the aid of sliding, spreading callipers, and co-ordinate callipers. Measurements were 
recorded in the ROL program and later exported to Excel to be formatted for statistical manipulation.

\subsection{Comparative Craniometric Data}

Comparative data available from other African samples are available for sub-Saharan African regions including Malawi, East Africa, West-Central Africa, West Africa, and Southern Africa (Ribot 2002; Morris and Ribot 2006) and for protohistoric herders and farmers from the Orange River region of South Africa (Morris 1992). Information is also available for selected craniometric variables from southern African Bantu-speaking ${ }^{1}$ samples (De Villiers 1967, Rightmire 1970). Ribot's (2002) Southern African sample comprises data from the same Bantu-speaking groups studied by De Villiers and Rightmire, so for the sake of consistency only Ribot's Southern African Bantu-speaking data will be compared.

\subsubsection{Odontometric Methods}

Two odontometric methods were used in this research. The well known maximum dental method developed by Moorrees and Reed (1954) measures each tooth at the widest points on the crown in the mesiodistal (MD) and buccolingual (BL) planes parallel to the occlusal surface. This method can only be applied to teeth that do not exhibit occlusal and interproximal wear beyond the point of greatest breadth. For purposes of standardization, only teeth that were assigned a wear score of less than six, according to Smith's (1984) dental wear scoring scheme, were included in this analysis. Measurements were recorded to the nearest 0.01 millimetre using fine tipped dial callipers.

The cervical method, recently developed by Hillson and colleagues (2005), overcomes the limitations imposed by wear by recording MD and BL measurements at the

\footnotetext{
${ }^{1}$ Bantu is a linguistic term that refers to agricultural groups who have their origins in Central Africa and who migrated southward and settled in what is currently known as South Africa around 1300 BP (Binneman 1996; Boonzaier 1996).
} 
cervix of the tooth. This method can be applied to teeth that exhibit significant wear so long as wear does not exceed beyond the cemento-enamel junction. Measurements of all suitable teeth were recorded to the nearest 0.01 millimetre using digital callipers specially designed by Hillson and FitzGerald for recording these specific measurement locations for in situ teeth. The maximum and cervical odontometric dimensions examined in this study are listed in Appendix 4.

Differences in the requirements of the two odontometric methods coupled with variability in the number of teeth present per individual and the suitability of each tooth for measurement result in differences in sample sizes between the two methods. Employing both methods ensured that the maximum number of individuals were included in the odontometric analysis. Both methods were applied to teeth that satisfied the requirements of the respective methods, while teeth that exhibited more advanced wear were assessed using only the cervical method. Teeth that did not meet the requirements of the respective methods, or that exhibited any pathological or taphonomic alteration of the enamel surface at the measurement locations were excluded. Right and left antimeres of all available maxillary and mandibular incisors, canines, premolars, and molars were measured.

\subsection{Comparative Odontometric Data}

Comparative odontometric data obtained from both skeletal and living populations exist for a range of sub-Saharan African groups. Maximum crown dimension data in the form of descriptive statistics (sample size, mean, standard deviation) of mesiodistal and buccolingual maximum diameters of maxillary and mandibular dentition are available for Western and Southern Cape LSA foragers (Pfeiffer in press), the early Griqua from South Africa (Keiser 1985), South African Bantu-speakers (Keiser et al. 1987), and the San 
(Drennan 1929). Because the cervical method of odontometric analysis has been developed only recently, comparative data are not available for Sub-Saharan populations.

\subsubsection{Postcranial Metric Methods}

Osteometric measurements were collected for all individuals with postcranial remains. The osteometric variables used in this study follow those listed in Buikstra and Ubelaker (1994) based on the measurement definitions of Moore-Jansen et al. (1994). A total of fifty-eight measurements were selected to characterize the size and shape of the upper limb (humerus, ulna, and radius), torso (clavicle, scapula, innominate, and sacrum), and lower limb (femur, tibia, fibula, and calcaneus, and first metatarsal). Size was assessed through measurement of bone lengths, breadths, heights, and circumferences, while shape was evaluated through anterior-posterior (A-P) and medial-lateral (M-L) shaft diameters. Osteometric variables could be collected for 57 individuals in the Eastern Cape sample. A complete list of the osteometric variables included in this research is provided in Appendix 5.

An effort was made to collect osteometric variables on both left and right postcranial elements. Measurements taken on left elements were preferred for use in the statistical analysis, but when the left side was unavailable measurements taken on the right were substituted. Measurement estimates were not made on fragmentary or incomplete remains unless the element could be adequately reconstructed in such a way that the integrity of the bone, and hence the measurement, was not compromised. Although reconstruction was usually possible, it was not always complete enough to allow for the collection of the entire suite of osteometric variables selected for each element. All postcranial measurements were recorded to the nearest millimetre using the appropriate measurement tool (digital sliding callipers, an osteometric board, or a measuring tape). 


\subsection{Comparative Postcranial Metric Data}

Limited osteometric data are available for a selection of sub-Saharan populations. Summary statistics for a selection of osteometric variables for East African, Central African (Pygmies), West African, and southern African San skeletal samples dating to the $19^{\text {th }}$ and $20^{\text {th }}$ centuries are accessible in Trenton Holliday's PhD dissertation (1995). Osteometric data collected by Lundy (1986) are also available for three South African Bantu-speaking skeletal samples: Cape Nguni, Natal Nguni, and Sotho. Comparative data are available for the following osteometric variables: femoral and humeral head diameter and epicondylar width, as well as humerus, radius, femur and tibia length.

\subsubsection{Statistical Analyses of Metric Data}

Similarities in the type of the metric data collected, as well as the nature of the research question being explored, dictates that a similar approach be taken to the statistical analysis of the craniometric, osteometric, and odontometric data. Unless otherwise noted, the following statistical discussion pertains to all three categories of metric data.

\subsection{Univariate Statistics}

Prior to analysis the skeletal sample was organized into four a priori groups based on sex and ${ }^{14} \mathrm{C}$ date:

1. Females: pre 2000 BP vs. post 2000 BP

2. Males: pre 2000 BP vs. post 2000 BP

3. Pre 2000 BP: males vs. females

4. Post 2000 BP: males vs. females

The metric dataset was scrutinized visually for outliers and anomalous data by plotting each variable independently by subgroup. To ensure that the metric datasets satisfied the 
assumptions of the selected univariate methods of analysis, individual metric variables were tested for normality using the Kolmogorov-Smirnov one sample test and for equality of variances using Levene's homogeneity of variance test. Summary univariate statistics (mean, standard deviation, and variance) were calculated for each craniometric, postcranial metric, and odontometric variable. Groups were then compared for each variable. Analysis of variance (ANOVA) was used to explore differences in the means for the various metric variables. An ANOVA will indicate which metric variables produce a significant result, but post-hoc tests are required to determine which of the a priori groups are responsible for the significant result. Since the sizes of the subgroups are unequal the GT2 and Games-Howell post-hoc tests were used to determine which subgroup means are significantly different at the 0.05 alpha level (Sokal and Rohlf 1995). All univariate tests of the Eastern Cape metric data were carried out with the aid of the SPSS 14.0 statistical computer package.

Comparative data are available only as summary statistics, which dictates the use of simple univariate statistics to assess possible differences between the study and comparative samples. SPSS 14.0 does not support the computation of t-tests using summary data (i.e. sample size, mean, and standard deviation), so the NCSS statistical software package was employed in the comparative analysis.

\subsection{Multivariate Statistics}

\subsection{Principal Components Analysis}

Once the univariate analysis identified basic patterns within the metric data, multivariate statistics were used to further explore these differences. Principal Components Analysis (PCA) was employed in an attempt to identify size and shape patterns in the metric data. PCA as a multivariate statistical method is very well suited to the composition of this 
skeletal sample, as well as the questions posed in this research, because it does not require the organization of a matrix into groups prior to analysis and is not adversely affected by small sample size, unlike other multivariate methods such as discriminant analysis. Rather, PCA considers individual skeletal entries for each metric variable in the calculation of the principal components, such that the PCA results can be explored across time (in this case), rather than as dichotomized groupings. Any possible morphological differences in the sample can be then be explored by plotting each principal component and examining potential patterns in the distribution of scores for each subgroup. In comparison to the ANOVA, which tests differences between individual variables, PCA seeks to reduce the number of variables considered while maximizing the amount of variation between groups of variables.

Before performing the PC analysis metric data must be standardized to remove the bias that differences in the magnitude of the various measurements may have on the results. The cranial, postcranial and dental datasets were separated into males and females and then the variables were transformed into z-scores. Transforming the raw data for the osteometric variables into z-scores standardizes the entire data set and relates it to a distribution with a mean of zero and a standard deviation of one (Bernard 1995; Madrigal 1995). Expressing the metric data as z-scores enables all variables specific to each region of the skeleton (crania, post crania, and dentition) to be directly compared, regardless of differences in magnitude of the variable (Hazard Monro 1997). Since the PCA was undertaken as an exploratory tool to investigate potential changes in skeletal morphology after $2000 \mathrm{BP}$ the decision was made to derive the PCA from a correlation matrix of standardized data rather than a covariance matrix of the original data. Using this approach all individuals in the sample can be incorporated 
into the analysis without the risk that sex-related size variation will overshadow potential morphological variation between the subsamples.

Commonly, in principal component analyses, the entire suite of metric variables is considered in calculating the principal components since the aim is to understand which aspects of the material under study exhibit the greatest variation. However, a PCA cannot be run on a data set with missing values, and the number of measurements available for each variable must be robust enough to allow for an adequate expression of the within-group variability in order to obtain an effective PCA result. The Eastern Cape sample does not include any skeletons that were complete enough to permit the collection of every metric variable. Therefore, the craniometric (total $\mathrm{N}=60, \operatorname{PCA} \mathrm{n}=49$ ), postcranial metric (total $\mathrm{N}=56$, PCA $\mathrm{n}=45$ ), and odontometric (total $\mathrm{N}=57$, PCA $\mathrm{n}=34$ ) analyses include different subsets of the total sample.

In order to obtain the most accurate representation of the variation expressed in the sample, a selection of particularly meaningful metric variables were identified. The selection of metric variables had to satisfy two conditions. First, the variables selected for the principal component analysis had to adequately reflect the range of morphology of each skeletal region under study. Second, variables had to be selected that would allow the maximum number of individuals to be included in each component of the metric analysis. Commonly, correlation coefficients are considered in the selection of metric variables for use in a PC analysis, whereby variables that are highly correlated are excluded from the analysis. Because the entire suite of metric variables was not available for each skeleton included the Eastern Cape sample a more intuitive, anatomical approach was used. The skeleton was divided into functional units specific to each of the three regions of the skeleton included in 
the metric analyses. The cranial variables were grouped to represent the three functional regions - vault, face, and mandible - and a set of variables that reflect the morphology, while allowing for the inclusion of the maximum number of individuals, was selected for each region. The postcranial metric variables were also separated into functional units - upper limb, trunk and lower limb - and a set of variables was chosen that enabled the greatest number of variables and the greatest number of individuals to be analysed. The odontometric variables were divided into anatomically functional units by tooth type - maxillary and mandibular anterior (incisors and canine) and posterior (premolars and molars) teeth - for the PCA. Care was taken to ensure that variables selected did not measure similar aspects of the skeleton, like, for example nasal height (nasion to nasospinale) and mid facial height (nasion to prosthion). The number of variables selected for each PC analysis differed between the functional units and was reduced from the original metric variable lists (Table 3.2).

Once the metric variable sets representing each functional unit were selected, the PC analysis was run on the individual z-score standardized entries for each group of variables. PCA will then reduce the multiple variables that have been selected to represent the functional units of the cranium, postcranial skeleton and dentition, into factors that represent the maximum range of size and shape variation reflected in the original variable set (Pimental 1992). The number of factors, or principal components, that should be retained is determined by consulting the eignvalues. The Kaiser criterion (1960) for the selection of factors, which stipulates that factors with an eignvalue greater than one should be retained for further analysis, was followed. Typically only the first and second PC scores are retained because the first PC score, and to a lesser degree the second PC score, is the most strongly associated with the original variables and accounts for the most variation (Pimental 1992). The PC 
Table 3.2 Metric variables used in the craniometric, odontometric and postcranial metric Principal Component Analyses. Refer to Appendices 3 - 6 for measurement definitions.

\begin{tabular}{|c|c|c|c|c|c|}
\hline \multicolumn{2}{|c|}{ Craniometric } & \multicolumn{2}{|c|}{ Odontometric } & \multicolumn{2}{|c|}{ Postcranial Metric } \\
\hline \multirow[t]{8}{*}{ Vault } & GOL & Maxillary Anterior & UI1CMD & Trunk & CLAVXL \\
\hline & BNL & & UI2CBL & & SACRANTB \\
\hline & $\mathrm{BBH}$ & & UCCBL & & ILIACBR \\
\hline & XCB & & UCCMD & & BIILIACBR \\
\hline & WFB & & UI2CMD & Upper Limb & HUMXL \\
\hline & ASB & Maxillary Posterior & UP1CBL & & HUMMIDXD \\
\hline & FRC & & UP1CMD & & HUMHEADD \\
\hline & PAC & & UP2CBL & & HUMEPBR \\
\hline \multirow[t]{9}{*}{ Face } & $\mathrm{NLH}$ & & UP2CMD & & RADXL \\
\hline & JUB & & UM1CBL & & RADHEADD \\
\hline & NLB & & UMICMD & & RADAPD \\
\hline & MAB & & UM2CBL & Lower Limb & FEMXL \\
\hline & $\mathrm{OBH}$ & & UM2CMD & & FEMMIDADL \\
\hline & OBB & Mandibular Anterior & LI1CMD & & FEMHHEDD \\
\hline & DKB & & LI2CBL & & FEMEPICB \\
\hline & FMB & & LI2CMD & & TIBXL \\
\hline & EKB & & LCCBL & & TIBPEPBR \\
\hline \multirow[t]{9}{*}{ Mandible } & GNI & & LCCMD & & TIBDEPBR \\
\hline & GOG & Mandibular Posterior & LP1CBL & & TIBAPNFD \\
\hline & WRB & & LP1CMD & & \\
\hline & $\mathrm{XRH}$ & & LP2CBL & & \\
\hline & MXML & & LP2CMD & & \\
\hline & MAN & & LM1CBL & & \\
\hline & & & LM1CMD & & \\
\hline & & & LM2CBL & & \\
\hline & & & LM2CMD & & \\
\hline
\end{tabular}

* Total metric variables examined in this study: craniometric $(n=56)$, odontometric $(n=32)$, postcranial metric $(n=57)$

scores are interpreted by examining the component loadings for each standardized variable.

A component, typically the first principal component, is interpreted to reflect size variation if all of the scores are positive, while components with scores of mixed signs, usually the remaining principal components, are interpreted to reflect shape variation (Jolicoeur and Mosimann 1960; Pimental 1992). The loadings represent the relationship between the component and the variable. Emphasis is placed on variables that have large scores for a particular principal component, as these variables make a greater contribution to the variance expressed by the principal component. Consequently, variables with small component scores 
are typically set aside as they have contributed minimally to the overall variance. Once the components with the greatest contribution have been selected, the loadings for each principal component are plotted, with loadings organized according to the a priori subgroups, to facilitate the interpretation of patterns relating to size (PC1) and shape (PC2) variation in the sample.

\subsection{Curve Estimation Regression}

Curve estimation regression was employed to further assist in the interpretation of the possible changes in the metric data through time. The Principal Component scores were regressed on the uncalibrated radiocarbon dates in an attempt to establish a relationship between these two variables. Prior to the regression analysis the metric datasets with PC scores were separated by sex and the curve estimation regression analysis was run on the male and female data separately. Curve estimation regression statistics were produced for both linear and quadratic models to determine which best suited the data and enhanced underlying patterns observed in the PCA results. Regression analyses that yielded large coefficients of determination $\left(\mathrm{r}^{2}\right)$ values coupled with statistically significant $(\mathrm{p}<0.05)$ values were interpreted to indicate the presence of a significant relationship between the dependent (PC score) and independent $\left({ }^{14} \mathrm{C}\right.$ date) variables. Size and/or shape patterns in the metric

data were further identified by grouping the skeletal sample by sex and ${ }^{14} \mathrm{C}$ date and plotting the regression outputs graphically.

\subsection{Population Genetic Analysis}

The mechanisms responsible for the advent of sheep herding in southern Africa can also be examined by undertaking a population genetic analysis of the genetic variability in the Eastern Cape skeletal sample throughout the Later Stone Age. Because changes in 
skeletal dimensions can reflect input from both environmental and genetic sources, it is important to try to pinpoint which microevolutionary forces may be responsible. A number of microevolutionary forces, such as gene flow, genetic drift and natural selection, can influence the genetic structure of a population. Gene flow and genetic drift are responsible for increasing genetic variation in a population. With this in mind, predictions about the resulting effects of migration and diffusion on genetic variation can be formulated. The introduction of new genes from a non-local source will increase the genetic variation of the local population, whereas the diffusion of an innovation or subsistence practice, like sheep herding, will have little effect on the genetic variance of the local population.

The traditional approach to reconstructing the genetic history of a particular population or group has been to calculate genetic distances between gene allele frequency data (cf. Cavalli-Sforza et al. 1994). Relethford and Blangero (1990) made the investigation of genetic variation more accessible to biological anthropologists by modifying the population genetic approach developed by Harpending and Ward (1982) to suit continuous quantitative data. Since its inception, the Relethford-Blangero model has been used in many biological anthropological investigations to examine changes in levels of gene flow as a means of exploring genetic relationships among and between populations (cf. Relethford et al. 1997; Steadman 1998, 2001; Powell and Neves 1999; Schillaci and Stojanowski 2005; Stojanowski and Schillaci 2006; Scherer 2007).

The extension of this population genetic approach to quantitative data does have some limitations. Population genetic analyses conducted on quantitative traits, rather than actual alleles, may not provide highly accurate information about the genetic structure of a population because the polygenic nature of these traits makes it difficult to minimize additive 
environmental influences. However, selecting quantitative traits that are known to be under stronger genetic control, and thus highly heritable, can minimize the environmental influences on the expression of phenotypic traits. Even though cranial and dental quantitative traits are under greater genetic control and are not as plastic as other areas of the skeleton (Sparks and Jantz 2002), cranial and dental traits vary to a certain degree in their heritability. A conservative assumption of the heritability value, like 0.55 , is believed to adequately account for this variation (Relethford 1994). However, Carson (2006) recently calculated estimates of heritability for a number of commonly used craniometric dimensions and found that heritability values may actually be lower for certain quantitative traits, some as low as 0.0 , indicating significant environmental influence. She warns against applying trait heritability values calculated for a particular population to other populations because environmental influences will be different in populations that are separated in time and space. Furthermore, while low trait heritability values may indicate low genetic influence, low heritabilities can also reflect an existing lack of genetic variation in a population resulting from natural selection, inbreeding and genetic drift (Stojanowski and Schillaci 2006: 59). For interest sake, Relethford-Blangero analyses were run using average heritability values of $0.4,0.55$, and 1.0 . Changing the average heritability value did not significantly affect the results, so the common average heritability value of 0.55 was used for reported results. The Relethford-Blangero analysis estimates levels of gene flow by comparing the observed and expected within-group heterogeneity across populations. The calculation of within-group heterogeneity is a complex mathematical procedure, the detailed discussion of which is beyond the limits of this study (but see Relethford and Blangero 1990). However, the analysis of genetic distance is methodologically accessible thanks to RMET 5.0, a 
quantitative genetic statistical program developed by J.H. Relethford that carries out the Relethford-Blangero analysis among other measures of genetic distance. RMET 5.0 is used to generate a scaled genetic relationship (R) matrix, from which observed and expected within-group heterogeneity are calculated.

As with any statistical analysis of quantitative data, there are a number of conditions must be satisfied before the quantitative dataset can be subjected to the Relethford-Blangero analysis. First, the dataset cannot include any missing values. Sixteen variables were selected that adequately characterize the morphology of the facial and vault region and are believed to demonstrate strong genetic control. This number of variables maximizes the number of individuals that could be included in the analysis (Table 3.3). A number of individuals were missing values for only one or two of the selected craniometric variables. For the sake of maximizing the size of the sample included in the population genetic analysis, the missing values were imputed from multiple regressions in the NCSS statistical program. This approach enabled the inclusion of an additional six individuals, increasing the total population genetic sample to thirty-five individuals.

Second, an estimate of relative effective population size must be provided for each of the populations included in the analysis in order to effectively estimate the relative levels of gene flow from foreign sources. Considering relative population size allows for the control of potential effects of genetic drift on small populations, thus ensuring the most accurate measure of gene flow (Relethford et al. 1997). Many studies have used the RelethfordBlangero analysis to explore genetic variation among settled populations where information about population size can be easily accessed from centralized burial contexts, habitation spaces, and site sizes. The mobile nature and small group size of the foraging and herding 
Table 3.3. Craniometric variables used in the Relethford-Blangero Analysis

\begin{tabular}{|c|c|c|c|}
\hline Measurement & Code & Source & Definition \\
\hline \multicolumn{4}{|l|}{ CRANIUM } \\
\hline Nasion Occipital Length & NOL & Howells (1973) & nasion (n) to opisthocranion (op) \\
\hline Cranial Base Length & BNL & Buikstra and Ubelaker (1994) & basion (ba) to nasion (n) \\
\hline Basion Bregma Height & $\mathrm{BBH}$ & Buikstra and Ubelaker (1994) & basion (ba) to bregma (b) \\
\hline Biauricular breadth & AUB & Buikstra and Ubelaker (1994) & auriculare (au) to auriculare (au) \\
\hline Biasterionic breadth & ASB & Howells (1973) & $\begin{array}{l}\text { point where temporal, occipital and } \\
\text { parietal meet }\end{array}$ \\
\hline Basion-prosthion length & BPL & Buikstra and Ubelaker (1994) & basion (ba) to prosthion (pr) \\
\hline Nasion-prosthion length & $\mathrm{NPH}$ & Howells (1973) & nasion $(\mathrm{n})$ to prosthion (pr) \\
\hline Nasal height & $\mathrm{NLH}$ & Buikstra and Ubelaker (1994) & nasion (n) to nasospinale (ns) \\
\hline Nasal breadth & NLB & Buikstra and Ubelaker (1994) & alare $(\mathrm{al})$ to alare $(\mathrm{al})$ \\
\hline Maxillo-Alveolar breadth & MAB & Buikstra and Ubelaker (1994) & $\begin{array}{l}\text { ectomolare }(\mathrm{ecm}) \text { to ectomolare } \\
(\mathrm{ecm})\end{array}$ \\
\hline Orbital Height & $\mathrm{OBH}$ & Buikstra and Ubelaker (1994) & $\begin{array}{l}\text { direct distance between superior and } \\
\text { inferior orbital margins }\end{array}$ \\
\hline Orbital Breadth & OBB & Buikstra and Ubelaker (1994) & dakryon (d) to ectoconchion (ec) \\
\hline Bifrontal Breadth & FMB & Howells (1973) & $\begin{array}{l}\text { frontomalare anterior (fma) to } \\
\text { frontomalare anterior (fma) }\end{array}$ \\
\hline Frontal Chord & FRC & Buikstra and Ubelaker (1994) & nasion $(n)$ to bregma (b) \\
\hline Parietal Chord & PAC & Buikstra and Ubelaker (1994) & bregma (b) to lambda (I) \\
\hline \multicolumn{4}{|l|}{ MANDIBLE } \\
\hline Chin Height & GNI & Buikstra and Ubelaker (1994) & infradentale (id) to gnathion (gn) \\
\hline
\end{tabular}

lifestyles makes the assessment of relative population size more complicated, and probably

less accurate. However, for the purposes of this analysis an accurate and precise estimate is not needed. Rather, a relative estimate of population size is sufficient. Archaeological and burial site data were used to arrive at a conservative estimate of relative population size for the pre- and post-2000 BP sub-groups. Population size is believed to have increased slightly in southern South Africa after 2000 BP with the advent of sheep herding. Thus, an estimate of a $25 \%$ population size increase was used for the post- $2000 \mathrm{BP}$ population. For the Relethford-Blangero analysis using RMET, the pre-2000 BP and post-2000 BP populations were assigned relative population estimate values of 1 and 1.25 , respectively.

Prior to subjecting the craniometric dataset to the Relethford-Blangero analysis, the data were separated by sex, standardized by transforming the measurements into z-scores and 
then recombined. The standardized data set was converted into a space delineated format and imported into the RMET program. When estimates of population size are provided, the unbiased genetic relationship (R) matrix is computed and is used to calculate the average distance to the regional centroid, as well as the observed and expected heterogeneity values across all variables for each population in order to minimize any potential influences of genetic drift (Relethford et al 1997). An estimate of gene flow for each subpopulation was obtained by calculating the residual heterogeneity. The residual heterogeneity, obtained by subtracting the expected within-group heterogeneity from the observed within-group heterogeneity, provides an estimate of the levels of gene flow from extraregional sources for each subpopulation. Both the magnitude and size of the residual value provide important details about the level of genetic variation. Positive residual heterogeneity values are generally interpreted to reflect greater than average gene flow from nonlocal sources, while to negative residual values indicate les than average gene flow. The greater the number from zero in either direction is a reflection of the magnitude of the genetic variation believed to result from gene flow. The significance of the residual values is obtained following the approach of Powell and Neves (1999). First, the standard error of the residual values is estimated by jackknifing the residual variance across the craniometric variables. The residual values are then divided by their estimated standard error, and this measure is compared to a $t$-distribution with $t-1$ degrees of freedom.

As a further means of exploring the range of genetic variation between the pre-2000 BP and post-2000 BP subpopulations, the standardized craniometric dataset used in the Relethford-Blangero analysis is subjected to PCA. The addition of genetic material from foreign sources will not only increase genetic variation within the indigenous group, but will 
also produce phenotypic changes in form. A MANOVA was then employed to determine if any of the PCs were significant as a means of assessing potential morphological variation across the Eastern Cape sample.

\subsubsection{Morphological Data}

\subsubsection{Cranial Discrete Data}

Forty-one cranial discrete traits, also known as non-metric, epigenetic or discontinuous traits, are explored in this study. Most of the cranial discrete traits were recorded using the ROL program, which incorporates the traits and manner of scoring presented by Buikstra and Ubelaker (1994). Seven additional cranial traits that are not included in the ROL program were added because they were deemed important to this research. A complete list of the discrete traits that are examined in this study and scoring procedures are provided in Appendix 6.

The manner in which each cranial discrete trait was assessed depended on the category of trait - hypostotic, hyperstotic, supernumerary bones, and features associated with vessels and nerves - and the range of expression of a trait. Most traits were assessed using a presence-absence approach; however, the complexity of some traits requires the use of additional categories to adequately describe the range of expression. Bilateral cranial discrete traits were recorded on both left and right elements when possible. If a trait could

not be observed due to missing or fragmentary skeletal elements, that trait was noted as being unobservable. Sex differences have been observed in the expression and frequency of some traits. Yet, the magnitude of the sex differences in trait expression and frequency has been found to be low (Molto 1983; Hauser and De Stefano 1989). Non-metric variables were assessed for all available crania regardless of the completeness or preservation of the 
remains. Individuals had to exhibit skeletal morphological sex indicators and display evidence that skeletal development was nearing completion in order to be included in the cranial discrete trait analysis. This approach allowed for the inclusion of fifty-nine individuals in the cranial non-metric analysis.

\subsection{Comparative Cranial Discrete Data}

Non-metric cranial data are available for a number of sub-Saharan populations. Summary statistics (sample size and mean) for a selection of cranial discrete traits are available for South African Bantu-speaking samples (De Villiers 1967, Rightmire 1972, 1976). The ability to make comparisons with cranial discrete frequency data from other South African skeletal collections is beneficial to this analysis.

\subsubsection{Statistical Analysis of Discrete Data}

Researchers have suggested using data from left and right antimeres for bilateral traits based on the assertion that a trait is under stronger genetic control if it is bilaterally expressed (Molto 1983, DeLaurier and Spence 2003). The side method was employed in this analysis whereby right and left scores for a particular bilateral trait are not pooled but treated individually. This approach to the analysis of discrete trait data is particularly important in prehistoric samples with fragmentary remains because it allows for the maximum number of individuals to be included in the analysis. Thus, the sample size (the number of right and left sides available) will vary for each discrete trait.

For ease of analysis, the discrete data are handled on a presence-absence basis.

Graded traits were converted to presence-absence following Molto (1983) and DeLaurier and Spence (2003). It was not possible to dichotomize all variables without losing important information. In these cases, the trait was expanded into multiple traits that would retain the 
detail of the graded observations. For example, metopic sutures were originally scored on as being absent, partial, or complete. In order to facilitate the analysis this variable was converted into two variables: 1. partial metopic suture, and 2. complete metopic suture. A total of 48 dichotomous discrete cranial traits were included in the analysis (Appendix 6).

Frequency statistics were calculated for each of the four subgroups. Since the goal of this study was to explore intra-sample variability commonly used statistical procedures that measure divergence between pairs of populations, such as C.A.B. Smith's Mean Measure of Divergence (MMD), would not have been appropriate. Such an approach would require the division of the sample into artificial sub-samples that would violate some of the central assumptions of the procedure. Instead, the Pearson chi square $\left(\chi^{2}\right)$ test is used to explore variation in discrete traits in the Eastern Cape sample. Although the chi square as a nonparametric test is not as robust a statistical procedure as the multivariate statistics it has the ability to identify basic relationships within the discrete data. For the $\chi^{2}$ results to be valid, the data must satisfy a number of assumptions: the data must be expressed as frequencies, sample size must be adequate (expected frequencies must be greater than five for each cell of a $2 \times 2$ table), the categories must be mutually exclusive, and the categorization of the sample must be meaningful (Munro 1997). In order to meet the sample size requirement the sample could not be divided into the four subgroups used in the metric analysis because dividing the sample along both sex and temporal lines as for some traits and categories (i.e. post-2000 BP males) would yield subsets that would be too small. Therefore, side differences in the expression of a particular discrete trait were explored separately first between males and females, and then between pre- and post-2000 BP individuals. The sample was pooled to explore possible sex and temporal differences in the cranial discrete traits. Significant $\chi^{2}$ 
values allow for the rejection of the null hypothesis that there is no difference between two groups for a given discrete variable. When the $\chi^{2}$ value is significant, the Phi value is used to determine the strength of the relationship between the pair of dichotomous variables (Munro 1997).

Comparisons between the Eastern Cape sample and the comparative samples are achieved using an on-line chi-square calculator (Ball and Connor-Linton 1996). Since frequency data are only available for the comparative samples and SPSS only allows for the calculation of chi-square using raw data, this alternative program was utilized. The discrete cranial sample is divided into subgroups by radiocarbon date (pre- and post-2000 BP) and relationships with the comparative samples are evaluated. 


\section{Chapter 4 - Results}

This chapter will summarize the results of the analysis of all the metric and discrete skeletal variables. Univariate statistics are used to explore size and multivariate statistics are utilized to investigate potential size and shape changes in the Eastern Cape skeletal sample. For purposes of analysis the Eastern Cape sample is divided into four sub-groups by date and sex: pre-2000 BP females, post-2000 BP females, pre-2000 BP males, and post-2000 BP. Summary statistics of the raw craniometric, maximum and cervical odontometric, and postcranial metric variables by sub-group are provided in Appendices 7 to 10. KolmogorovSmirnov tests confirmed that the distributions of the craniometric, odontometric and postcranial metric variables conform to a normal distribution (Appendices 11 to 14). The results of Levene's test for homogeneity of variances are provided separately for the craniometric, odontometric and postcranial metric variables in Appendices 15-18.

\subsection{Cranial Measurements}

\subsubsection{Univariate Analysis}

The craniometric sample $(\mathrm{N}=60)$ is the largest of all the Eastern Cape osteometric samples. When the four sub-groups are compared, the results of the one-way ANOVA do not provide evidence of significant differences in cranial size (Table 4.1). The post-hoc tests indicate that the greatest differences occur between males and females, reflecting the expected sexual dimorphism in the sample. Slightly more than half of the craniometric variables (24 of 47 variables) show pre-2000 BP females differing significantly from post2000 BP males. The list of significant variables indicates that size differences extend to all anatomical regions of the cranium. The pre-2000 BP females and males differ in only six variables that pertain to facial and mandibular breath and mastoid size. The differences 
between the post-2000 BP males and females are not marked yet they encapsulate the entire cranium, with means differing for 18 variables. As with the pre-2000 BP females, the post2000 BP females differ from the pre-2000 BP males on only a few craniometric variables (three) relating to facial projection, frontal length, and mandibular angle. Cranial size shows Table 4.1. Probability values for Craniometric Post-hoc1 ANOVA tests -variables that differ significantly between sub-groups $(p<0.05)$

\begin{tabular}{|c|c|c|c|c|c|c|}
\hline \multirow{2}{*}{$\begin{array}{c}\text { Craniometric } \\
\text { Variables }\end{array}$} & \multicolumn{3}{|c|}{ Pre 2000 BP Females } & \multicolumn{2}{|c|}{ Post 2000 BP Females } & \multirow{2}{*}{$\begin{array}{l}\text { Pre } 2000 \text { BP } \\
\text { Males } \\
\text { vs. } \\
\text { Post } 2000 \\
\text { BP Males }\end{array}$} \\
\hline & $\begin{array}{c}\text { vs. } \\
\text { Post } 2000 \text { BP } \\
\text { Males }\end{array}$ & $\begin{array}{c}\text { vs. } \\
\text { Post } 2000 \\
\text { BP Females }\end{array}$ & $\begin{array}{l}\text { vs. } \\
\text { Pre } 2000 \\
\text { BP Males }\end{array}$ & $\begin{array}{c}\text { vs. } \\
\text { Post } 2000 \\
\text { BP Males }\end{array}$ & $\begin{array}{l}\text { vs. } \\
\text { Pre } 2000 \\
\text { BP Males }\end{array}$ & \\
\hline BNL & 0.002 & $x$ & $\mathrm{X}$ & $x$ & $x$ & 0.008 \\
\hline $\mathrm{BBH}$ & $x$ & $x$ & $x$ & 0.050 & $x$ & $x$ \\
\hline WFB & 0.006 & $x$ & $x$ & 0.006 & $x$ & $x$ \\
\hline ZYB & 0.001 & $x$ & $x$ & 0.007 & $x$ & $x$ \\
\hline AUB & 0.004 & $x$ & $x$ & 0.038 & $x$ & $x$ \\
\hline $\mathrm{NPH}^{2}$ & 0.001 & $x$ & $x$ & $\mathrm{x}$ & $x$ & $x$ \\
\hline $\mathrm{NLH}$ & 0.008 & $x$ & $x$ & $x$ & $x$ & $x$ \\
\hline JUB & 0.001 & $x$ & $x$ & $x$ & $x$ & $x$ \\
\hline NLB & 0.009 & $x$ & $x$ & 0.001 & $x$ & $x$ \\
\hline MAB & 0.000 & $x$ & $\mathrm{X}$ & 0.001 & $x$ & $x$ \\
\hline $\mathrm{MDH}$ & 0.014 & $x$ & 0.014 & 0.003 & $x$ & $x$ \\
\hline DKB & 0.002 & $x$ & $X$ & 0.011 & $x$ & $x$ \\
\hline WNB & 0.031 & $x$ & $x$ & $x$ & $x$ & $x$ \\
\hline SIS & 0.039 & $x$ & $x$ & $x$ & 0.049 & 0.003 \\
\hline ZMB & 0.028 & $x$ & $x$ & 0.047 & $x$ & $x$ \\
\hline SSS & $x$ & $x$ & $X$ & 0.004 & $x$ & 0.045 \\
\hline FMB & 0.000 & $x$ & 0.02 & 0.001 & $x$ & $x$ \\
\hline NAS & 0.000 & $x$ & $x$ & 0.027 & $x$ & 0.019 \\
\hline EKB & 0.000 & $x$ & 0.038 & 0.000 & $x$ & $x$ \\
\hline FRC & $x$ & $x$ & $X$ & 0.046 & 0.03 & $x$ \\
\hline FOB & 0.001 & $x$ & 0.018 & $x$ & $x$ & $x$ \\
\hline UFBR & 0.000 & $x$ & 0.013 & 0.000 & $x$ & $x$ \\
\hline GNI & 0.004 & $x$ & $X$ & 0.028 & $x$ & 0.032 \\
\hline HML & 0.001 & $x$ & $x$ & 0.006 & $x$ & 0.037 \\
\hline GOG & 0.005 & 0.037 & 0.002 & $x$ & $x$ & $x$ \\
\hline CDL & 0.009 & 0.009 & $x$ & $x$ & $x$ & $x$ \\
\hline XRH & 0.014 & $x$ & & & $x$ & $x$ \\
\hline MAN & $\mathrm{x}$ & $x$ & $X$ & $x$ & 0.009 & $x$ \\
\hline
\end{tabular}

${ }^{1}$ GT-2 post-hoc tests used except were indicated.

${ }^{2}$ Games-Howell post hoc test used when variance between sub-groups is unequal. 
no change throughout time in the Eastern Cape region for males and females respectively, considering that only two variables for females and six variables for males produce statistically significant differences. However, these findings suggest possible increases in male facial projection and mandibular corpus size with accompanying increases in female mandibular breath through time.

\subsubsection{Multivariate Analysis}

\subsubsection{Principal Components Analysis (PCA)}

The principal component results for each of the cranial variable sets (vault, face and mandible) are handled independently. The principal component results indicate that significant changes in cranial size and shape did not take place through time in the Eastern Cape region. Forty-nine crania were complete enough that the entire suite of variables could be used in the PCA.

The vault variable set produced two principal components, the first representing size (PC1) and the second representing shape (PC2) (Table 4.2). The vault size (PC1) and shape (PC2) scores of the four sub-groups overlap considerably. This indicates that there are no

Table 4.2. Craniometric Principal Component Analysis: Variable sets and corresponding Principal Component loadings

\begin{tabular}{lccccccccc}
\hline Vault & $\begin{array}{c}\text { PC1 } \\
(40.2 \%)^{*}\end{array}$ & $\begin{array}{c}\text { PC2 } \\
(17.3 \%)\end{array}$ & Face & $\begin{array}{c}\text { PC1 } \\
(46.3 \%)\end{array}$ & $\begin{array}{c}\text { PC2 } \\
(17.3 \%)\end{array}$ & $\begin{array}{c}\text { PC3 } \\
(11.8 \%)\end{array}$ & Mandible & $\begin{array}{c}\text { PC1 } \\
(41.6 \%)\end{array}$ & $\begin{array}{c}\text { PC2 } \\
(26.5 \%)\end{array}$ \\
\hline GOL & 0.654 & -0.344 & NLH & 0.598 & 0.535 & -0.385 & GNI & 0.744 & 0.581 \\
BNL & 0.411 & -0.623 & JUB & 0.835 & -0.186 & -0.068 & GOG & 0.422 & 0.743 \\
BBH & 0.874 & 0.109 & NLB & 0.555 & -0.016 & 0.486 & WRB & 0.595 & -0.275 \\
XCB & 0.447 & 0.305 & MAB & 0.658 & -0.267 & 0.166 & XRH & 0.818 & -0.186 \\
WFB & 0.378 & 0.415 & OBH & 0.527 & 0.492 & -0.454 & MXML & 0.721 & -0.188 \\
ASB & 0.522 & 0.256 & OBB & 0.181 & 0.748 & 0.564 & MAN & -0.408 & 0.723 \\
FRC & 0.714 & -0.413 & DKB & 0.6 & -0.523 & -0.096 & & & \\
PAC & 0.578 & 0.439 & FMB & 0.816 & -0.038 & -0.101 & & & \\
& & & EKB & 0.9 & -0.033 & 0.205 & & & \\
\hline
\end{tabular}

* Number in bracket is the percentage variance explained by each component 
Figure 4.1. Scatterplot of PC1 and 2 scores for vault variables

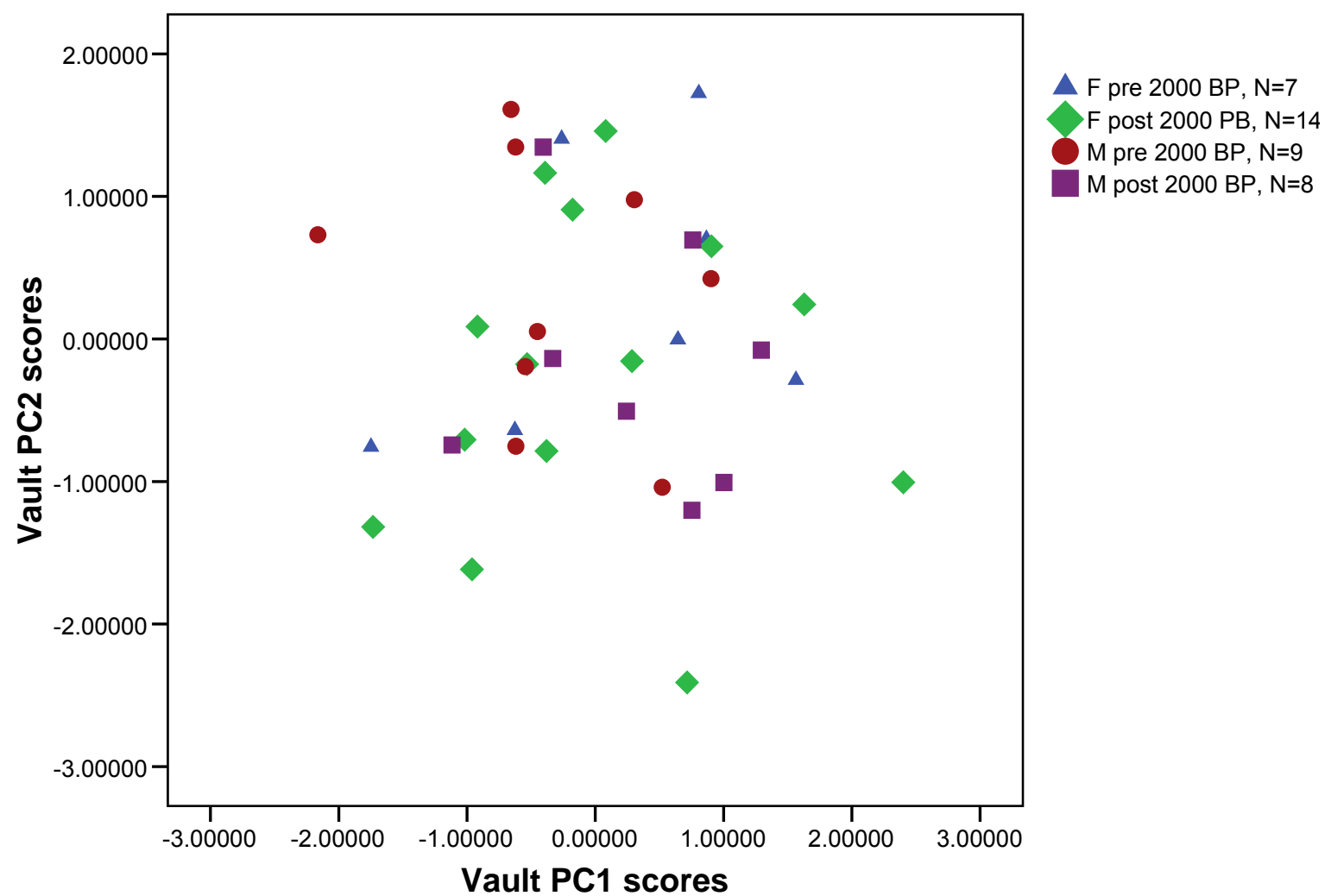

significant cranial differences through time (Fig. 4.1). The vault PC2 scores for the pre-2000 $\mathrm{BP}$ males and females and the post-2000 BP males cluster together and range from -1.0 to + 1.5. The post-2000 BP females also fall within this grouping, but the scores for this group are more variable, with a few scores falling between -1.0 and -2.5 . A small number of females in the post-2000 BP group have large crania that fall within the male range for this period. It is probable that the greater range of the cranial vault values for females dating to the last 500 years is responsible for the variability in post-2000BP female PC2 scores.

More separation of the four subgroups is evident in the face principal component scores, yet the scores for all subgroups still overlap considerably. With the exception of the post-2000 BP females, the remaining subgroups cluster along temporal lines. The pre-2000 BP males and females fall on the negative end of the PC1 axis while the post -2000 BP males 
Figure 4.2. Scatterplot of PC1 and 2 scores for face variables

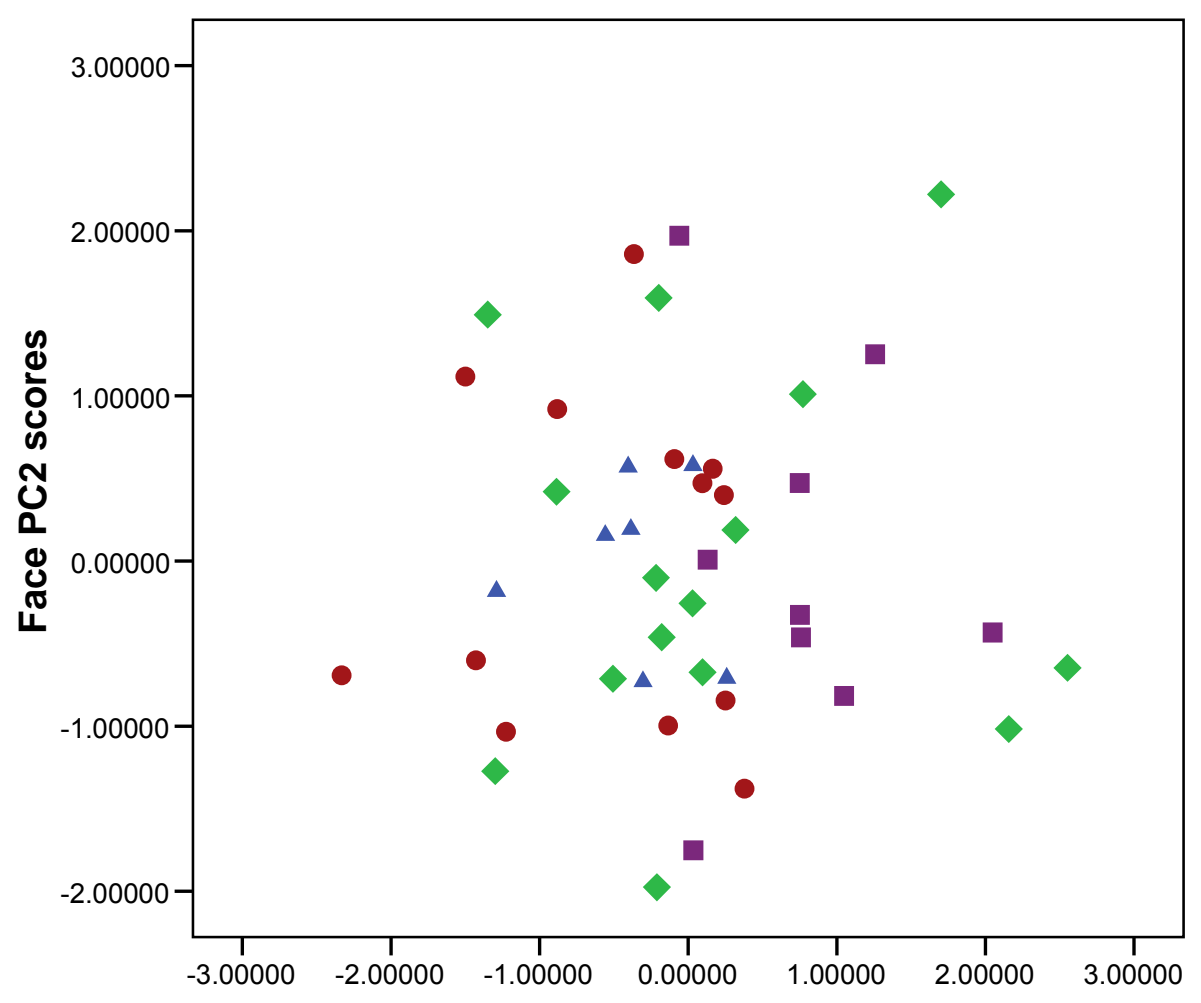

F pre $2000 \mathrm{BP}, \mathrm{N}=7$ $F$ post $2000 \mathrm{BP}, \mathrm{N}=15$ $M$ pre $2000 \mathrm{BP}, \mathrm{N}=13$

$M$ post $2000 \mathrm{BP}, \mathrm{N}=9$

Face PC1 scores

Figure 4.3. Scatterplot of PC1 and 3 scores for face variables

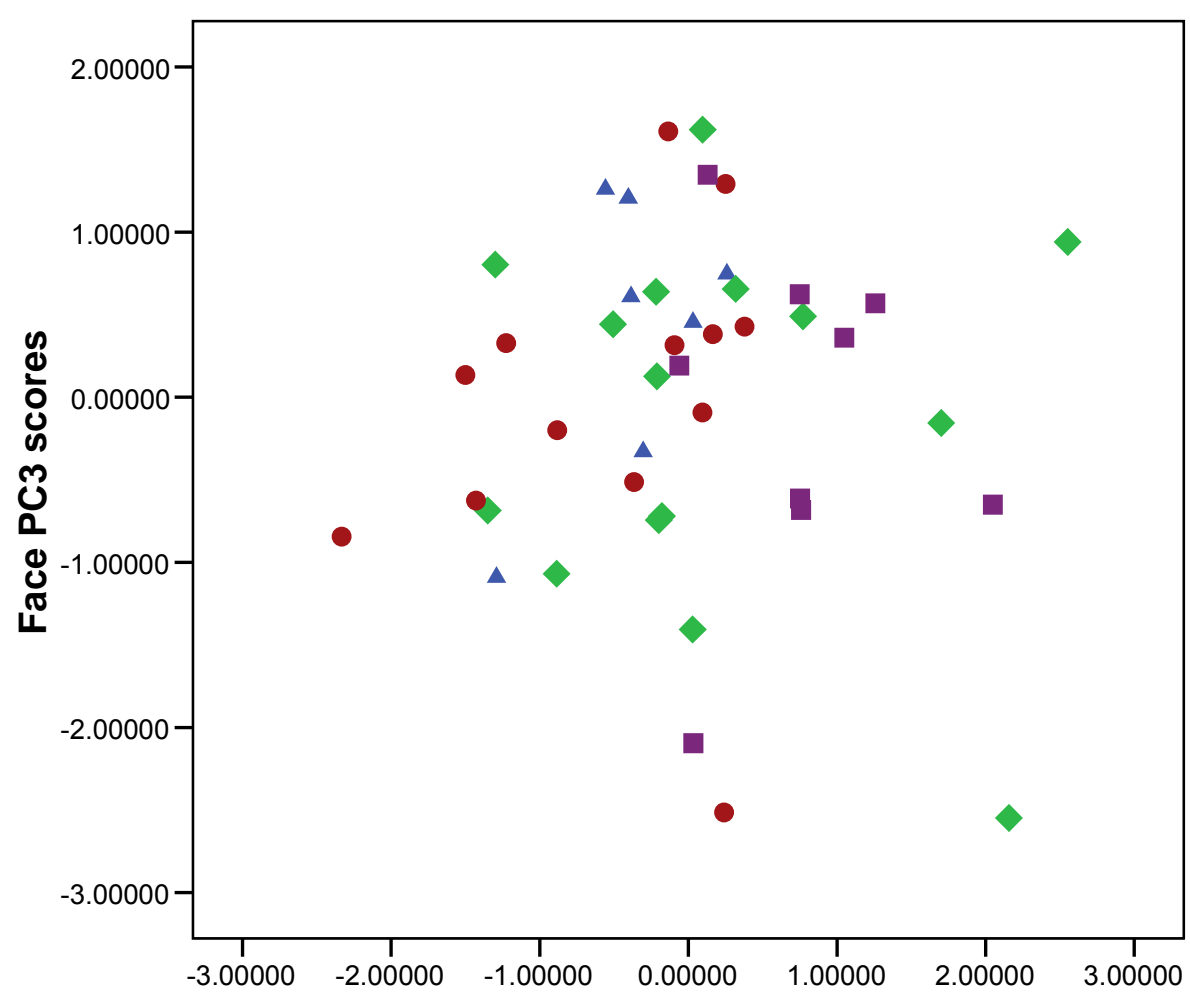

A F pre $2000 \mathrm{BP}, \mathrm{N}=7$ $F$ post $2000 \mathrm{BP}, \mathrm{N}=15$

$\mathrm{M}$ pre $2000 \mathrm{BP}, \mathrm{N}=13$

$M$ post $2000 \mathrm{BP}, \mathrm{N}=9$

Face PC1 scores 
lie at the positive end, suggesting a general increase in facial size through time (Figure 4.2). The post-2000 BP female group is again the most variable, spanning the entire $\mathrm{PC} 1$ axis, thus reinforcing the variability in cranial size of this sub-group. No differences in the PC2 shape scores are evident; the pre-2000 BP and post-2000 BP males overlap and exhibit the same range of scores as the post $2000 \mathrm{BP}$ females. The pre-2000 BP female PC2 scores cluster around zero and range only between -1.0 and +1.0 . Based on these results it is not possible to deduce any changes in facial shape through time. The third principal component does not provide any further insight into possible face shape changes as there is significant overlap of the scores for all four subgroups (Figure 4.3).

The mandibular principal component results follow the same pattern as those characterizing the rest of the cranium (Figure 4.4). The pre-2000 BP female PC1 scores are the most tightly clustered, suggesting that this group may be the most uniform or homogeneous, yet they fall between and overlap with the male PC1 scores. The pre-2000 BP male scores generally fall at the negative end of the PC1 axis and the post-2000 BP scores at the positive end of the axis, with some scores from both sub-groups overlapping around zero. These findings suggest a slight temporal shift toward increasing mandibular size. The range of variability of the female post-2000 BP PC1 and PC2 scores is in keeping with the vault and face principal component results. The PC2 scores for all four sub-groups overlap considerably and span a wide range of values. The pre-2000 BP females are an exception to this. They form the tightest cluster of scores that all fall in the negative end of the $\mathrm{PC} 2$ range. 
In summary, the findings of the principal component analysis indicate that the pre$2000 \mathrm{BP}$ females are the most uniform and the post-2000 BP females are the least uniform of the four sub-groups. Increases in cranial size are evident in both sexes.

Figure 4.4. Scatterplot of PC1 and 2 scores for mandibular variables

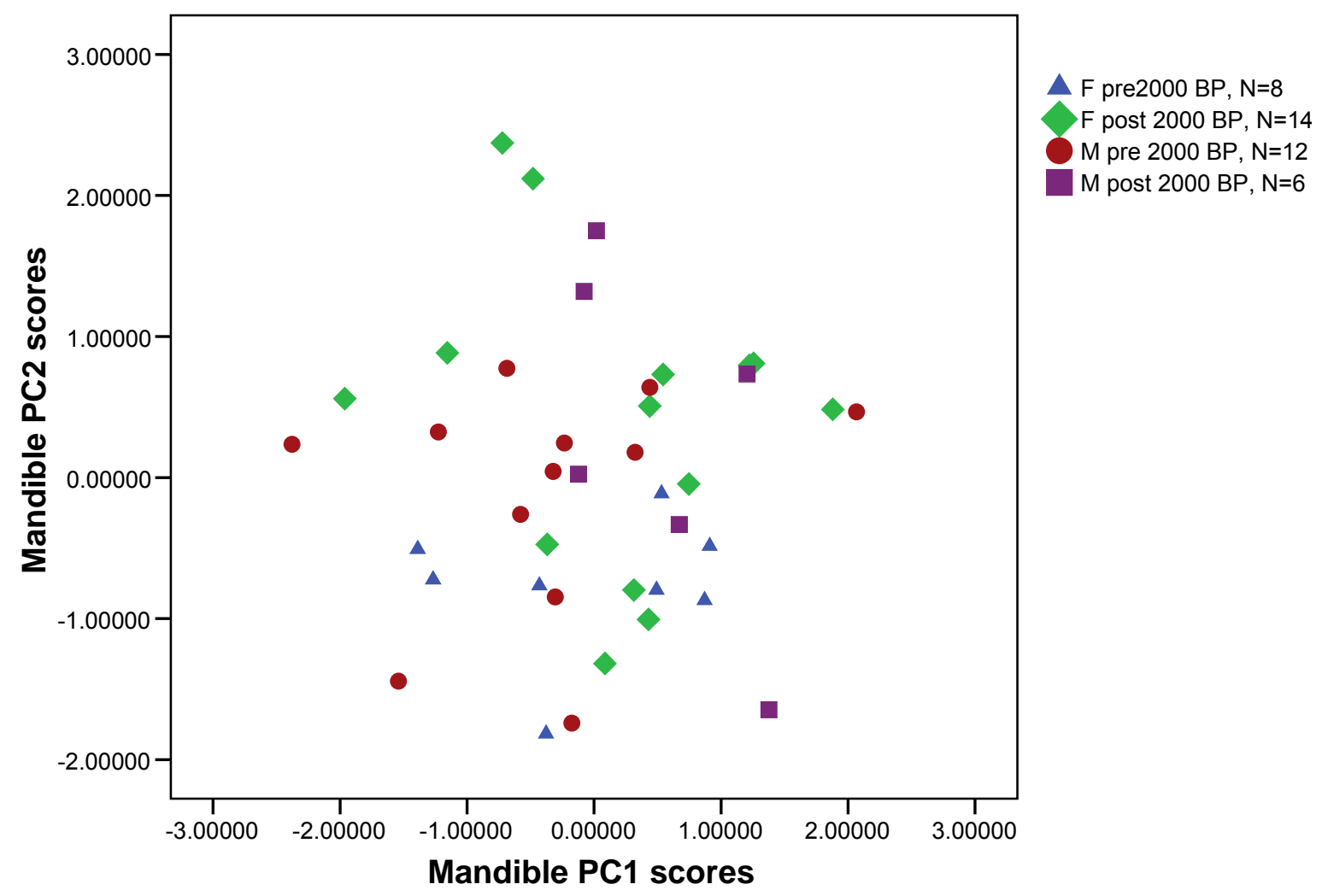

\subsubsection{Curve Estimation Regression}

The PC scores were regressed on radiocarbon date in an attempt to identify trends relating to cranial size and shape through time. The regression results corroborate those of the principal components analysis, but provide a clearer picture of cranial change through time. Although the slopes of the regression of the vault PC1 scores against radiocarbon date do not denote a significant change in vault size through time for males $\left(r^{2}=0.093, p=0.504\right)$ or females $\left(r^{2}=0.086, p=0.444\right)$, cranial vault size appears to become more variable 
between 3500 to 2000 BP (Figure 4.5). Post-2000 BP, most data points fall within the pre3500 BP range. While a considerable amount of variability continued, individuals with small vaults of the magnitude observed between 3500 and $2000 \mathrm{BP}$ are not present after approximately $1800 \mathrm{BP}$. This observed increase in variability must be viewed critically as it may simply be a product of a larger post-2000 BP sample size, since few individuals date to pre-4000 BP. Changes in shape are observed in the male vault, with a decrease in breadth and an increase in length $\left(\mathrm{r}^{2}=0.395, \mathrm{p}=0.007\right)$. A similar pattern is observed for females, but this relationship is not statistically supported $\left(r^{2}=0.156, p=0.218\right)$. Although the relationship between time and change in vault shape is statistically supported, this relationship is weak and begins to take place prior to the 2000 BP benchmark (Figure 4.6).

Figure 4.5. Scatterplot of regression scores for vault variables: $\mathrm{PC} 1 \mathrm{vs} .{ }^{14} \mathrm{C}$.

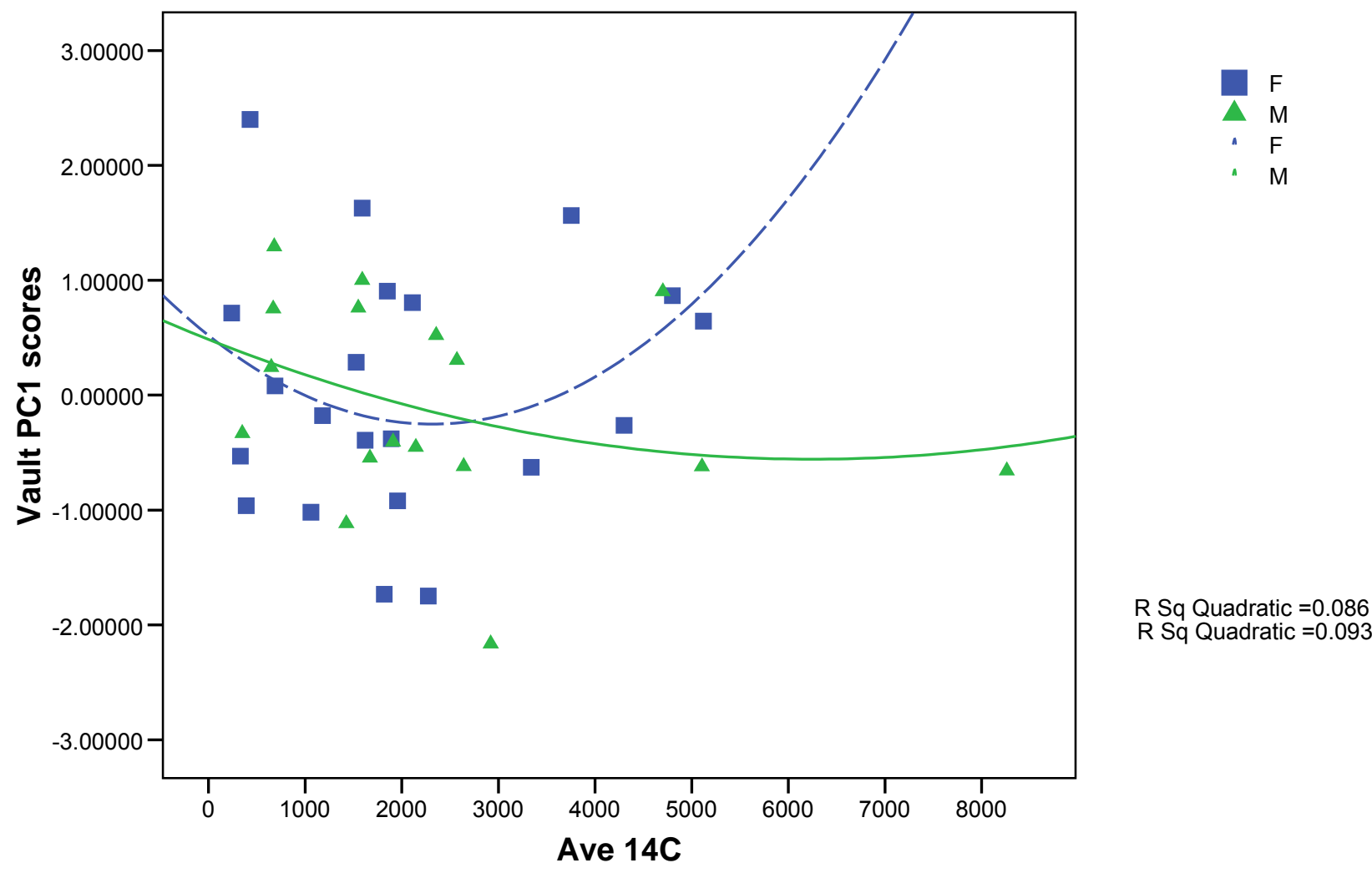


Figure 4.6. Scatterplot of regression scores for vault variables: PC2 vs. ${ }^{14} \mathrm{C}$.

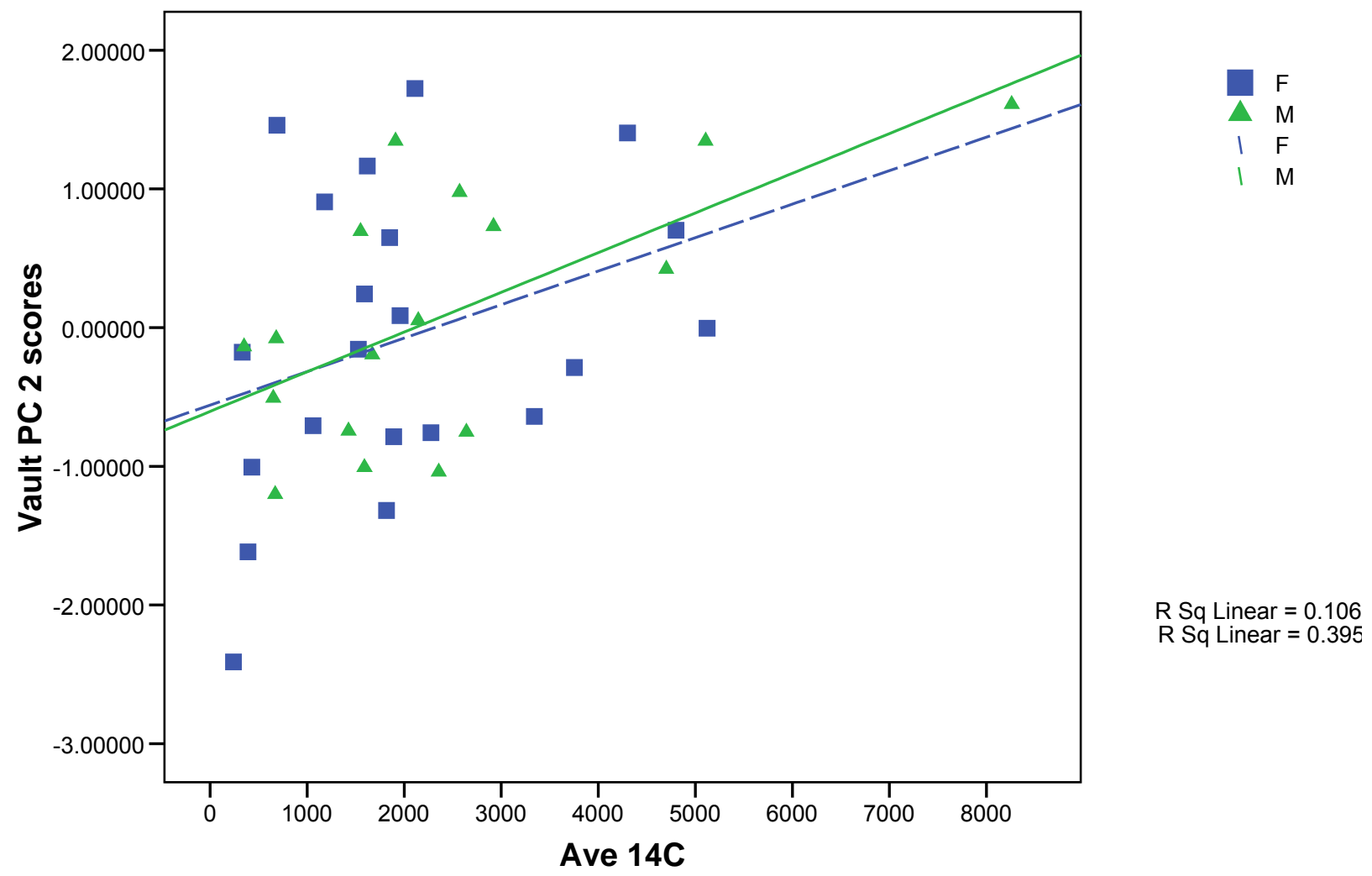

Male face size exhibits interesting changes through time. The regression value suggests a significant reduction in the size of male faces through time $\left(r^{2}=0.507, p=0.001\right)$. Pre-2000 BP male facial size remains stable until around 3000 BP when the males with the smallest faces are observed. Just prior to 2000 BP male face size increases significantly, returning to the maximum of the pre-3500 size range, and continues to increase into the recent past (Fig. 4.7). It is possible that a few males dating to around $3000 \mathrm{BP}$ with very negative (small) face PC1 scores may be enhancing the regression curve. Nevertheless, a localized decrease in facial size, even if only among a few individuals, is notable. A similar situation is not observed for females $\left(r^{2}=0.096, p=0.160\right)$. Female facial size appears to remain stable through time, becoming more variable just after $2000 \mathrm{BP}$, with the addition of individuals with larger faces. The small size of the female sample postdating $2000 \mathrm{BP}$ 
(female $\mathrm{n}=7$ while male $\mathrm{n}=12$ ) may be artificially enhancing the apparent stability in facial size during pre-2000 BP period.

Figure 4.7. Scatterplot of regression scores for face variables: PC1 vs. ${ }^{14} \mathrm{C}$.

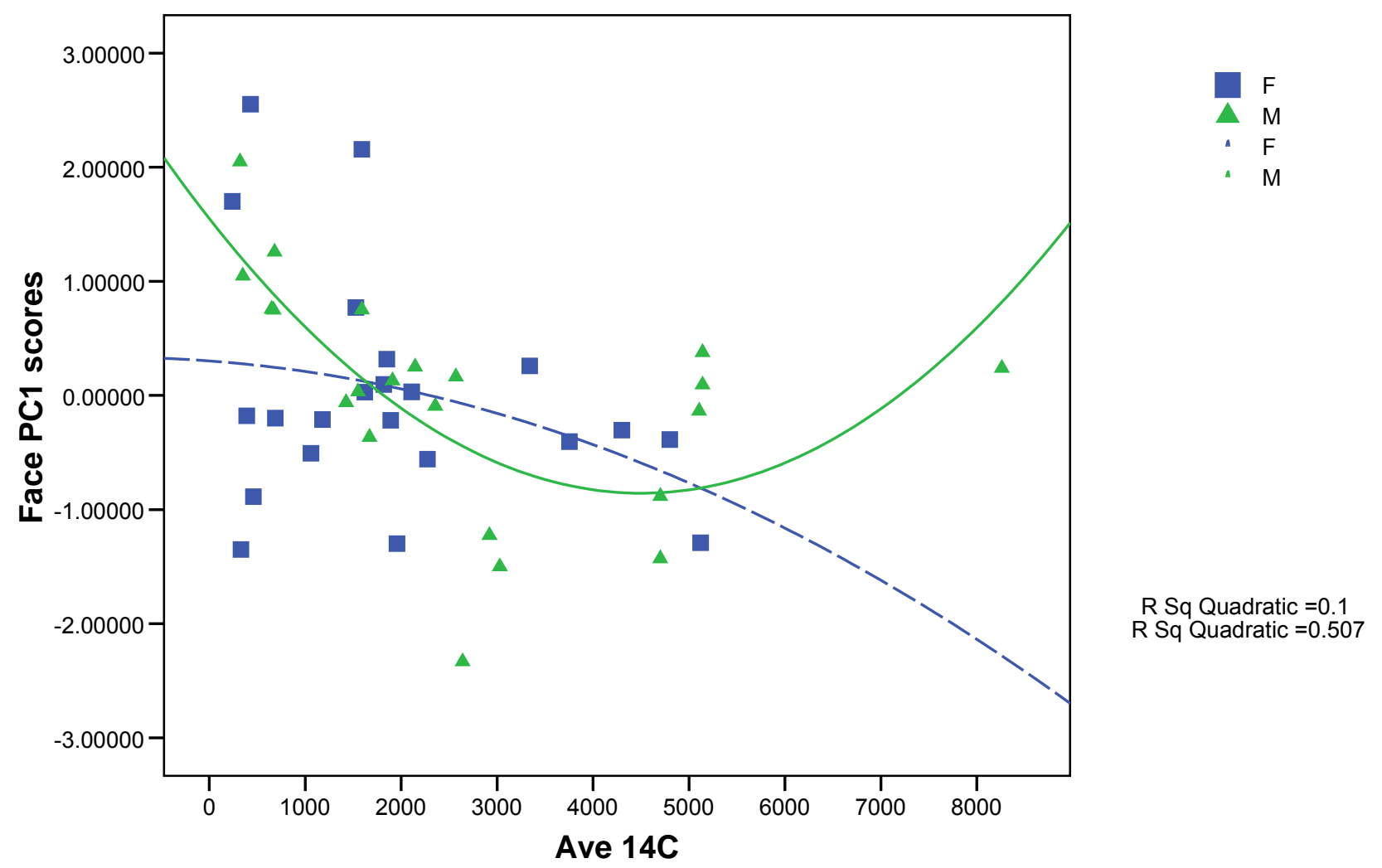

Statistically significant relationships are not supported between face shape and time for females $\left(\mathrm{r}^{2}=0.149, \mathrm{p}=0.216\right)$ or males $\left(\mathrm{r}^{2}=0.010, \mathrm{p}=0.909\right)$. Females display a slight change in facial shape between 3500 to $2000 \mathrm{BP}$, but this is not significant. Male face shape appears to be somewhat uniform prior to $2000 \mathrm{BP}$, becoming more variable around $2000 \mathrm{BP}$ (Figure 4.8). In keeping with the PCA results, regression of the face PC3 scores against the ${ }^{14} \mathrm{C}$ data does not produce significant results for females $\left(\mathrm{r}^{2}=0.161, \mathrm{p}=0.188\right)$ or males $\left(\mathrm{r}^{2}=\right.$ $0.288, \mathrm{p}=0.059)($ Figure 4.9). 
Figure 4.8. Scatterplot of regression scores for face variables: PC2 vs. ${ }^{14} \mathrm{C}$.

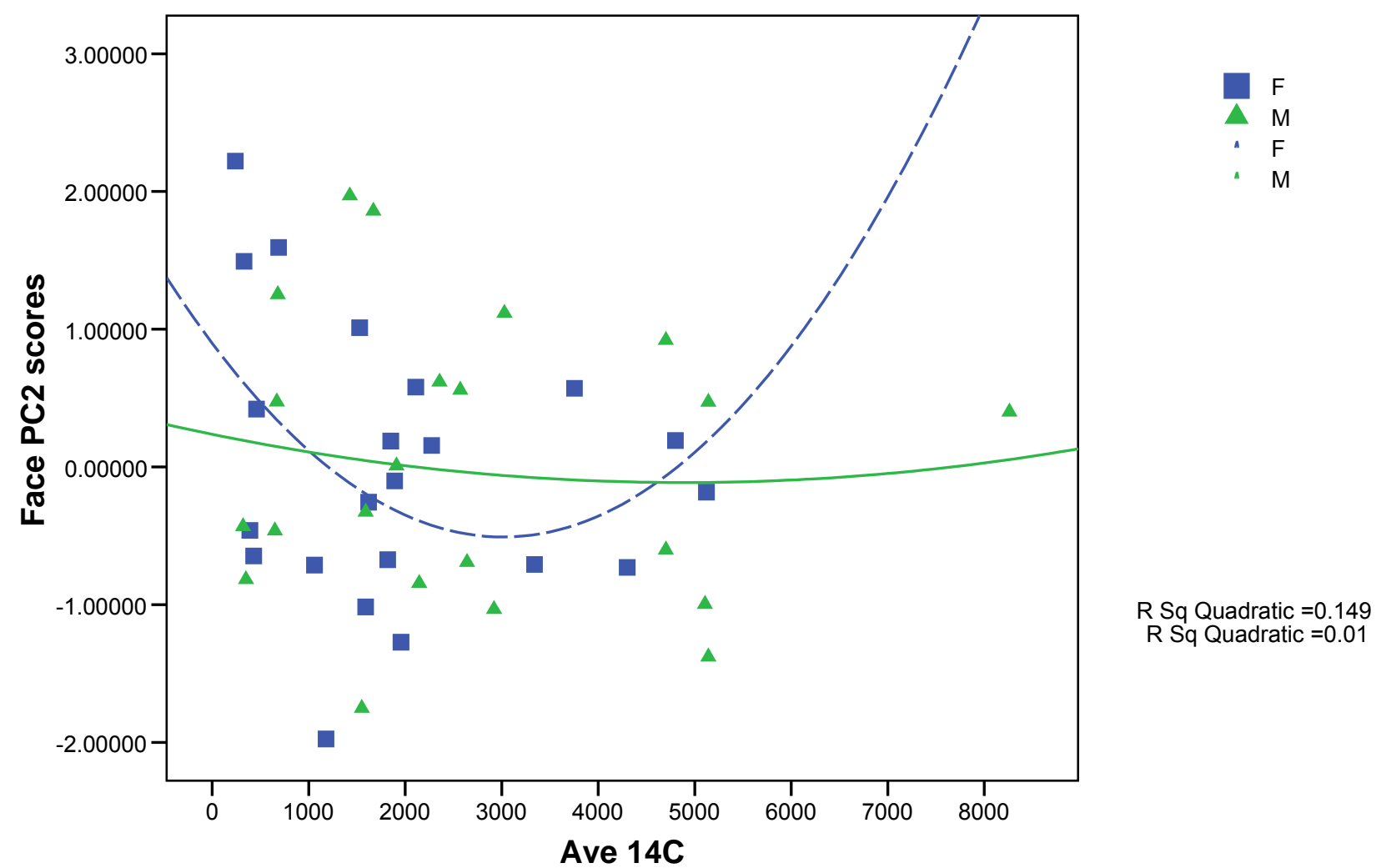

Figure 4.9. Scatterplot of regression scores for face variables: PC3 vs. ${ }^{14} \mathrm{C}$.

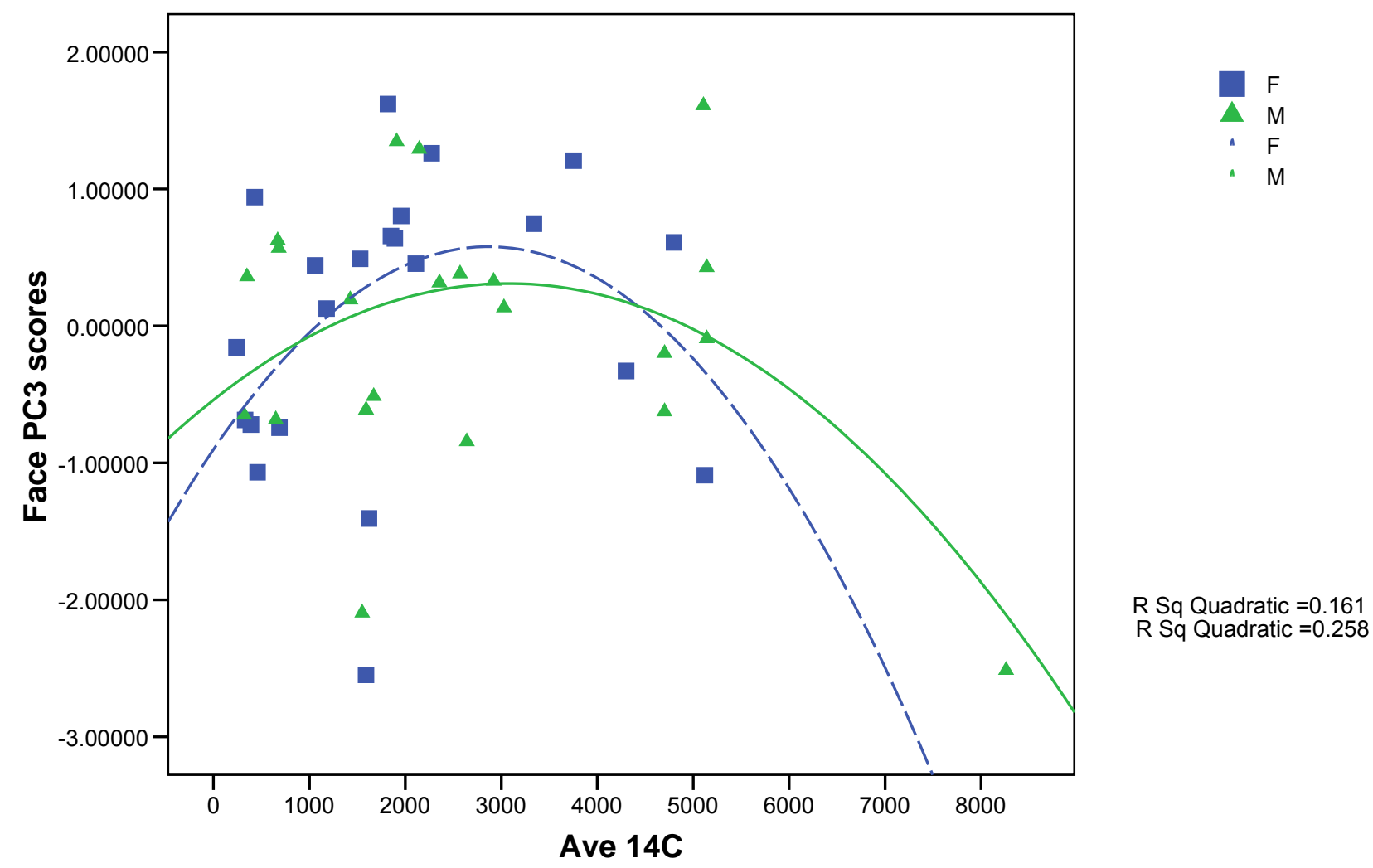


Mandibular size remains relatively uniform through time. No statistically supported relationships occur for females $\left(r^{2}=0.018, p=0.548\right)$ or males $\left(r^{2}=0.137, p=0.330\right)$ (Figure 4.10). Mandibular size appears to become less variable between 4000 and 2000 BP. Prior to 4000 BP mandibular size ranges from very large and very small (high positive and high negative PC1 scores, respectively). Just prior to 2000 BP it seems that male and female mandibular size increases, with the absence of mandibles falling at the small end of the range observed previously. Within the last 2000 years, male mandibular size appears to remain stable, while female size becomes more variable with the advent of some individuals with very large and very small mandibles in the last 500 years.

Figure 4.10. Scatterplot of regression scores for mandibular variables: PC1 vs. ${ }^{14} \mathrm{C}$.

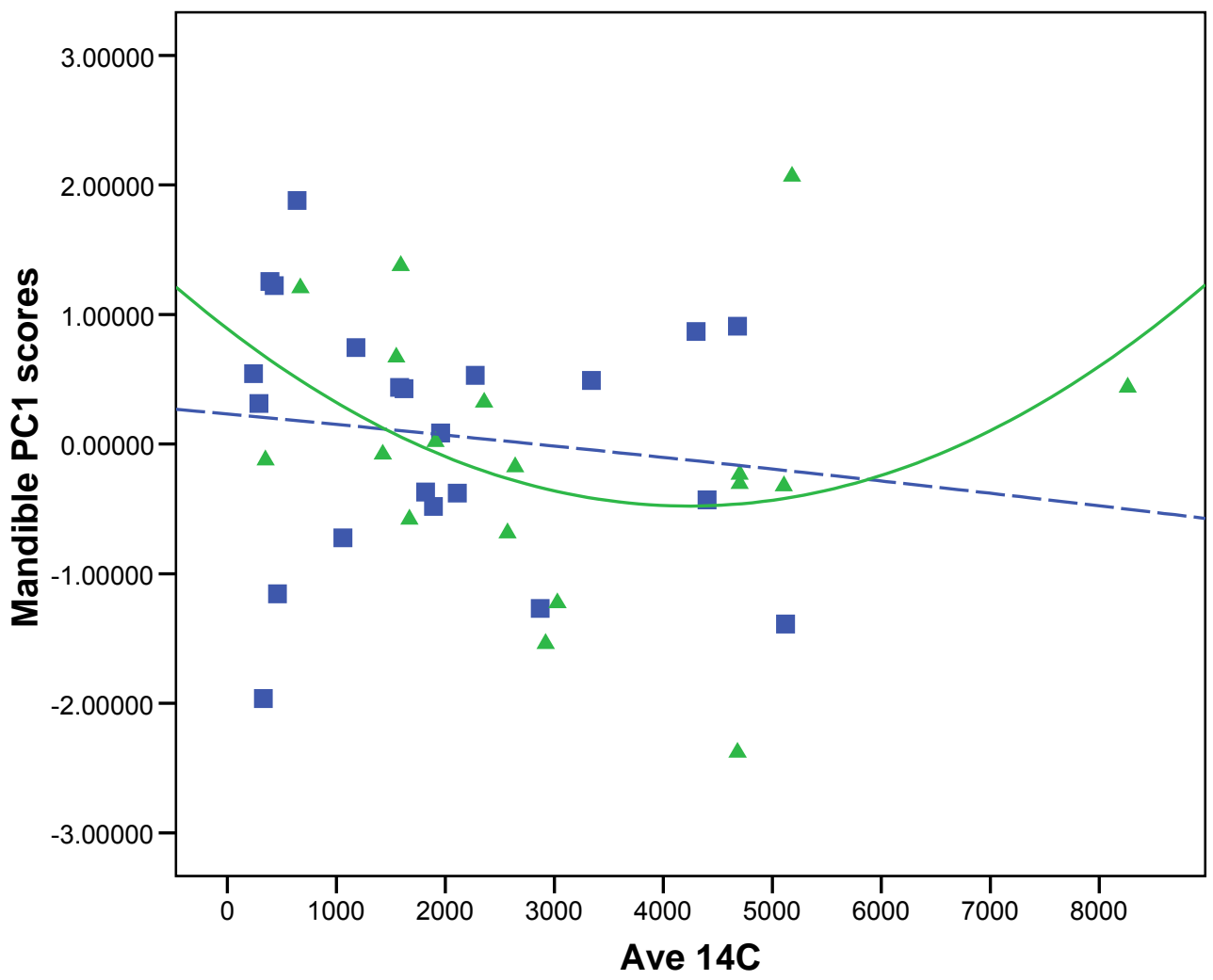


Statistically supported changes in mandibular shape are observed only for females $\left(\mathrm{r}^{2}\right.$ $=0.222, \mathrm{p}=0.027)$ where width increases while length and height decrease through time (Figure 4.11). Female mandibular shape appears to remain relatively stable, but changes markedly just prior to $2000 \mathrm{BP}$. The magnitude of the female mandibular shape changes may be influenced partly by the small size of the pre-2000 BP sample. Male mandibular shape increases in variability around 3500BP, becoming even more variable prior to and following $2000 \mathrm{BP}$, but this trend is not statistically supported. In general, the results indicate that cranial size and shape became more variable just prior to $2000 \mathrm{BP}$, with variability increasing in magnitude in the last 500 years.

Figure 4.11. Scatterplot of regression scores for mandibular variables: PC2 vs. ${ }^{14} \mathrm{C}$.

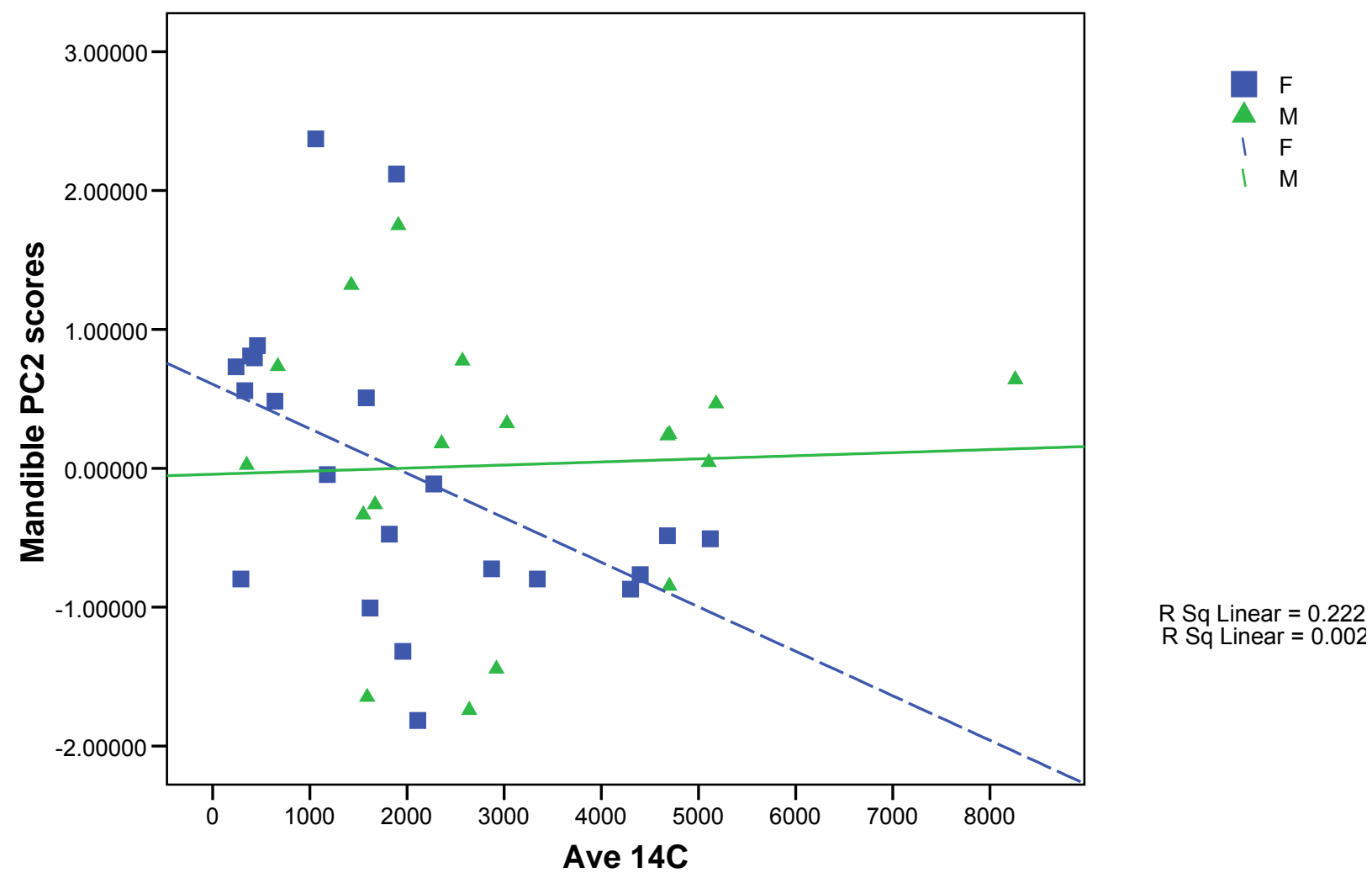




\subsubsection{Comparison with Other Sub-Saharan African Samples}

Comparisons are made between the Eastern Cape and sub-Saharan archaeological male and female samples for a selection of craniometric variables (Tables 4.3 and 4.4). The Eastern Cape sample is divided into the four sub-groupings that were used in the multivariate analysis. T-tests are used to make comparisons with published summary data (mean and standard deviation). In general, Eastern Cape males appear to be more similar to the male comparative samples than females are to the comparative females. Although a greater number of craniometric variables are significantly different between the pre-2000 BP males and the comparative samples than the post-2000 BP males, the proportion of significant differences is lower than for females. Comparisons between the post-2000 males and the Riet River, Kakamas, Abrahamsdam and Griqua males do not produce any significant differences, suggesting that the post-2000 BP males are most similar to the Orange River samples. Conversely, post-2000 BP males are least similar to Central African Bantu, WestCentral African, East African and South African Bantu males (Table 4.3). Differences between the post-2000 BP males and the comparative groups are mainly restricted to the size of the orbits and cranial height.

Examining pre-2000 BP males in comparison to the other African male samples, the greatest number of significant differences occur between the West-Central African and Central African Bantu males ( $\mathrm{n}=8)$, followed closely by the South African Bantu $(\mathrm{n}=7)$, and then the Kakamas and East African males $(n=6)$ (Table 4.3). Most of the differences occur in the nasal and orbital region, with few differences among the cranial vault variables. The pre2000 BP males are most similar to the South African Khoesan, Riet River, and Abrahamsdam 


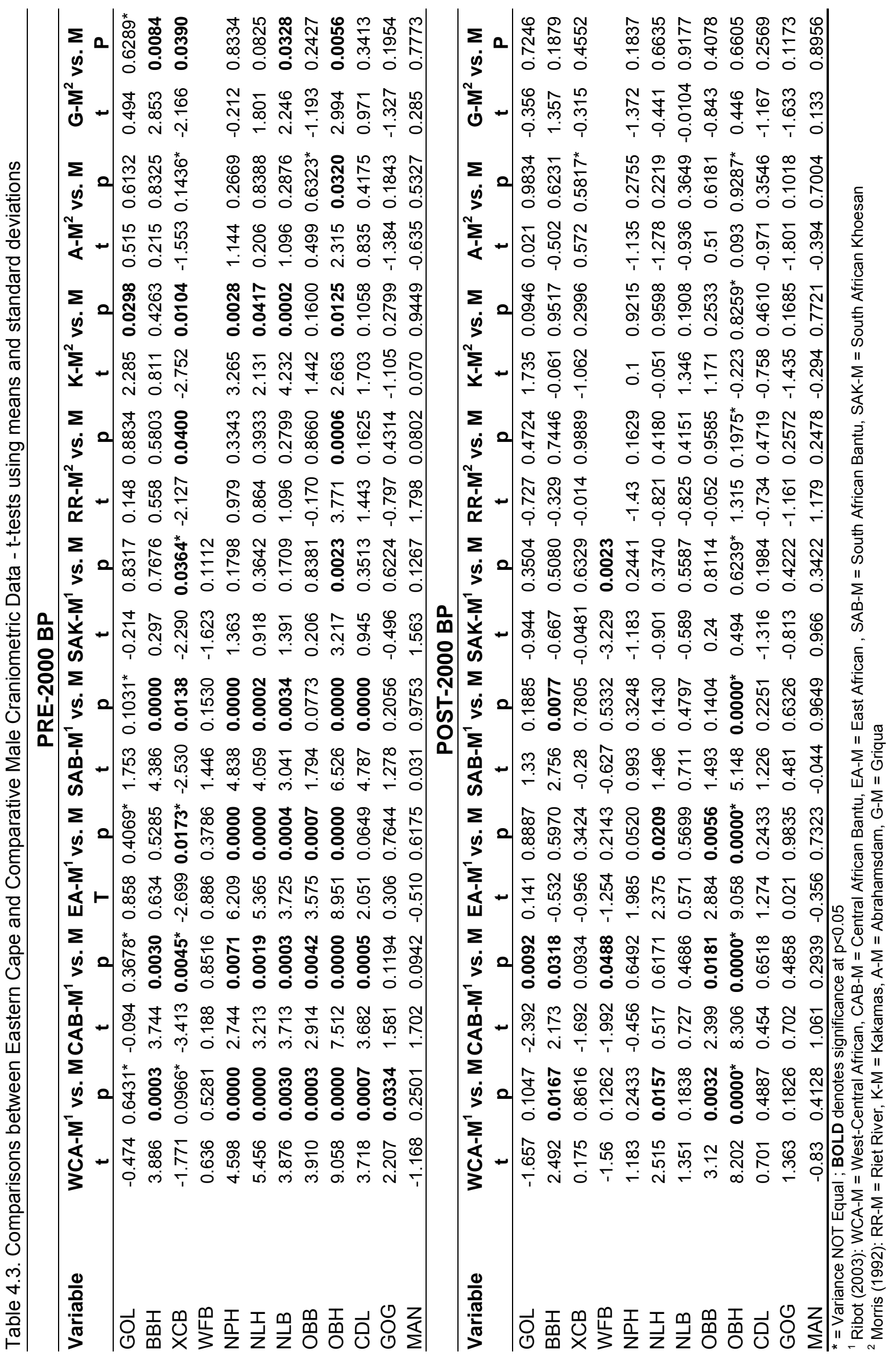




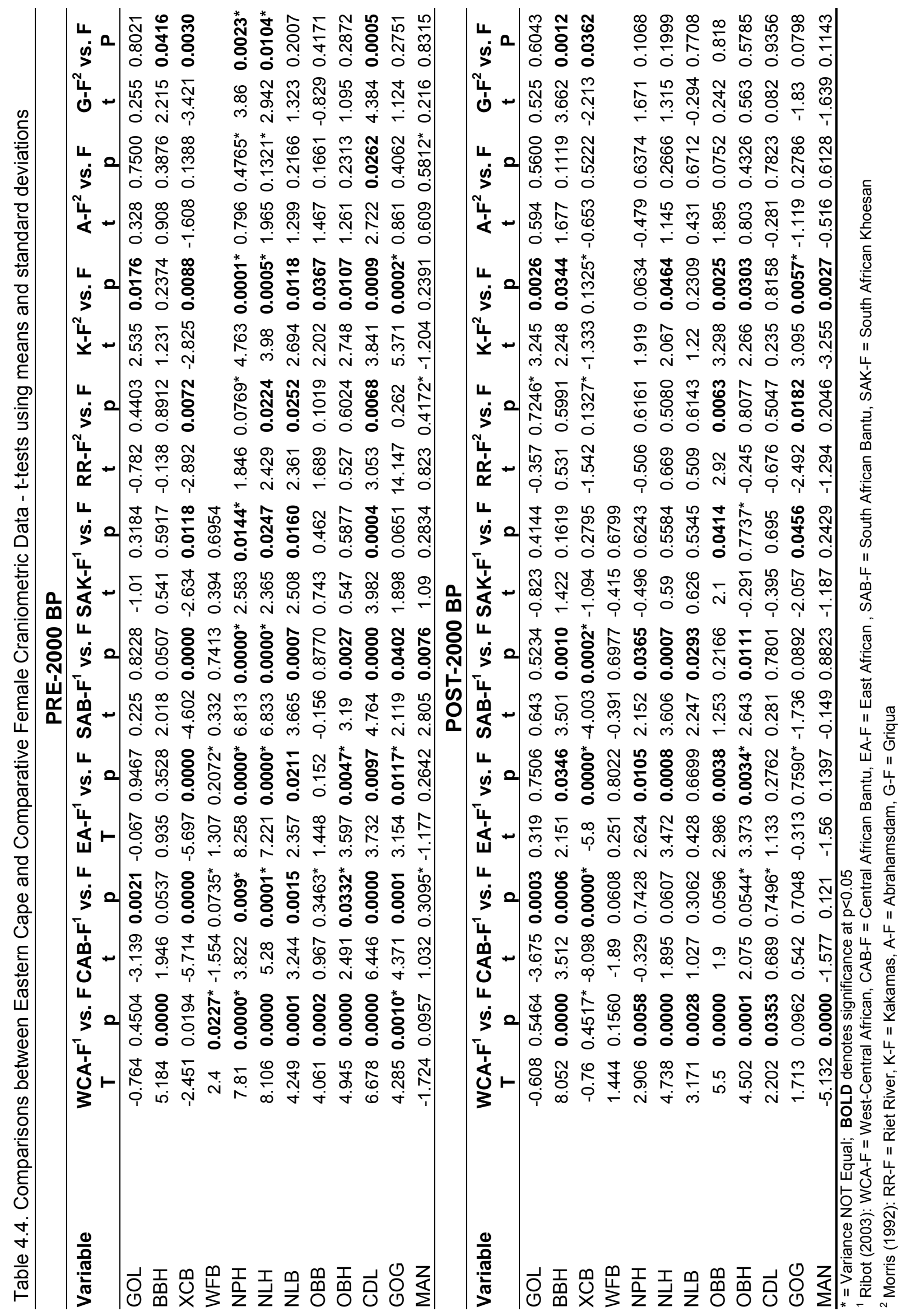


males. Differences between the Eastern Cape males and these groups are limited to the orbital height and cranial breadth variables.

Pre-2000 BP females are the most distinctive relative to the comparative samples. Pre-2000 BP females are most similar to the Abrahamsdam females with only one craniometric variable, bicondylar width of the mandible, producing a statistically significant difference. On the other hand, the large number of significant t-test values suggests that the pre-2000 BP females are least similar to the West-Central African and Kakamas (n=9), followed by the South African Bantu (n=8), and then the Central and East African ( $n=7)$ females (Table 4.4).

Post -2000 BP female comparisons follow the same pattern as the pre-2000 BP females, yet the number of significant differences is considerably lower. Like the males and pre-2000 BP females, the post-2000 BP females exhibit the most affinity in cranial size with the Abrahamsdam sample (Table 4.4). Pre-2000 BP females are least similar to the WestCentral African females, followed by the Kakamas, East African and South African Bantu. Unlike the other three Eastern Cape sub-groups, cranial size differences between the post$2000 \mathrm{BP}$ and comparative females do not follow a consistent pattern.

In summary, significant differences in cranial size are observed between the Eastern Cape and the comparative Bantu-speaking samples. The greatest degree of cranial similarity is observed between the Eastern Cape sample and the South African Khoesan and forager and herder groups from the Orange River area. Although the magnitude of cranial size differences is greater for Eastern Cape females, the comparative pattern is similar across the entire Eastern Cape sample. 


\subsection{Odontometrics}

\subsubsection{Univariate Analysis}

\subsubsection{Maximum Dimensions Method}

Because of the extent of wear in this sample and the requirements for recording maximum crown dimensions, sample sizes for maximum buccolingual (BL) and mesiodistal (MD) dimensions are relatively small (see Appendix 8). Maximum dimensions odontometric variables are analyzed using only univariate statistics because meaningful results could not be obtained when the sample was further divided into the anatomical, sex and temporal subgroupings for the multivariate analysis. The extensive occlusal and interstitial wear exhibited by the Eastern Cape sample resulted in very small sample sizes for the maximum mesiodistal dimensions for all dental variables. Of the fifty-seven individuals in the Eastern Cape sample with dental remains, maximum odontometric dimensions could only be collected on 388 teeth from forty-four individuals. If the maximum odontometric sample was further divided into the subgroupings that are required for the multivariate analyses, some subgroups would have included only one or two individuals. Multivariate results based on such small sample sizes would not have produced meaningful results.

Prior to the univariate analysis each maximum dimension odontometric variable (i.e. UI1MBL, UI1MMD, etc.) was plotted against time in order to look for any general differences. Because of the small number of measurements available for each mesiodistal odontometric variable, only the buccolingual dimension is examined. A general decrease in tooth size over time is detected for most of the maximum buccolingual odontometric variables. Slight to moderate decreases in size were observed for both male and female maxillary anterior teeth and molars, and mandibular canines, premolars and molars. On the 
other hand, maxillary second molars exhibit moderate increases in size for both sexes.

Increases in size of the mandibular anterior teeth are observed for females, while decreases are noted for males.

One-way ANOVAs were performed on the maximum dimension odontometric data. Third molars were excluded in the analysis due to their variable nature. Maximum mesiodistal measurements were excluded from the univariate analysis because post-hoc tests used to determine which sub-groups differ require that groups have more than two cases, and when divided, most of the maximum mesiodistal variables contained fewer than two cases per sub-grouping. The ANOVA results do not indicate any significant changes in the sized of the dentition over time. Post-hoc tests reveal that means differ between the four subgroups for only five out of fourteen maximum buccolingual odontometric variables: maxillary second premolars, maxillary second molars, mandibular second incisors, and mandibular first and second molars (Table 4.5). The pattern of size differences is reversed from that observed in the craniometric univariate analysis. Post-2000 BP females have the Table 4.5. Probability values for Maximum Buccolingual Odontometric Dimensions Posthoc1 ANOVA tests - variables that differ significantly between sub-groups $(p<0.05)$

\begin{tabular}{|c|c|c|c|c|c|c|}
\hline \multirow{2}{*}{$\begin{array}{c}\text { Maximum } \\
\text { Odontometric } \\
\text { Variables }\end{array}$} & \multicolumn{3}{|c|}{$\begin{array}{c}\text { Pre } 2000 \text { BP } \\
\text { Females }\end{array}$} & \multicolumn{2}{|c|}{$\begin{array}{l}\text { Post } 2000 \text { BP } \\
\text { Females }\end{array}$} & \multirow{2}{*}{$\begin{array}{c}\text { Pre } 2000 \mathrm{BP} \\
\text { Males } \\
\text { vs. } \\
\text { Post } 2000 \mathrm{BP} \\
\text { Males }\end{array}$} \\
\hline & $\begin{array}{c}\text { vs. } \\
\text { Post } 2000 \text { BP } \\
\text { Males }\end{array}$ & $\begin{array}{c}\text { vs. } \\
\text { Post } 2000 \text { BP } \\
\text { Females }\end{array}$ & $\begin{array}{l}\text { vs. } \\
\text { Pre } 2000 \text { BP } \\
\text { Males }\end{array}$ & $\begin{array}{c}\text { vs. } \\
\text { Post } 2000 \text { BP } \\
\text { Males }\end{array}$ & $\begin{array}{l}\text { vs. } \\
\text { Pre } 2000 \\
\text { BP Males }\end{array}$ & \\
\hline UP2BL & 0.026 & $\mathrm{x}$ & $\mathrm{x}$ & $\mathrm{x}$ & $x$ & $\mathrm{x}$ \\
\hline UM2BL & $x$ & $x$ & $x$ & 0.039 & $x$ & $x$ \\
\hline LI2BL $^{2}$ & $x$ & $x$ & 0.005 & $x$ & $x$ & $x$ \\
\hline LMIBL & $x$ & $x$ & $x$ & 0.018 & 0.032 & $x$ \\
\hline LM2BL & $x$ & $x$ & 0.001 & 0.007 & $\mathrm{x}$ & $x$ \\
\hline
\end{tabular}

${ }^{1}$ GT-2 post-hoc tests used except were indicated.

${ }^{2}$ Games-Howell post hoc test used when variance between sub-groups is unequal. 
smallest maximum buccolingual dimensions, and pre-2000 BP males have the largest buccolingual odontometric dimensions. Most of the significant differences occur between the post-2000 BP females and males, with males having significantly larger mean maxillary second molar and mandibular first and second molar buccolingual dimensions than females. Unlike the craniometric univariate results, the magnitude of difference in tooth size is not as great for pre-2000 BP females and post-2000 BP males, considering that difference in tooth size is restricted to a single maxillary variable (second premolar). Coincidentally, none of the variables are significantly different between pre- and post-2000 BP males and females, respectively, indicating that maximum tooth size did not change significantly over time. These findings contradict the patterns that were identified when the raw maximum buccolingual dimensions data were plotted against ${ }^{14} \mathrm{C}$, thus calling into question the strength of the temporal patterns when subjected to statistical scrutiny.

\subsubsection{Cervical Dimensions Method}

Differences in the measurement location and requirements relating to tooth wear allow for the inclusion of a significantly larger portion of the Eastern Cape sample in the cervical dimensions method analysis: 494 teeth from fifty seven individuals (see Appendix 9). The ANOVA of the cervical dimensions odontometric data produce few statistically significant differences between the four sub-groups (Table 4.6). All of the significant maximum odontometric variables are restricted to the mandibular teeth. Pre- and post-2000 BP females differ mainly with the post-2000 BP males. Mean pre-2000 BP female differences are generally limited to the anterior while post-2000 BP female mean differences are restricted to the posterior teeth. These differences are influenced by sex-related size differences and are not likely to reflect a temporal trend among the data. Fewer significant 
differences are observed between pre-2000 BP mean values and pre-2000 (first incisor and second molar buccolingual dimensions) and post-2000 (second molar buccolingual dimension and crown area) females. Males and females do not differ significantly in their respective pre- and post-2000 BP groupings. Like the maximum crown dimensions, tooth size at the level of the cemento-enamel junction does not change significantly over time. However, the pattern of cervical odontometric sex-related size differences is the reverse of the maximum crown dimensions. It seems that male maximum buccolingual odontometric dimensions are generally larger prior to $2000 \mathrm{BP}$, but male cervical odontometric dimensions in both planes are larger after $2000 \mathrm{BP}$.

Table 4.6. Probability values for Cervical Odontometric Post-hoc1 ANOVA tests - variables that differ significantly between sub-groups $(p<0.05)$

\begin{tabular}{|c|c|c|c|c|c|c|}
\hline \multirow[b]{2}{*}{$\begin{array}{c}\text { Cervical } \\
\text { Odontometric } \\
\text { Variables }\end{array}$} & \multicolumn{3}{|c|}{ Pre 2000 BP Females } & \multicolumn{2}{|c|}{ Post 2000 BP Females } & \multirow{2}{*}{$\begin{array}{c}\text { Pre } 2000 \text { BP Males } \\
\text { vs. } \\
\text { Post } \\
2000 \text { BP } \\
\text { Males }\end{array}$} \\
\hline & $\begin{array}{c}\text { Vs. } \\
\text { Post } \\
2000 \text { BP } \\
\text { Males }\end{array}$ & $\begin{array}{c}\text { vs. } \\
\text { Post } \\
2000 \text { BP } \\
\text { Females }\end{array}$ & $\begin{array}{c}\text { Vs. } \\
\text { Pre } \\
2000 \text { BP } \\
\text { Males }\end{array}$ & $\begin{array}{l}\text { vs. } \\
\text { Post } \\
2000 \text { BP } \\
\text { Males }\end{array}$ & $\begin{array}{l}\text { vs. } \\
\text { Pre } \\
2000 \text { BP } \\
\text { Males }\end{array}$ & \\
\hline $\mathrm{LI}_{1} \mathrm{CBL}^{2}$ & 0.016 & $\mathrm{x}$ & 0.007 & $x$ & $x$ & $\mathrm{x}$ \\
\hline $\mathrm{LI}^{2} \mathrm{CBL}^{2}$ & 0.024 & $x$ & $x$ & $x$ & $x$ & $x$ \\
\hline LCCBL & 0.023 & $x$ & $x$ & $x$ & $x$ & $x$ \\
\hline LP2CBL & 0.031 & $x$ & $x$ & $x$ & $X$ & $x$ \\
\hline LMICCRA & 0.034 & $x$ & $x$ & 0.012 & $x$ & $x$ \\
\hline LM2CBL & $x$ & $x$ & 0.009 & 0.001 & 0.001 & $x$ \\
\hline LM2CMD & $x$ & $x$ & $\mathrm{x}$ & 0.002 & $\mathrm{X}$ & $x$ \\
\hline LM2CCRA & $x$ & $x$ & $x$ & 0.002 & 0.009 & $\mathrm{x}$ \\
\hline
\end{tabular}

${ }^{1}$ GT-2 post-hoc tests used except were indicated.

${ }^{2}$ Games-Howell post hoc test used when variance between sub-groups is unequal.

\subsubsection{Multivariate Analysis}

\subsubsection{Principal Components Analysis of Cervical Dimensions}

The principal component results are discussed separately for each of the cervical dimension odontometric variable sets. The division of cervical odontometric data into 
maxillary anterior and posterior and mandibular anterior and posterior datasets enabled in the inclusion of 34 individuals in the principal component analysis.

Unlike the craniometric principal components results, the analysis of the cervical odontometric dimensions does not yield any noteworthy results. The maxillary anterior and posterior variable sets both produce two principal components representing size (PC1) and shape (PC2) (Table 4.7). No significant patterns are evident for the maxillary anterior and posterior teeth PC scores; there is considerable overlap among the sub-groups for both the size (PC1) and shape (PC2) components (Figures 4.12 and 4.13). The principal component scores for the maxillary anterior teeth are scattered across the PC1 and PC2 axes and do not conform to any meaningful patterns. While the maxillary posterior principal component scores are more clustered, separation of the four subgroups is not evident. Small sample Table 4.7. Cervical Odontometric variable sets and corresponding Principal Component loadings

\begin{tabular}{|c|c|c|c|c|c|c|}
\hline \multicolumn{7}{|c|}{ Maxillary } \\
\hline Anterior & $\begin{array}{c}\text { PC1 } \\
(69.8 \%)^{*}\end{array}$ & $\begin{array}{c}\text { PC2 } \\
(19.4 \%)\end{array}$ & Posterior & $\begin{array}{c}\text { PC1 } \\
(51.9 \%)\end{array}$ & $\begin{array}{c}\text { PC2 } \\
(15.9 \%)\end{array}$ & \\
\hline UI1CMD & 0.582 & 0.69 & UP1CBL & 0.476 & 0.023 & \\
\hline UI2CBL & 1.029 & -0.099 & UP1CMD & 0.931 & -0.338 & \\
\hline UCCBL & 0.867 & -0.614 & UP2CBL & 0.494 & 0.768 & \\
\hline UCCMD & 1.08 & -0.221 & UP2CMD & 0.992 & -0.231 & \\
\hline \multirow[t]{4}{*}{ UI2CMD } & 0.934 & 0.504 & UM1CBL & 0.305 & 0.634 & \\
\hline & & & UMICMD & 0.817 & 0.05 & \\
\hline & & & UM2CBL & 0.749 & -0.04 & \\
\hline & & & UM2CMD & 0.443 & -0.115 & \\
\hline \multicolumn{7}{|c|}{ Mandibular } \\
\hline Anterior & $\begin{array}{c}\text { PC1 } \\
(75.5 \%)\end{array}$ & $\begin{array}{c}\text { PC2 } \\
(10.5 \%)\end{array}$ & Posterior & $\begin{array}{c}\text { PC1 } \\
(46.7 \%)\end{array}$ & $\begin{array}{c}\text { PC2 } \\
(19.6 \%)\end{array}$ & $\begin{array}{c}\text { PC3 } \\
(13.0 \%)\end{array}$ \\
\hline LI1CMD & 0.762 & 0.114 & LP1CBL & 0.69 & -0.176 & 0.115 \\
\hline LI2CBL & 0.883 & 0.371 & LP1CMD & 0.617 & -0.43 & -0.331 \\
\hline LI2CMD & 0.924 & -0.089 & LP2CBL & 0.676 & -0.007 & 0.028 \\
\hline LCCBL & 0.788 & 0.259 & LP2CMD & 0.75 & -0.386 & -0.309 \\
\hline \multirow[t]{4}{*}{ LCCMD } & 0.962 & -0.557 & LM1CBL & 0.539 & 0.032 & 0.569 \\
\hline & & & LM1CMD & 0.756 & 0.419 & -0.148 \\
\hline & & & LM2CBL & 0.426 & -0.079 & 0.519 \\
\hline & & & LM2CMD & 0.438 & 0.87 & -0.18 \\
\hline
\end{tabular}

* Number in bracket is the percentage variance explained by each component 
size is likely a contributing factor to the absence of any organization of the maxillary PC scores along sex or temporal lines, as the maxillary anterior and posterior PC analyses only include sixteen (pre-2000 BP $n=10$; post-2000 BP $n=6$ ) and twenty (pre-2000 BP $n=11$; post-2000 $\mathrm{BP} \mathrm{n}=9$ ) individuals, respectively, from the total odontometric sample. Variability and a lack of patterning are also evident in the principal components results for the mandibular teeth. The PC1 scores for the anterior and posterior mandibular teeth, the PC2 scores for the anterior mandibular teeth, and the PC3 scores for the posterior mandibular teeth do not produce any significant patterns. For all of these variable sets the PC1 scores are scattered across the axis, and the scores for all four sub-groups cluster together along the PC2 axis (Figures 4.14 to 4.16). A very weak pattern emerges only for the mandibular posterior PC2 scores. The pre-2000 BP male mandibular posterior PC2 scores fall at the low end and the post-2000 BP male scores fall at the high end of the axis (Figure 4.15), indicating that the pre-2000 BP males possess larger premolars, but smaller molars in the mesiodistal plane, compared to the post-2000 BP males. Small sample size continues to be a limiting factor in the PC analysis of the mandibular cervical dimensions, with mandibular anterior and posterior principal components including only seventeen (pre-2000 BP n=9; post-2000 BP $\mathrm{n}=8$ ) and twenty (pre-2000 BP $\mathrm{n}=10$; post-2000 BP $\mathrm{n}=10$ ) individuals, respectively. These results reinforce the limitations in applying principal components analysis to small datasets as specimens must possess all metric variables to be included in the analysis. As discussed in Chapter 3, when selecting cervical odontometric dimensions for analysis an effort was made to maximize the number of variables and specimens included in each PCA. As these results attest, this proved difficult for a total odontometric sample already limited in size. 
Figure 4.12. Scatterplot of PC1 and PC2 scores for Maxillary Anterior Cervical Variables

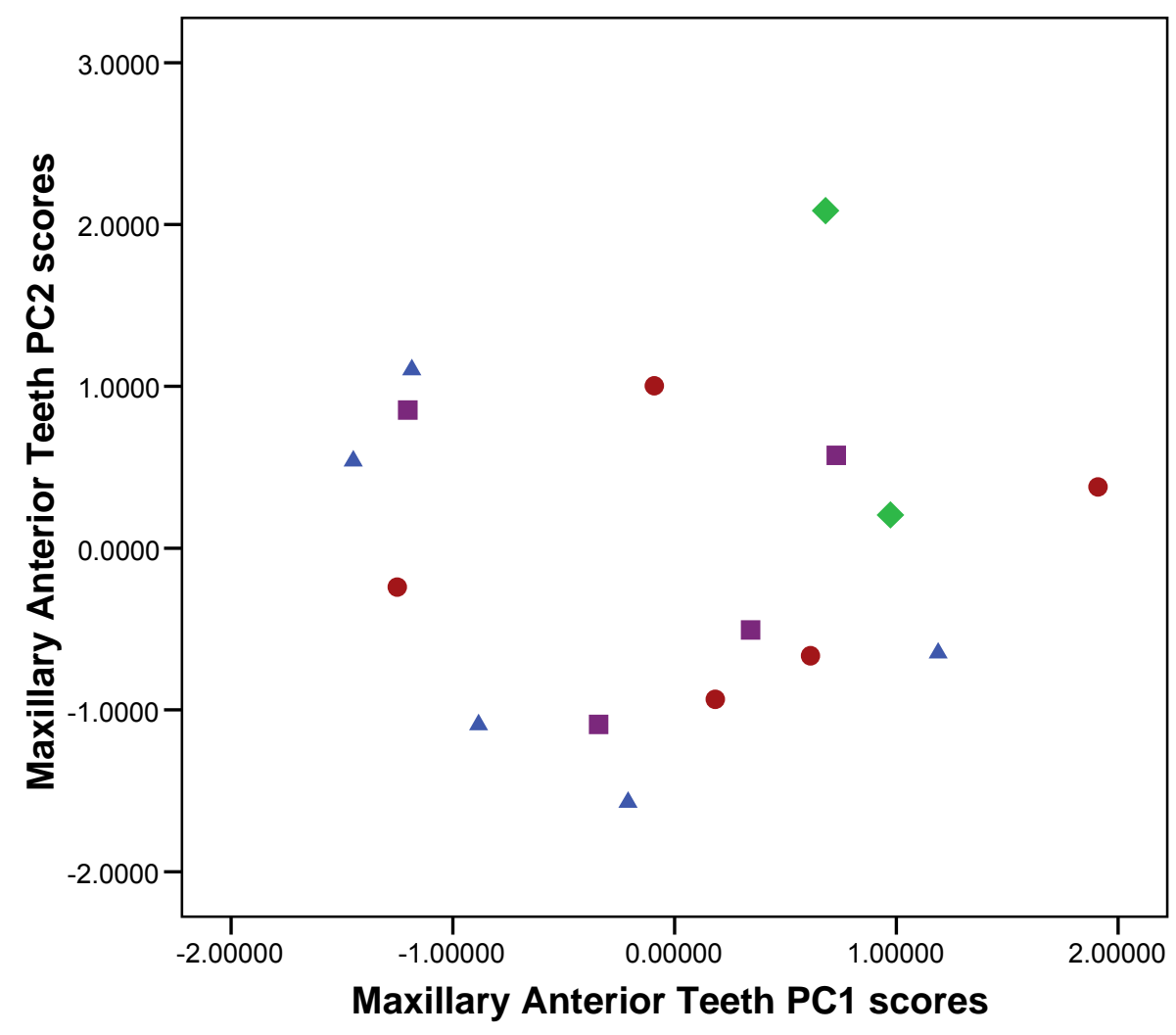

A F pre $2000 \mathrm{BP}, \mathrm{N}=5$ $\mathrm{F}$ post $2000 \mathrm{BP}, \mathrm{N}=2$ $M$ pre $2000 \mathrm{BP}, \mathrm{N}=5$

M post $2000 \mathrm{BP}, \mathrm{N}=4$

Figure 4.13. Scatterplot of PC1 and PC2 scores for Maxillary Posterior Cervical Variables

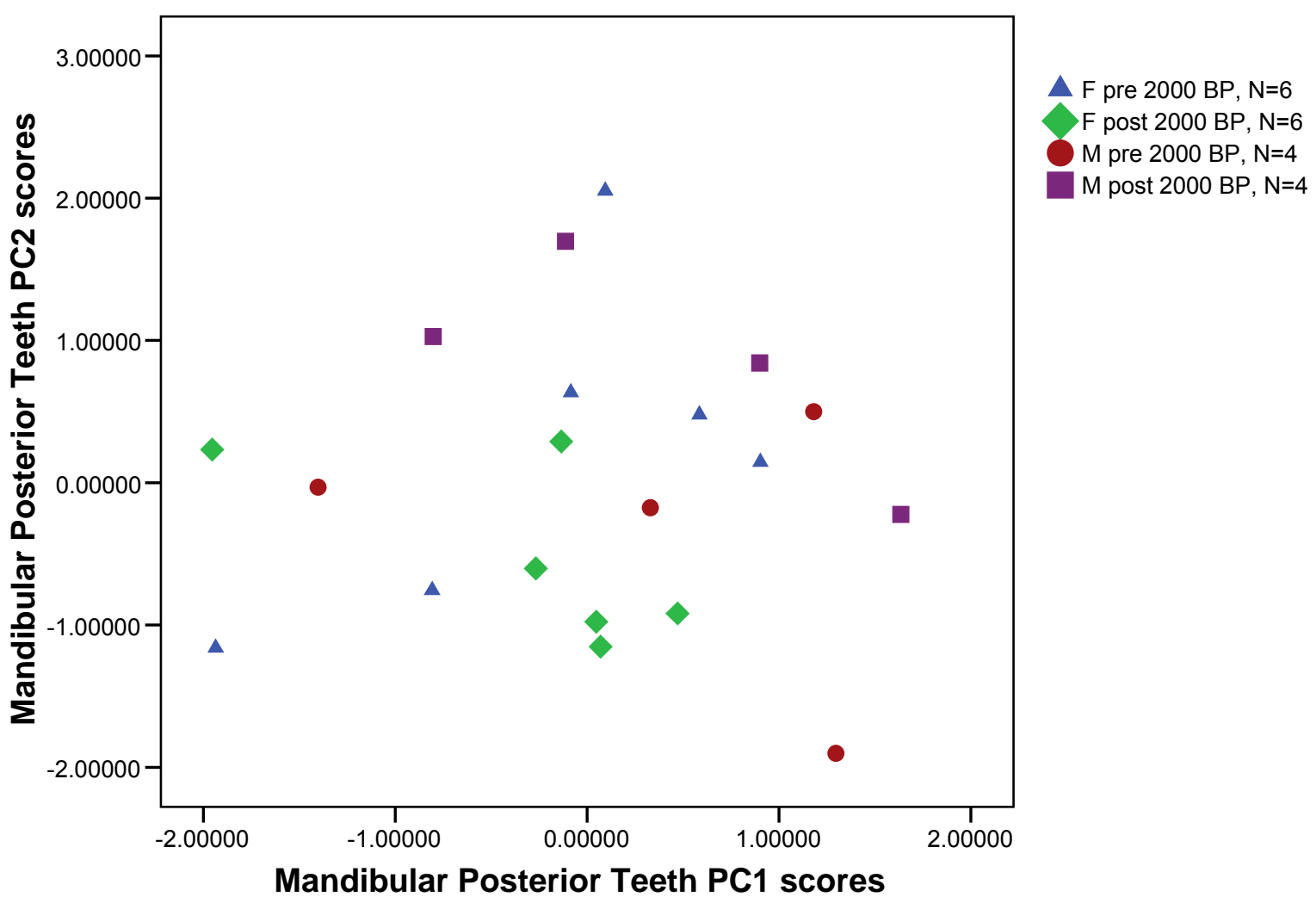


Figure 4.14. Scatterplot of PC1 and PC2 scores for Mandibular Anterior Cervical Variables

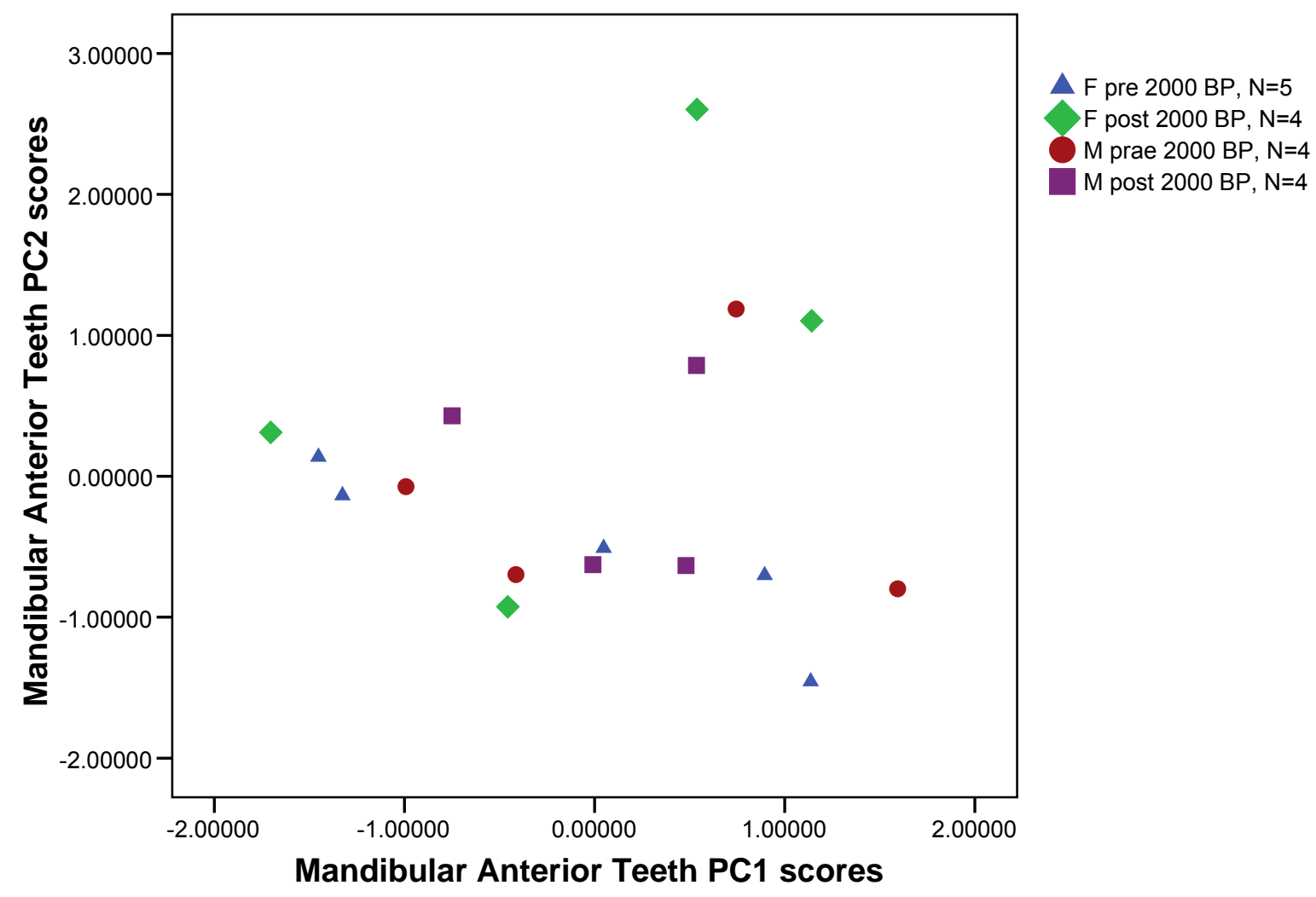

Figure 4.15. Scatterplot of PC1 and PC2 scores for Mandibular Posterior Cervical Variables

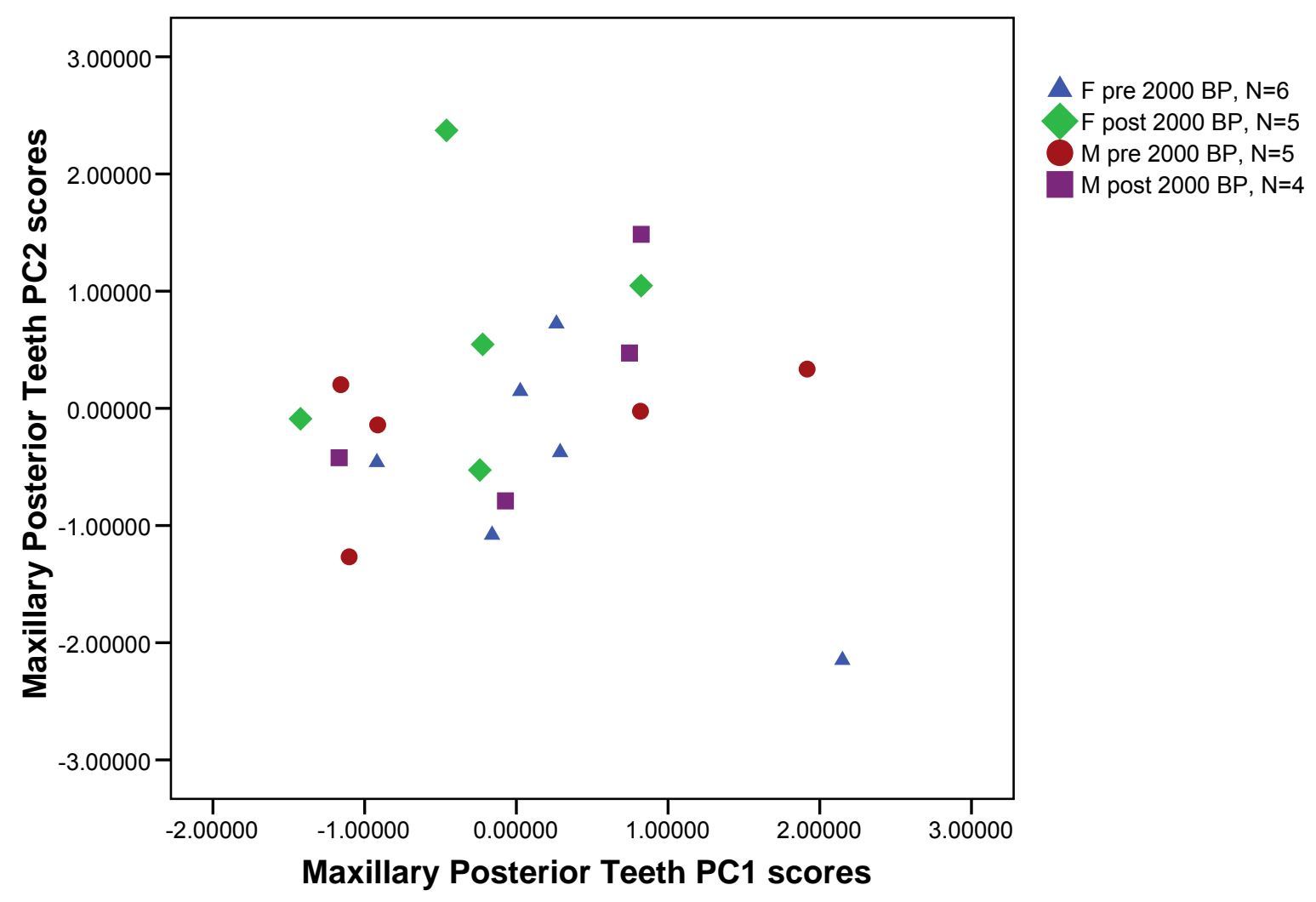


Figure 4.16. Scatterplot of PC1 and PC3 scores for Mandibular Posterior Cervical Variables

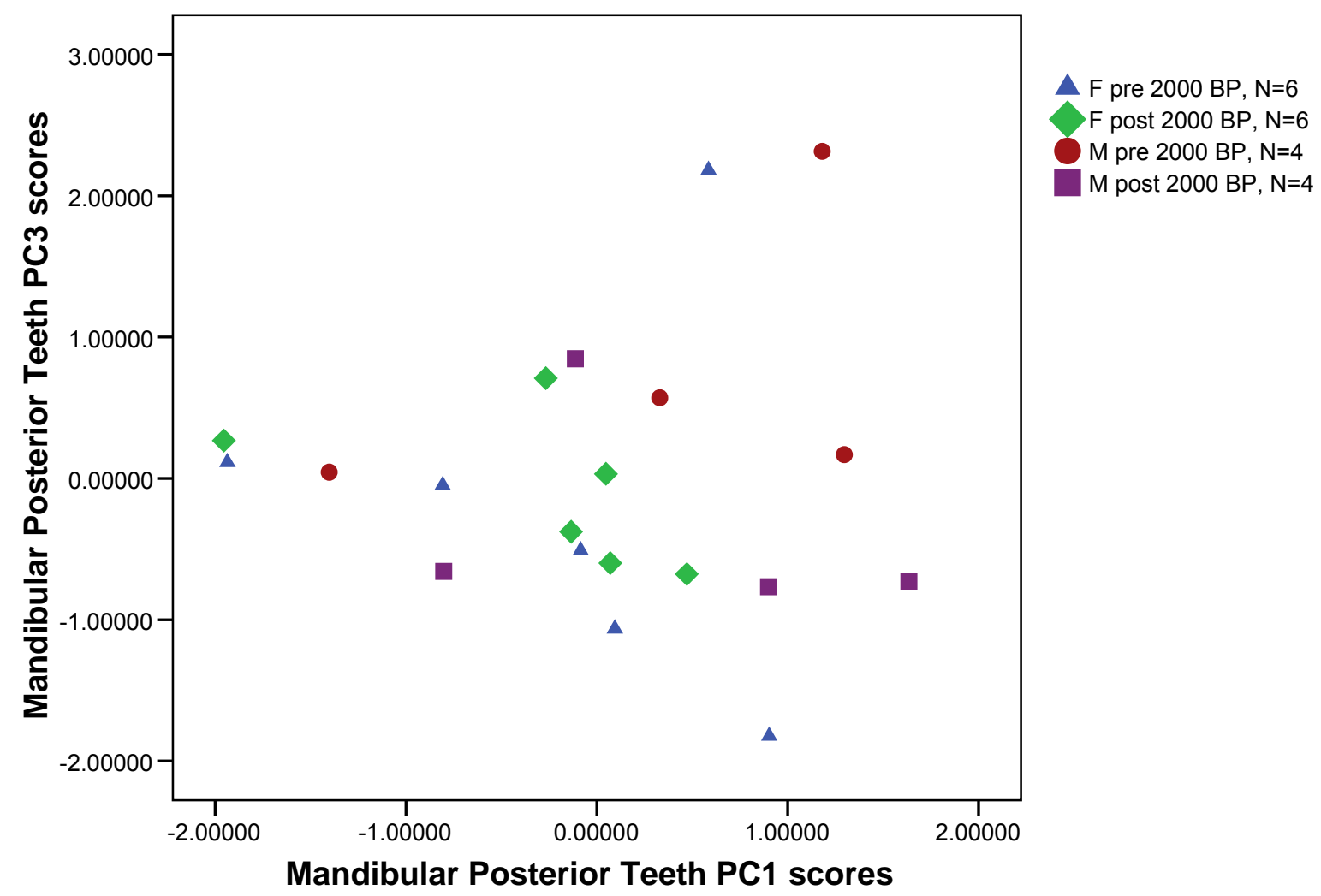

Because only a small portion of the total cervical odontometric sample is included in the principal component analysis, it is risky to extend inferences to the group as a whole. Yet, these results are consistent with the univariate findings in demonstrating that there are no significant changes in the size and shape of the dentition through time.

\subsubsection{Curve Estimation Regression of Cervical Dimensions}

Regression of the principal component scores against time reveals some changes in tooth size that were not detectable in the principal components results alone. The relationship between maxillary anterior PC1 scores and ${ }^{14} \mathrm{C}$ date is not statistically supported (females $\mathrm{r}^{2}=0.510, \mathrm{p}=0.240$; males $\mathrm{r}^{2}=0.576, \mathrm{p}=0.076$ ), yet it is visible from the scatter plot that both male and female tooth size undergoes a temporary and significant decrease 
around $3500 \mathrm{BP}$, returning to pre-3500 BP values around $2000 \mathrm{BP}$ (Figure 4.17). In

comparison, the regression of the maxillary anterior PC2 scores against ${ }^{14} \mathrm{C}$ does not point to any change in tooth shape for females $\left(r^{2}=0.497, p=0.253\right)$ or males $\left(r^{2}=0.032, p=0.908\right)$ (Figure 4.18). Dimensions of the maxillary posterior teeth, show a significant relationship between the female PC1 scores and ${ }^{14} \mathrm{C}$ date $\left(\mathrm{r}^{2}=0.657, \mathrm{p}=0.014\right)$, but not for males $\left(\mathrm{r}^{2}=\right.$ $0.054, \mathrm{p}=0.621)$ (Figure 4.19). Although not statistically supported for males, both sexes display a short lived reduction in the size of the maxillary posterior teeth between approximately 3500 to $2000 \mathrm{BP}$. Maxillary posterior tooth sizes return to pre-3500 BP levels immediately following $2000 \mathrm{BP}$. No relationship is evident in the maxillary posterior PC2 regression for either females $\left(r^{2}=0.350, p=0.179\right)$ or males $\left(r^{2}=0.024, p=0.929\right)$ (Figure 4.20).

Figure 4.17. Scatterplot of regression scores for maxillary anterior cervical variables: PC1 vs. ${ }^{14} \mathrm{C}$. Females $=$ dashed line, males $=$ solid line.

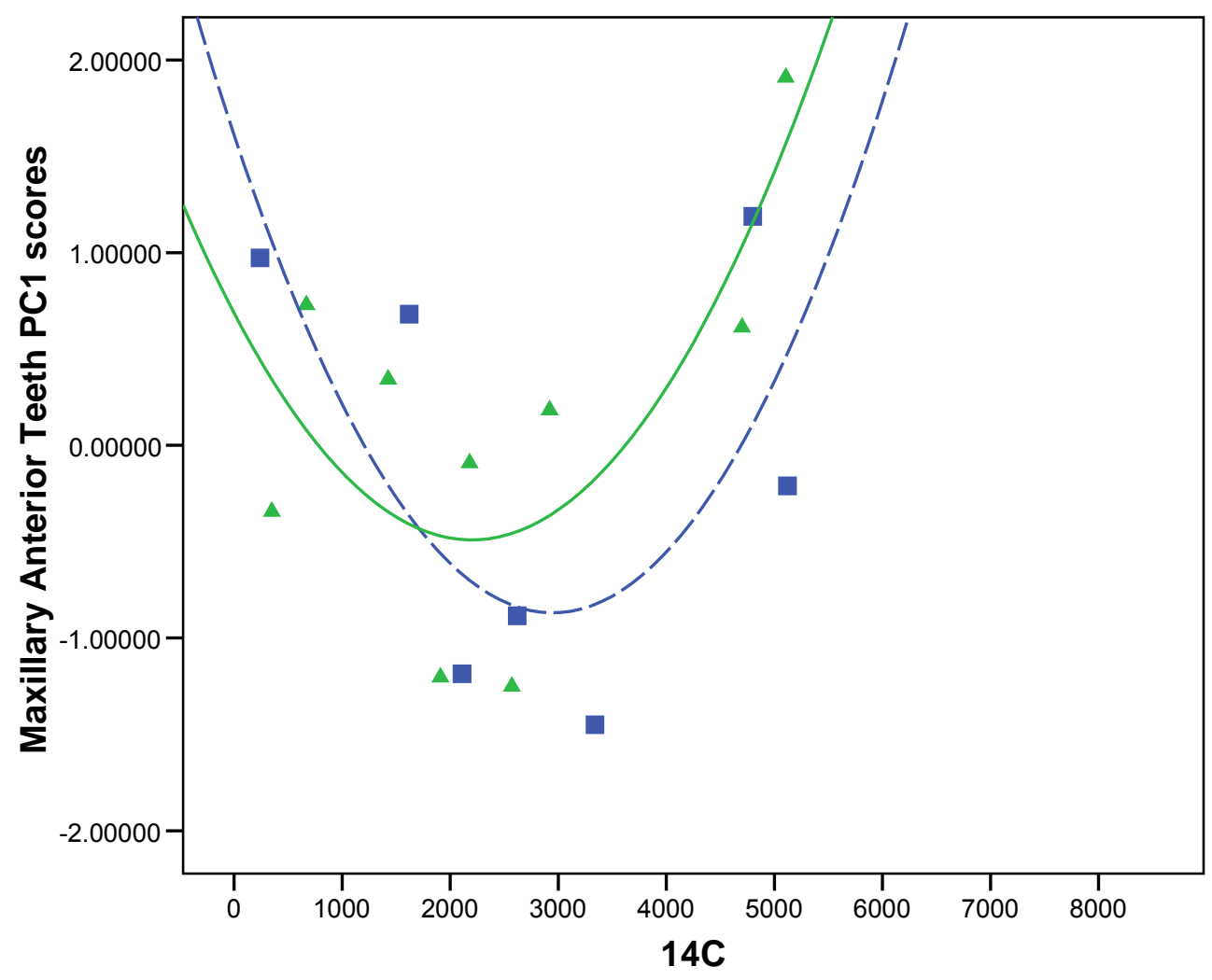


Figure 4.18. Scatterplot of regression scores for maxillary anterior cervical variables: PC2 vs. ${ }^{14} \mathrm{C}$. Females $=$ dashed line, males $=$ solid line.

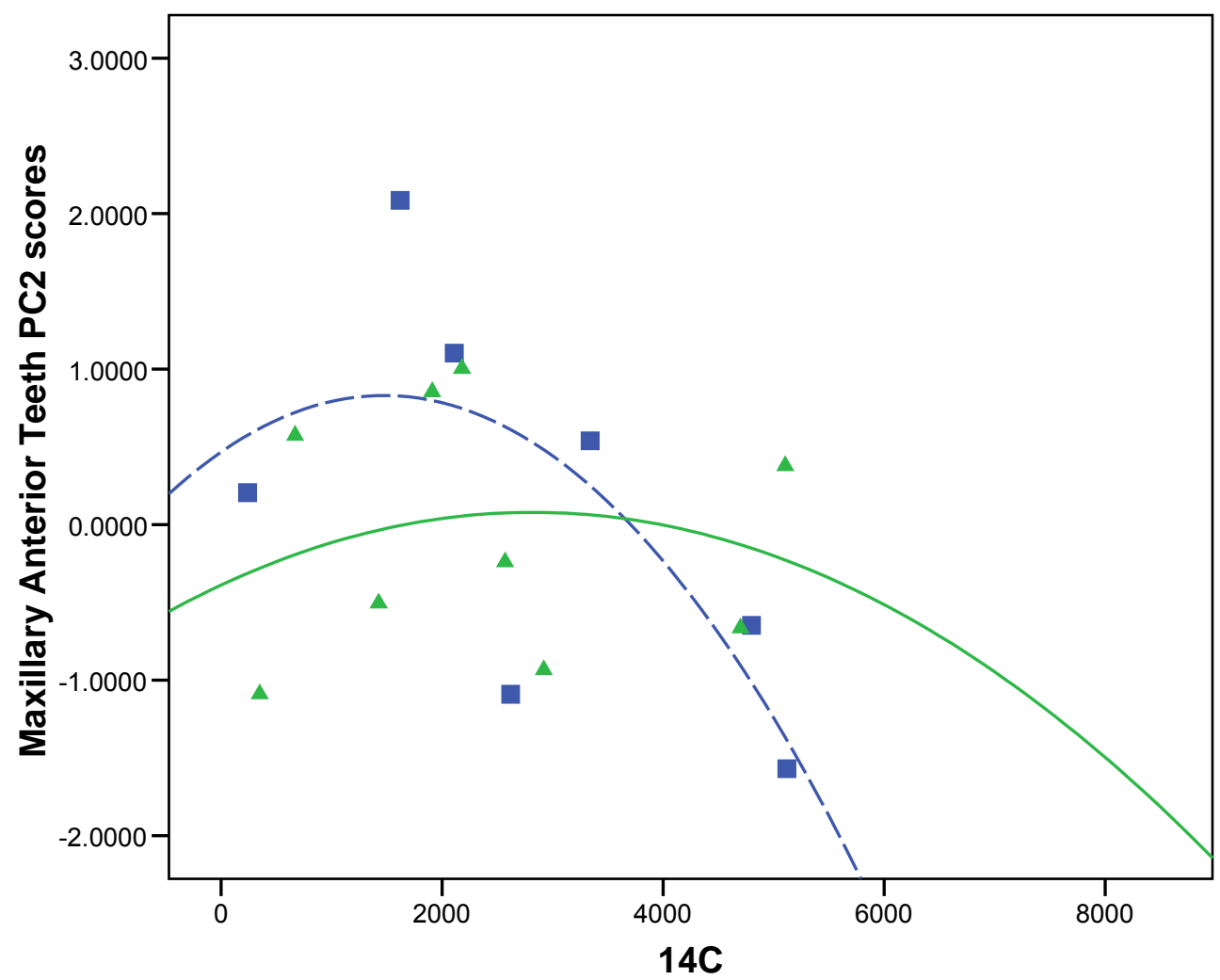

R Sq Quadratic $=0.497$ R Sq Quadratic $=0.032$

Figure 4.19. Scatterplot of regression scores for maxillary posterior cervical variables: PC1 vs. ${ }^{14} \mathrm{C}$. Females $=$ dashed line, males $=$ solid line

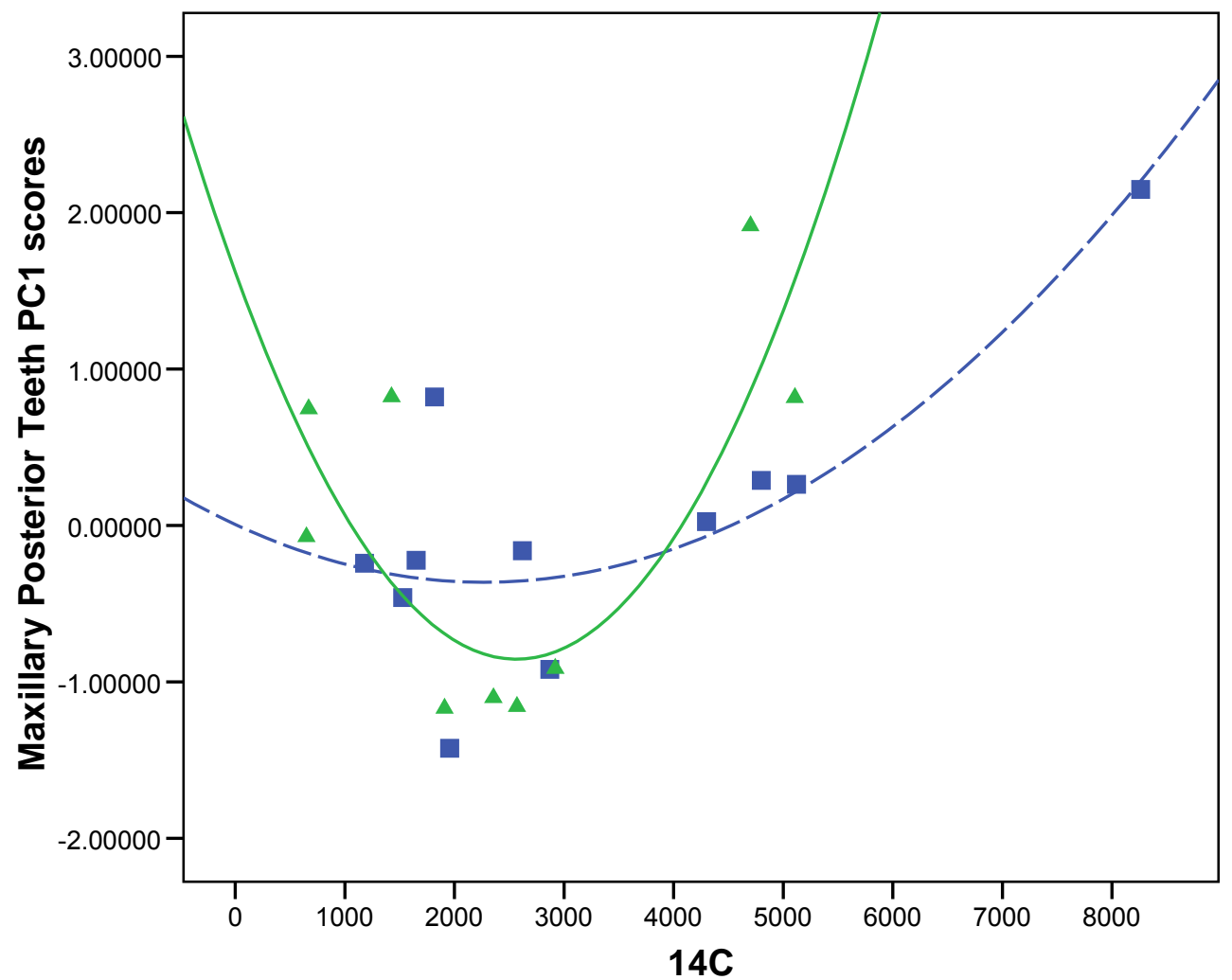

R Sq Quadratic $=0.657$ R Sq Quadratic $=0.621$ 
Figure 4.20. Scatterplot of regression scores for maxillary posterior cervical variables: PC2 vs. ${ }^{14} \mathrm{C}$. Females $=$ dashed line, males $=$ solid line

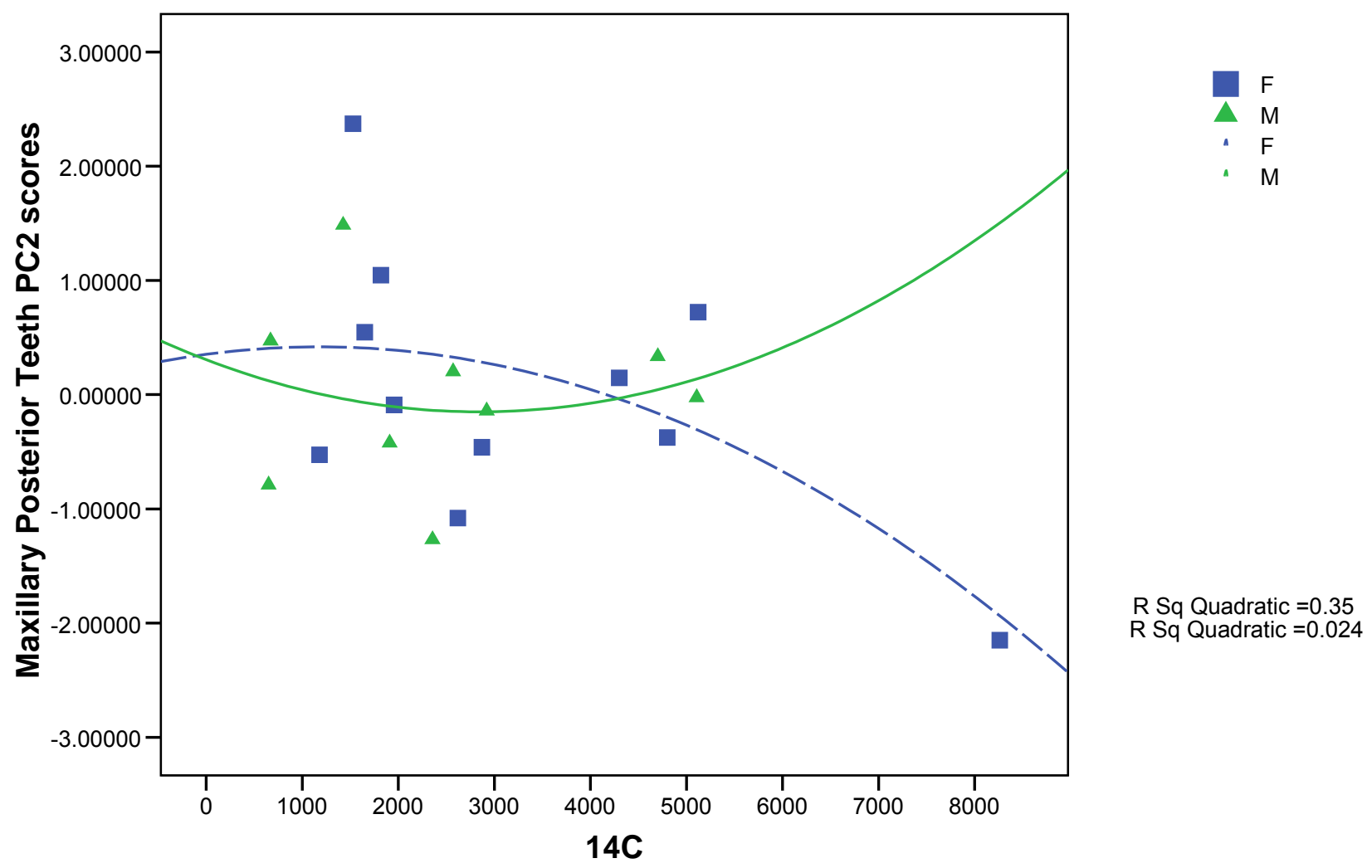

The drop in PC1 scores between 2000 BP and 3000 BP with a recovery in size to pre$3000 \mathrm{BP}$ values occurs in all of the mandibular regression analyses, although all are not statistically significant. A decrease in mandibular anterior tooth size is evident (Figure 4.21), but this relationship is not statistically supported for females $\left(r^{2}=0.594, p=0.067\right)$ or males $\left(r^{2}=0.406, p=0.272\right)$. Statistically significant relationships are not supported between mandibular anterior tooth shape and time for females $\left(r^{2}=0.367, p=0.253\right)$ or males $\left(r^{2}=\right.$ $0.327, \mathrm{p}=0.372)($ Figure 4.22$)$. In contrast, the regression against date indicates that significant temporal changes took place in the size of the male mandibular posterior teeth between approximately 3500 to $2000 \mathrm{BP}\left(\mathrm{r}^{2}=0.702, \mathrm{p}=0.048\right)$ (Figure 4.23). A reduction in female tooth size occurs around the same time, but this is not significant $\left(\mathrm{r}^{2}=0.248, \mathrm{p}=\right.$ 0.278). As with the maxillary anterior teeth, the absence of any patterns in the regression 
Figure 4.21. Scatterplot of regression scores for mandibular anterior cervical variables: PC1 vs. ${ }^{14} \mathrm{C}$. Females $=$ dashed line, males $=$ solid line

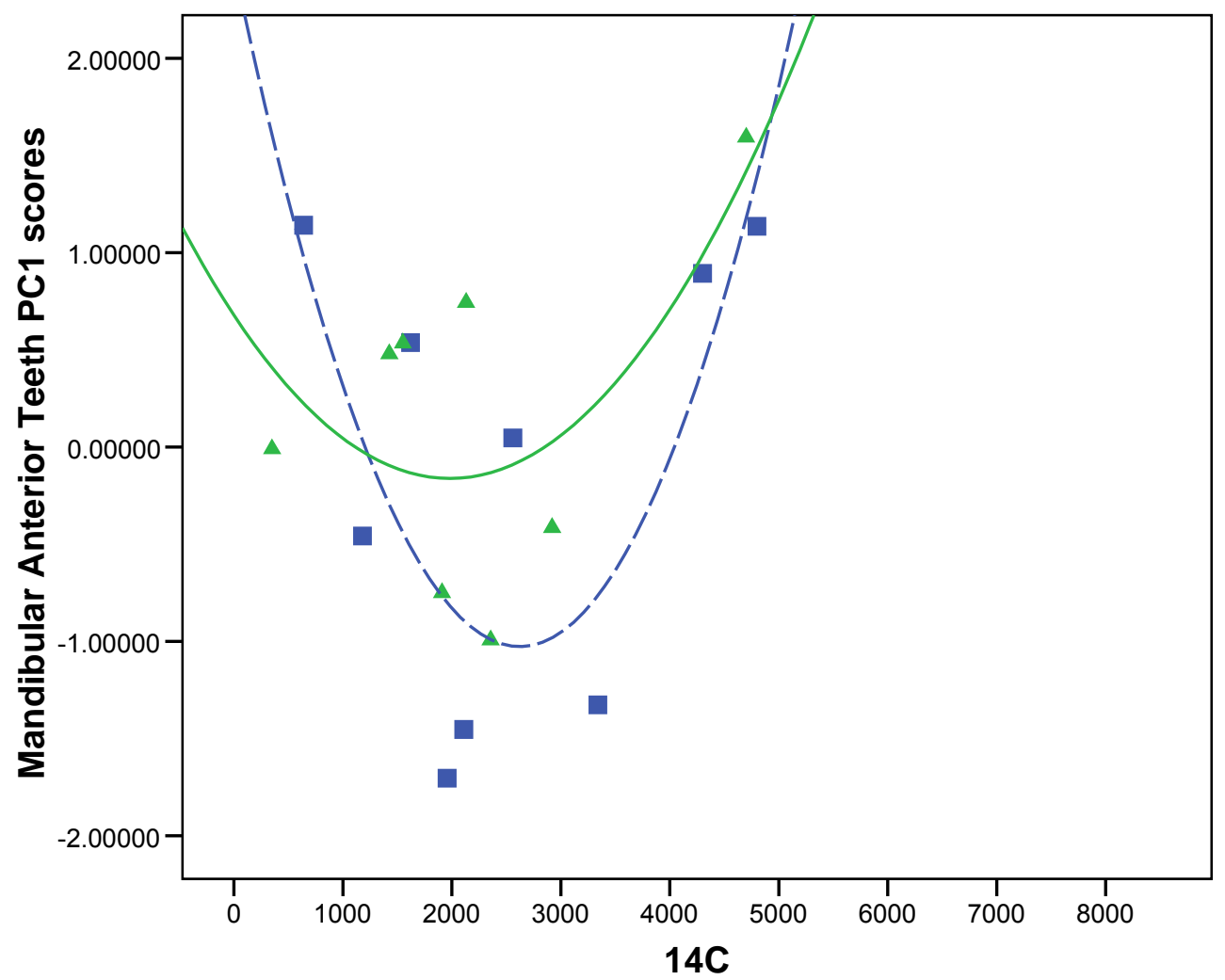

R Sq Quadratic $=0.594$

Figure 4.22. Scatterplot of regression scores for mandibular anterior cervical variables: PC2 vs. ${ }^{14} \mathrm{C}$. Females $=$ dashed line, males $=$ solid line

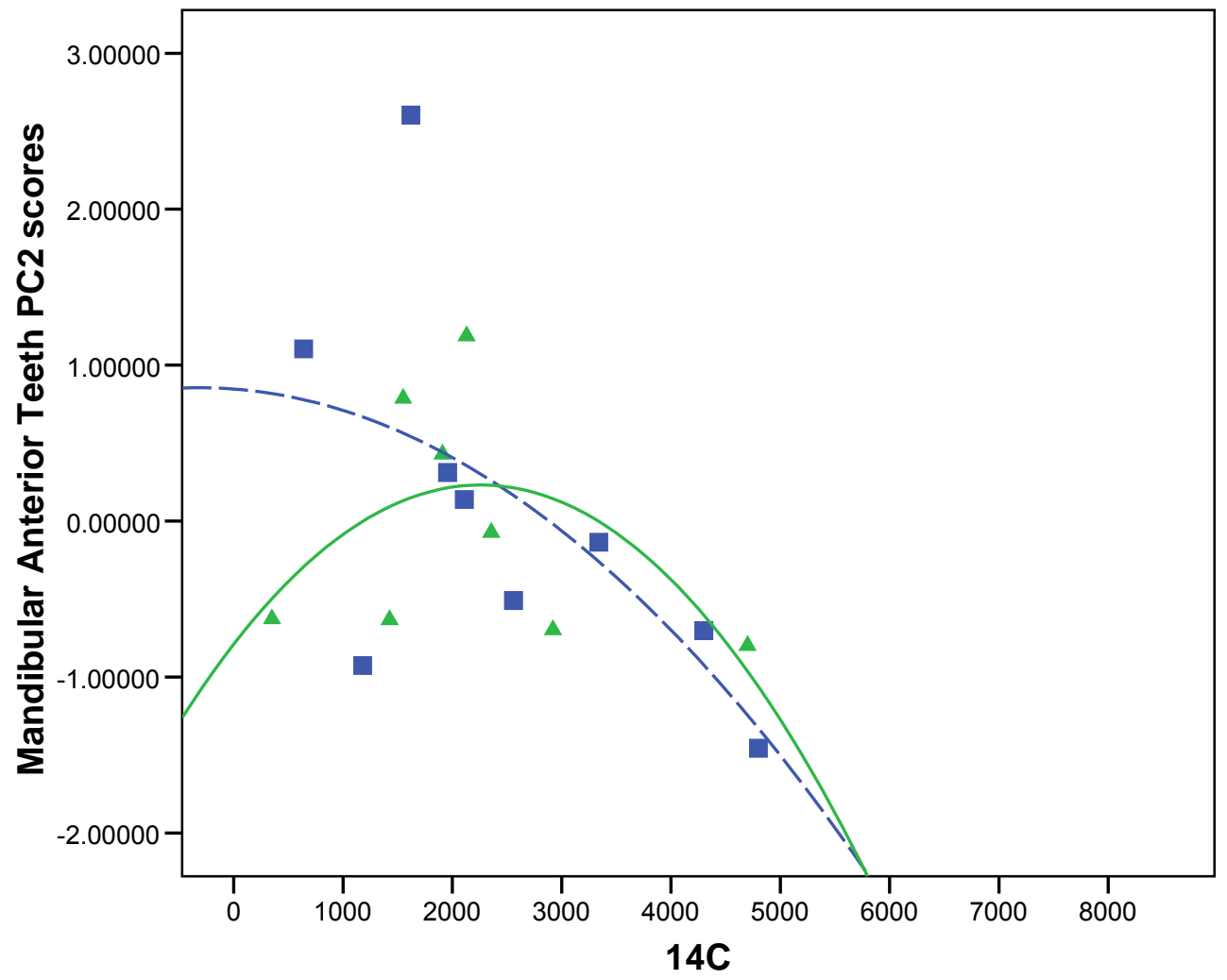


Figure 4.23. Scatterplot of regression scores for mandibular posterior cervical variables:

PC1 vs. ${ }^{14} \mathrm{C}$. Females $=$ dashed line, males $=$ solid line.

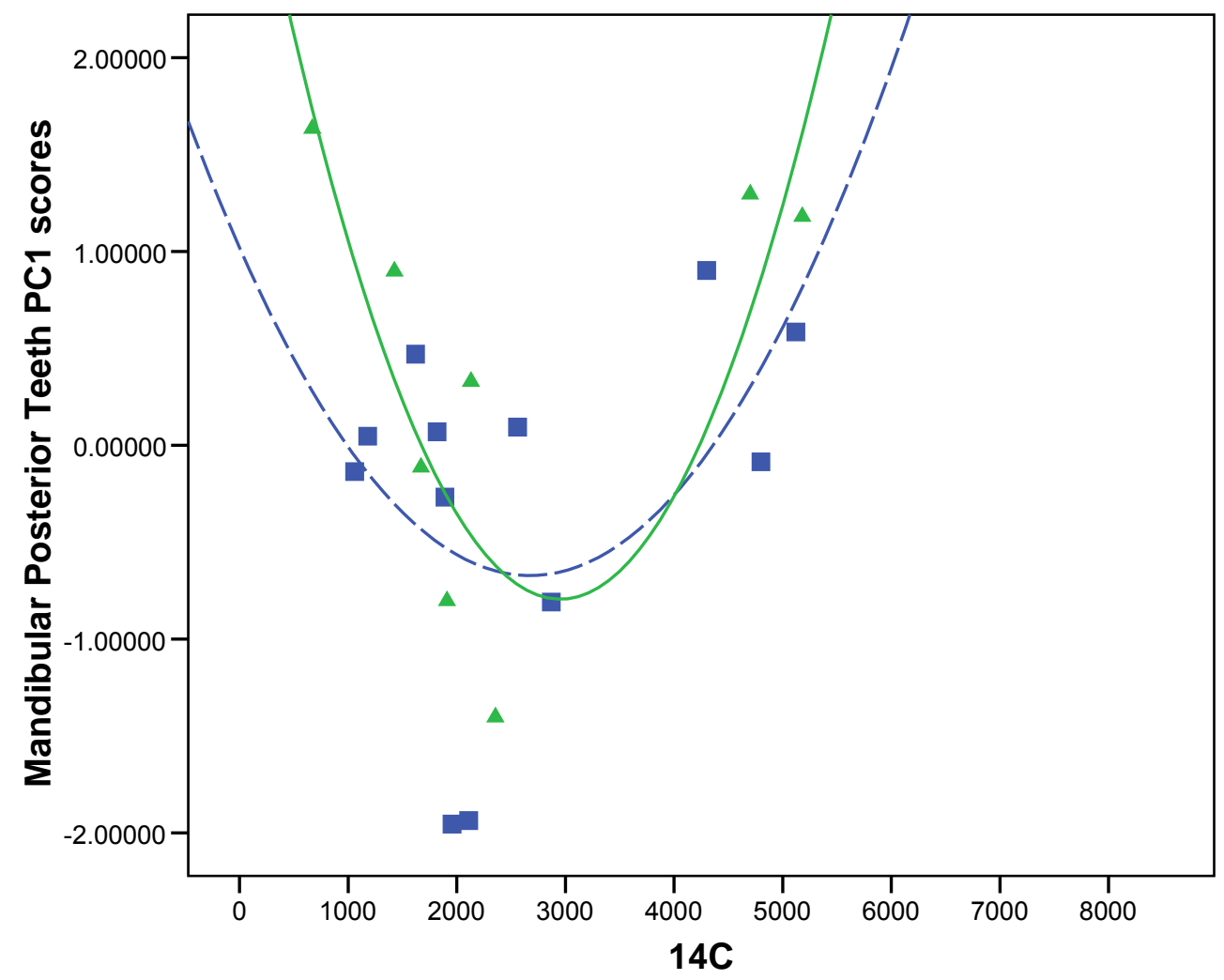

R Sq Quadratic $=0.248$

R Sq Quadratic $=0.702$

Figure 4.24. Scatterplot of regression scores for mandibular posterior cervical variables:

$\mathrm{PC} 2$ vs. ${ }^{14} \mathrm{C}$. Females $=$ dashed line, males $=$ solid line.

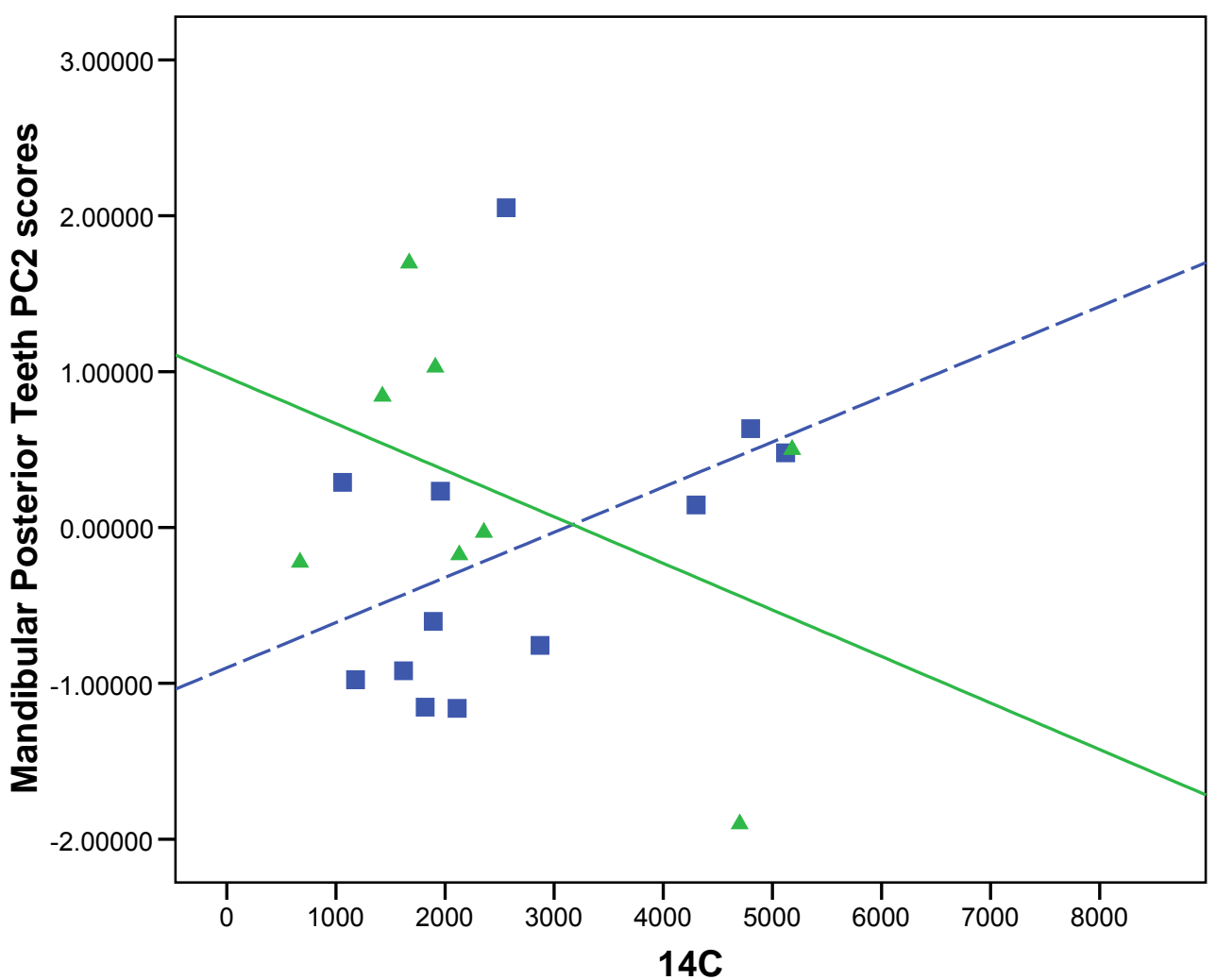


Figure 4.25. Scatterplot of regression scores for mandibular posterior cervical variables: PC3 vs. ${ }^{14} \mathrm{C}$. Females $=$ dashed line, males $=$ solid line.

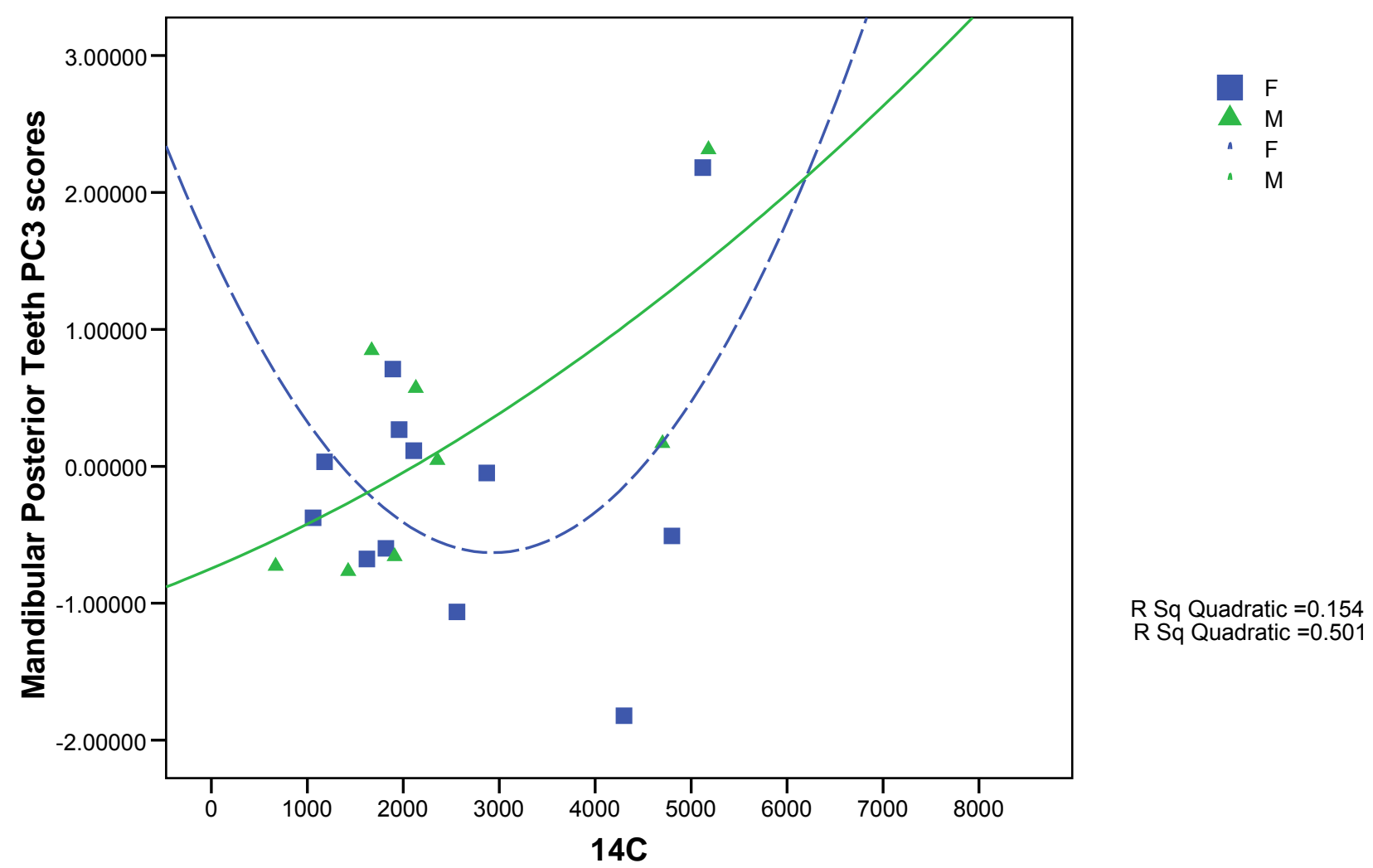

analyses of the mandibular posterior PC2 and PC 3 scores suggests that no significant changes in female $\left(\mathrm{PC} 2 \mathrm{r}^{2}=0.176, \mathrm{p}=0.175 ; \mathrm{PC} 3 \mathrm{r}^{2}=0.154, \mathrm{p}=0.470\right)$ or male $\left(\mathrm{PC} 2 \mathrm{r}^{2}=\right.$ $0.193, \mathrm{p}=0.277 ;$ PC3 $\left.\mathrm{r}^{2}=0.499, \mathrm{p}=0.050\right)$ tooth morphology occurred through time (Figures 4.24 and 4.25).

\subsubsection{Comparison with Other South African Samples}

Comparative odontometric data are available only for the maximum mesiodistal and buccolingual variables. The small size of the Eastern Cape maximum odontometric sample precludes division into sub-groupings by sex and date. Instead the total Eastern Cape maximum odontometric sample is compared to published data. Four sources of published 
odontometric summary data were used: prehistoric LSA from South Africa (Pfeiffer in press), early Griqua from South Africa (Kieser 1985), modern South African Bantu-speakers (skeletal) (Kieser et al. 1987), and San (Bushmen, skeletal) (Drennan 1929). T-tests using means and standard deviations were employed to compare MD and BL diameters of the maxillary and mandibular first and second molars.

Significant differences were not identified between the pooled Eastern Cape and LSA samples (Table 4.8). The Eastern Cape mean values are larger than the Griqua means for all of the odontometric variables, yet only four statistically significant results were produced for females (UM1 BL, UM2 BL, LM2 BL and MD) and five significant results for males (UM1 BL, UM2 BL, LM1 BL and MD, LM2 BL). The greatest number of significant differences for females occurs between the Eastern Cape and South African Bantu-speaking skeletal samples where all but one (LM2 MD) of the maximum odontometric variables yields a significant result. For the males, only four of the eight variables yield significant differences. In general the mean Bantu-speaking odontometric values for both sexes are larger than the corresponding Eastern Cape means. Comparisons with Drennan's male San data produces four significant differences (UM1 BL, LM1 MD, LM2 BL and MD), yet the Eastern Cape means are larger than the San for all variables. The absence of significant results between the Eastern Cape and LSA data, as well as the prevalence of significant differences between the Eastern Cape and Bantu-speaking values, support the idea of genetic continuity in the Eastern Cape sample through time. 


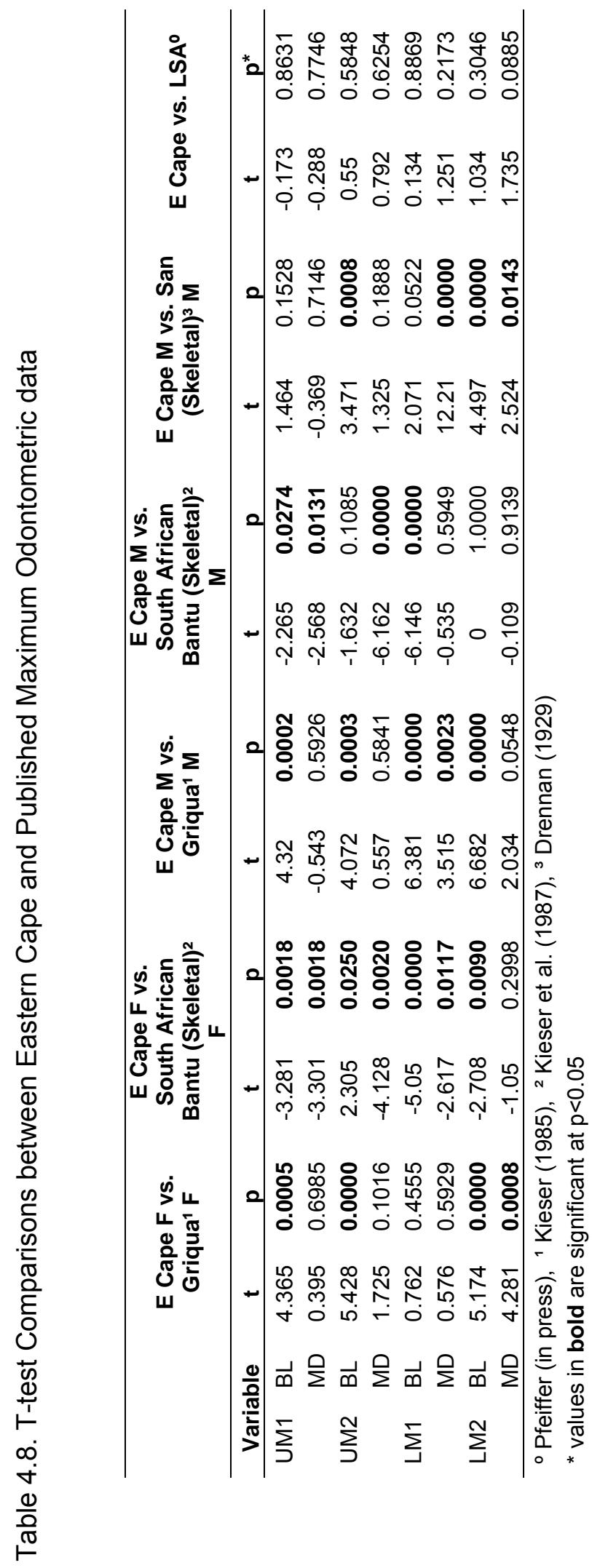




\subsection{Postcranial Measurements}

As described in Chapter 3, metric variables that quantify various dimensions (length, breadth, diameter, and circumference) of the postcranial skeleton were collected for all available elements. A total of 56 individuals were available for the postcranial metric analysis.

\subsubsection{Univariate Analysis}

One-way analysis of variance (ANOVA) was employed to explore potential differences between the postcranial variable means. Variables yielding significant results were subjected to post-hoc tests to determine which of the four sub-groups differed. The results of the one-way ANOVAs indicate that some changes in overall body size occurred in the Eastern Cape through time. The greatest number of statistically significant differences (34 variables) occur between the pre-2000 BP females and the post-2000 BP (34 variables) and pre-2000 BP (25 variables) males (Table 4.9). The results indicate that the post-2000 BP males are larger than the pre-2000 BP females throughout the postcranial skeleton, while size differences between post-2000 males and females are limited more to the lower limb. Considerably fewer variables differ significantly between post $2000 \mathrm{BP}$ females and the two male sub-groups (pre-2000 BP $n=12$; post-2000 BP $n=15$ ). The distribution of variables with significantly different mean values suggests that size differences between post-2000 females and males are concentrated in the lower limbs. These sex differences may be attributable to sexual dimorphism, yet the magnitude and number of differences also suggest that some true changes in body size may have occurred. Within-sex comparisons did not produce significant temporal differences among the female and male sub-groups. Post-2000 BP females are significantly larger than pre-2000 BP females in only five variables that are 
Table 4.9. Probability values for Postcranial Metric Post-hoc1 ANOVA tests - variables that differ significantly between sub-groups $(p<0.05)$

\begin{tabular}{|c|c|c|c|c|c|c|}
\hline \multirow{2}{*}{$\begin{array}{l}\text { Postcranial } \\
\text { Metric } \\
\text { Variables }\end{array}$} & \multicolumn{3}{|c|}{ Pre 2000 BP Females } & \multicolumn{2}{|c|}{ Post 2000 BP Females } & \multirow{2}{*}{$\begin{array}{c}\text { Pre } 2000 \text { BP Males } \\
\text { vs. } \\
\text { Post } 2000 \text { BP } \\
\text { Males }\end{array}$} \\
\hline & $\begin{array}{c}\text { vs. } \\
\text { Post } 2000 \text { BP } \\
\text { Males }\end{array}$ & $\begin{array}{c}\text { vs. } \\
\text { Post } 2000 \text { BP } \\
\text { Females }\end{array}$ & $\begin{array}{c}\text { vs. } \\
\text { Pre } 2000 \text { BP } \\
\text { Males }\end{array}$ & $\begin{array}{c}\text { vs. } \\
\text { Post } 2000 \text { BP } \\
\text { Males }\end{array}$ & $\begin{array}{c}\text { vs. } \\
\text { Pre } 2000 \text { BP } \\
\text { Males }\end{array}$ & \\
\hline CLAVXL & 0.002 & $\mathrm{x}$ & 0.019 & $\mathrm{x}$ & $\mathrm{x}$ & $\mathrm{x}$ \\
\hline CLAVAPD $^{2}$ & $\mathrm{x}$ & $x$ & 0.000 & $x$ & $x$ & $x$ \\
\hline CLAVSID & 0.037 & $x$ & $\mathrm{x}$ & $\mathrm{x}$ & $x$ & $\mathrm{x}$ \\
\hline SCAPXBR & 0.000 & $\mathrm{x}$ & $\mathrm{x}$ & 0.009 & $x$ & 0.013 \\
\hline SCAPSPIL & 0.020 & $x$ & $x$ & $\mathrm{x}$ & $x$ & $\mathrm{x}$ \\
\hline SCAPGLBR & 0.023 & $x$ & $x$ & $x$ & $x$ & $x$ \\
\hline SCAPGLHT & $\mathrm{x}$ & $x$ & 0.018 & $x$ & $x$ & $x$ \\
\hline HUMIDXD² & $x$ & 0.037 & $x$ & $x$ & $x$ & $x$ \\
\hline HUMHEADD & $x$ & $x$ & 0.000 & $x$ & 0.010 & $x$ \\
\hline HUMEPBR & 0.005 & $x$ & 0.005 & $x$ & $x$ & $x$ \\
\hline RADXL & 0.021 & $x$ & 0.022 & $x$ & $x$ & $x$ \\
\hline RADHEADD $^{2}$ & 0.005 & $x$ & 0.001 & $x$ & $x$ & $x$ \\
\hline RADAPD & 0.029 & $x$ & $\mathrm{x}$ & $x$ & $x$ & $x$ \\
\hline ULNALX & 0.048 & $x$ & $x$ & $x$ & $x$ & $x$ \\
\hline SACRINDX & $x$ & $x$ & 0.029 & $x$ & $x$ & $x$ \\
\hline INNOHT & 0.038 & $x$ & $x$ & $x$ & $x$ & $x$ \\
\hline ILIACBR & 0.011 & $x$ & $x$ & $x$ & 0.016 & $x$ \\
\hline PUBISLN & $x$ & $x$ & $x$ & $x$ & 0.020 & $x$ \\
\hline BIBBR & 0.017 & 0.042 & $x$ & $\mathrm{x}$ & $\mathrm{x}$ & $x$ \\
\hline ISCPUBINDX & $\mathrm{x}$ & $x$ & $x$ & 0.002 & 0.000 & $x$ \\
\hline FEMXL & 0.018 & $x$ & $x$ & $\mathrm{x}$ & $\mathrm{x}$ & $x$ \\
\hline FEMBL & 0.043 & $x$ & $x$ & $x$ & $x$ & $\mathrm{x}$ \\
\hline FEMSTAPD & 0.001 & $x$ & 0.010 & $x$ & $x$ & $x$ \\
\hline FEMSTMLD & 0.027 & $x$ & $\mathrm{x}$ & $x$ & $x$ & $x$ \\
\hline FEMMIDADL & 0.000 & $x$ & 0.002 & 0.014 & $x$ & $x$ \\
\hline FEMVHEDD & 0.000 & $x$ & 0.018 & 0.000 & $x$ & $x$ \\
\hline FEMHHEDD & 0.000 & $x$ & 0.003 & 0.000 & $x$ & $x$ \\
\hline FEMLCAPD & 0.000 & 0.003 & $\mathrm{x}$ & 0.001 & 0.005 & $x$ \\
\hline FEMMCAPD & 0.001 & $x$ & 0.001 & 0.037 & $x$ & $x$ \\
\hline FEMEPICB & 0.000 & $x$ & 0.000 & 0.000 & 0.001 & $x$ \\
\hline FEMMIDCR & 0.000 & $x$ & 0.000 & 0.003 & 0.048 & $x$ \\
\hline TIBXL & 0.014 & $x$ & 0.029 & $\mathrm{x}$ & $\mathrm{x}$ & $x$ \\
\hline TIBEPBR & 0.000 & $x$ & 0.002 & 0.000 & 0.010 & $x$ \\
\hline TIBDEPBR & 0.000 & $x$ & 0.003 & 0.008 & & $x$ \\
\hline TIBAPNFD & 0.000 & $x$ & 0.000 & 0.000 & 0.000 & $x$ \\
\hline TIBNUTFC & 0.001 & $x$ & 0.001 & 0.000 & 0.000 & $x$ \\
\hline FIBXL & 0.027 & 0.042 & $\mathrm{x}$ & $\mathrm{x}$ & $x$ & $x$ \\
\hline FIBMSXD & 0.005 & 0.002 & 0.010 & $x$ & $x$ & $x$ \\
\hline CALCXLN & 0.000 & $\mathrm{x}$ & 0.042 & 0.013 & $x$ & $x$ \\
\hline CALCMBR & 0.006 & $x$ & 0.003 & 0.016 & 0.009 & $x$ \\
\hline $\mathrm{MT1AP}^{2}$ & $x$ & $x$ & 0.018 & $x$ & $x$ & $x$ \\
\hline MT1ML & 0.000 & $x$ & 0.023 & $x$ & 0.013 & $\mathrm{x}$ \\
\hline
\end{tabular}

${ }^{1}$ GT-2 post-hoc tests used except were indicated.

${ }^{2}$ Games-Howell post hoc test used when variance between sub-groups is unequal. 
distributed throughout the postcranial skeleton. While post-2000 BP males are larger than pre-2000 BP males for most postcranial variables, differences are significant only for scapular breadth. These negligible differences in postcranial size are of a magnitude that is consistent with growth responses to social and subsistence changes within a single population. One would expect that a greater number of postcranial variables would exhibit significant within-sex size differences if a genetically distinct migrant population existed.

\subsubsection{Multivariate Analysis}

In order to satisfy the requirements of the multivariate analyses, the postcranial metric dataset was divided into variable sets that represent size and shape dimensions of the trunk and upper and lower limbs. Variable sets were selected to accurately represent each postcranial region while maximizing the number of individuals included in each analysis. The multivariate analyses comprise 45 individuals.

\subsubsection{Principal Components Analysis}

The trunk, upper and lower limb variable sets all produced two principal components representing size (PC1) and shape (PC2) (Table 4.10). A weak temporal trend is evident in the trunk PC results, but this pattern does extend to the entire postcranial skeleton. The trunk PC1 scores for the pre-2000 BP individuals fall at the lower end of the axis, while the PC1 scores for the post-2000 BP individuals overlap at the higher end (Figure 4.26). These results further corroborate the univariate findings suggesting that there is an increase in trunk size through time in both sexes. The second principal component did not produce any meaningful trends pertaining to trunk shape. The scores for all four sub-groups overlap and span along the entire axis signifying homogeneity in trunk morphology through time. 
Table 4.10. Postcranial metric variable sets and corresponding Principal Component loadings

\begin{tabular}{lcclccccc}
\hline Trunk & $\begin{array}{c}\text { PC1 } \\
(77.0 \%)^{*}\end{array}$ & $\begin{array}{c}\text { PC2 } \\
(12.5 \%)\end{array}$ & Upper Limb & $\begin{array}{c}\text { PC1 } \\
(60.9 \%)\end{array}$ & $\begin{array}{c}\text { PC2 } \\
(14.5 \%)\end{array}$ & Lower Limb & $\begin{array}{c}\text { PC1 } \\
(58.0 \%)\end{array}$ & $\begin{array}{c}\text { PC2 } \\
(14.7 \%)\end{array}$ \\
\hline CLAVXL & 0.585 & 0.622 & HUMXL & 0.924 & -0.32 & FEMXL & 0.719 & -0.581 \\
SACRANTB & 0.941 & -0.293 & HUMMIDXD & 0.823 & 0.368 & FEMMIDADL & 0.5 & 0.441 \\
ILIACBR & 0.941 & 0.011 & HUMHEADD & 0.732 & 0.324 & FEMHHEDD & 0.815 & 0.219 \\
BIILIACBR & 0.938 & -0.106 & HUMEPBR & 0.813 & -0.054 & FEMEPICB & 0.892 & 0.104 \\
& & & RADXL & 0.818 & -0.402 & TIBXL & 0.742 & -0.591 \\
& & & RADHEADD & 0.821 & -0.18 & TIBPEPBR & 0.924 & -0.029 \\
& & & RADAPD & 0.403 & 0.687 & TIBDEPBR & 0.727 & 0.292 \\
& & & & & TIBAPNFD & 0.555 & 0.322 \\
\hline
\end{tabular}

${ }^{*}$ Number in bracket is the percentage variance explained by each component

Although the upper limb principal component analysis includes a greater number of individuals than the trunk PC analysis no temporal trend is evident in the data (Figure 4.27). Post-2000 BP females are restricted to the positive end of the PC1 axis, yet a significant portion of the scores for the other three sub-groups also fall in this range. No distinction is evident among the PC2 scores where all four sub-groups overlap considerably, signifying an absence of significant sex or temporal differences in upper limb shape.

There is even greater overlap among all sub-groups for the lower limb size (PC1) and shape (PC2) components (Figure 4.28). The PC1 and PC2 scores for the four sub-groups cluster tightly together and include the same range of values, signifying that changes in lower limb size or shape did not take place in this region across time. 
4.26. Scatterplot of PC1 and PC2 scores for the trunk variables

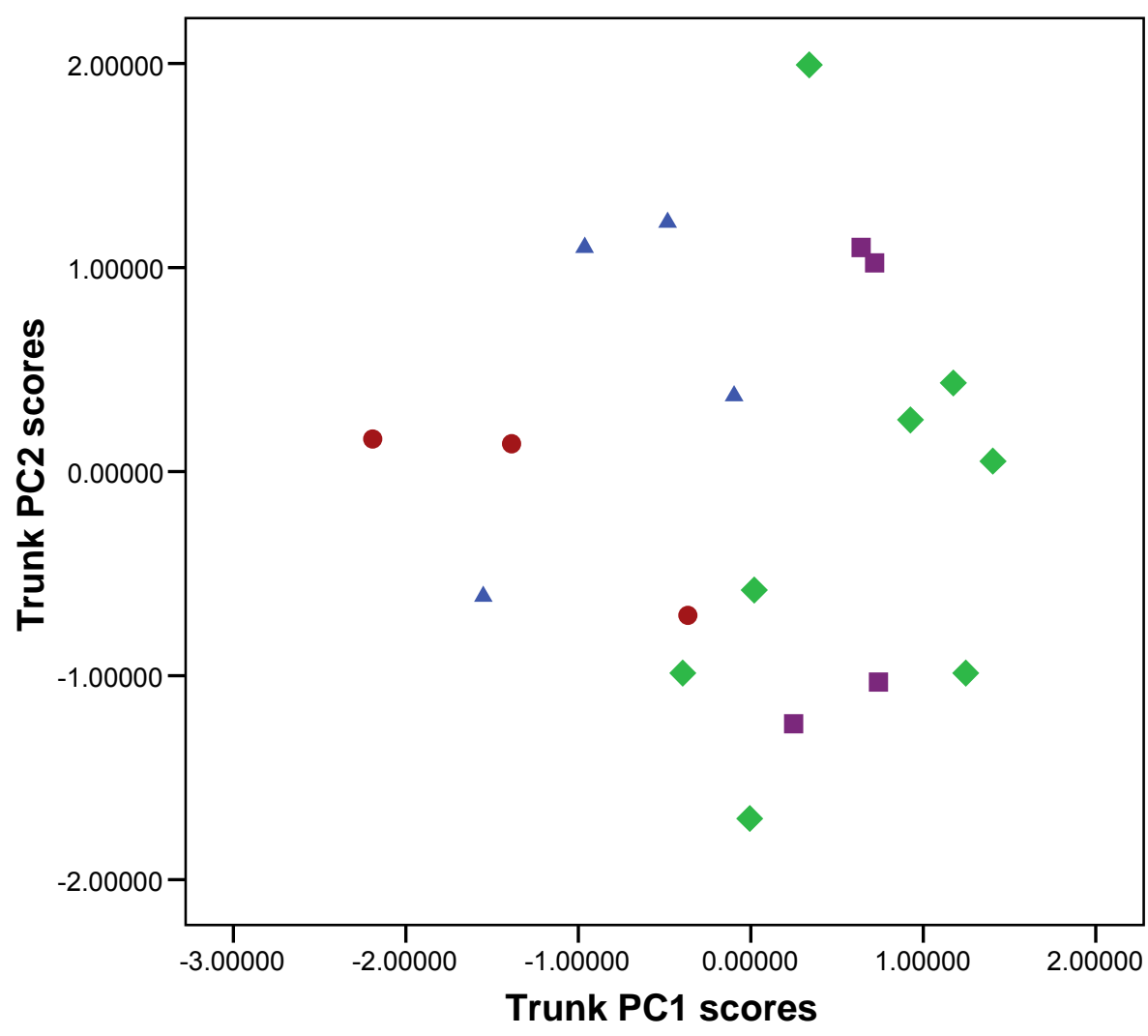

- F pre $2000 \mathrm{BP}, \mathrm{N}=4$ $F$ post $2000 \mathrm{BP}, \mathrm{N}=8$ $\mathrm{M}$ pre $2000 \mathrm{BP}, \mathrm{N}=3$

$M$ post 2000BP, $N=4$

\subsection{Scatterplot of PC1 and PC2 scores for the upper limb variables}

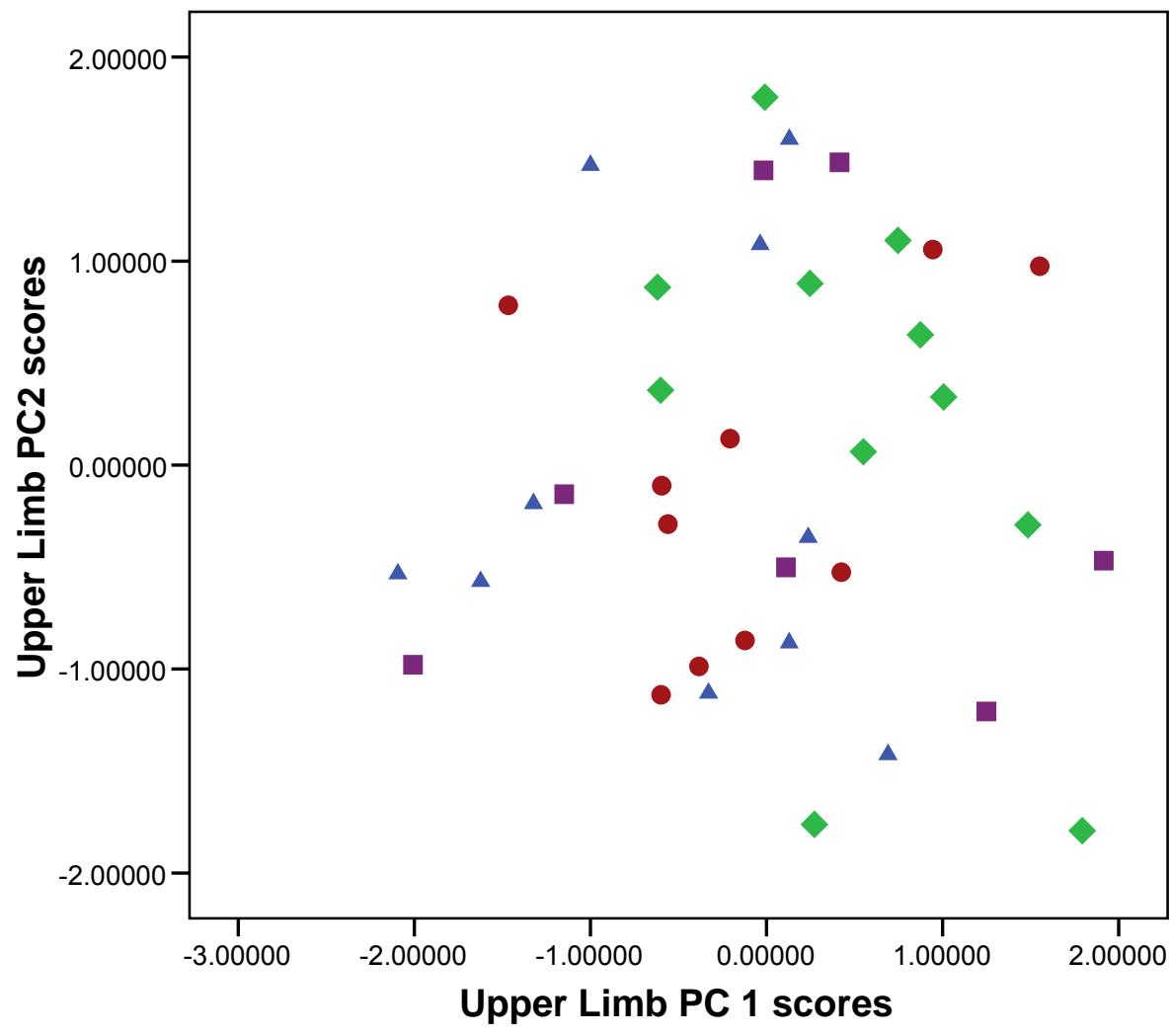

F pre $2000 \mathrm{BP}, \mathrm{N}=10$

$F$ post $2000 \mathrm{BP}, \mathrm{N}=11$ $M$ pre $2000 \mathrm{BP}, \mathrm{N}=10$

$M$ post $2000 \mathrm{BP}, \mathrm{N}=7$ 
Figure 4.28. Scatterplot of PC1 and 2 scores for the lower limb variables

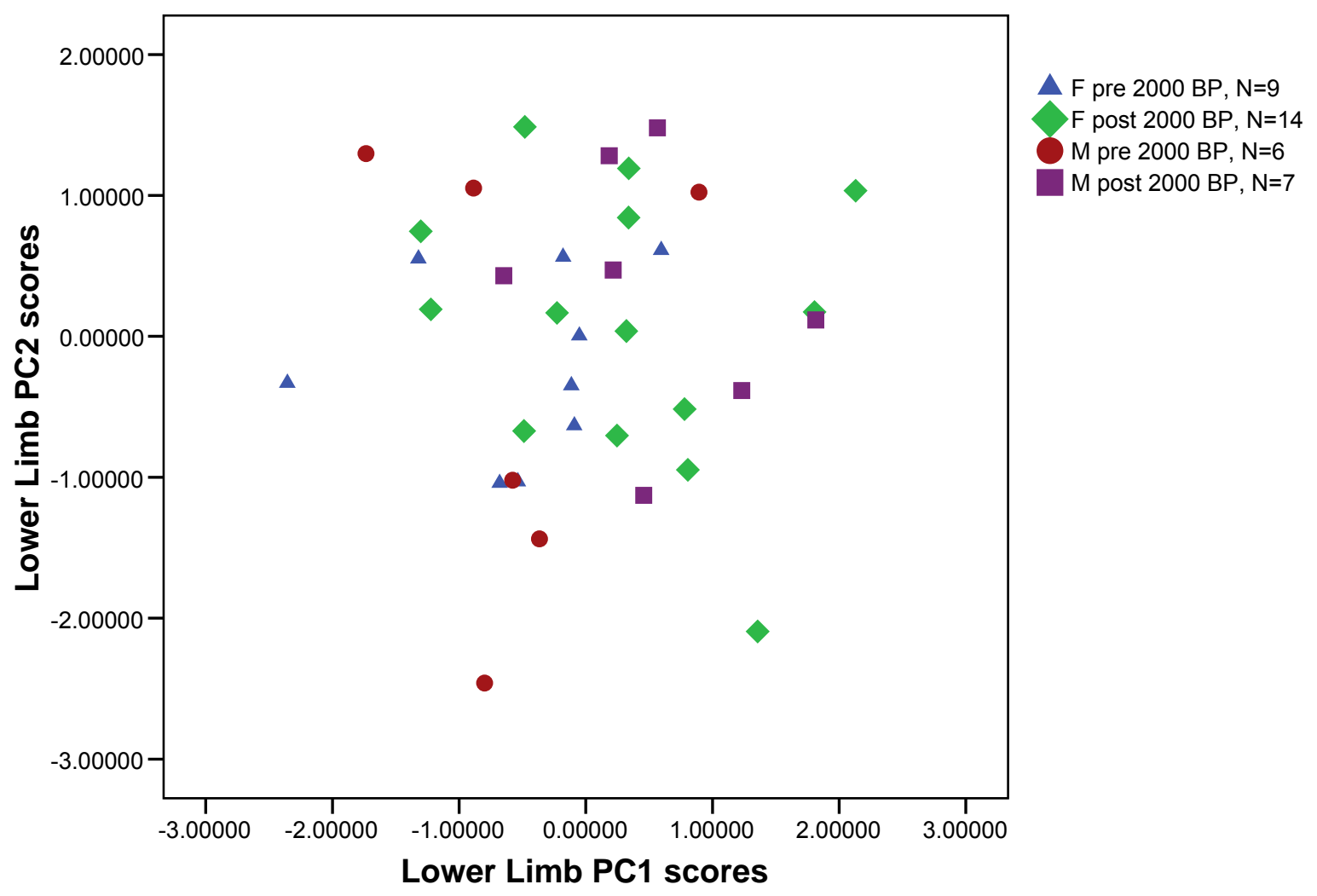

\subsubsection{Curve Estimation Regression}

Linear and quadratic curve regression analysis helped to elucidate temporal patterns in the osteometric data that were not evident when the sample was divided into subgroupings for the principal component analysis. The regression of the PC scores against ${ }^{14} \mathrm{C}$ date suggests that there is a significant relationship between size of the trunk in males $\left(\mathrm{r}^{2}=\right.$ $0.971, \mathrm{p}=0.007)$ and females $\left(\mathrm{r}^{2}=0.631, \mathrm{p}=0.011\right)$. Although the male relationship is stronger, trunk size undergoes a significant decrease around 3500 BP for both sexes (Figure 4.29). Just after $2000 \mathrm{BP}$ trunk size rebounds to pre-3500 BP levels and then increases significantly in the last 500 years. The fact that over half (total $n=11$, females $=7$, males $=4$ ) of the trunk sample dates to within the last 1000 years, may be influencing the shape of the 
quadratic curve and the apparent changes in trunk size. Although a reduction in $\mathrm{PC} 1$ values is evident, the concentration of positive post-1000 BP scores may be driving the curve and artificially enhancing the magnitude of the changes in truck size. A temporal pattern is not expressed for trunk shape. The relationship between $\mathrm{PC} 2$ and ${ }^{14} \mathrm{C}$ date is very weak for both females $\left(r^{2}=0.151, p=0.480\right)$ and males $\left(r^{2}=0.079, p=0.849\right)$. Trunk shape appears to be uniformly variable through time, with shape becoming more variable in only the last 500 years (Figure 4.30).

Figure 4.29. Scatterplot of regression scores for trunk variables: $\mathrm{PC} 1$ vs. ${ }^{14} \mathrm{C}$. Females $=$ dashed line, males $=$ solid line.

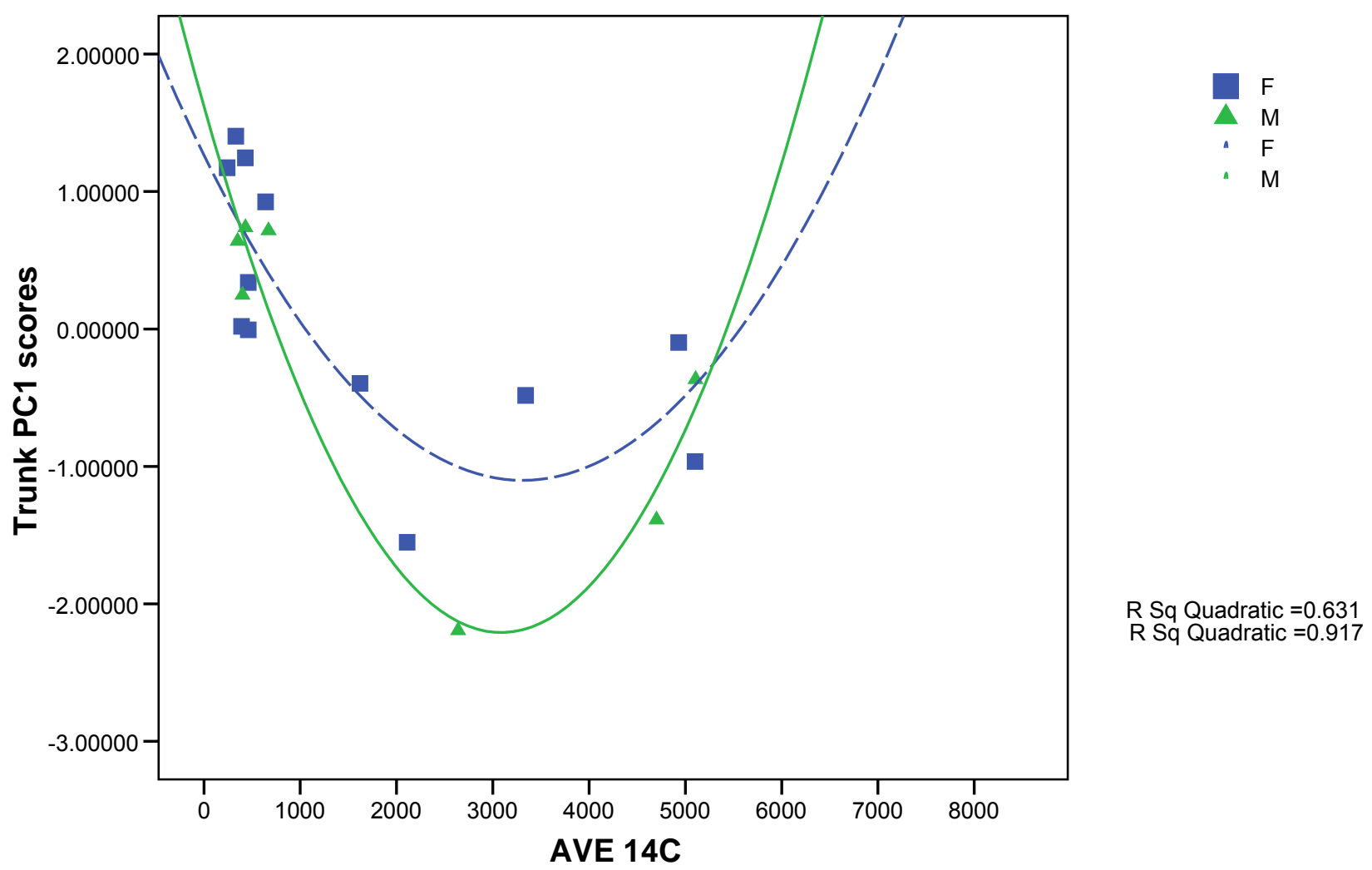


Figure 4.30. Scatterplot of regression scores for trunk variables: PC2 vs. ${ }^{14} \mathrm{C}$. Females $=$ dashed line, males $=$ solid line.

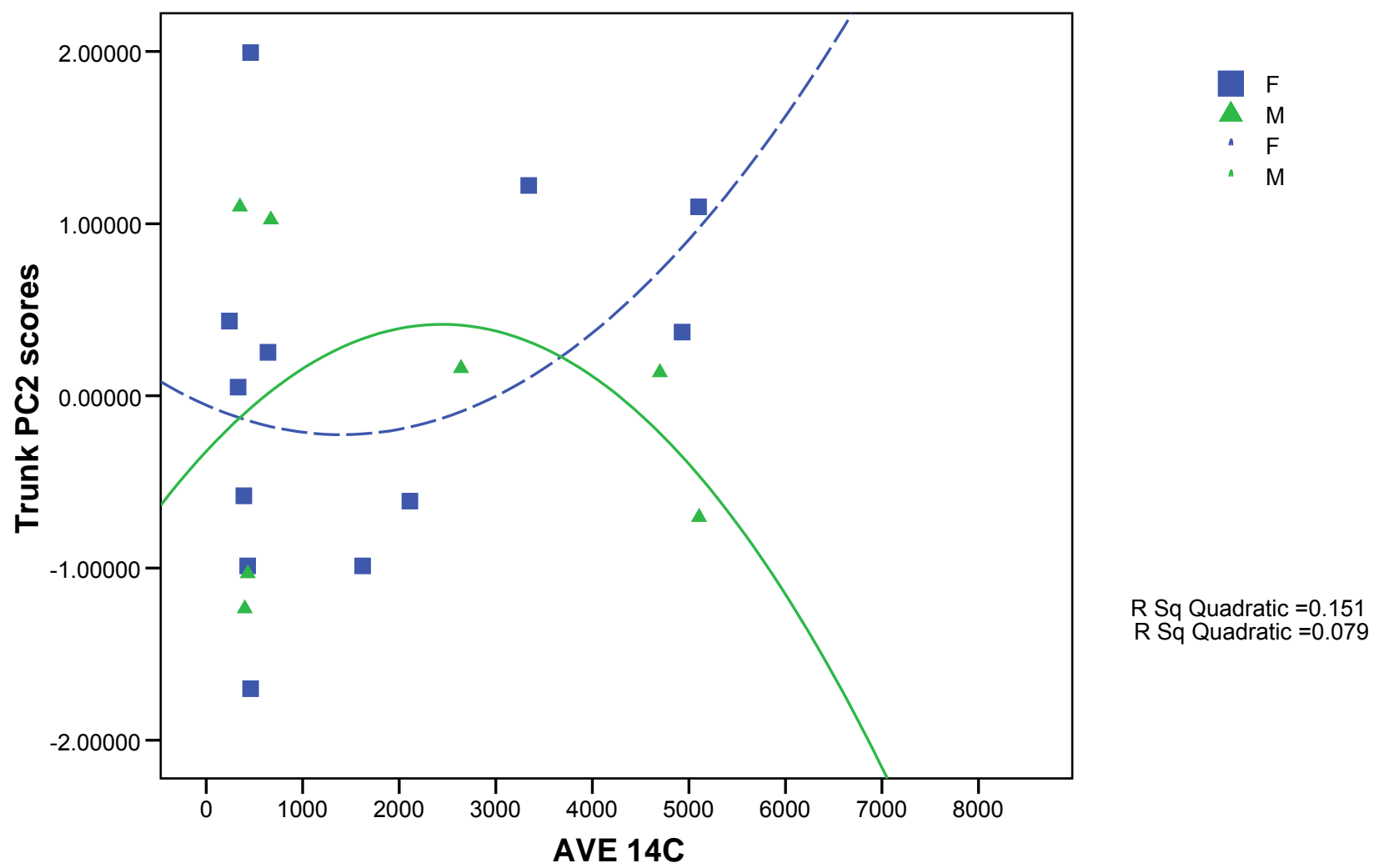

The upper limb regression analysis produced a significant relationship only for females, but males show a parallel shift in size through time. The upper limb size components follow the same decrease in size around $3500 \mathrm{BP}$ as was observed for the trunk, but the relationship between PC 1 and ${ }^{14} \mathrm{C}$ date is significant for females $\left(\mathrm{r}^{2}=0.337, \mathrm{p}=\right.$ $0.014)$ and not males $\left(r^{2}=0.203, p=0.204\right)$ (Figure 4.31). The period between 3500 to 2000 BP sees a general decrease in the size of the upper limb segments, yet a number of individuals in this period fall within the range observed prior to $3500 \mathrm{BP}$. After $2000 \mathrm{BP}$ upper limb size rebounds and continues to increase, with the most significant increases taking place in the past 500 years. With the exception of one male individual, after $2000 \mathrm{BP}$ no individuals possess upper limbs that fall below the pre-3500BP size range. Again, the 
regression analysis of the PC2 scores did not yield any significant results for females $\left(\mathrm{r}^{2}=\right.$ $0.027, p=0.784)$ or males $\left(r^{2}=0.103, p=0.469\right)$, indicating that upper limb shape remains stable through time (Figure 4.32).

Size changes in the lower limb through time are evident for both sexes. The regression of the $\mathrm{PC} 1$ scores for the lower limb against ${ }^{14} \mathrm{C}$ date yields significant results for both females $\left(\mathrm{r}^{2}=0.321, \mathrm{p}=0.021\right)$ and males $\left(\mathrm{r}^{2}=0.521, \mathrm{p}=0.025\right)$. Like the upper limb, the lower limb variable set displays a drop in body size between 3500 and $2000 \mathrm{BP}$, with size rebounding to and exceeding pre-2000 BP levels within the past 500 years (Figure 4.33). The lower limb shape components do not show change through time (females $r^{2}=0.029, p=$ 0.743 , and males $r^{2}=0.27, p=0.870$ ) (Figure 4.34).

Figure 4.31. Scatterplot of regression scores for upper limb variables: PC1 vs. ${ }^{14} \mathrm{C}$. Females $=$ dashed line, males $=$ solid line .

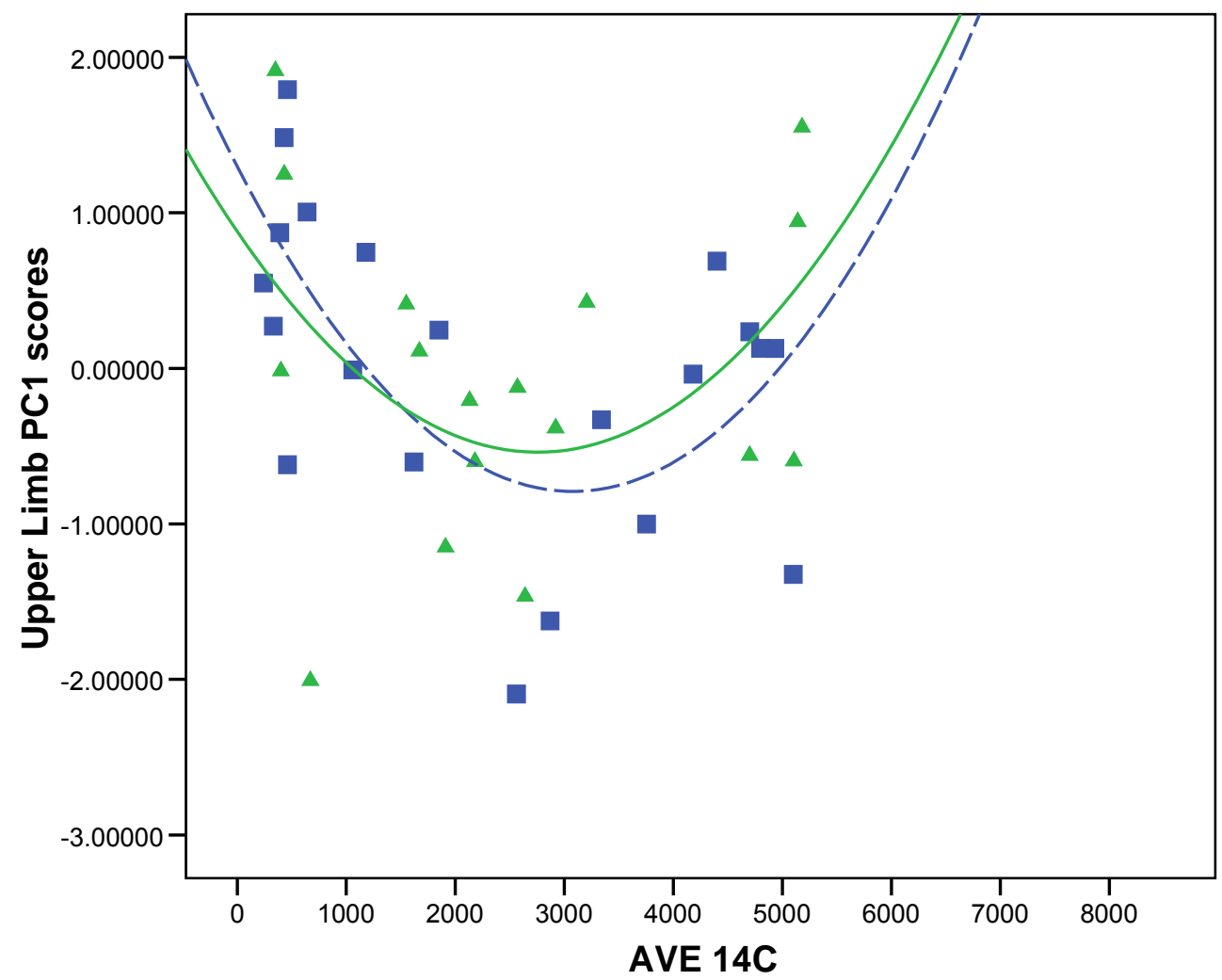

R Sq Quadratic $=0.377$ R Sq Quadratic $=0.203$ 
Figure 4.32. Scatterplot of regression scores for upper limb variables: PC2 vs. ${ }^{14} \mathrm{C}$. Females $=$ dashed line, males $=$ solid line .

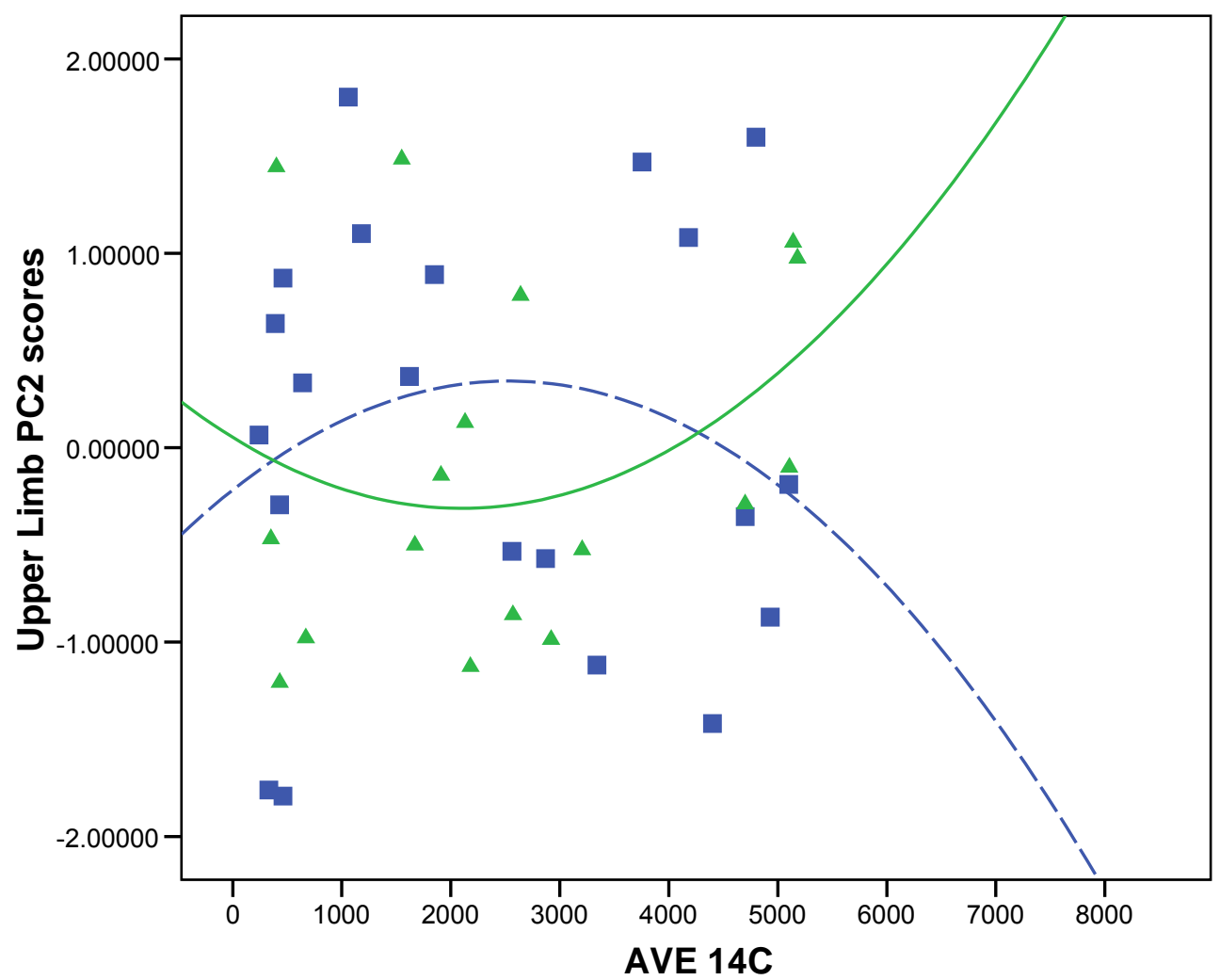

R Sq Quadratic $=0.027$

Figure 4.33. Scatterplot of regression scores for lower limb variables: PC1 vs. ${ }^{14} \mathrm{C}$. Females $=$ dashed line, males $=$ solid line .

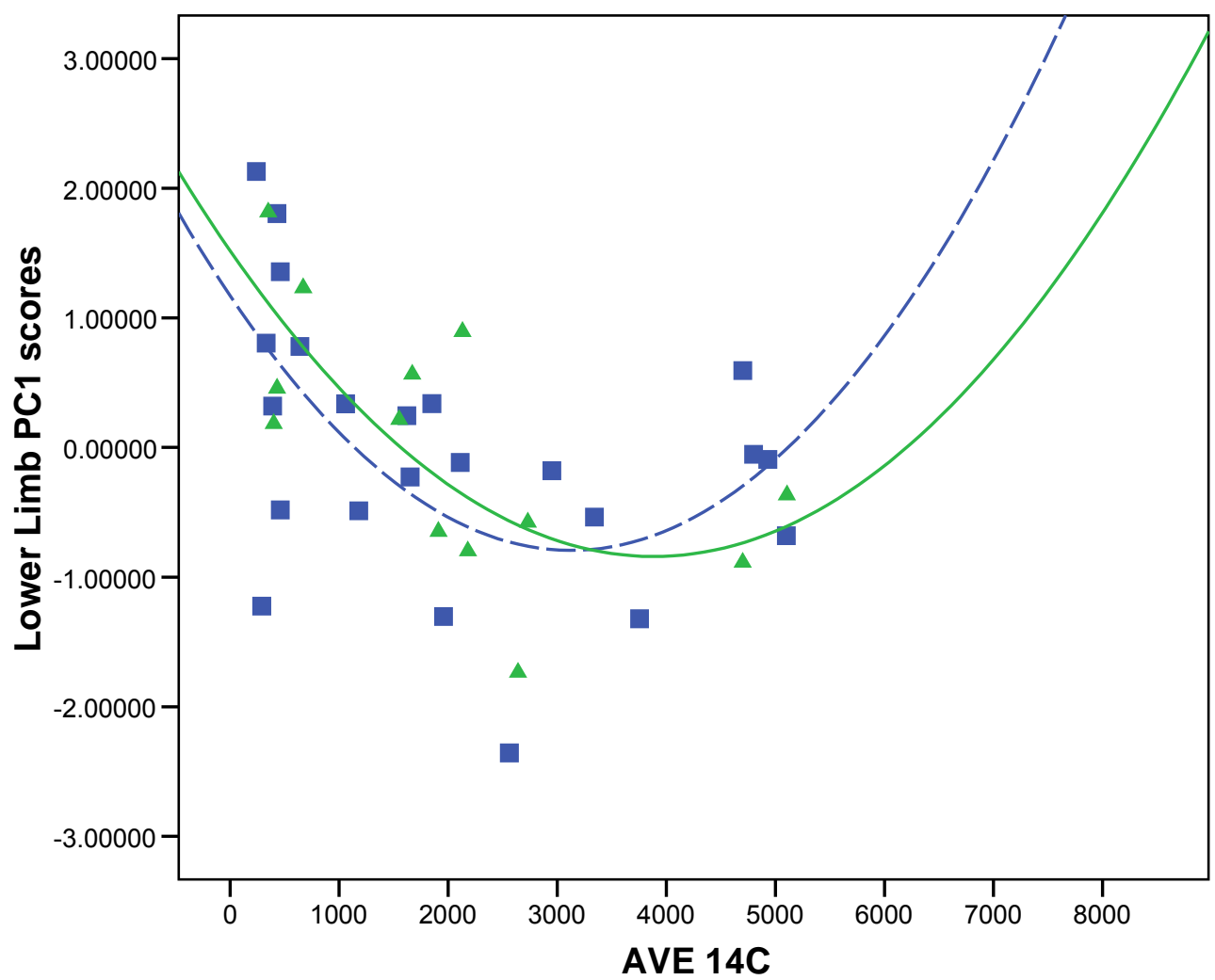

R Sq Quadratic $=0.321$ R Sq Quadratic $=0.521$ 
Figure 4.34. Scatterplot of regression scores for lower limb variables: PC2 vs. ${ }^{14} \mathrm{C}$. Females $=$ dashed line, males $=$ solid line .

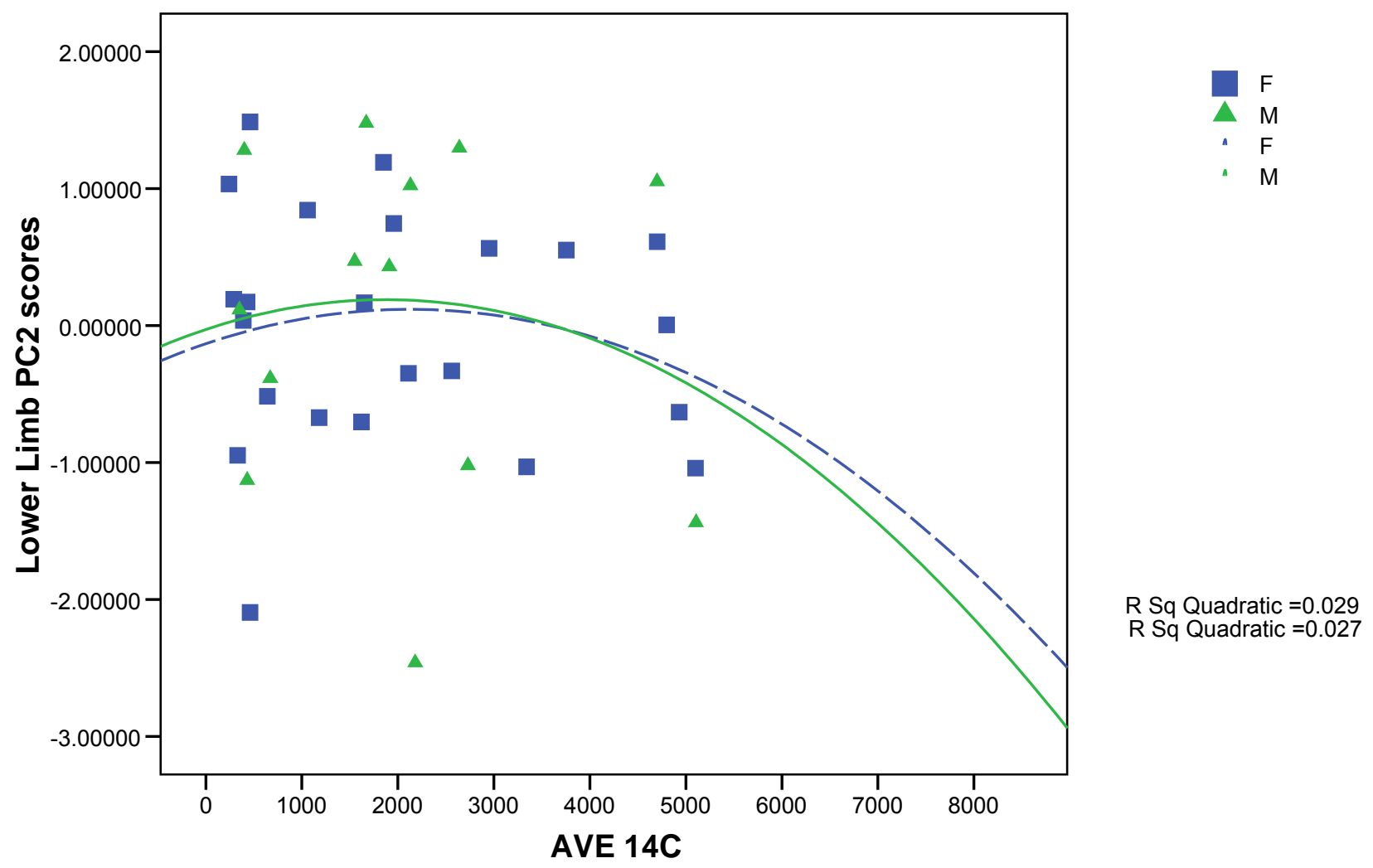

\subsubsection{Comparisons with Other South African Samples}

Osteometric data collected by Lundy (1986) on three South African Bantu-speaking skeletal samples (Cape Nguni, Natal Nguni, and Sotho) from the Raymond A. Dart Collection are compared with the Eastern Cape Later Stone Age postcranial metric sample. Summary statistics (means and standard deviations) were available for a selection of osteometric variables. T-tests were used to make comparisons between each of the four Eastern Cape sub-samples and available osteometric data.

The results of the comparative analysis support the broader osteometric findings of this research. The pre-2000 BP males and females differ significantly from the Cape Nguni, Natal Nguni, and Sotho samples for all osteometric variables (Table 4.11). A substantial 
portion of the variables show statistical differences between the post-2000 BP males and females and the comparative samples. Post-2000 BP females do not differ significantly from Cape Nguni females in tibial length (Table 4.11). Similarly, post-2000 BP males are similar to the Cape Nguni and Sotho samples for humeral head diameter, and the Sotho sample for tibial length. As noted in the postcranial multivariate results, recent individuals in the post2000 BP sub-group are of larger body size than the pre-2000 BP individuals and some of their older post-2000 BP counterparts. This observed increase in body size likely contributed to bringing the post-2000 BP group more in line with some of the South African Bantuspeaking samples for selected variables. 


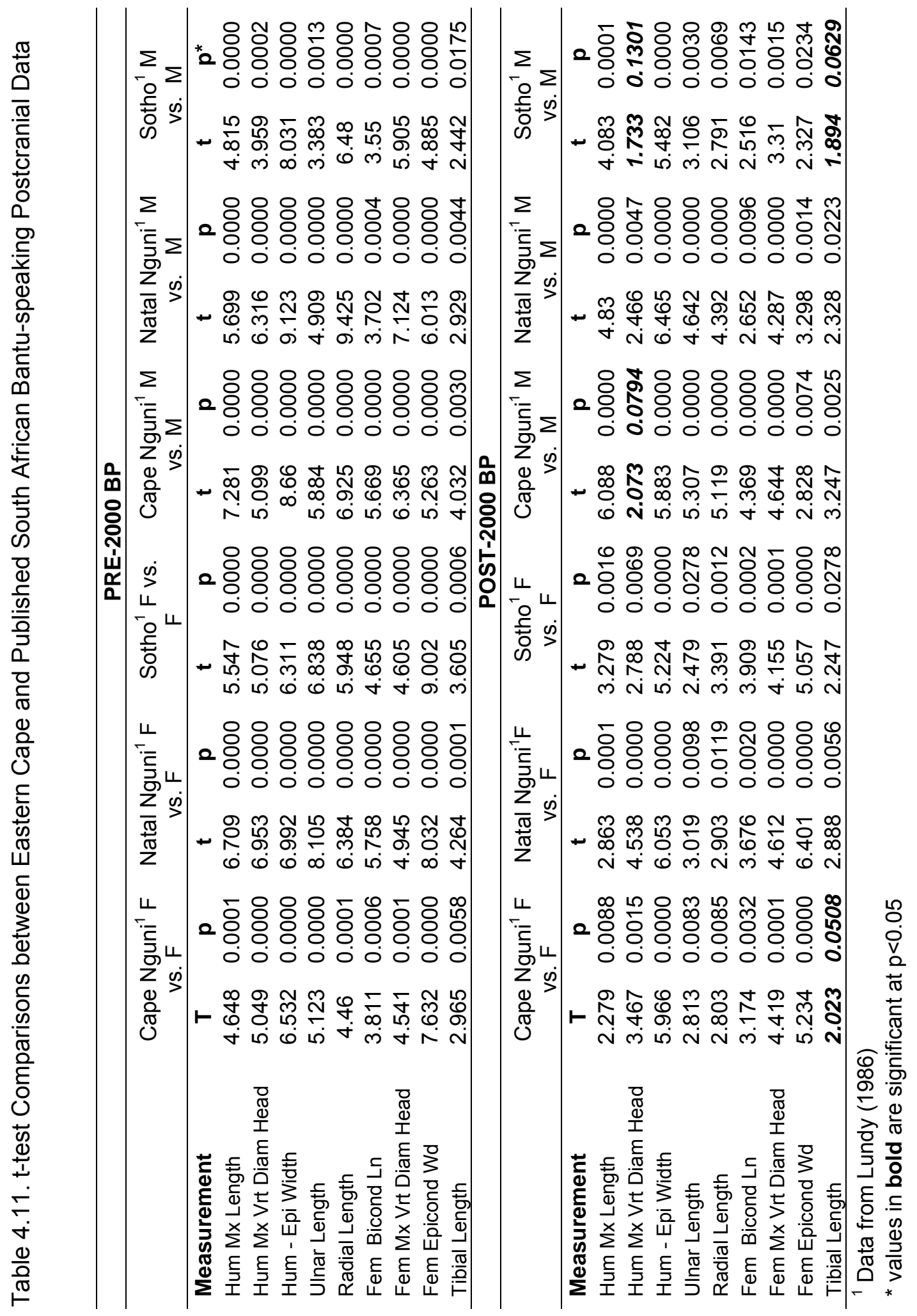




\subsection{Summary of Metric Results}

The absence of any clear patterns pertaining to cranial, dental and postcranial size and shape in the principal component results illustrates the difficulties of applying this statistical approach to a single, relatively small sample. Dividing the sample into subgroups, as is necessary for extraction of principal component scores, dilutes the results, making patterns in the data less evident. The regression of principal component scores against radiocarbon date allows the quantitative results to be viewed along a continuum rather than as a dichotomy. This approach helps to facilitate a more meaningful interpretation of the multivariate results. Even though this approach has its flaws, commonalities in the univariate results throughout the skeleton reinforce the validity of the multivariate findings.

A general pattern of slight to moderate size changes, without significant accompanying changes in skeletal form is present throughout the skeleton. The congruity in the timing of the observed reduction in size between 3500 and 2000 BP throughout the skeleton, with the return to pre-3500 BP levels suggests that environmental factors may be the cause. Although some shape changes are evident, these changes are weak and sexspecific. They are restricted to the vault and mandibular regions of the cranium, and do not correlate strongly with time signifying that genetic homogeneity was maintained in the Eastern Cape throughout the Holocene.

\subsection{Population Genetic Analysis}

The results of the Relethford-Blangero analysis point to changing patterns of population movement and group interaction across time in the Eastern Cape region. Prior to 2000 BP, it appears that Eastern Cape foragers were rather insular. The large negative residual value indicates that pre-2000 BP Eastern Cape foragers received significantly less 
than an expected average level of gene flow (Table 4.12). The large positive residual value suggests that the situation changed after $2000 \mathrm{BP}$, with the post-2000 BP group receiving significantly greater than average gene flow from extra-regional sources. These results indicate increased heterogeneity after $2000 \mathrm{BP}$, apparently due to the migration of nonlocals into the Eastern Cape region. However, it is also possible that the significant difference in levels of gene flow between the pre- and post-2000 BP groups may be a consequence of the movement of indigenous groups from outside the Eastern Cape, but still local to the greater southern South African Cape region. Even though the results of the Relethford-Blangero analysis suggest an increase in genetic variation after $2000 \mathrm{BP}$, the difference between the pre-2000 BP and post-2000 BP observed genetic variance values is not statistically significant.

Table 4.12. Relethford and Blangero analysis of gene flow or genetic drift

\begin{tabular}{lccccccc}
\hline Population & r(ii) & $\begin{array}{c}\text { Observed } \\
\text { variance }\end{array}$ & $\begin{array}{c}\text { Expected } \\
\text { variance }\end{array}$ & Residual & SE & $\begin{array}{c}\boldsymbol{t} \\
\text { (d.f. }=\text { 15) }\end{array}$ & $\boldsymbol{P}$ \\
\hline Pre-2000 BP & 0.03616 & 0.773 & 0.943 & -0.170 & 0.016766 & 10.12 & 0.0000 \\
Post-2000 BP & 0.01419 & 1.1 & 0.964 & 0.136 & 0.013374 & 10.17 & 0.0000 \\
\hline
\end{tabular}

Mean Within-group Phenotypic Variance $=0.955$

The impact of effective population size estimates on the estimates of gene flow must also be considered. While there is evidence that the distribution of South African populations changed after $2000 \mathrm{BP}$ (Binneman 1998; Jerardino et al. 2008) it is difficult to arrive at a confident estimate of effective population size for mobile groups like LSA foragers and herders. The influence of the population size on the estimates of gene flow was explored by running a series of Relethford-Blangero analyses with different population estimates for the pre- and post-2000 BP subgroups. As Figure 4.35 indicates, increasing the 
post-2000 BP effective population size estimate results in a reduction in the estimated levels of gene flow. This suggests that if population size increased significantly after $2000 \mathrm{BP}$ then increases in genetic variance may be the result of genetic drift, not gene flow.

Figure 4.35. The impact of effective population size estimates on estimates of gene flow.

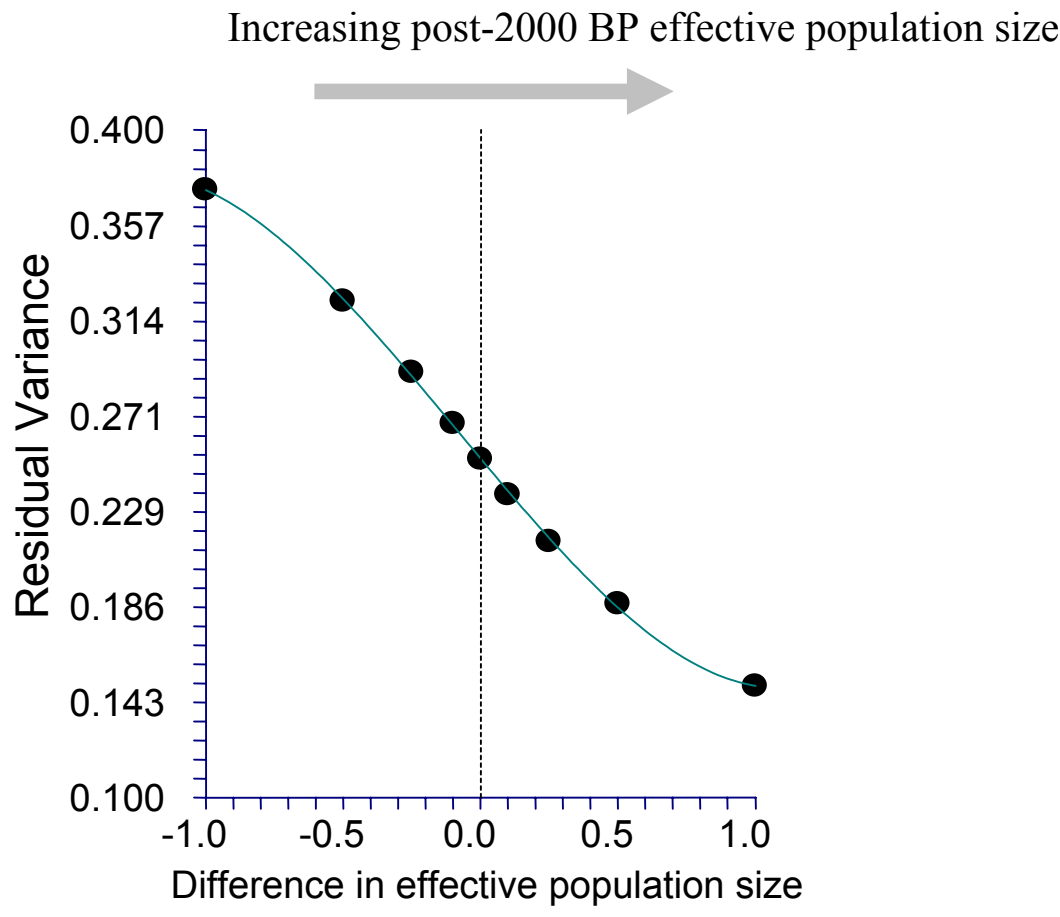

The results of the PC analysis of the variables used in the Relethford-Blangero analysis are consistent with those of the other skeletal PC analyses. Pre- and post-2000 BP scores overlap considerably (no significant differences) for all PCs except PC1. Since all of the PC1 variables are positive, this component can be interpreted as reflecting overall cranial size. As illustrated in Figure 4.35, some temporal patterning is evident along the PC1 axis with pre-2000 BP individuals clustering more towards the negative end and post-2000 BP individuals falling towards the positive end of the spectrum. This finding is echoed in the MANOVA results which indicate that there is a significant difference between the pre-2000 
BP and post-2000 BP samples for only PC1 (Table 4.13). Variance explained by the sixteen PCs is presented in Table 4.14. Thus, a significant change in size is noted through time among the Eastern Cape sample, with crania becoming larger after 2000BP. However, the absence of any changes in cranial shape supports the possibility that the significant increase in gene flow that is apparent after $2000 \mathrm{BP}$ is the result of movement of rather closely related South African Cape groups into the Eastern Cape.

Figure 4.36. Plot of PC1 and PC2 for the PCA of the Relethford-Blangero craniometric variables $(\bullet=$ pre-2000 BP individuals; $\Delta=$ post-2000 BP individuals).

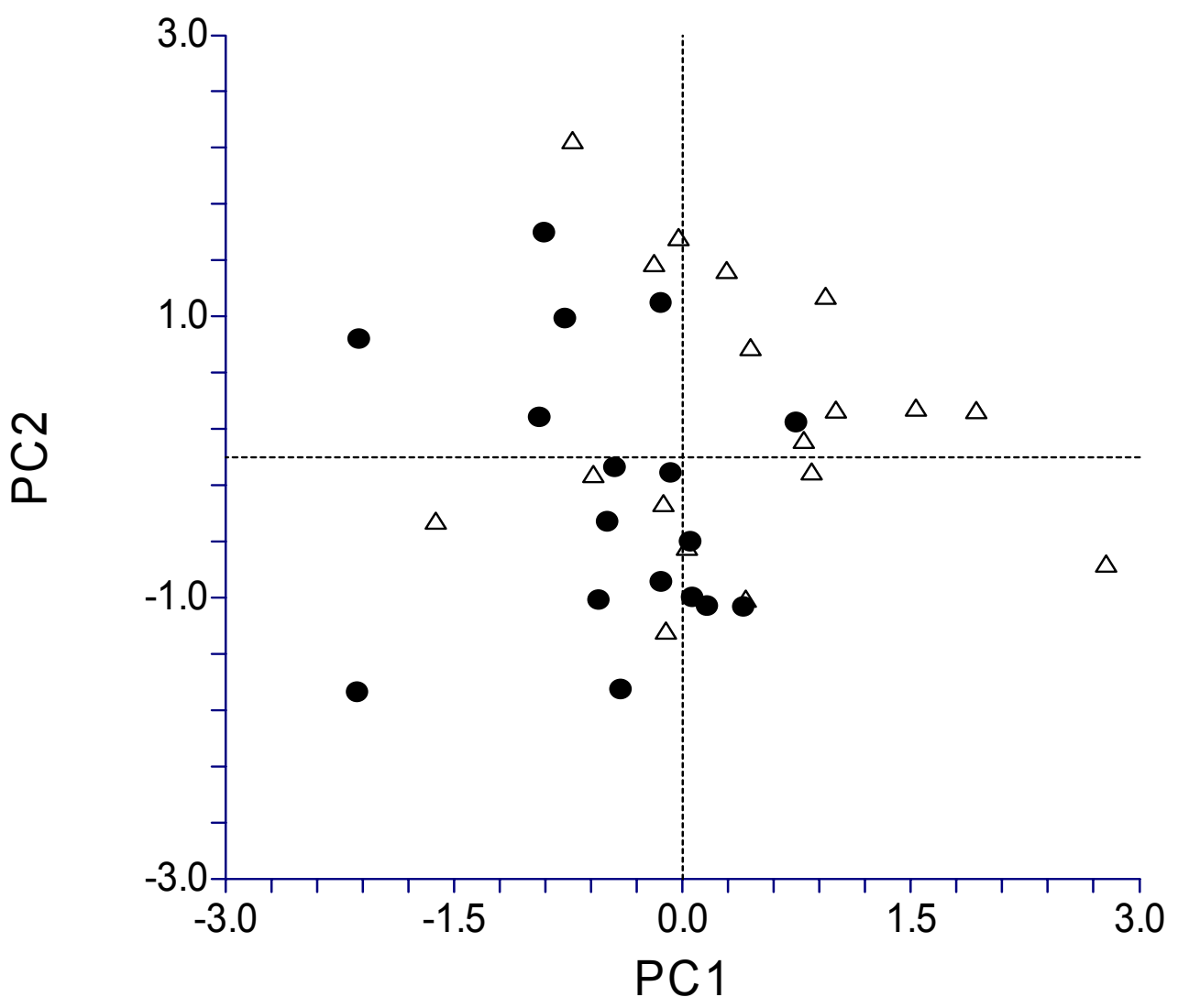


Table 4.13. Results of multivariate analysis of variance (MANOVA) of the first 8 principal components of the PCA conducted on the cranial variables used in the Relethford-Blangero analysis.

\begin{tabular}{llll}
\hline & Df & F & Significance $(p<0.05)$ \\
\hline Wilks' Lambda & 8 & 1.86 & 0.11 \\
PC1 & 1 & $\mathbf{7 . 9 6}$ & $\mathbf{0 . 0 0 8}$ \\
PC2 & 1 & 2.59 & 0.12 \\
PC3 & 1 & 0.78 & 0.39 \\
PC4 & 1 & 0.01 & 0.93 \\
PC5 & 1 & 0.15 & 0.70 \\
PC6 & 1 & 0.53 & 0.47 \\
PC7 & 1 & 0.09 & 0.76 \\
PC8 & 1 & 1.80 & 0.19
\end{tabular}

Table 4.14. Characteristics of the 16 principal components of the PCA on the cranial variables used in the Relethford-Blangero analysis.

\begin{tabular}{llll}
\hline & Eigenvalue & \% of Variance & Cumulative \% \\
\hline PC1 & 5.486 & 35.325 & 35.325 \\
PC2 & 2.261 & 14.558 & 49.883 \\
PC3 & 1.641 & 10.565 & 60.448 \\
PC4 & 1.415 & 9.114 & 69.562 \\
PC5 & 1.173 & 7.553 & 77.115 \\
PC6 & .987 & 6.358 & 83.473 \\
PC7 & .870 & 5.602 & 89.075 \\
PC8 & .539 & 3.468 & 92.543 \\
PC9 & .493 & 3.176 & 95.719 \\
PC10 & .363 & 2.336 & 98.055 \\
PC11 & .216 & 1.389 & 99.444 \\
PC12 & .078 & .501 & 99.945 \\
PC13 & .006 & .037 & 99.982 \\
PC14 & .002 & .014 & 99.996 \\
PC15 & .001 & .003 & 99.999 \\
PC16 & .000 & .001 & 100.000 \\
\hline
\end{tabular}




\subsection{Cranial Discrete Traits}

The dichotomous nature of the cranial discrete data does not lend itself well to the type of complex statistical manipulation that is used in the analysis of the skeletal metric data. As discussed in Chapter 3, only univariate analyses are conducted on the cranial discrete variables. Frequency data of the cranial discrete trait variables are provided in Table 4.15 .

\subsubsection{Univariate Analysis}

Possible differences in cranial discrete traits in the Eastern Cape sample are explored by comparing trait frequencies by sex and time period. Out of the forty-four bilateral and eight midline traits significant differences are observed between males and females for only two traits: partial pterygoalar bridge and apical ossification. Pearson $\chi^{2}$ values for these two traits suggest that the differences are significant, yet the accompanying Phi values indicate that the magnitude of differences is low (Table 4.16).

The analysis of cranial discrete trait frequencies across time also does not produce significant results. Only four traits generate significant chi-square values (Table 4.17). Pearson $\chi^{2}$ values and accompanying Phi values suggest that while the change in frequency of tympanic dehiscence, trace pterygoalar bridge, and all manifestations of pterygoalar bridging through time is significant, this correlation is not strong. Os japonicum is the only trait that supports a significant change through time. Only one out of the thirty-four pre-2000 BP individuals displays this trait, while it is present in twelve out of the forty-five post-2000 $\mathrm{BP}$ individuals. When the locations of individuals possessing this trait are plotted by longitude and latitude co-ordinates a pattern is evident (Figure 4.36). All but three of the individuals displaying the os japonicum trait are buried along the coast in the Cape St. 
Table 4.15. Cranial Discrete Frequency Data. Trait definitions are provided in Appendix 6.

\begin{tabular}{|c|c|c|c|c|c|c|c|c|}
\hline \multirow{3}{*}{ Trait } & \multicolumn{4}{|c|}{ Pre-2000 } & \multicolumn{4}{|c|}{ Post-2000 } \\
\hline & \multicolumn{2}{|c|}{ Females } & \multicolumn{2}{|c|}{ Males } & \multicolumn{2}{|c|}{ Females } & \multicolumn{2}{|c|}{ Males } \\
\hline & $\mathrm{P} / \mathrm{N}$ & $\%$ & $\mathrm{P} / \mathrm{N}$ & $\%$ & $\mathrm{P} / \mathrm{N}$ & $\%$ & $\mathrm{P} / \mathrm{N}$ & $\%$ \\
\hline Partial Metopic Suture & $1 / 11$ & 9.1 & $1 / 13$ & 7.7 & $3 / 16$ & 18.8 & $4 / 11$ & 36.4 \\
\hline Full Metopic Suture & $0 / 12$ & 0 & $1 / 13$ & 7.7 & $0 / 16$ & 0 & $1 / 11$ & 9.1 \\
\hline Supraorbital Notch & $13 / 22$ & 59.1 & $12 / 23$ & 52.2 & $21 / 30$ & 70 & $17 / 22$ & 77.3 \\
\hline Supraorbital Foramen & $9 / 22$ & 40.9 & $10 / 23$ & 43.5 & $11 / 30$ & 36.7 & $5 / 22$ & 22.7 \\
\hline Supratrochlear Notch & $3 / 21$ & 14.3 & $3 / 24$ & 12.5 & $10 / 30$ & 33.3 & $5 / 22$ & 22.7 \\
\hline Infraorbital Suture & $8 / 14$ & 57.1 & $9 / 15$ & 60 & $17 / 28$ & 60.7 & $4 / 13$ & 30.8 \\
\hline Infraorbital Foramen & $0 / 19$ & 0 & $0 / 19$ & 0 & $1 / 29$ & 3.4 & $0 / 22$ & 0 \\
\hline Parietal Foramen & $12 / 20$ & 60 & $11 / 23$ & 47.8 & $21 / 31$ & 67.7 & $16 / 22$ & 72.7 \\
\hline Epiteric Bone & $3 / 19$ & 15.8 & $0 / 21$ & 0 & $4 / 30$ & 13.3 & $2 / 22$ & 9.1 \\
\hline Coronal Ossicle & $1 / 21$ & 4.8 & $2 / 21$ & 9.1 & $0 / 32$ & 0 & $2 / 21$ & 9.5 \\
\hline Bregmatic Bone & $1 / 11$ & 9.1 & $0 / 13$ & 0 & $0 / 16$ & 0 & $0 / 11$ & 0 \\
\hline Sagittal Ossicle & $0 / 10$ & 0 & $0 / 14$ & 0 & $0 / 16$ & 0 & $0 / 11$ & 0 \\
\hline Apical Bone & $1 / 9$ & 11.1 & $0 / 14$ & 0 & $3 / 16$ & 18.8 & $0 / 11$ & 0 \\
\hline Inca Bone & $0 / 10$ & 0 & $0 / 14$ & 0 & $0 / 16$ & 0 & $0 / 11$ & 0 \\
\hline Lambdoid Ossicle & $4 / 20$ & 20 & $14 / 28$ & 50 & $13 / 32$ & 40.6 & $8 / 22$ & 36.4 \\
\hline Asterionic Bone & $1 / 19$ & 5.3 & $4 / 25$ & 16 & $1 / 32$ & 3.1 & $0 / 21$ & 0 \\
\hline Ossicle Occipitomastoid Suture & $2 / 20$ & 10 & $4 / 25$ & 16 & $1 / 29$ & 3.4 & $1 / 22$ & 4.5 \\
\hline Parietal Notch Bone & $3 / 19$ & 15.8 & $5 / 21$ & 23.8 & $3 / 31$ & 9.7 & $3 / 21$ & 14.3 \\
\hline Condylar Canal Present & $5 / 14$ & 35.7 & $9 / 19$ & 47.4 & $15 / 24$ & 62.5 & $13 / 20$ & 65 \\
\hline Divided Hypoglossal Canal & $2 / 20$ & 10 & $2 / 20$ & 10 & $2 / 29$ & 6.9 & $2 / 22$ & 9.1 \\
\hline Tympanic Dehiscence & $12 / 20$ & 60 & $13 / 21$ & 61.9 & $12 / 31$ & 38.7 & $3 / 22$ & 13.6 \\
\hline Foramen Spinosum Incomplete & $7 / 16$ & 43.8 & $8 / 19$ & 42.1 & $9 / 28$ & 32.1 & $9 / 22$ & 40.9 \\
\hline Foramen Ovale Incomplete & $0 / 18$ & 0 & $0 / 22$ & 0 & $1 / 28$ & 3.6 & $1 / 21$ & 4.8 \\
\hline Pterygospinous Bridge - Trace & $1 / 16$ & 6.3 & $4 / 21$ & 19 & $6 / 28$ & 21.4 & $3 / 20$ & 15 \\
\hline Pterygospinous Bridge - Partial & $0 / 16$ & 0 & $1 / 21$ & 4.8 & $2 / 28$ & 7.1 & $0 / 20$ & 0 \\
\hline Pterygospinous Bridge - Complete & $0 / 16$ & 0 & $1 / 21$ & 4.8 & $0 / 28$ & 0 & $0 / 20$ & 0 \\
\hline Pterygospinous Bridge - All & $1 / 16$ & 6.3 & $6 / 21$ & 28.6 & $8 / 28$ & 28.6 & $3 / 20$ & 15 \\
\hline Pterygoalar Bridge - Trace & $3 / 18$ & 16.7 & $4 / 22$ & 18.2 & $11 / 29$ & 37.9 & $8 / 22$ & 36.4 \\
\hline Pterygoalar Bridge - Partial & $1 / 18$ & 5.6 & $2 / 22$ & 9.1 & $1 / 29$ & 3.4 & $7 / 22$ & 31.8 \\
\hline Pterygoalar Bridge - Complete & $0 / 18$ & 0 & $1 / 22$ & 4.5 & $0 / 29$ & 0 & $0 / 22$ & 0 \\
\hline Pterygoalar Bridge - All & $4 / 18$ & 22.2 & $7 / 22$ & 31.8 & $11 / 29$ & 37.9 & $15 / 22$ & 68.2 \\
\hline Palatine Torus & $0 / 12$ & 0 & $1 / 10$ & 10 & $1 / 16$ & 6.3 & $2 / 11$ & 18.2 \\
\hline Auditory Exostoses & $0 / 21$ & 0 & $0 / 20$ & 0 & $0 / 31$ & 0 & $0 / 22$ & 0 \\
\hline Mastoid Foramen - Temporal & $11 / 18$ & 61.1 & $15 / 22$ & 68.2 & $18 / 31$ & 58.1 & $16 / 22$ & 72.7 \\
\hline Mastoid Foramen - Sutural & $7 / 18$ & 38.9 & $8 / 22$ & 36.4 & $6 / 31$ & 19.4 & $10 / 22$ & 45.5 \\
\hline Mastoid Foramen - Occipital & $6 / 18$ & 33.3 & $6 / 22$ & 27.3 & $12 / 31$ & 38.7 & $7 / 22$ & 31.8 \\
\hline Zygomaticofacial Foramen & $17 / 22$ & 77.3 & $18 / 21$ & 85.7 & $26 / 30$ & 86.6 & $15 / 21$ & 71.4 \\
\hline Mental Foramen Absent & $0 / 13$ & 0 & $2 / 28$ & 7.14 & $0 / 30$ & 0 & $0 / 17$ & 0 \\
\hline Accessory Mental Foramen & $4 / 26$ & 15.4 & $3 / 28$ & 10.7 & $0 / 30$ & 0 & $5 / 17$ & 29.4 \\
\hline Mylohyoid Bridge & $16 / 26$ & 61.5 & $14 / 24$ & 58.3 & $14 / 28$ & 50 & $12 / 17$ & 70.6 \\
\hline Mandibular Torus & $0 / 28$ & 0 & $0 / 28$ & 0 & $0 / 30$ & 0 & $0 / 18$ & 0 \\
\hline Frontal Lines & $14 / 24$ & 58.3 & $15 / 25$ & 60 & $19 / 32$ & 59.4 & $11 / 22$ & 50 \\
\hline Frontal Foramen & $7 / 21$ & 33.3 & $8 / 22$ & 36.4 & $18 / 30$ & 60 & $10 / 22$ & 45.5 \\
\hline Trochlear Spur & $5 / 21$ & 23.8 & $3 / 20$ & 15 & $0 / 30$ & 0 & $3 / 22$ & 13.6 \\
\hline Os Japonicum & $0 / 16$ & 0 & $1 / 18$ & 5.6 & $4 / 23$ & 17.4 & $8 / 22$ & 36.4 \\
\hline
\end{tabular}


Table 4.15 Continued

\begin{tabular}{lcc|cc|cc|cc}
\hline \multirow{2}{*}{\multicolumn{1}{c}{ Trait }} & \multicolumn{4}{c}{ Pre-2000 } & \multicolumn{3}{c}{ Post-2000 } \\
\cline { 2 - 10 } & \multicolumn{2}{c}{ Females } & \multicolumn{2}{c|}{ Males } & \multicolumn{2}{c}{ Females } & \multicolumn{2}{c}{ Males } \\
& $\mathrm{P} / \mathrm{N}$ & $\mathbf{\%}$ & $\mathrm{P} / \mathrm{N}$ & $\mathbf{\%}$ & $\mathrm{P} / \mathrm{N}$ & $\mathbf{\%}$ & $\mathrm{P} / \mathrm{N}$ & $\mathbf{\%}$ \\
\hline Parietal Process of the Temporal & $1 / 21$ & $\mathbf{4 . 8}$ & $0 / 23$ & $\mathbf{0}$ & $3 / 30$ & $\mathbf{1 0}$ & $1 / 22$ & $\mathbf{4 . 5}$ \\
Marginal Foramen & $0 / 21$ & $\mathbf{0}$ & $2 / 22$ & $\mathbf{9 . 1}$ & $0 / 29$ & $\mathbf{0}$ & $1 / 21$ & $\mathbf{4 . 8}$ \\
Spinobasal Bridge - Trace & $3 / 17$ & $\mathbf{1 7 . 6}$ & $5 / 23$ & $\mathbf{2 1 . 7}$ & $7 / 28$ & $\mathbf{2 5}$ & $6 / 21$ & $\mathbf{2 8 . 6}$ \\
Spinobasal Bridge - Partial & $1 / 17$ & $\mathbf{5 . 9}$ & $5 / 23$ & $\mathbf{2 1 . 7}$ & $6 / 28$ & $\mathbf{2 1 . 4}$ & $3 / 21$ & $\mathbf{1 4 . 3}$ \\
Spinobasal Bridge - Complete & $1 / 17$ & $\mathbf{5 . 9}$ & $1 / 23$ & $\mathbf{4 . 3}$ & $1 / 28$ & $\mathbf{3 . 6}$ & $3 / 21$ & $\mathbf{1 4 . 3}$ \\
Spinobasal Bridge - All & $5 / 17$ & $\mathbf{2 9 . 4}$ & $11 / 23$ & $\mathbf{4 7 . 8}$ & $14 / 28$ & $\mathbf{5 0}$ & $11 / 21$ & $\mathbf{5 2 . 4}$ \\
Apical ossification & $1 / 9$ & $\mathbf{1 1 . 1}$ & $1 / 10$ & $\mathbf{1 0}$ & $0 / 15$ & $\mathbf{0}$ & $5 / 11$ & $\mathbf{4 5 . 5}$ \\
\hline
\end{tabular}

Table 4.16. Results of Pearson chi-square analysis of Eastern Cape male and female cranial discrete trait frequencies

\begin{tabular}{|c|c|c|c|c|c|}
\hline Variable & Female & Male & $x^{2}$ & $\mathbf{p}$ & Phi \\
\hline \multicolumn{6}{|l|}{ BILATERAL } \\
\hline SONOTCH & $35 / 52$ & $29 / 45$ & 0.009 & 0.923 & \\
\hline SOFORAM & $20 / 52$ & $15 / 45$ & 0.275 & 0.6 & \\
\hline STNOTCH & $13 / 51$ & $8 / 46$ & 0.935 & 0.334 & \\
\hline IOSUT & $23 / 42$ & $13 / 28$ & 0.467 & 0.494 & \\
\hline IOFORAM & $1 / 48$ & $0 / 41$ & $0.000^{*}$ & $1.000^{*}$ & \\
\hline PFORAM & $33 / 51$ & $27 / 44$ & 0.113 & 0.736 & \\
\hline EPITER & $7 / 49$ & $2 / 43$ & $1.441^{*}$ & $0.230^{*}$ & \\
\hline CORONL & $1 / 53$ & $4 / 42$ & $1.423^{*}$ & $0.233^{*}$ & \\
\hline LAMBOSS & $17 / 52$ & $22 / 50$ & 1.38 & 0.24 & \\
\hline ASTRINB & $2 / 51$ & $4 / 46$ & $0.305^{*}$ & $0.581^{*}$ & \\
\hline OMSUTS & $3 / 49$ & $5 / 47$ & $0.186^{*}$ & $0.667^{*}$ & \\
\hline PARTNOTB & $6 / 50$ & $8 / 42$ & 0.879 & 0.349 & \\
\hline CONDCANABS & $20 / 38$ & $22 / 39$ & 0.111 & 0.739 & \\
\hline DIHYPOC & $4 / 49$ & $4 / 42$ & $0.000^{*}$ & $1.000^{*}$ & \\
\hline TYMPDIHS & $19 / 51$ & $16 / 43$ & 0 & 0.996 & \\
\hline FRSPINI & $16 / 44$ & $17 / 41$ & 0.232 & 0.63 & \\
\hline FOROVLI & $1 / 46$ & $1 / 43$ & $0.000^{*}$ & $1.000^{*}$ & \\
\hline PTSPBRTR & $7 / 44$ & $7 / 41$ & 0.021 & 0.885 & \\
\hline PTSPBRPRT & $2 / 44$ & 1.41 & $0.000^{*}$ & $1.000^{*}$ & \\
\hline PTSPBRCOM & $0 / 44$ & $1 / 41$ & $0.001^{*}$ & $0.972^{*}$ & \\
\hline PTSPBRALL & $9 / 44$ & $9 / 41$ & 0.028 & 0.866 & \\
\hline PTABRGTR & $14 / 47$ & $12 / 42$ & 0.07 & 0.791 & \\
\hline PTABRGPRT & $2 / 47$ & $9 / 49$ & 5.612 & 0.018 & 0.248 \\
\hline PTABRGCOM & $0 / 47$ & $1 / 44$ & $0.001^{*}$ & $0.974^{*}$ & \\
\hline PTABRGALL & $15 / 47$ & $22 / 44$ & 3.081 & 0.079 & \\
\hline AUDEXOS & $0 / 25$ & $0 / 42$ & $\mathrm{x}$ & $\mathrm{x}$ & \\
\hline MASTFRTEM & $29 / 49$ & $31 / 44$ & 1.286 & 0.257 & \\
\hline MASTFRSUT & $13 / 49$ & $18 / 44$ & 2.157 & 0.142 & \\
\hline MASTFROCC & $18 / 49$ & $13 / 44$ & 0.539 & 0.463 & \\
\hline ZYFFOR & $43 / 52$ & $33 / 42$ & 0.255 & 0.614 & \\
\hline
\end{tabular}


Table 4.16. Continued.

\begin{tabular}{|c|c|c|c|c|c|}
\hline Variable & Female & Male & $x^{2}$ & $\mathbf{p}$ & Phi \\
\hline \multicolumn{6}{|l|}{ BILATERAL } \\
\hline MENTFORABS & $0 / 56$ & $2 / 45$ & $0.766^{*}$ & $0.382^{*}$ & \\
\hline ACCMENTFOR & $4 / 56$ & $8 / 45$ & 2.695 & 0.101 & \\
\hline MYLHBRD & $30 / 54$ & $22 / 41$ & 0.034 & 0.854 & \\
\hline MANDTOR & $0 / 58$ & $0 / 46$ & $\mathrm{x}$ & $\mathrm{x}$ & \\
\hline FRONTLN & $33 / 56$ & $26 / 47$ & 0.136 & 0.712 & \\
\hline FRONFOR & $25 / 51$ & $18 / 44$ & 0.627 & 0.428 & \\
\hline TROCSPR & $5 / 51$ & $6 / 42$ & $0.118^{*}$ & $0.731^{*}$ & \\
\hline OSJAP & $4 / 39$ & $9 / 40$ & 2.153 & 0.142 & \\
\hline PARPROC & $4 / 41$ & $1 / 45$ & $0.603^{*}$ & $0.437^{*}$ & \\
\hline MARGFOR & $0 / 50$ & $3 / 43$ & $1.716^{*}$ & $0.190^{*}$ & \\
\hline SPBASBRTR & $10 / 45$ & $11 / 44$ & 0.095 & 0.758 & \\
\hline SPBASBRPRT & $7 / 45$ & $8 / 44$ & 0.109 & 0.741 & \\
\hline SPBASBRCOM & $2 / 45$ & $4 / 44$ & $0.204^{*}$ & $0.652^{*}$ & \\
\hline SPBASBRALL & $19 / 45$ & $22 / 44$ & 0.542 & 0.462 & \\
\hline \multicolumn{6}{|l|}{ MIDLINE } \\
\hline METOPOPART & $4 / 27$ & $5 / 24$ & $0.038^{*}$ & $0.846^{*}$ & \\
\hline METOPFULL & $0 / 28$ & $2 / 24$ & $0.696^{*}$ & $0.404^{*}$ & \\
\hline BRAGMATB & $1 / 27$ & $0 / 24$ & $0.000^{*}$ & $1.000^{*}$ & \\
\hline SAGITOSSIC & $0 / 26$ & $0 / 25$ & $x$ & $x$ & \\
\hline APICALBN & $4 / 25$ & $0 / 25$ & $2.446^{*}$ & $0.118^{*}$ & \\
\hline INCABONE & $0 / 26$ & $0 / 25$ & $\mathrm{x}$ & $\mathrm{x}$ & \\
\hline PALTORUS & $1 / 28$ & $3 / 21$ & $0.686^{*}$ & $0.407^{*}$ & \\
\hline APICOSS & $1 / 24$ & $6 / 21$ & $3.390^{*}$ & $0.066^{*}$ & \\
\hline
\end{tabular}

${ }^{*}$ expected cell count in $2 \times 2$ table $<5$ so used Continuity Correction values

Table 4.17. Results of Pearson chi-square analysis of Pre- and Post-2000 BP cranial discrete trait frequencies

\begin{tabular}{lccccc}
\hline Variable & Pre-2000 BP & Post-2000 BP & $\mathbf{x}^{\mathbf{2}}$ & $\mathbf{p}$ & Phi \\
\hline BILATERAL & & & & & \\
SONOTCH & $25 / 45$ & $38 / 52$ & 3.253 & 0.071 & \\
SOFORAM & $19 / 45$ & $16 / 52$ & 1.372 & 0.241 & \\
STNOTCH & $6 / 45$ & $15 / 52$ & 3.422 & 0.064 & \\
IOSUT & $15 / 29$ & $21 / 41$ & 0.002 & 0.967 & \\
IOFORAM & $0 / 38$ & $1 / 51$ & $0.000^{*}$ & $1.000^{*}$ & \\
PFORAM & $23 / 43$ & $37 / 52$ & 3.157 & 0.076 & \\
EPITER & $3 / 40$ & $6 / 52$ & $0.086^{*}$ & $0.770^{*}$ & \\
CORONL & $3 / 42$ & $2 / 53$ & $0.072^{*}$ & $0.789^{*}$ & \\
LAMBOSS & $18 / 48$ & $21 / 54$ & 0.021 & 0.885 \\
ASTRINB & $5 / 44$ & $1 / 53$ & $2.267^{*}$ & $0.132^{*}$ & \\
OMSUTS & $6 / 45$ & $2 / 51$ & $1.677^{*}$ & $0.195^{*}$ & \\
PARTNOTB & $8 / 40$ & $6 / 52$ & 1.255 & 0.263 & \\
CONDCANABS & $14 / 33$ & $28 / 44$ & 3.422 & 0.064 & \\
DIHYPOC & $4 / 40$ & $4 / 51$ & $0.000^{*}$ & $1.000^{*}$ &
\end{tabular}


Table 4.17. Continued

\begin{tabular}{|c|c|c|c|c|c|}
\hline Variable & Pre-2000 BP & Post-2000 BP & $x^{2}$ & p & Phi \\
\hline \multicolumn{6}{|l|}{ BILATERAL } \\
\hline TYMPDIHS & $20 / 41$ & $15 / 53$ & 4.148 & 0.042 & -0.21 \\
\hline FRSPINI & $15 / 35$ & $18 / 50$ & 0.408 & 0.532 & \\
\hline FOROVLI & $0 / 40$ & $2 / 49$ & $0.329^{*}$ & $0.566^{*}$ & \\
\hline PTSPBRTR & $5 / 37$ & $9 / 48$ & 0.416 & 0.519 & \\
\hline PTSPBRPRT & $1 / 37$ & $2 / 48$ & $0.000^{*}$ & $1.000^{*}$ & \\
\hline PTSPBRCOM & $1 / 37$ & $0 / 48$ & $0.017^{*}$ & $0.896^{*}$ & \\
\hline PTSPBRALL & $7 / 37$ & $11 / 48$ & 0.2 & 0.655 & \\
\hline PTABRGTR & $7 / 40$ & $19 / 51$ & 4.284 & 0.038 & 0.217 \\
\hline PTABRGPRT & $3 / 40$ & $8 / 51$ & $0.748^{*}$ & $0.387^{*}$ & \\
\hline PTABRGCOM & $1 / 40$ & $0 / 51$ & $0.015^{*}$ & $0.903^{*}$ & \\
\hline PTABRGALL & $11 / 40$ & $26 / 51$ & 5.123 & 0.024 & 0.237 \\
\hline AUDEXOS & $0 / 41$ & $0 / 53$ & $x$ & $x$ & \\
\hline MASTFRTEM & $26 / 40$ & $34 / 53$ & 0.007 & 0.932 & \\
\hline MASTFRSUT & $15 / 40$ & $16 / 53$ & 0.548 & 0.459 & \\
\hline MASTFROCC & $12 / 40$ & $19 / 53$ & 0.351 & 0.554 & \\
\hline ZYFFOR & $35 / 43$ & $41 / 51$ & 0.015 & 0.902 & \\
\hline MENTFORABS & $2 / 54$ & $0 / 47$ & $0.380^{*}$ & $0.537^{*}$ & \\
\hline ACCMENTFOR & $7 / 54$ & $5 / 47$ & 0.13 & 0.711 & \\
\hline MYLHBRD & $26 / 50$ & $26 / 45$ & 0.319 & 0.572 & \\
\hline MANDTOR & $0 / 56$ & $0 / 48$ & $x$ & $x$ & \\
\hline FRONTLN & $29 / 49$ & $30 / 54$ & 0.138 & 0.71 & \\
\hline FRONFOR & $15 / 43$ & $28 / 52$ & 3.416 & 0.065 & \\
\hline TROCSPR & $8 / 41$ & $3 / 52$ & $2.938^{*}$ & $0.086^{*}$ & \\
\hline OSJAP & $1 / 34$ & $12 / 45$ & 7.93 & 0.005 & 0.317 \\
\hline PARPROC & $1 / 44$ & $4 / 52$ & $0.533^{*}$ & $0.466^{*}$ & \\
\hline MARGFOR & $2 / 43$ & $1 / 50$ & $0.018^{*}$ & $0.894^{*}$ & \\
\hline SPBASBRTR & $8 / 40$ & $13 / 49$ & 0.521 & 0.47 & \\
\hline SPBASBRPRT & $6 / 40$ & $9 / 49$ & 0.178 & $x$ & \\
\hline SPBASBRCOM & $2 / 40$ & $4 / 49$ & $0.028^{*}$ & $0.867^{*}$ & \\
\hline SPBASBRALL & $16 / 40$ & $25 / 49$ & 1.077 & 0.299 & \\
\hline \multicolumn{6}{|l|}{ MIDLINE } \\
\hline METOPOPART & $2 / 24$ & $7 / 27$ & $1.631^{*}$ & $0.202^{*}$ & \\
\hline METOPFULL & $1 / 25$ & $1 / 27$ & $0.000^{*}$ & $1.000^{*}$ & \\
\hline BRAGMATB & $1 / 24$ & $0 / 27$ & $0.004^{*}$ & $0.953^{*}$ & \\
\hline SAGITOSSIC & $0 / 24$ & $0 / 27$ & $\mathrm{x}$ & $\mathrm{x}$ & \\
\hline APICALBN & $1 / 23$ & $3 / 27$ & $0.126^{*}$ & $0.722^{*}$ & \\
\hline INCABONE & $0 / 24$ & $0 / 27$ & $\mathrm{x}$ & $\mathrm{x}$ & \\
\hline PALTORUS & $1 / 22$ & $3 / 27$ & $0.096^{*}$ & $0.756^{*}$ & \\
\hline APICOSS & $2 / 19$ & $5 / 26$ & $0.144^{*}$ & $0.704^{*}$ & \\
\hline
\end{tabular}

* expected cell count in $2 \times 2$ table $<5$ so Continuity Correction values are used 
Figure 4.37. Distribution of the os japonicum discrete trait in Eastern Cape sample. Individuals displaying this discrete trait indicated by ellipse. ( $+=$ Pre-2000 BP, o $=$ Post-200 BP)

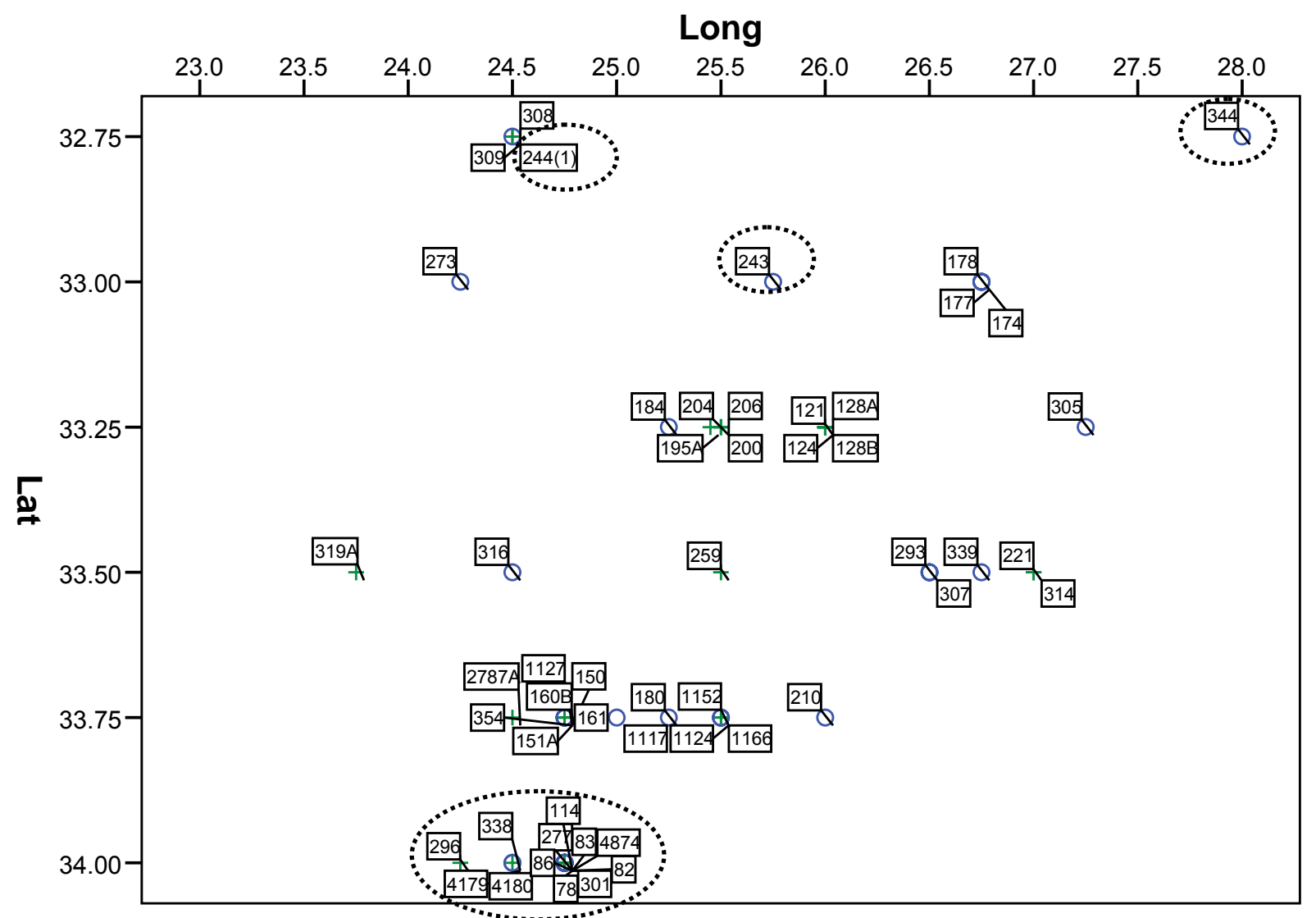

Francis area. The three outliers (ALB 244(1), 243, and 344) are all buried inland a considerable distance away from the coast and have post-2000 BP dates. It is possible that the grouping of individuals displaying this trait represents related kin, as the single pre-2000 BP individual with the os japonicum trait is also located in the St. Francis grouping. This finding can be interpreted as a reflection of genetic homogeneity of South African LSA groups. However, the fact that this sort of temporal and spatial patterning is observed in only one non-metric trait calls this interpretation into question.

In sum, sex and temporal differences in cranial non-metric trait frequencies are negligible for the Eastern Cape sample. These results support the overall findings of the 
metric skeletal analyses indicating that genetic homogeneity was maintained in the Eastern Cape region of South Africa.

\subsubsection{Comparisons with Other South African Samples}

The Eastern Cape sample is divided into pre- and post-2000 BP sub-groupings and frequencies for seven cranial non-metric traits are compared with four pooled-sex South African Bantu-speaking samples (Zulu, Xhosa, Sotho and Venda). The post-2000 BP subsample differs from all four Bantu-speaking samples for three cranial non-metric traits: tympanic dehiscence, mylohyoid bridge, and os japonicum (Table 4.18). Significant differences in the frequencies of the supraorbital foramen, tympanic dehiscence, and mylohyoid bridge are observed between the pre-2000 BP sub-group and the comparative Zulu, Xhosa and Venda samples. Frequencies for the pre-2000 BP sub-group differ from the Sotho sample for only tympanic dehiscence and mylohyoid bridge. Significant differences in the number of traits exhibiting significantly different frequencies and the distribution of the traits themselves are not observed between the between the pre- and post-2000 BP subgroups and the comparative Bantu-speaking samples. This suggests a high degree of genetic homogeneity in the Eastern Cape sample. The results of the comparative cranial non-metric analysis support the overall findings of this study. 
Table 4.18. Results of Pearson chi-square analysis of Eastern Cape and comparative South African Bantu-speaking cranial discrete trait frequencies

\begin{tabular}{|c|c|c|c|c|c|c|c|c|}
\hline \multirow{3}{*}{ Trait } & \multicolumn{8}{|c|}{ PRE-2000 BP (M and F combined) } \\
\hline & \multicolumn{2}{|c|}{$\begin{array}{l}\text { Zulu }^{1} \text { vs. Pre- } \\
2000 \text { BP }\end{array}$} & \multicolumn{2}{|c|}{$\begin{array}{c}\text { Xhosa }^{1} \text { vs. Pre- } \\
2000 \text { BP }\end{array}$} & \multicolumn{2}{|c|}{$\begin{array}{c}\text { Sotho }^{1} \text { vs. Pre- } \\
2000 \text { BP }\end{array}$} & \multicolumn{2}{|c|}{$\begin{array}{c}\text { Venda }^{1} \text { vs. Pre- } \\
2000 \text { BP }\end{array}$} \\
\hline & $x^{2}$ & $\mathbf{p}$ & $x^{2}$ & $\mathbf{p}$ & $x^{2}$ & $\mathbf{p}$ & $x^{2}$ & $\mathbf{p}$ \\
\hline Supraorbital Foramen & 6.997 & 0.010 & 6.611 & 0.025 & 2.4 & 0.200 & 4.098 & 0.050 \\
\hline Epiteric Bone & 0.097 & 1.000 & 0.111 & 1.000 & 0.0153 & 1.000 & 0.0389 & 1.000 \\
\hline Parietal Notch Bone & 1.493 & 1.000 & 1.224 & 1.000 & 1.342 & 1.000 & 0.14 & 1.000 \\
\hline Tympanic Dehiscence & 52.373 & 0.001 & 37.001 & 0.001 & 31.404 & 0.001 & 25.688 & 0.001 \\
\hline Accessory Mental Foramen & 0.0512 & 1.000 & 1.629 & 1.000 & 1.745 & 0.200 & 0.747 & 1.000 \\
\hline Mylohyoid Bridge & 33.119 & 0.001 & 27.222 & 0.001 & 29.932 & 0.001 & 43.716 & 0.001 \\
\hline \multirow[t]{2}{*}{ Os Japonicum } & 2.4336 & 0.200 & 0.054 & 1.000 & 2.669 & 0.200 & 2.079 & 0.200 \\
\hline & \multicolumn{8}{|c|}{ POST-2000 BP (M and F combined) } \\
\hline \multirow[t]{2}{*}{ Trait } & \multicolumn{2}{|c|}{$\begin{array}{l}\text { Zulu }^{1} \text { vs. Post- } \\
2000 \text { BP }\end{array}$} & \multicolumn{2}{|c|}{$\begin{array}{c}\text { Xhosa }^{1} \text { vs. Post- } \\
2000 \text { BP }\end{array}$} & \multicolumn{2}{|c|}{$\begin{array}{c}\text { Sotho }^{1} \text { vs. Post- } \\
2000 \text { BP }\end{array}$} & \multicolumn{2}{|c|}{$\begin{array}{c}\text { Venda }^{1} \text { vs. Post- } \\
2000 \text { BP }\end{array}$} \\
\hline & $x^{2}$ & $\mathbf{p}$ & $x^{2}$ & $\mathbf{p}$ & $x^{2}$ & $\mathbf{p}$ & $x^{2}$ & $\mathbf{p}$ \\
\hline Supraorbital Foramen & 1.773 & 0.200 & 1.655 & 0.200 & 0.0559 & 1.000 & 0.636 & 1.000 \\
\hline Epiteric Bone & 1.398 & 1.000 & 0.1778 & 1.000 & 0.439 & 1.000 & 0.296 & 1.000 \\
\hline Parietal Notch Bone & 0.0069 & 1.000 & 0.0283 & 1.000 & 0.0416 & 1.000 & 0.745 & 1.000 \\
\hline Tympanic Dehiscence & 27.575 & 0.001 & 16.479 & 0.001 & 12.462 & 0.001 & 9.918 & 0.010 \\
\hline Accessory Mental Foramen & 0.371 & 1.000 & 0.659 & 1.000 & 2.604 & 0.200 & 0.213 & 1.000 \\
\hline Mylohyoid Bridge & 28.953 & 0.001 & 23.572 & 0.001 & 26.143 & 0.001 & 40.168 & 0.001 \\
\hline Os Japonicum & 24.148 & 0.001 & 19.286 & 0.001 & 26.341 & 0.001 & 20.841 & 0.001 \\
\hline
\end{tabular}

${ }^{1}$ Rightmire (1976) 


\section{Chapter 5 - Discussion and Conclusions}

The findings of this study provide important insights into questions concerning the effects of the intensification of foraging behaviour and the mechanisms responsible for the introduction of sheep herding to southernmost Africa. In particular, this research speaks to three issues that are central to further understanding of Later Stone Age inhabitants of the Eastern Cape region of South Africa. First, a decrease in overall body size is observed from 3500 to 2000 BP across the Eastern Cape sample, which corresponds to the period of forager intensification. The timing of this reduction in mean body size argues against the idea that the adoption of sheep herding was a driving factor behind the initial skeletal change. Second, around 2000 BP body size returns to pre3500 BP levels. Correspondence between the timing of the recovery of body size to previous levels and the purported introduction of sheep suggests that this subsistence change may have been adopted to mitigate the apparent population stress and resource scarcity. Third, significant differences have not been identified in skeletal shape and cranial discrete trait frequencies, indicating that no substantial amount of novel genetic material was introduced into the indigenous population. However, significant increases in body size within the past 500 years suggest the influx of new genes from migrant agriculturalists could have been responsible.

The results of this study support the hypothesis that an in-situ development of sheep herding by means of diffusion is the most likely mechanism through which sheep herding was introduced to the Eastern Cape region of South Africa. This study's results are inconsistent with the proposal that sheep herding and associated southern African herder populations represent the introduction of a new, genetically distinct population. 


\subsection{Intensification of Foraging Behaviour and Decline in Body Size}

One of the most striking findings of this study is the general decline in mean adult body size in the absence of changes in shape between 3500 BP and approximately 2000 BP. Stature reduction is coupled with size reduction in areas of the skeleton the growth of which are believed to be more buffered against environmental stress, namely the cranium and dentition. The timing of this decline corresponds with the evidence of population stress and the intensification of foraging behaviour across the southern region. The temporal control that the inclusion of radiocarbon dates provides has enabled the current study to investigate and appreciate fine grained patterns of skeletal change. The fact that this decrease in size is observed not only in the cranial and postcranial skeleton, but also in the dentition speaks to the severity and magnitude of this period of stress.

Reduction in body size has been attributed to a number of complex environmental factors, such as climate change, dietary change, nutritional insufficiency, population density, disease load, and population mortality, as well as genetic factors, including gene flow and admixture. Research has demonstrated an inverse relationship between population density and body size in animal species (cf. Damuth 1981; Murdoch 1994; Schmid et al. 2000). This correlation has recently been demonstrated to apply to human populations (Walker in press). In southernmost South Africa, archaeological, bioarchaeological and paleoenvironmental research suggests that demographic and environmental factors contributed to the intensification of forager subsistence strategies at around 3500 BP. During this time various lines of evidence suggest that population densities were greater along the southern coastal region than any previous time during the Holocene. Foraging groups inhabiting the Cape region at around 3500BP exhibited many 
of the hallmarks of a population intensifying their subsistence as a result of external stress. Evidence of stress both on human populations and the local resources is evident through apparent reduced human mobility, increased food storage and processing, and dietary shifts. Resource scarcity and imbalance associated with increased population growth sets up conditions suitable for the intensification of productivity, reduction in mobility, and an increase in territoriality (Price and Brown 1985). Evidence for increased territoriality and identity signalling, additional key characteristics of intensification, is demonstrated by the individuation of tool production at coastal sites in the Eastern Cape (Binneman 2004/2005). Other aspects of intensification, namely increases in the diversity and specialization of the diet, have been observed among Cape foragers. The establishment of food specialization and dietary niches has been noted at closely related sites in the southern Cape (Sealy 2006), along with a general shift towards a reliance on smaller food packages, mainly smaller terrestrial mammals and a focus on marine resources, with plant foods constituting a greater proportion of the diet (Parkington 1980, 1983). Changes in the form and incidence of rock art have also been interpreted as indicators of population stress. During the period of intensification, foraging groups were not only moving into the mountains to use previously unoccupied rock shelters (Manhire et al. 1984), but an increase in the incidence of rock art in these shelters has been observed as well (Parkington et al. 1985).

During the mid to late Holocene South African foraging populations may not have been as isolated as during early Holocene times when the interior region of South Africa was abandoned. The relocation of interior foraging groups to the Cape coastal regions between $8000 \mathrm{BP}$ and $4000 \mathrm{BP}$ in response to a cooling and drying trend that altered the 
local floral and fauna, making the interior region largely uninhabitable, served to somewhat isolate LSA foragers from other groups living in the north. The increase in population density that eventually resulted from this wide scale relocation is cited as one of the main factors that contributed to the intensification of foraging behaviour across the Cape region after 4000 BP. However, evidence of contact and trade among foragers across the southern African region exists throughout the LSA. The variability and range of exotic goods discovered at sites located great distances from the items' origins attest to the extensiveness of the exchange networks that were maintained during the Holocene. The maintenance of extensive interaction networks among foraging groups helped to facilitate cultural continuity and would also have been the catalyst that enabled the transport of sheep to groups in the south. It can be argued that it may have been impossible to maintain systems of reciprocity and exchange, such as hxaro, during times of widespread resource scarcity. The hxaro system, which was intended to alleviate localized resource stress by widening one's access to land and resources through affiliation with an individual in another foraging group (Smith 1994; Binneman and Hall 1987; Hall 1990), may not have been sustainable during the period of intensification when resource stress may have been extensive across the southern region and evidence suggests that foragers were becoming more territorial.

The timing and characteristics of the temporary reduction in body size observed among the LSA inhabitants of the Eastern Cape suggest that the intensification of foraging behaviour at around $3500 \mathrm{BP}$ may have served as the proximate cause. Differences in cranial size occurred, but they are slight and they pertain mainly to the facial region. The postcranial skeleton, in comparison, exhibits more marked size 
changes in the absence of evidence of significant shape change. This pattern of size change conforms to current understandings of skeletal growth, development, and plasticity. Regions of the cranium develop at different rates and follow different growth trajectories. The growth and development of the cranial vault tracks that of the brain, while the face and mandible follow the postcranial pattern (Sinclair and Dangerfield 1998). The growth of the brain and supporting skeletal tissue occurs rapidly and takes place very early in life, approaching adult size around the second year of life (Sinclair and Dangerfield 1998). The vault and basilar regions are more buffered against external stress than the face, mandible and postcranial skeleton, since the neurocranial region protects both genetic information and the biological blueprint necessary for the optimal functioning of the organism (Wood and Liberman 2001). In comparison, the growth of the face, mandible and postcranial skeleton follows a moderate pace through childhood, increasing significantly during puberty. This extended period of skeletal growth means that there is a greater potential for adverse environmental factors to affect the development of these regions, as compared to the cranial vault region.

Different patterns of cranial size between the sexes are perplexing. The most striking decreases in size post 3500 BP pertain to male faces. Female facial size appears to remain stable through time. No significant changes in the size of the vault region or mandible were observed, yet vault size became more variable in both sexes between 3000 to $2000 \mathrm{BP}$. The absence of any significant size changes in the mandible is somewhat unexpected. In light of the differing developmental trajectories of the cranial region, changes in mandibular size would be expected to accompany facial size changes. Instead, it appears that variability in mandibular size decreases during the presumed 
period of intensification. This pattern is also unanticipated considering that stress tends to be reflected as an increase in size variability (Pfeiffer and Sealy 2006).

The observed changes in cranial shape are minor and sex specific, and are thus difficult to interpret. A change in the shape of the male vault with breadth decreasing while length increases, has been observed through time. The statistically significant, yet weak, change in male vault shape is perplexing given the demonstrated relative stability of the vault region. Although the second principal component is generally interpreted to reflect shape, it is possible that other subtle yet complex factors contribute to this slight shape change. Female mandibles also exhibit slight changes in shape as width increases and length and height decrease through time. Some change in the shape of the facial region is observed between 3500 and $2000 \mathrm{BP}$, especially in females, but this is not statistically significant. It is not unexpected that minor changes in facial shape might accompany changes in facial size, given that this region of the cranium demonstrates considerable plasticity. Because the mandible is more directly involved in the movements and forces of mastication, the observed stability in mandibular size, in conjunction with minor changes in form, may serve as an indication that even thought the diet may have changed somewhat, the broad dietary components and mastication activities were maintained. The patterns of cranial size and shape identified in the current study are corroborated by the research of Stynder (2006) on a large sample of South African cranial specimens spanning the late Pleistocene to the late Holocene, and together support a general phenomenon of population stress across the Cape region. Stynder (2006), like the current study, observed a decrease in cranial size and only minor changes in craniofacial shape pertaining only to the facial and frontal regions (Stynder et 
al 2007). Although the focus on smaller and different food packages, coupled with environmental changes, could have been sufficient to temporarily and slightly alter the size and form of the facial and mandibular regions, based on the developmental relationship with the postcranial skeleton, it seems most probable that these face and mandible changes are simply varying with stature and postcranial size. It is possible that other variables, like changes in the division of labour or the use of the mouth for tools, may also be relevant.

The presence of a general pattern of short term reduction in dental size is somewhat unexpected given that the dentition is believed to be more genetically controlled (Kieser 1990) and not as influenced by environmental stressors as skeletal tissue. However, the decline in tooth size observed throughout the dentition between approximately 3500 and $2000 \mathrm{BP}$ is consistent with short term population stress and food scarcity. Changes in tooth size have been identified among genetically homogeneous populations and have been attributed to modifications to the local environment, specifically changes in the quality of the diet (Garn et al. 1968). Research has demonstrated that negative environmental factors, such as nutritional stress, can produce a reduction in dental size (Larsen 1997; Stojanowski 2005; Stojanowski et al. 2007). The LSA forager maxillary anterior teeth exhibit a significant drop in tooth size, coupled with an increase in variability, between 3500 and 2000 BP. The maxillary posterior teeth and the mandibular anterior teeth also exhibit a size decrease, with values completely outside the pre-3500 BP range. In comparison, while the mandibular posterior teeth also undergo a significant, short-lived size decrease, a few individuals display teeth that fall within the lower limits of the pre-3500 BP dental size range. Interestingly, individuals 
with the smallest teeth date between 2500 and 2000BP, when it is expected that population stresses were greatest. This pattern extends to all teeth except the maxillary anterior teeth.

Relationships between tooth size reduction and changes in dietary composition have also been explored in the bioarchaeology literature. Large teeth are believed to be advantageous in situations where tough and gritty diets and the use of the teeth as tools produce high rates of tooth wear because their large size should act to protect against the adverse effects of extreme wear (pulp exposure, abscesses and tooth loss). Consequently, small teeth should be selected for in cases where tooth wear is reduced through subsistence innovation involving increased food processing (Sciulli 1997). LSA South African foragers exhibit substantial dental wear (Sealy et al. 1992; Pfeiffer 2007). However, no temporal changes in the rate of tooth wear, or the frequencies of caries, antemortem tooth loss, or dental abscesses, have been observed among the LSA inhabitants of the Eastern Cape that appear to correlate with subsistence change (Ginter 2005). This suggests that broader factors associated with changing subsistence behaviours, beyond the actual dietary components, may be responsible for the apparent reduction in tooth size.

Significant short term decreases in skeletal size between 3500 and 2000 BP are observed in the postcranial region in the absence of shape change. The marked decrease in postcranial linear dimensions is consistent with the differential growth produced by dietary insufficiency (Sinclair and Dangerfield 1998). Males display a somewhat more significant reduction in trunk size during this period, as compared to females. Similar to the pattern observed in the dentition, the smallest individuals date between 2500 to 2000 
$\mathrm{BP}$, the time just prior to the introduction of sheep when stress may have been the most significant. The upper and lower limbs display decreases in linear dimensions of similar magnitude for males and females, and there is an increase in variability. Although this period sees individuals with shorter limbs than any other time during the Holocene, limb sizes for a number of individuals fall within the range observed prior to $3500 \mathrm{BP}$. Variability in limb size during this period, with some individuals continuing to display limb lengths in the pre-3500 BP range, suggests that population pressures and nutritional stress may have impacted the population differently. It follows then that because small body size would be profitable during a time of resource scarcity, genetically smaller individuals who required fewer calories for survival may have been more likely to survive to adulthood this period of stress intensification.

\subsection{Was sheep herding adopted to alleviate population stress?}

"Stress in human adaptation is resolved by changes in human behaviour."

(Price and Brown 1985:10)

The adoption of innovation is advantageous during times of population pressures and resource scarcity because it can be a means of reducing stress on the population and resources (Shennan 2001). The results of this study support the hypothesis of an in-situ development of sheep herding in the Eastern Cape region. The return of body size to pre-intensification levels around 2000BP and the general homogeneity in cranial discrete trait frequencies (see below, 5.2.3) suggest that the introduction of sheep and the herding lifestyle by another population, even a closely related one, is not probable. The findings of this study do not support the suggestion that the arrival of sheep herding served as the stimulus for the social and economic change observed in southernmost Africa. It seems 
more probable that a combination of factors relating to population stress and climatic change brought about the need for foraging groups to diversify their subsistence. The adoption of herding may have offered a solution to the problem of nutritional insufficiency and food scarcity as manifest in reduced body size. If sheep had been introduced to the Cape region via the migration of a genetically and culturally similar group, some differences in skeletal and dental characteristics beyond those that have been identified in this study would be apparent.

\subsubsection{Skeletal evidence of diffusion}

After 2000 BP a return of body size to that observed prior to the intensification of foraging behaviour is observed in the cranium, dentition, and postcranial skeleton. The recovery of body size after the temporary reduction during the probable period of stress between 3500 and $2000 \mathrm{BP}$ supports the proposition that the adoption of a new subsistence behaviour in sheep herding may have been undertaken as a way to alleviate population stress among both the people who adopted it and those who continued in the foraging tradition. Around $2000 \mathrm{BP}$ cranial size returns to pre-3500 BP levels with most data points falling within the pre-3500 BP range. While a considerable amount of variability in cranial size is observed after $2000 \mathrm{BP}$, individuals with small vaults of the magnitude observed between 3500 and 2000 BP are not present after approximately 1800 BP. Facial size becomes more variable, especially among females, with the addition of individuals with larger faces after 2000 BP. This pattern of cranial size change can be interpreted as a reflection of the diversification of subsistence practices with the advent of sheep herding in conjunction with a greater availability of foodstuffs. Stynder and colleagues (2007) also observed a rebound in the size of their South African cranial 
sample to pre-stress levels, which they attribute to the ability of the skeleton to respond to rapid environmental change as a result of its plasticity.

Increases in size are also present across the dentition around $2000 \mathrm{BP}$, but unlike the cranium, the mean and range of the pre-3500 BP sizes are not repeated. Overall dental size rebounds at around $2000 \mathrm{BP}$, but this is restricted to the mid to lower pre-3500 BP range. This partial recovery of tooth size may be associated with the generalized trend in tooth reduction among most human populations from that of our early modern human ancestors possibly stemming from developments in food production, reduced reliance on the teeth for processing with the development of complex lithic technology, and dietary change (c.f. Kieser 1990; Brace et al. 1991). While dental development is more cushioned against environmental stress than other parts of the skeleton, improvements in one's environment have been observed to positively affect dental development. Secular changes in tooth size have been observed within a particular population. Increases in tooth size have been documented over a short period of time among the offspring of immigrant groups and between different generations paralleling increases in food availability and quality (Harris et al. 2001). However, it seems that other environmental changes, beyond improvements in dietary quality, may be responsible for increases in dental size. Although a positive odontometric trend has been observed in a study of the dentition of urbanizing black populations, the quality of the urbanized diet was shown to be inadequate in that instance (Keiser et al. 1987). The general increase in size throughout the dentition around 2000 BP may be linked to the alleviation of socioeconomic pressures existing during the prior period. Dietary change associated with the introduction of sheep may have been only one factor contributing to 
this physiological improvement. Climatic modifications, and changes to the distribution of foragers across the landscape could also have served to ease the strain of the preceding period.

Postcranial size begins to rebound to pre-3500 BP levels at around $2000 \mathrm{BP}$, with a magnitude of recovery that is more striking than that seen in other anatomical regions. Just prior to $2000 \mathrm{BP}$ individuals exhibit the smallest trunks and shortest limbs of any other period throughout the Holocene, suggesting that competition for resources was greatest and nutritional quality lowest during this time. The trunk and limbs continue to increase in length after $2000 \mathrm{BP}$, with the most dramatic increase in size and variability occurring around $500 \mathrm{BP}$. The increase in upper and lower limb length appears to be more extensive and rapid than in the trunk. After 2000 BP upper limb linear dimensions appear to be less variable than that observed prior to the period of intensification. This is puzzling considering that increased variability should be expected to accompany the diversification of subsistence behaviours, especially in a situation such as this where a new food source is added to, but does not replace the traditional subsistence economy. Addressing the linear increases in both upper and lower limbs, milk has been suggested to have a significant impact on growth. A stable food source that sheep milk would provide to the diet could contribute positively to body size considering that it is high in both protein and fat, components that were likely lacking in the forager diet. Research that has studied the influence of milk on growth potential suggests that milk supplementation has the most impact on the growth of undernourished children (Wiley 2005). It can be argued, based on the various lines of evidence, that between 3500 and 2000 BP LSA foragers were undernourished. The magnitude of the body size rebound at 
around 2000 BP could possibly be attributed to the introduction of a more stable food source such as sheep’s milk. However, the strength of the association between skeletal change and the advent of sheep herding must be qualified as it cannot be assumed that the initial introduction of sheep and herding was pervasive and immediate. Although there is evidence for sheep in the area by around 2000 BP, it is not likely that this novel food source is exclusively responsible for the alleviation of the apparent nutritional stress. At the sites across the Cape with the earliest archaeological signatures of sheep, sheep remains constitute very minor components of the faunal assemblages, suggesting that the contribution of this resource to the subsistence economy would not have been significant (Sadr 1998). Other factors that appear to have adversely affected foraging populations seem to have improved as well. A return to slightly warmer and drier conditions around 2000 BP coupled with evidence of a redistribution of populations away from open coastal sites in favour of a reoccupation of rock shelters and inland locales (Henshilwood and Parkington 1994; Jerardino et al. 2008) may have also helped to ease the effects of resource stress.

As reviewed in Chapter 2, the distribution of radiocarbon dates from sites with domestic stock supports Elphick’s (1977) proposal concerning the southward movement of sheep from the Zambezi region to the Cape. However, Smith (1992) suggests that sheep only became a significant presence in western and southern Capes around $1600 \mathrm{BP}$, calling into question the suggestions about the impact of sheep and herding on existing foragers that have been offered based on the Eastern Cape skeletal evidence. It is possible that small scale migrations of foragers-turned-herders from northern Botswana could have introduced sheep to the Cape region. The timing of the Bantu migration, 
coupled with the distribution of dated sheep remains and Iron Age artifacts in forager contexts in the Zambezi region indicates that some degree of contact occurred between foraging groups in northern Botswana and early Iron Age agriculturalists that for a time settled there. It is probable that after a period of contact involving an exchange of objects and ideas that some foragers may have adopted sheep. The tumultuous period of Early Iron Age migration and resettlement in the Zambezi region may have prompted some foraging groups with sheep to move south in search of available grazing land. However, as the above discussion argues, the movement of peoples was not necessary for the introduction of sheep. Rather, it could be that extensive trade networks, such as the hxaro system, would have been sufficient to transport sheep across this region.

Comparisons between the Eastern Cape and other South African samples and the findings of the cranial discrete analysis reinforce the suggestion that an in-situ development is the most likely scenario concerning the introduction of sheep herding in southernmost Africa.

\subsubsection{Insights from the comparative analyses}

The results of the comparative skeletal analysis generally favour the hypothesis of indigenous development of sheep herding rather than a foreign introduction. Not surprisingly, the cranial morphology of the Eastern Cape sample is more similar to protohistoric foraging and herding groups from the Orange River area and historic Khoesan, than to the African Bantu-speaking samples. The magnitude of differences between the post-2000 BP subsample and the comparative Bantu-speakers and protohistoric foragers and herders is less than for the pre-2000 BP subsample. The degree of cranial congruence between the pre-2000 BP Eastern Cape males and the South 
African Khoesan and Reit River male samples is as expected. However, with differences restricted only to orbital height, similarities with the Abrahamsdam males are intriguing because this sample has been identified ethnohistorically and archaeologically as protohistoric pastoralists (Morris 1992). This established relationship between the Eastern Cape and Abrahamsdam males suggests that differences in cranial size between foragers/probable pastoralists and established pastoralists are not substantial, providing further support to the craniometric multivariate results and the close relationship between foragers and pastoralists.

The incongruity in cranial size between the pre-2000 BP females and the Bantuspeaking groups (West-Central African, Central African Bantu, East-African and South African Bantu) is expected. One would expect that the pre-2000 BP group, consisting exclusively of female foragers, would exhibit a high degree of similarity to the South African Khoesan (Ribot 2003) and Riet River (Morris 1992) samples, considering that both consist of foragers of the same lineage. Given the expected relationship among these three groups, the number of craniometric variables that differ significantly ( $\mathrm{n}=5$ and 4, respectively) is rather high. Temporal differences between the Eastern Cape and the two comparative foraging samples (both date to a more recent period) may explain some part of this incongruity. When time period and context from which these two comparative foraging samples originate are considered, the magnitude of difference appears probable.

The comparative odontometric results support the diffusion hypothesis for the establishment of the herding lifestyle in southern Africa. Similarities between the dental dimensions of the Eastern Cape and contemporaneous LSA samples from the Southern 
and Western Capes are as expected. In comparison, the Eastern Cape sample exhibits larger teeth in the buccolingual and mesiodistal dimensions than the modern San sample. This finding is in keeping with the generalized reduction in tooth size through time, yet other factors may also contribute. As modern foragers, the San have experienced a long history of environmental change and population contacts. Contact with both agricultural and European immigrants likely affected aspects of their foraging life, including diet, food preparation techniques, and settlement patterns. Yet, it is interesting that these factors did not produce increases in tooth size, as has been observed in other situations. Furthermore, considering that congruity in body size has been demonstrated between South African LSA foragers and modern San groups (Kurki et al. 2007), this correlation could be expected to extend to the dentition as well. Some differences were identified between odontometric dimensions for the Eastern Cape and Griqua samples, but the magnitude of difference with the modern South African Bantu-speaking sample was much greater. The affinities of the Griqua groups with ancestral Khoesan and historic Iron Age groups, as supported by Morris’ (1992) craniometric findings, help to account for the relationship between the Eastern Cape sample. The prevalence of significant differences between the Eastern Cape and Bantu-speaking odontometric values supports the idea of genetic continuity in the Eastern Cape sample through time.

The results of the comparative postcranial metric analysis, although restricted to only South African Bantu-speaking skeletal samples, support the broader findings of this research. The pre-2000 BP postcranial dimensions differ significantly from the comparative data for both males and females. In comparison, the post-2000 BP Eastern Cape males and females exhibit some similarities in limb dimensions with the Bantu- 
speaking samples. The significant size differences between the small bodied pre-2000 BP Eastern Cape sample and the large bodied Bantu-speaking samples is likely enhanced by the period of body size decrease observed between 3500 and 2000 BP. Similarly, the presence of some very large Eastern Cape individuals who date to the last 500 years has possibly contributed to the body size similarities with the Bantu-speaking samples.

\subsubsection{Cranial discrete trait frequencies and the diffusion hypothesis}

The nature and patterning of the observed modifications to physique suggest that sheep herding was not introduced by a genetically distinct population. However, it is the results of the cranial discrete trait analysis that provide the strongest support for the diffusion hypothesis. Even though continuity of the foraging lifestyle has been demonstrated among prehistoric foraging groups living throughout southern Africa, regional variations in climate and environment exist. Human skeletal morphology (size and shape) can be different between populations living in different environments, even if they share basically similar lifestyles. Because of this, researchers attempt to identify subtle genetic differences between neighbouring populations by examining skeletal discrete trait frequencies (cf. de Villiers 1967; Molto 1983; Cybulski 1992; Prowse and Lovell 1996; De Laurier and Spence 2003). Therefore, discrete traits should be able provide insights into whether is it probable that sheep were introduced to southernmost Africa by distinct, yet genetically similar groups originating in northern Botswana.

The current study found that there were virtually no differences in cranial discrete frequencies between the sexes or across time and space. Frequencies differed significantly between the sexes for only two traits, partial pterygoalar bridge (18\% males, $4 \%$ females) and apical ossification (29\% males, $4 \%$ females), with males exhibiting a 
higher incidence of traits in both cases. Given that fifty-two pairwise comparisons were undertaken, it may be expected that a small number of significant differences may occur on the basis of chance, even if the samples are drawn from the same population. In keeping with the ideas proposed by Spence (1974), the high degree of similarity in discrete traits across time in the Eastern Cape sample represents low variability in the sample under study. Further, the absence of so few significant differences in trait frequencies between the sexes reflects similarity in the degree of relatedness between males and females. If patrilocal or virilocal residence was practiced by LSA groups, a higher level of variability in trait frequencies between the sexes could be expected (Spence 1974). However, such a pattern is not observed among the Eastern Cape sample. The high degree of homogeneity between the sexes in cranial discrete trait frequencies suggests that Southern African foragers sought mating partners from neighbouring foraging groups, thus maintaining continuity among South African foraging groups through time. Had even small groups of foragers-turned-herders migrated south with sheep around $2000 \mathrm{BP}$, it could have been reflected in the frequencies of some discrete traits, if the study sample included crania representing the new immigrants or their offspring. There is no such evidence.

While only four out of the fifty-two discrete traits examined yielded significant differences across time, a strong statistical difference between pre-2000 BP and post2000 BP individuals was only produced for os japonicum (3\% vs. 27\%, respectively). The distribution of the individuals exhibiting this trait is of particular interest. Ten out of the thirteen individuals displaying this trait are buried at the coast in the Cape St. Francis area. Included in this group is the only pre-2000 BP individual who possesses the os 
japonicum trait. The remaining three individuals displaying this trait are each interred in different inland burials located a considerable distance from the coast. The inclusion of radiocarbon dates as a variable of analysis adds further meaning to this cranial discrete trait pattern. In the absence of radiocarbon dates, the patterning of this trait across the Eastern Cape landscape could be interpreted to support the migration hypothesis because the distribution of individuals with this trait loosely conforms to the north-south migration route of herders with sheep proposed by Elphick. However, the fact that the individual exhibiting the os japonicum trait with the oldest ${ }^{14} \mathrm{C}$ date is part of the coastal concentration suggests that this trait emerged as an indigenous evolution, rather than an outside introduction. This interesting pattern, along with the lack of substantial temporal differences in trait frequencies between the pre- and post-2000 BP subgroups argues against the possibility that different, but closely related groups from the north could be responsible for the introduction of sheep herding. It appears that for some reason this trait in the absence of others was passed along family lines, suggesting that most of the individuals in this kin group inhabited a small area of the coast after $2000 \mathrm{BP}$, which could possibly be interpreted to indicate the restriction of territories or the maintenance of closer connections with the landscape. Yet, the occurrence of this trait in three individuals buried a considerable distance inland from the larger concentration argues against this possibility, and instead supports the continuation of extensive mobility and connections between neigbouring foraging groups. Given the landmass that is encompassed by the study area and the relatively small numbers of prehistoric skeletal remains that have been recovered, it is remarkable to have identified a discrete trait with such a strong temporal and spatial pattern. 
The results of the comparative analysis corroborate this assertion and add further support to the argument that herding was not introduced by a migration. Both the pre2000 BP and post-2000 BP subgroups differ significantly from the four South African Bantu-speaking samples for two out of the seven cranial discrete traits compared (mylohyoid bridge and tympanic dehiscence). Supraorbital foramen frequencies differ significantly only between the pre-2000 BP subgroup and the comparative group. Significant differences in the frequency of the os japonicum trait are only observed between the post-2000 BP subgroup and the comparative samples. While some differences in trait frequencies are observed between the Eastern Cape subgroups and the comparative samples, these findings are consistent with the broader cranial discrete trait results. Unfortunately, the limited availability of comparative data permits comparisons to be made with only seven of the traits observed in the current study.

Of the traits compared, the differences in os japonicum trait frequencies between the Eastern Cape subsamples and the Bantu-speaking samples are the most important to the findings of this study. Significant differences in the frequency of this trait are not observed between the pre-2000 BP subsample and the comparative samples, yet differences are significant between the post-2000 BP and the comparative samples. The four Bantu-speaking samples selected for comparison are ancestral to the Iron Age people who migrated out of West Africa and settled in the Zambezi valley region and subsequently in the eastern part of South Africa, making them genetically different from the indigenous foragers and herders who occupied southernmost Africa prior to their arrival. This pattern of difference in the frequency of the os japonicum trait between the post-2000 BP subgroup and the comparative Bantu-speaking samples reinforces the 
assertions that were made based on the distribution of the trait in the Eastern Cape study sample. If this trait had somehow appeared in the Eastern Cape sample via contact with another group, it could be seen in other groups who the Eastern Cape foragers or members of their extensive trade network would have encountered. It seems probable, therefore, that the presence, as well as the spatial and temporal distribution, of the os japonicum trait in the Eastern Cape sample reflects a limited kin relationship.

\subsubsection{Changes in gene flow and the diffusion hypothesis}

Prior to 2000 BP, the results of the Relethford-Blangero analysis indicate genetic isolation of the people of the Eastern Cape. This analysis also indicates an increase in gene flow from a foreign source after $2000 \mathrm{BP}$. The latter result appears to support the migration hypothesis for the introduction of sheep herding to southernmost Africa.

Significant differences in the direction and magnitude of the pre-2000 BP and post-2000 BP residual values indicate that the genetic structure of the Eastern Cape population changed at about that point. A shift from high levels of genetic homogeneity prior to $2000 \mathrm{BP}$ to significant levels of genetic heterogeneity after $2000 \mathrm{BP}$ is evidenced by the significant negative pre-2000 BP and positive post-2000 BP residual values.

However, if sheep herding in southernmost Africa was introduced by the migration of foreign groups, other significant changes in skeletal morphology should also be evident. Only cranial size shows this pattern; cranial shape does not. Therefore, it is necessary to consider alternative interpretations and explanations for these findings. This involves considering the population genetic findings along with the other outcomes of this study, and situating them in the broader archaeological context of the Eastern Cape during the mid- to late Holocene. 
The significant residual values provide a solid case for the migration hypothesis. However, the absence of any significant change in skeletal morphology, as evidenced by the nonsignificant PCA results, contradict this interpretation. With the exception of an increase in cranial size through time, a pattern that also applies to the postcranial skeleton, no other changes are identified by the PCA of the cranial variables used in the Relethford-Blangero analysis. Because the observed changes in skeletal morphology are restricted to size and do not extend to shape, these changes are not consistent with the influx of new genes from a nonlocal source.

The results of the Relethford-Blangero analysis must also be considered in the context of the timing and nature of the skeletal size changes. The skeletal size changes are initiated long before the generally agreed upon date for the arrival of sheep, instead coinciding with the onset of the intensification of foraging behaviour that involved modifications to aspects of traditional foraging like increased territoriality, a reorientation of subsistence practices towards smaller food packages and a focus on marine resources, the initiation of food storage, and the emergence of markers of specific group identity. It is apparent, based on the recovery of body size, that this stress was somehow alleviated sometime around $2000 \mathrm{BP}$. It is possible that the introduction of sheep may have played some role in the alleviation of stress. Yet, the timing of this recovery is not sufficient to suggest migration because the nature of this body size recovery is in line with that observed prior to the period of intensification. Had the body size recovery consistently exceeded the apparent forager norm, as based on the pre-intensification levels, this could be considered as support for the migration hypothesis. 
When the population genetic analysis results are considered along with paleoenvironmental and archaeological information they support the diffusion hypothesis. The period between approximately 2000 to 3500 BP was a time of socioeconomic change placing stress on foraging groups inhabiting the coastal region of South Africa. Archaeological and skeletal evidence paint a picture of a less mobile and more territorial forager population that was feeling the combined effects of their increased population density and declining environmental conditions on both the amount and diversity of available food resources. Improvements to the forager situation coincide with the appearance of sheep in the Cape region around $2000 \mathrm{BP}$, as well as evidence of a return again to increased forager mobility. When situated in this context the less than expected pre-2000 BP and greater than expected post-2000 BP levels of gene flow can be adequately understood independent of major migration from outside the region. Concomitant with the suggestion of increased forager mobility is the likelihood that foragers from other parts of the South African Cape may have migrated into the Eastern Cape area after 2000BP, but were not present in the area prior to that time, and thus are not represented in the skeletal sample. Because the post-2000 BP sample is relatively small in number, the influx of biologically similar, yet nonlocal, foragers into the area could be expected to increase genetic variability.

It is also possible that a migration event that took place some time after $2000 \mathrm{BP}$ may be responsible for the increased levels of genetic heterogeneity observed for the post-2000 BP sample. Sadr (1998) and others have suggested that the Khoekhoe herding groups that the first European explorers encountered at the Cape may represent a later migration of pastoralists at around $1000 \mathrm{BP}$. As this study has demonstrated a significant 
change in skeletal size and shape is not detected at 2000BP, yet physical differences pertaining mainly to stature were noted between historic Khoekhoe herders and San foragers inhabiting the Cape region (Schapera 1930). Although Khoekhoe and San groups exhibited differences in stature and subsistence, cultural, linguistic and physical commonalities support an ancestral relationship. A scenario concerning a more recent migration of foragers turned pastoralists from northern Botswana who obtained sheep and possibly marriage partners from Bantu-speaking agro-pastoralists is supported by the population genetic, multivariate and cranial discrete findings of this study. Had a significant pastoral migration taken place at around $2000 \mathrm{BP}$, a sudden change in material culture and subsistence practices should be detected archaeologically, but as discussed in Chapter 2, this evidence is not present. The discovery of faint pastoral signatures at sites south of the Zambezi to the Cape region dated to around 2000 BP attest to a sheep presence. However, the lack of a significant component of domestic remains in faunal assemblages at these sites suggests that sheep herding may not have had a major impact on the subsistence economy of LSA groups inhabiting this region at around $2000 \mathrm{BP}$. The absence of a significant pastoral archaeological signature across the Cape region (Sadr 1998) coupled with a lack of isotopic evidence from skeletal remains from Western and Southern Capes for the dietary use of sheep (Pfeiffer and Sealy 2006) indicates that sheep did not form a significant component of the diet. The balance of the skeletal findings of this study and the archaeological evidence from the Cape region suggest that 'true' pastoralism was likely not practiced by some LSA groups in this region of the Cape in the centuries immediately following $2000 \mathrm{BP}$. 
In opposition to this interpretation of the population genetic analysis results, one could argue that the Eastern Cape sample under study is not composed exclusively of individuals from this period of intensification (3500 BP to $2000 \mathrm{BP}$ ) or the period immediately following the introduction of sheep (2000-1000 BP). However, as it turns out more than fifty percent of the individuals in the pre-2000 and post-2000 BP groups fall within this tumultuous period of the Holocene. This unequal distribution of individuals throughout the pre-2000 BP and post-2000 BP periods respectively, could also explain the consistency between the population genetic findings and the other lines of evidence. Furthermore, if social-cultural boundaries were eventually established between neighbouring herder and forager groups that restricted the exchange of marriage partners, as some researchers like Smith (1998) suggest, this could have contributed to the increase in genetic heterogeneity observed after 2000 BP. Regardless of the mode of transmission of this new way of life, foragers and herders did not interact in the same way as foraging groups had previously done, and this would have contributed to an increase in genetic heterogeneity in this region. It must be kept in mind that because of limitations of the skeletal sample and the current inability to clearly demarcate forager and herder occupations and interments, the post-2000 BP subpopulation comprises the remains for foragers and purported herders. In effect, the post-2000 BP subsample may be comprised of two 'populations': foragers and herders. If barriers to contact and gene flow did exist between these two subsistence populations, then any analysis that examined their genetic composition as a whole would detect evidence of greater heterogeneity, one interpretation of which could be increased extra-regional gene flow. 
In contemplating the implications of the population genetic analysis results on the broader findings of this study it is also necessary to consider the other compounding factors that may influence those results. The significant time depth and noncontemporaneity of the Eastern Cape skeletal sample may also pose a potential problem for the assumptions of the Relethford-Blangero model, and thus the accuracy of the results. As discussed in Chapter 3, certain assumptions about the relative genetic and environmental influences on quantitative phenotypic traits are made in order to justify their utility in population genetic questions. Population genetic models assume that the environmental influence on the quantitative traits in question will be the same across all populations (Williams-Blangero and Blangero 1990: 132). Satisfying this assumption has not been as issue for many of the studies that have utilized quantitative variables to examine questions of genetic variation and gene flow, because they have focused on skeletal samples that are generally contemporaneous (Williams-Blangero and Blangero 1989, 1990; Relethford and Blangero 1990; Relethford et al. 1997; Schillaci and Stojanowski 2005) or that span a relatively limited time frame (cf. Steadman 1999, 2001). The large temporal range of the Eastern Cape LSA sample under study, spanning approximately 4000 years, appears to violate this assumption, thus opening the possibility of unequal environmental variance in the craniometric traits across the subpopulations. In other words, the greater time depth of the Eastern Cape sample allows for the possibility that environmental influences may have contributed to the levels of variation observed, preventing the measures of heterogeneity from being interpreted in a purely genetic framework. The small size of the sample available for the population genetic analysis and the fact that the analysis is restricted to two 'populations' may also have 
effects on the results. Even though parameters are built into the Relethford-Blangero model to control for the potential effects of genetic drift in small populations, such as the scaled and unbiased $\mathrm{R}$ matrices, dealing with only two small subpopulations reduces the analytical strength of this approach.

\subsection{Variability in the timing of changes in body size across the Cape region}

While the general pattern of body size reduction during the period of apparent stress has been observed across the Cape region (Pfeiffer and Sealy 2006; Stynder 2006, Stynder et al 2007), the timing of the onset of the body size changes appears to be somewhat distinctive to the Eastern Cape. The findings of the current study suggest that Eastern Cape foraging groups began to experience the effects of population and resource stress, as manifest through retardation in skeletal development resulting in reduced body size, around 3500 BP. The subsequent return to pre-3500 BP skeletal size around 2000 $\mathrm{BP}$ is coincident with the introduction of sheep herding, suggesting that the adoption of this new subsistence strategy was undertaken as a means of alleviating stress. Other studies (e.g. Sadr 2004; Pfeiffer and Sealy 2006; Stynder et al. 2007) have suggested that evidence of stress associated with the intensification of foraging behaviours was experienced earlier (4000 to 3000 BP) by groups inhabiting the Western and Southern Cape region, and that foragers recovered from this stress event prior to the introduction of sheep herding. The nature and magnitude of body size change observed in the Eastern Cape sample corresponds with reductions in stature (Wilson and Lundy 1994, Sealy and Pfeiffer 2000; Pfeiffer and Sealy 2006) and cranial size (Stynder 2006; Stynder et al. 2007a, b) observed among contemporaneous Southern and Western Cape foraging populations. A number of factors, including regional differences in the timing of climatic 
changes, regional variability in species diversity and seasonality, and the nature of the uncalibrated radiocarbon dates may be responsible for this incongruity.

Paleoenvironmental research from across the Cape region suggests that a series of rapid yet major climatic events occurred between 5000 and $2400 \mathrm{BP}$. The timing of these climatic events may have differed somewhat across this region differing with latitude and climate. In the inland regions of the Karoo, an increase in precipitation with corresponding reduction in temperature appears to have occurred around $5000 \mathrm{BP}$ (Lewis 2005). In comparison deposits from the Cango Cave in the Southern Cape suggest that increases in precipitation did not occur until around 3500 BP. Paleoenvironmental research conducted in the Drakensburg region of Eastern Cape reports evidence of a dramatic and short lived wet period from 3200 to $2400 \mathrm{BP}$ (Lewis 2005). The sites sampled suggested an increased importance of browsers in the forager diet supporting an increase in shrubs consequent from increased precipitation and runoff than during previous periods (Lewis 2005:46).

Regional climatic and environmental differences could also have some bearing on the timing of this pattern across the coast. As previously discussed, the range of environmental diversity is variable across the southern coastal region. In the Eastern Cape the Cape Fold Mountains provide less of a barrier to coastal-inland mobility, and the lack of seasonality in rainfall patterns means that floral and faunal resources are more readily available throughout much of the year. It is possible that because of these environmental and climatic differences, that the Eastern Cape populations were somewhat more buffered against the negative effects of the population and subsistence stress. The greater species diversity and year-round availability of resources may have 
allowed Eastern Cape foragers to stave off the effects of this stress longer than groups inhabiting the Western and Southern Capes. This could have delayed the onset of smaller adult size.

It is also possible that this slight temporal discrepancy is an artifact of sample size. Because the current study takes a smaller regional focus, the skeletal sample restricted to the Eastern Cape is smaller than samples use in other skeletal studies studying larger regions. The number of Eastern Cape skeletons dating prior to $4000 \mathrm{BP}$ is quite small in relation to the total sample. It is possible that those individuals with early dates may differentially skew the pattern making the decrease in body size appear more dramatic. The existence of very few pre- $4000 \mathrm{BP}$ specimens is not restricted to this study. It appears to be a symptom of the occupation history of the Cape region. The Later Stone Age skeletal samples of other South African studies that have examined potential skeletal changes through time also suffer from a dearth of individuals that predate 4000 BP (Sealy and Pfeiffer 2001; Pfeiffer and Sealy 2006; Stynder 2006; Stynder et al. 2007). Even though the skeletal samples utilized by these other studies are larger than the Eastern Cape sample, the effect of the distribution of dated skeletons on the results is similar. Nevertheless, when the temporal distribution of individual data points is considered for each anatomical region, the smallest individuals fall within the period of population stress and resource scarcity, suggesting that regardless of sample size, this pattern is not artificial.

Potential radiocarbon calibration issues stemming from the marine reservoir effect may also help to explain the discrepancy in the timing of apparent stress across the Cape region. The radiocarbon dates obtained for the Eastern Cape skeletal sample, like those 
from other LSA human skeletons from the Cape region, are uncalibrated. The lack of calibration is not a major issue if all dated individuals consumed protein from terrestrial sources during their lives. However, antiquity of reported individuals whose diet included a significant contribution from marine protein may appear older than is true. Radiocarbon dates based on bone collagen that were obtained from humans who consumed a diet high in marine foods will appear older than they should be as a result of ocean currents and upwellings that bring old carbon to the surface where marine organisms feed (Ramsey et al 2006). Because Western and Southern Cape forager subsistence was focused on marine resources in particular during the period of intensification, remains associated with that period may yield radiocarbon dates that are artificially older by as much as ca. 300 years. This phenomenon may partly explain the discrepancy in the timing of the skeletal evidence of subsistence stress, with the earlier onset of population stress appearing to occur in the Western and Southern Capes. It has been argued that the marine reservoir effect may not be such an issue for the radiocarbon dates obtained from Eastern Cape LSA remains. Archaeological evidence, namely the absence of large Eastern Cape coastal marine processing sites (Binneman 1995), suggests that Eastern Cape foragers may not have been as reliant on marine resources as other groups living to the west. If this is the case then the temporal documentation of body size reduction and recovery in the Eastern Cape may be more accurate than the observations from other regions as previously documented. Other issues, specifically the presence of CAM plants in the Eastern Cape, will complicate the calibration of radiocarbon dates and therefore affect the timing of the observed patterns of skeletal size change. Nevertheless, regardless of the temporal differences, the presence of a pattern of body size reduction, 
with subsequent recovery, across the southern Cape region, indicates how dramatic and pervasive the effects of subsistence intensification were on foragers across the entire Cape region.

\subsection{Possible explanations for the post-500 BP increase in body size}

Genetic continuity in the southern region of South Africa, specifically the Eastern Cape, throughout the Holocene is supported by the findings of this study. While it has been argued that the pattern and timing of skeletal change in the Eastern Cape is related to population stress and the intensification of subsistence, changes in physique during the period after 2000 BP cannot be attributed to this event. After 2000 BP, body size levels return to those observed prior to the advent of intensification around $3500 \mathrm{BP}$ suggesting that those various stressors had been alleviated. However, the upper and lower limbs continue to increase in size after $2000 \mathrm{BP}$, with the most dramatic increases taking place in the past 500 years. The increases in upper limb length in the last 500 years are most marked for females whereas such a pronounced increase is not present for males. Increases in lower limb length are equal in magnitude for both sexes within the last 500 years compared to the period prior to $4000 \mathrm{BP}$.

Gene flow with the genetically distinct immigrant agriculturalists who entered the region around $1300 \mathrm{BP}$ may serve as a partial explanation for this directional change. South African Bantu-speaking agriculturalists are not only genetically distinct from indigenous LSA groups, but are also larger in body size. The largest individuals in the Eastern Cape sample date from the last 500 years. Size and shape variability is also greatest in the last 500 years. This suggests that gene flow with genetically distinct immigrant agriculturalists may have occurred. 
It is possible that foraging and herding groups may have interacted more extensively with agricultural groups who settled in adjoining areas in the latter part of the late Holocene. Iron Age occupations are restricted to the eastern part of South Africa due to crop requirements of African cereals for summer rainfall (Hall 1987; Huffman 2006). The edge of the summer rainfall zone lies at the Great Fish River, at the eastern edge of the study area. Only two individuals in the study sample have been recovered from burial contexts east of the Great Fish River: ALB 305 from Hamburg (640 \pm 40 BP) and ALB 344 from Gonubie (1957 \pm 26 BP). Other individuals in the Eastern Cape sample with recent radiocarbon dates coinciding with agricultural settlement are located throughout the study area. The proximity of the indigenous foraging ranges to these agricultural settlements will have influenced the frequency and nature of interactions. Hall suggests, citing ethnographic, archaeological and rock art evidence, that the relationship between Eastern Cape foragers and food producers was one of reciprocal exchange and not forced assimilation or displacement (1994). A more direct, albeit potentially biased, picture of intergroup contact comes from the accounts of European missionaries and travelers. Such accounts document San having obtained domestic crops and animals from their neighbours in exchange for marriage partners (Norton 1910), as payment for herding services (Dornan 1907), and in return for spiritual services like rain making (Stanford 1910) (as cited in Loubser and Laurens 1994). Thus, it is possible that individuals who may have practiced a foraging lifestyle, and were buried with forager signatures, possessed a mixed genetic heritage resulting from these interactions. The findings of a craniometric study by A. Morris (1992) in which significant admixture with Bantuspeaking groups was detected in protohistoric Northern Cape pastoralist groups is an 
analogous situation. Even though care was taken to only include individuals with forager and pastoralist archaeological signatures in the current study, gene flow with outside groups is still possible.

\subsection{Chapter Summary and Conclusions}

Skeletal metric and cranial discrete data indicate that substantial cultural continuity was maintained in the Eastern Cape region throughout the Holocene. Some differences in cranial, dental and postcranial metric variables were identified through time, but these are minor and pertain mainly to size. The consistency in cranial discrete trait frequencies between the pre-2000 BP and post-2000 BP subgroups corroborates the metric results and further supports the hypothesis that the introduction of sheep herding was an indigenous invention brought about by diffusion. The multivariate regression results not only provide insights about the continuity of the Eastern Cape inhabitants through the Holocene, but also make it possible to identify a temporal pattern of body size change that correlates with another significant event in southern African prehistory: the intensification of foraging behaviour. The nature and timing of the observed patterns of skeletal change in the Eastern Cape study sample are consistent with a short term period of environmental change and resource scarcity followed by a period of recovery.

The introduction of sheep herding to southernmost Africa has been suggested by some researchers as having adverse effects on indigenous foragers. Others have interpreted increases in stature and body size occurring at around 2000 BP as evidence of the introduction of a distinct population of herders who were taller in stature and larger in body size than foragers (Smith et al. 1992; Sealy and Pfeiffer 2001). The outcomes of the current research on the Eastern Cape Later Stone Age adult skeletal sample suggest 
otherwise. An ultimately transient decrease in cranial, dental and postcranial size between 3500 to $2000 \mathrm{BP}$ is identified. Because the nature of the skeletal change is restricted to size and does not extend to changes in shape, it is concluded that the cause was environmental rather than genetic. The onset of the transient reduction in overall skeletal size does not correspond with the purported introduction of sheep at around 2000 BP. Rather, the decline in body size appears to coincide with the intensification of foraging behaviour, at time when foraging groups were experiencing nutritional insufficiency resulting from the combined negative effects of climate change and increased population density. Furthermore, the scope of the skeletal size modification, extending to areas of the skeleton, namely the cranium and dentition, that are more developmentally constrained and are not as influenced by external pressures as the postcranial region, speaks to the severity of the period of stress. These findings from the LSA foragers of the Eastern Cape are consistent with skeletal size changes identified among contemporaneous groups from the Western and Southern Capes (Pfeiffer and Sealy 2006), and add support to the archaeological evidence of intensification (food storage, increased territoriality, stylistic modifications in lithic manufacture) that has been discovered across the Cape region.

The recovery of body size at around 2000 BP to pre-intensification levels provides important insights regarding the advent of sheep herding in the Cape region of South Africa. The timing and pattern of the body size recovery suggest that some foraging groups may have made the choice to adopt sheep herding as a means to alleviate subsistence stress. This move would not only have provided a stable food source, but would also have partially removed some pressure on the existing local wild food 
resources and encouraged the use of the broader landscape, since suitable grazing land would need to be sought. The apparent return to pre-3500 BP body size levels around 2000 BP suggests that at least some Eastern Cape foragers may have adopted herding to fix their situation. Because the earliest sites in the Cape region with pastoral signatures contain very small quantities of sheep remains it is likely that other factors also contributed to the alleviation of stress that groups were experiencing. The introduction of sheep herding also coincides with a return to more favourable environmental conditions and a redistribution of groups across the landscape which would have also helped to alleviate resource stress. However, other studies that have asserted that foragers were able to recover from the stresses associated with intensification prior to the introduction of sheep (Sealy and Pfeiffer 2006; Stynder et al 2007). Differences in the onset of environmental change between 4000 and 3000 BP and variations in resource availability and seasonality across the Cape region may explain this discrepancy. Radiocarbon calibration issues may also explain this regional variability in the timing of the skeletal evidence of intensification. Future study of the correspondence between calibrated and uncalibrated dates in the Eastern Cape is needed before the findings of the current study can authoritatively call into question existing ideas surrounding the timing of the introduction of sheep herding and the intensification of subsistence behaviours in southernmost Africa.

Congruity in cranial discrete trait frequencies between the two temporally defined subgroups serves as another piece of evidence in support the diffusion hypothesis. The lack of significant differences between these two subgroups indicates that genetic continuity was maintained among groups inhabiting the Eastern Cape throughout the 
Holocene. Similarities in cranial discrete trait frequencies between the entire Eastern Cape sample and other groups with demonstrated genetic affinity reinforces the likelihood that the introduction of herding was the result of a diffusion of ideas whereby sheep were adopted by some indigenous foraging groups while others maintained their traditional foraging lifestyle.

The findings of the current study add new knowledge to our understanding of two important events in the Holocene prehistory of South Africa: the causes and impact of the intensification of foraging behaviour and the mechanisms responsible for the introduction of sheep herding. The present evidence not only brings new information to light about these two episodes of socioeconomic change, but also suggests that causal relationship may have existed. This research makes a significant contribution to the debate surrounding the introduction of pastoralism to southernmost Africa in finding that significant change in skeletal morphology associated with the advent of sheep herding could not be detected at $2000 \mathrm{BP}$, thus supporting the maintenance of population continuity was in the Eastern Cape region throughout the Holocene. The conclusions of this research have relevance for a number of issues in South African prehistory, specifically coastal adaptation, seasonal mobility and the extent of forager mobility and contact. This research also expands on our understanding of forager adaptability and success, especially in an understudied part of the Cape region, the Eastern Cape. 


\section{Literature Cited}

Anonymous. (2007). National Weather [Internet]. South African Weather Service. Available from: http://www/weathersa.co.za

Acocks, J.P.H. (1988). Veld Types of South Africa, $3^{\text {rd }}$ Edition. Memoirs of the Botanical Survey of South Africa No. 57. Department of Agriculture and Water Supply, South Africa.

Ames, K.A. (2004). Supposing hunter-gatherer variability. American Antiquity 69(2): 364374.

Anderson, J.E. (1968). Skeletal "anomalies" as genetic indicators. In The Skeletal Biology of Earlier Human Populations, ed. D.R. Brothwell, pp. 135-147. Oxford: Pergamon Press.

Balasse, M., Ambrose, S.H., Smith, A.B., \& Price, T.D. (2002). The seasonal mobility model for prehistoric herders in the south-western Cape of South Africa assessed by isotopic analysis of sheep tooth enamel. Journal of Archaeological Science 29:917932.

Ball, C.N. \& Connor-Linton, J. (1996). Web Chi Square Calculator. http://www.georgetown.edu/cball/webtools/web_chi.html

Barkhan, D. \& Soodyall, H. (2006). Mitochondrial DNA (mtDNA) and Y chromosome DNA variation in African populations. In The Prehistory of Africa: Tracing the Lineage of Modern Man, ed. H. Soodyall, pp. 139-147. Jeppestown: Jonathan Ball.

Barnes, D.S. (1969). Tooth morphology and other aspects of the Teso Dentition. American Journal of Physical Anthropology 30:183-194.

Beals, K.L., Smith, C.L., \& Dodd, S.M. (1983). Climate and the evolution of brachycephalization. American Journal of Physical Anthropology 62: 425-437.

Bernard, H. Russell. (1995). Research Methods in Anthropology: Qualitative and Quantitative Approaches. Walnut Creek, CA: AltaMira Press.

Berry, A.C. \&Berry, R.J. (1967). Epigenetic variation in the human cranium. Journal of Anatomy 101: 361 - 379. 
Berry, R.J. (1968). The biology of non-metrical variation in mice and men. . In The Skeletal Biology of Earlier Human Populations, ed. D.R. Brothwell, pp. 103-133. Oxford: Pergamon Press.

Bettinger, R.L. (1991). Hunters and Gatherers: Archaeology and Evolutionary Theory. New York: Plenum Press.

Binford, L.R. (2001). Constructing Frames of Reference: An analytical method for archaeological theory building using hunter-gatherer and environmental data sets. Berkeley: University of California Press.

Binneman, J. (1996a). The symbolic construction of communities during the Holocene Later Stone Age in the south-east Cape. PhD Dissertation. University of Witwatersrand. Binneman, J. (1996b). Preliminary report on the investigations at Kulubele, an early Iron Age farming settlement in the Great Kei River valley, Eastern Cape. South African Field Archaeology. 5: 28-35.

Binneman, J. (1997). Results from test excavations at the Heavens Cave, Cambria Valley, Southeast Cape. South African Field Archaeology 6: 93-105.

Binneman, J. (1998). Results from a test excavation at Kleinpoort shelter in the Baviaanskloof, Eastern Cape Province. South African Field Archaeology 7: 90-97.

Binneman, J. (1999). Results from a test excavation at Groot Kommandokloof shelter in the Baviaanskloof/Kouga region, Eastern Cape Province. South African Field Archaeology 8: 100-107.

Binneman, J. (2000). Results from two test excavations in the Baviaanskloof Mountains, Eastern Cape Province. South African Field Archaeology 9: 83-96.

Binneman, J. (2001). An introduction to the Later Stone Age research project along the South-eastern Cape Coast. Southern African Field Archaeology 10:75-87.

Binneman, J. (2004/2005). Archaeological research along the South-Eastern Cape coast part 1: Open-air shell middens. South African Field Archaeology 13 \& 14: 49-77.

Binneman, J. \& Hall, S. (1993). The context of four painted stones from the South-eastern and Eastern Cape. Southern African Field Archaeology 2:89-95.

Blakely, R.L. (1977). Biocultural Adaptation in Prehistoric America. Proceedings No. 11, Southern Anthropological Society. Athens: University of Georgia Press. 
Bogin, B. (1999). Patterns of Human Growth. Cambridge: University Press.

Bogin, B., \& Loucky, J. (1997). Plasticity, political economy, and physical growth status of Guatemala Maya children living in the United States. American Journal of Physical Anthropology 102: 17-32.

Bogin, B., Smith, P., Orden, A.B., Varela Silva, MI, \& Loucky, J. (2002). Rapid change in height and body proportions of Maya American children. American Journal of Human Biology 14: 753-761.

Boonzaier, E., Malherbe, C., Smith, A. \& Berens, P. (1996). The Cape Herders: A History of the Khoikhoi of Southern Africa. Cape Town, SA: David Philip Publishers.

Bousman, C.B. (1998). The chronological evidence for the introduction of domestic stock into southern Africa. African Archaeological Review 15(2): 133-150.

Boyd, D.C.M., \& Boyd, C.C. Jr. (1989). A comparison of Tennessee Archaic and Mississippian maximum femoral lengths and midshaft diameters: Subsistence change and post cranial variability. Southeastern Archaeology 8: 107-116.

Brace, C.L., Smith, S.L. \& Hunt, K.D. (1991). What big teeth you had grandma! Human tooth size past and present. In Advances in Dental Anthropology, eds. M.A. Kelly and C.S. Larsen, pp. 33-58. New York: Wiley-Liss.

Brooks, S.T. \& J.M. Suchey. (1990). Skeletal age determination based on the os pubis: A comparison of the Acsadi-Nemerskeri and Suchey-Brooks methods. Human Evolution 5: 227-238.

Buchanan, W.F., Parkington, J.E., Robey, T.S., \& Vogel, J.C. (1984). Shellfish, subsistence and settlement: Some western Cape Holocene observations. In Frontiers: Southern African Archaeology Today, eds. M. Hall, G. Avery, D.M. Avery, M.L. Wilson, and A.J.B Humphreys, pp. 121-130. BAR International Series 207. Cambridge Monographs in African Archaeology 10.

Buikstra, J.E. \& Beck, L.A. (2006). Bioarchaeology: The Contextual Analysis of Human Remains. Burlington, MA: Academic Press.

Buikstra, J.E. \& Ubelaker, D.H., eds. (1994). Standards for Data Collection from Human Skeletal Remains: Proceedings of a seminar at the Field Museum of Natural History, organized by Jonathan Haas. Arkansas Archaeological Survey Research Series, No. 44. Fayetteville: Arkansas Archaeological Survey. 
Carson, E.A. (2006). Maximum likelihood estimation of human craniometric heritabilities. American Journal of Physical Anthropology 131: 169-180.

Cashdan, E. (1992). Spatial organization and habitat use. In Evolutionary Ecology and Human Behaviour, eds. E.A. Smith and B. Winterhalder, pp. 237-266. Hawthorne, NY: Aldine de Gruyter.

Chen, Y.S., Olckers, A., Schurr, T., Kogelnick, A.M., Houponen, K., \& Wallace, D.C. (2000). MtDNA variation in the South African !Kung and Khwe and their genetic relationship to other African populations. American Journal of Human Genetics 66: 1362-1383.

Churchill, S.E. \& Morris, A.G. (1998). Muscle marking morphology and labour intensity in prehistoric Khoisan foragers. International Journal of Osteoarchaeology 8: 390-411.

Clutton-Brock, J. (1993). The spread of domestic animals in Africa. In The Archaeology of Africa: Food, Metals and Towns, eds. T. Shaw, P. Sinclair, B. Andah, and A. Okpoko, pp. 61-70. New York: Routledge.

Clutton-Brock, J. (1996). The Legacy of Iron Age Dogs and Livestock in Southern Africa. In The Growth of Farming Communities in Africa from the Equator Southwards, ed., J.E.G. Sutton, pp. 161-167. Azania special volume XXIX-XXX, The British Institute in Eastern Africa.

Cohen, M.N. \& Armelagos, G.J. (1984). Paleopathology at the Origins of Agriculture. New York: Academic Press.

Cohen, M.N. (1989). Health and the Rise of Civilization. New Haven, CT: Yale University Press.

Cooper, R.S, Guo, X., Rotimi, C.N., Luke, A., Ward, R., Adeyemo, A., \& Danilov, S.M. (2000). Heritability of angiotensin-converting enzyme and angiotensinogen : a comparison of US blacks and Nigerians. Hypertension 35: 1141-1147.

Cowling, R.M. \& Hilton-Taylor, C. (1997). Phytogeography, flora and endemism. In Vegetation of Southern Africa, eds. R.M. Cowling, D.M. Richardson, and S.M. Pierce, pp. 43-58. Cambridge: University Press.

Cowling, R.M., Richardson, D.M., \& Mustart, P.J. (1997). Fynbos. . In Vegetation of Southern Africa, eds. R.M. Cowling, D.M. Richardson, and S.M. Pierce, pp. 99-123. Cambridge: University Press. 
Crawhall, N. (2006). Languages, genetics and archaeology: problems and possibilities in Africa. In The Prehistory of Africa: Tracing the Lineage of Modern Man, ed. H. Soodyall, pp. 109-122. Jeppestown: Jonathan Ball.

Cunningham, A.B. \& Davis, G.W. (1997). Human use of plants. In Vegetation of Southern Africa, eds. R.M. Cowling, D.M. Richardson, and S.M. Pierce, pp. 474-501. Cambridge: University Press.

Cybulski, J.S. (1992). A Greenville Burial Ground: Human Remains and Mortuary Elements in British Columbia Coast Prehistory. Archaeological Survey of Canada, Mercury Series Paper No. 146. Ottawa: Canadian Museum of Civilization

Damuth, J. (1981). Population density and body size in mammals. Nature 290: 699-700.

Deacon, H.J. (1976). Where Hunters Gathered: A study of Holocene Stone Age people in the Eastern Cape. South African Archaeological Society Monograph Series, No 1. Claremont, SA.

Deacon, HJ. (1993). Planting an idea: An archaeology of Stone Age gatherers in South Africa. South African Archaeological bulletin 48: 86-93.

Deacon, HJ. (1992). Human Settlement. In The Ecology of Fynbos: Nutrients, Fire and Diversity, ed. R.M. Cowling, pp. 260-270. Cape Town: Oxford University Press.

Deacon, J. (1984). Later Stone Age people and their descendants in southern Africa. In Southern African Prehistory and Paleoenvironments, ed. R.G. Klein, pp. 221-328. Rotterdam, ND: A.A. Balkema Publishers.

Deacon, H.J. \& Deacon, J. (1999). Human Beginnings in South Africa: Uncovering the Secrets of the Stone Age. Walnut Creek, CA: AltaMira Press.

DeLaurier, A. \& M.W. Spence. (2003). Cranial genetic markers: Implications for postmartial residence patterns. In Bones of the Ancestors: The Archaeology and Osteobiography of the Moatfield Site, eds. R.F. Williamson and S. Pfeiffer, pp. 263294. Archaeological Survey of Canada Mercury Series Paper 163. Ottawa: Canadian Museum of Civilization.

Denbow, J.R. (1999). Material culture and the dialects of identity in the Kalahari: AD 7001700. In Beyond chiefdoms: pathways to complexity in Africa, ed. S.K. McIntosh, pp. 110-123. Cambridge: Cambridge University Press. 
Denbow, J.R, \& Wilmsen, E.N. (1986). Advent and course of pastoralism in the Kalahari. Science 234: 1509-1515.

De Villiers H. (1968). The skull of the South African Negro: A biometrical and morphological study. Johannesburg: Witwatersrand University Press.

Dewar, G.I. (2007). The archaeology of the coastal desert of Namaqualand, South Africa: a regional synthesis. PhD Dissertation. University of Cape Town, South Africa.

Dewar, G. \& Pfeiffer S. (2004). Postural behaviour of Later Stone Age people in South Africa. South African Archaeological Bulletin 59:52-58.

Dobres, M-A, \& Robb, J.E. (2000). Agency in Archaeology. London: Routledge.

Donlon, D.A. (2000). The value of infracranial nonmetric variation in studies of modern Homo sapiens: An Australian focus. American Journal of Physical Anthropology 113: 349-368.

Drennan, M.R. (1929). The dentition of a Bushman tribe. Annals of the South African Museum 24: 61-87.

Durham, W.H. (1981). Overview: Optimal foraging analysis in human ecology. In HunterGatherer Foraging Strategies: Ethnographic and Archaeological Analyses, eds. B. Winterhalder and E.A. Smith, pp. 218-231. Chicago: University of Chicago Press.

Eerkins, J.W. \& Lipo, C.P. (2007). Cultural transmission theory and the archaeological record: Providing context to understanding variation and temporal changes in material culture. Journal of Archaeological Research 15: 239-274.

Elphick, R. (1977). Kraal and Castle: Khoikhoi and the Founding of White South Africa. New Haven: Yale University Press.

Feldesman, M.R., Kleckner, J.G., \& Lundy, J. K. (1991). Femur/stature ratio and estimates of stature in mid- and late-Pleistocene fossil hominids. In Paleoanthropology Annuals, vol. 1, eds. E. Delson, I. Tattersal, and J. van Couvering, pp. 139-150. New York: Garland.

Finlayson, R. (2006). Linguistic relationships: how genetic are they? . In The Prehistory of Africa: Tracing the Lineage of Modern Man, ed. H. Soodyall, pp. 125-138. Johannesburg: Jonathan Ball Publishers. 
Franciscus, R.G., \& Long, J.C. (1991). Variation in human nasal height and breadth. American Journal of Physical Anthropology 85: 419-427.

Gabriel, S.B. et al. (2002). The structure of haplotype blocks in the human genome. Science 296: $2225-2229$.

Gifford-Gonzalez, D. (1998). Early pastoralists in East Africa: Ecological and social dimensions. Journal of Anthropological Archaeology 17: 166-200.

Gifford-Gonzalez, D. (2000). Animal disease challenges to the emergence of pastoralism in Sub-Saharan Africa. African Archaeological Review 17(3): 95-139.

Ginter, J.K. (2005). Dental health of LSA inhabitants of the Eastern Cape, South Africa. Gaborone, Botswana: Unpublished paper presented at the $12^{\text {th }}$ Congress of the Panafrican Archaeological Association for Prehistory and Related Studies, July 3-10. Garn, S.M, Lewis, A.B. \& Walenga, A. (1968). Evidence for a secular trend in tooth size over two generations. Journal of Dental Research 47(3): 503.

Gilligan, I., \& Bulbeck, D. (2007). Environment and morphology in Australian Aborigines: A re-analysis of the Birdsell database. American Journal of Physical Anthropology 134: 75-91.

Guglielmino-Matessi, C.R., Gluckman, P., \& Cavalli-Sforza, L.L. (1979). Climate and the evolution of skull metrics in man. American Journal of Physical Anthropology 50: $549-564$.

Hall, M. (1987). The Changing Past: Farmers, Kings and Traders in southern Africa. Cape Town: David Philip.

Hall, S.L. (1986). Pastoral adaptations and forager relations in the eastern Cape. Prehistoric Pastoralism in Southern Africa. South African Archaeological Society, Goodwin Series 5:42-49.

Hall, S. (1990). Hunter-fisher-gatherers of the Fish River Basin: A contribution to the Holocene prehistory of the Eastern Cape. PhD Dissertation. University of Stellenbosch.

Hall, S. (2000). Burial and sequence in the Later Stone Age of he Eastern Cape Province, South Africa. South African Archaeological Bulletin 55: 137-146. 
Hall, S. \& Binneman, J. (1987). Later Stone Age burial variability in the Cape: A social interpretation. South African Archaeological Bulletin 42: 140-152.

Hanihara, T., Ishida, H., \& Dodo, Y. (2003). Characterization of biological diversity through analysis of discrete cranial traits. America Journal of Physical Anthropology 121: $241-251$.

Hansen, J.D.L., Dunn, D.S., Lee, R.B., Becker, P.J., \& Jenkins, T. (1993). Hunter-gatherer to pastoral way of life: effects of the transition on health, growth and nutritional status. South African Journal of Science 89: 559-564.

Harpending, H.C., \& Ward, R. (1982). Chemical systematics and human evolution. In Biochemical Aspects of Evolutionary Biology, ed. M. Nitecki, pp. 213-256. Chicago: University of Chicago Press.

Harris, E.F., Potter, R.H, \& Lin, J. (2001). Secular trend in tooth size in urban Chinese assessed from two-generation family data. American Journal of Physical Anthropology 115: 312-318.

Hauser, G., \& De Stefano, G.F. (1989). Epigenetic Variants of the Human Skull. Stuttgart: Schweizerbart.

Hausman, A.J. (1982a). Holocene human evolution in southern Africa: the biocultural development of the Khoisan. PhD Dissertation. State University of New York, Binghamton, USA.

Hausman A.J. (1982b). The biocultural evolution of Khoisan populations of Southern Africa. American Journal of Physical Anthropology 58: 315-330.

Hazard Munro, B. (1997). Statistical Methods for Health Care Research. Philadelphia: Lippincott-Raven Publishers.

Henshilwood, C. (1996). A revised chronology for pastoralism in southernmost Africa: New evidence for sheep at ca. 2,000 BP from Blombos Cave, South Africa. Antiquity 70: 945-949.

Henshilwood, C.S. \& Marean, C. W. (2006). Remodeling the origins of modern human behaviour. In The Prehistory of Africa: Tracing the Lineage of Modern Man, ed. H. Soodyall, pp. 33-46. Jeppestown: Jonathan Ball. 
Henshilwood, C.S., Nilssen, P., \& Parkington, J. (1994). Mussel drying and food storage in the Late Holocene, SW Cape, South Africa. Journal of Field Archaeology 21(1): 103-109.

Hillson, S., C. FitzGerald, \& Flinn, H. (2005). Alternative dental measurements: Proposals and relationships with other measurements. American Journal of Physical Anthropology 126: 413-426.

Hoffman, M.T. (1997). Human impacts on vegetation. . In Vegetation of Southern Africa, eds. R.M. Cowling, D.M. Richardson, and S.M. Pierce, pp.507-531. Cambridge: University Press.

Holl, A.F.C. (1998). Livestock husbandry, pastoralisms, and territoriality: The West African record. Journal of Anthropological Archaeology 17: 143-165.

Holliday, T.W. (1995). Body size and proportions in the Late Pleistocene Western Old World and the origins of modern humans. PhD Dissertation. Department of Anthropology, University of New Mexico.

Howells, W.W. (1995). Who's who in skulls: ethnic identification of crania from measurements. Cambridge, MA: Harvard University Press.

Huffman, T.N. (1996). Archaeological evidence for climatic change during the last 2000 years in southern Africa. Quaternary International 33: 55-60.

Huffman, T.N. (2006). Bantu migrations in southern Africa. In The Prehistory of Africa: Tracing the Lineage of Modern Man, ed. H. Soodyall, pp. 97-108. Jeppestown: Jonathan Ball.

Ingman, M., Kaessmann, H., Paabo, S., \& Gyllensten, U. (2000). Mitochondrial genome variation and the origin of modern humans. Nature 408: 708-713.

Inskeep, R. (1986). A preliminary survey of burial practices in the Later Stone Age, from the Orange River to the Cape coast. In Variation, Culture, and Evolution in African Populations: Papers in Honour of Dr. Hertha de Villiers, eds. R. Singer and J.K. Lundy, pp. 212-219. Johannesburg: Witwatersrand University Press.

Iscan, M.Y., S.R. Loth, \& R K. Wright. (1984). Age estimation from the rib by phase analysis: White males. Journal of Forensic Science 29(4):1094-1104.

Iscan, M.Y., S.R. Loth, \& R K. Wright. (1985). Age estimation from the rib by phase analysis: White females. Journal of Forensic Sciences 30(3):853-863. 
Jacobson, A. (1982). The Dentition of the South African Negro. Anniston, AL: Higginsbotham.

Jerardino Wiesenborn, A.M.S. (1996). Changing social landscapes of the Western Cape coast of South Africa over the last 4500 years. PhD Dissertation. University of Cape Town.

Jerardino, A. (1997). Changes in shellfish species composition and mean shell size from a Late-Holocene record of the west coast of southern Africa. Journal of Archaeological Science 24: 1031-1044.

Jerardino, A., Branch, G.M, \& Navarro, R. (2008). Human impact on precolonial West Coast marine environments of South Africa. In Human Impacts of Marine Environments: A Global Perspective, eds. J.M. Erlandson \& T.C. Rick, pp. 279-296. Berkeley: University of California Press.

Jerardino, A., Sealy, J., \& Pfeiffer, S. (2000). An infant burial from Steenbokfontein Cave, West Coast, South Africa: Its archaeological, nutritional, and anatomical context. South African Archaeological Bulletin 55: 44-48.

Jenkins, T., Zoutendyk, A., \& Steinberg, A.G. (1970). Gammaglobulin groups (Gm and Inv) of various Southern African populations. American Journal of Physical Anthropology 32: 197-218.

Johnston, F. E. (1968). Growth of the Skeleton in Earlier Peoples. In The Skeletal Biology of Earlier Human Populations, ed. D.R. Brothwell, pp. 57-66. Oxford: Pergamon Press

Jolicoeur, P. \& Mosimann, J.E. (1960). Size and shape variation in the painted turtle. A principal component analysis. Growth 24: 339-354.

Jorde, L.B., Watkins, W.S., Bamshad, M.J., Dixon, M.E., Ricker, C.E., Seielstad, M.T., \& Batzer, M.A. (2000). The distribution of human genetic diversity: a comparison of mitochondrial, autosomal, and Y-chromosome data. American Journal of Human Genetics 66: 979-988.

Kaplan, H. \& Hill, K. (1992). The evolutionary ecology of food acquisition. In Evolutionary Ecology and Human Behaviour, eds. E.A. Smith and B. Winterhalder, pp. 168-201. Chicago: University of Chicago Press. 
Katzmarzyk, P.T., \& Leonard, W.R. (1998). Climatic influences on human body size and proportions: Ecological adaptations and secular trends. American Journal of Physical Anthropology 106: 483-503.

Keiser, J.A. (1985). An odontometric analysis of the early Griqua dentition.

Anthropolgischer Anzeiger 43: 51-58.

Keiser, J.A., Groeneveld, H.T., \& Cameron, N. (1987). Evidence for a secular trend in the Negro dentition. Annals of Human Biology 14: 517-532.

Kelly, R.L. (1995). The foraging spectrum: diversity in hunter-gatherer lifeways.

Washington, D.C.: Smithsonian Institution Press.

Kennedy, G.E. (1986). The relationship between auditory exostoses and cold water: a latitudinal analysis. American Journal of Physical Anthropology 71: 401-415.

Klein, R.G. (1994). Southern Africa before the Iron Age. In Integrative Paths to the Past: Paleoanthropological Advances in Honour of F. Clark Howell, eds. R.S. Corruccini and R.L. Ciochon, pp. 471-519. Englewood Cliffs, NJ: Prentice Hall.

Kinahan, J. (2001). Pastoral Nomads of the Namib Desert: The people history forgot. Windhoek, Namibia: Capital Press.

Kurki, H.K., Ginter, J.K, Stock, J.T., \& Pfeiffer, S. (2007). Body size reconstruction in a small-bodied sample: applicability of current methods. Philadelphia, PA:

Unpublished paper presented at the $76^{\text {th }}$ annual meeting of the American Association of Physical Anthropologists, March 28-31.

Kusimba, S.B. (2002). African foragers: environment, technology, interactions. Walnut Creek, CA: AltaMira Press.

Lahr, M.M. (1996). The evolution of modern human diversity: A study of cranial variation. Cambridge: University Press.

Lahr, M.M \& Foley, R.A. (1998). Towards a theory of modern human origins: geography, demography and diversity on recent human evolution. Yearbook of Physical Anthropology 41:137-176.

Larsen, C.S. (1997). Bioarchaeology: Interpreting behaviour from the human skeleton. Cambridge: University Press.

Larsen, C.S. (2002). Bioarchaeology: The lives and lifestyles of past people. Journal of Archaeological Research 10(2): 119-166. 
Lee, R.B. (1968). What hunters do for a living, or how to make out on scarce resources. In Man the Hunter, eds. R.B. Lee and I. Devore, pp. 30-43. Chicago, IL: Aldine Publishing Company.

Lee, R.B. (1993). The Dobe Ju/'hoansi, $2^{\text {nd }}$ Edition. Fort Worth, TX: Harcourt Brace Publishers.

Lee, R.B., and Devore, I., eds. (1968). Man the Hunter. Chicago, IL: Aldine Press.

Lee-Thorp, J.A., Holmgren, K., Lauritzen, S.-E., Linge, H., Moberg, A., Partridge, T.C., Stevenson, C., \& Tyson, P.D. (2001). Rapid climate shifts in the southern African interior throughout the mid to late Holocene. Geophysical Research Letters, 28(23): 4507-4510.

Lewis, C.A. (2002). Radiocarbon dates and the Late Quaternary palaeogeography of the Province of the Eastern Cape, South Africa. Quaternary International 89: 59-69.

Lewis, C.A. (2005). Late Glacial and Holocene palaeoclimatology of the Drakensberg of the Eastern Cape, South Africa. Quaternary International 129: 33-48.

Lewis, C.A. \& Illgner, P.M. (2001). Late Quaternary glaciation in southern Africa: moraine ridges and glacial deposits at Mount Enterprise in the Drakensberg of the Eastern Cape Province, South Africa. Journal of Quaternary Science 16(4): 365-374.

Loubser, J.N.H., \& Laurens, G. (1994). Depictions of domestic ungulates and shields: huntergatherers and agro-pastoralists in the Caledon River Valley area. In Contested Images: Diversity in southern African rock art research, eds. T.A. Dowson and D. Lewis-Williams, pp.83-118. Johannesburg: Witwatersrand University Press.

Louw, J.A., \& Finlayson, R. (1990). Southern Bantu origins as represented by Xhosa and Tswana. Southern African Journal of African Languages 10(4): 401-410.

Lovejoy, C.O., R.S. Meindl, T.R. Pryzbeck, \& R.P. Mensforth. (1985). Chronological metamorphosis of the auricular surface of the ilium: A new method for the determination of adult skeletal age at death. American Journal of Physical Anthropology 68:15-28.

Lubke, R.A., Avis, A.M, Steinke, T.D, \& Boucher, C. (1997). Coastal Vegetation. In Vegetation of Southern Africa, eds. R.M. Cowling, D.M. Richardson, and S.M. Pierce, pp.300-319. Cambridge: University Press. 
Lundy, J.K. (1986). Intertribal metrical and morphological variation in the postcranial skeleton of the South African Negro. In Variation, Culture, and Evolution in African Populations, eds. R. Singer and J.K. Lundy, pp.85-99. Johannesburg: Witwatersrand University Press.

Madrigal, L. (1995). Statistics for Anthropology. Cambridge: University Press.

Maggs, T. (1996). The Early Iron Age in the extreme south: some patterns and problems. In The Growth of Farming Communities in Africa from the Equator Southwards, ed. J.E.G. Sutton, pp. 171-178. Azania special volume XXIX-XXX, The British Institute in Eastern Africa.

Manhire, A.H., Parkington, J.E., Mazel, A.D. \& Maggs, T.M.O'C. (1986). Cattle, sheep, and horses: a review of domestic animals in the rock art of southern Africa. Prehistoric Pastoralism in Southern Africa. South African Archaeological Society, Goodwin Series 5: 22-30.

Mann R.W., R.L. Jantz, W.M. Bass, and P.S. Willey. (1991). Maxillary suture obliteration: A visual method for estimating skeletal age. Journal of Forensic Science 36: 781791.

Marean, C.W. (1992). Hunter to herder: Large mammal remains from the hunter-gatherer occupation at Enkapune Ya Moto rock-shelter, Central Rift, Kenya. African Archaeological Review 10:65-127.

Marshall, F. (1990). Origins of Specialized Pastoral Production in East Africa. American Anthropologist 92(4): 873-894.

Marshall, F. (2000). The origins and spread of domestic animals in East Africa. In The Origins and Development of African Livestock: Archaeology, Genetics, Linguistics and Ethnography, eds. R.M. Blench and K.C. MacDonald, pp. 191-221.

Marshall, F. \& Hildebrand, E. (2002). Cattle before crops: The beginnings of food production in Africa. Journal of World Prehistory 16(2): 99-143.

Mayewski, P.A., Rohling, E.E., Stager, J.C., Wibjorn, K., Maasch, K.A., Meeker, L.D., Meyerson, E.A., Gasse, F., van Kreveld, S., Holmgren, K., Lee-Thorp, J., Rosqvist, G., Rack, F., Staubwasser, M., Schneider, R.R., \& Steig, E.J. (2004). Holocene climate variability. Quaternary Research 62: 243-255. 
Mazel, A.D. (1989). People making history: the last ten thousand years of hunter-gatherer communities in the Thukela Basin. Natal Museum Journal of Humanities 1:1-168.

Meindl, R. S. \& C.O. Lovejoy. (1985). Ectocranial suture closure: A revised method for the determination of skeletal age at death based on the lateral-anterior sutures. American Journal of Physical Anthropology 68:57-66.

Midgely, J.J., Cowling, R.M, Seydack, A.H.W., \& van Wyk, G.F. (1997). Forest. In Vegetation of Southern Africa, eds. R.M. Cowling, D.M. Richardson, and S.M. Pierce, pp. 278-296. Cambridge: University Press.

Mitchell, P. (2002a). Hunter-gatherer archaeology in southern Africa: Recent research, future trends. Before Farming 1(3): 1-36.

Mitchell, P. (2002b). The Archaeology of Southern Africa. Cambridge: University Press. Molto, J.E. (1983). Biological Relationships of Southern Ontario Woodland Peoples: The Evidence of Discontinuous Cranial Traits. National Museum of Man Mercury Series, Archaeological Survey of Canada, Paper 117. Ottawa: National Museum of Man.

Morris, A.G. (1986). Khoi and San Craniology: A re-evaluation of the osteological reference samples. In Variation, Culture and Evolution in African Populations: Papers in Honour of Dr. Hertha de Villiers, eds. R. Singer and J.K. Lundy, pp.1-12. Johannesburg: Witwatersrand University Press.

Morris, A.G. (1987). The reflection of the collector: Khoi and San skeletons in museum collections. South African Archaeological Bulletin 42(145): 12-22.

Morris, A.G. (1992a). A master catalogue: Holocene human skeletons from South Africa. Johannesburg: Witwatersrand University Press.

Morris, A.G. (1992b). The Skeletons of Contact: A Study of Protohistoric Burials from the Lower Orange River Valley, South Africa. Johannesburg: Witwatersrand University Press.

Morris, A.G. (1992c). Biological relationships between the Upper Pleistocene and Holocene populations in southern Africa. In Continuity or Replacement: Controversies in Homo sapiens evolution, eds. G. Grauer and F. Smith, pp. 131-143. Rotterdam: A.A. Balkema. 
Morris, A.G. (1993). Human remains from the Early Iron Age sites of Nanda and KwaGandaganda, Mngeni Valley, Natal, South Africa. Natal Museum Journal of Humanities 5: 83-98.

Morris, A.G. (2002). Isolation and the origin of the Khoisan: Late Pleistocene and Early Holocene human evolution at the southern end of Africa. Human Evolution 7: 231240.

Morris, A.G. (2003). The myth of the East African "Bushmen". South African Archaeological Bulletin 58: 85-90.

Morris, A.G. \& Ribot, I. (2006). Morphometric cranial identity of prehistoric Malawians in the light of Sub-Saharan African diversity. American Journal of Physical Anthropology 130:10-25.

Murcoch, W.W. (1994). Population regulation in theory and practice. Ecology 75: 271-287.

Netting, R.M. (1986). Cultural Ecology, $2^{\text {nd }}$ Edition. Prospect Heights, IL: Waveland Press.

Nurse, G.T., Weinter, J.S., \& Jenkins T. (1985). The Peoples of Southern Africa and their Affinities. Oxford: Clarendon Press.

O'Connor, T.G. \& Bredenkamp, G. J. (1997). Grassland. In Vegetation of Southern Africa, eds. R.M. Cowling, D.M. Richardson, and S.M. Pierce, pp.215-245. Cambridge: University Press.

Ousley, S.D., Billeck, W.T., \& Hollinger, R.E.. (2005). Federal repatriation legislation and the role of physical anthropology in repatriation. Yearbook of Physical Anthropology 48: 2-32.

Parkington, J.E. (1972). Seasonal mobility in the Later Stone Age. African Studies 31: 223243.

Parkington, J.E. (2001). Mobility, seasonality and southern African hunter-gatherers. South African Archaeological Bulletin 56 (173 \& 174):1-7.

Parsons, I. (2006). Later Stone Age socio-economic variability in the Northern Cape, South Africa. Calgary, AB: Unpublished paper presented at the $18^{\text {th }}$ biennial conference of the Society of Africanist Archaeologists, June 23-26.

Pearson, O.M. (2000). Activity, climate and postcranial robusticity: Implications for modern human origins and scenarios of adaptive change. Current Anthropology 41(4): 569-589. 
Pfeiffer, S. (2001). Patterns of skeletal trauma during the Holocene Later Stone Age, Southern Africa. Paper presented at the Pan-African Congress of Prehistory, Bamako, Mali, February 2001.

Pfeiffer, S. (2007). The health of foragers: people of the Later Stone Age, southern Africa. In Ancient Health: Skeletal Indicators of Agricultural and Economic Intensification, eds. M.N Cohen and G. Crane-Kramer, pp. 223-236. Gainesville: University of Florida Press.

Pfeiffer, S. (in press). Cranial trauma as evidence of a stressful period among southern African foragers. In Volume in Honour of Michael Spence eds. P. Timmins and N. Ferris. London, ON: Ontario Archaeological Society.

Pfeiffer, S. \& Crowder, C. (2004). An Ill Child among Mid-Holocene Foragers of Southern Africa. American Journal of Physical Anthropology 123: 23-29.

Pfeiffer, S. \& Sealy, J. (2006). Body Size Among Holocene Foragers of the Cape Ecozone, Southern Africa. American Journal of Physical Anthropology 129: 1-11.

Pfeiffer, S. \& Stock, J. (2002). Upper limb morphology and the division of labour among southern African Holocene foragers. American Journal of Physical Anthropology S34: 124.

Pfeiffer, S., \& van der Merwe, N.J. (2004). Cranial injuries to Later Stone Age children from the Modder River Mouth, Southwestern Cape, South Africa. South African Archaeological Bulletin 59:59-65.

Pfeiffer, S., van der Merwe, N.J., Parkington, J.E., \& Yates, R. (1999). Violent human death in the past: a case from the Western Cape. South African Journal of Science 95: 137140.

Phenice, T.W. (1969). A newly developed visual method of sexing the os pubis. American Journal of Physical Anthropology 30:297-302.

Pimentel, R.A. (1992). An introduction to ordination, principal components analysis and discriminant analysis. In Ordination in the Study of Morphology, Evolution and Systematics of Insects: Applications and Quantitative Genetic Rationals, eds. J.T. Sorensen and R. Foottit, pp. 11-28. Amsterdam: Elsevier Press. 
Powell, J.F., \& Neves, W.A. (1999). Craniofacial morphology of the first Americans: Pattern and process in the peopling of the New World. Yearbook of Physical Anthropology 42:153-188.

Powell, M.L., Bridges, P.S., \& Wagner Mires, A. M. (1991). What Mean these Bones? Studies in Southeastern Bioarchaeology. Tascaloosa: University of Alabama Press.

Price, T.D. \& Brown, J.A. (1985). Aspects of hunter-gatherer complexity. In Prehistoric Hunter-Gatherers: The emergence of cultural complexity, eds. T.D. Price and J.A. Brown, pp.3-20. Orlando: Academic Press.

Prins, F.E. (1996). Climate, Vegetation and Early Agriculturalist Communities in Transkei and KwaZulu-Natal. In The Growth of Farming Communities in Africa from the Equator Southwards, ed. J.E.G. Sutton, pp. 179-186. Azania special volume XXIXXXX, The British Institute in Eastern Africa.

Prowse, T.L, \& Lovell, N.C. (1996). Concordance of cranial and dental morphological traits and evidence for endogamy in ancient Egypt. American Journal of Physical Anthropology 101: 2437-246.

Ramsey, C.B., Buck, C.E., Manning, S.W. Reimer, P. \& van der Pilcht, H. (2006). Developments in radiocarbon calibration for archaeology. Antiquity 80: 783-798.

Relethford, J.H. (2004). Boas and beyond: Migration and craniometric variation. American Journal of Human Biology 16: 379-386.

Relethford, J.H., \& Blangero, J. (1990). Detection of differential gene flow from patterns of quantitative variation. Human Biology 62(1): 5-25.

Relethford, J.H, Crawford, MH, \& Blangero, J. (1997). Genetic drift and gene-flow in post famine Ireland. Human Biology 69: 443-465.

Ribot, I. (2002). Craniomandibular variation in sub-Saharan Africa: sexual dimorphism, geography, ecology and history. PhD Dissertation. Cambridge, University of Cambridge.

Ribot, I. (2003). Craniometric analysis of Central and East Africans in relation to history. Anthropologica et Praehistorica 114:25-50.

Ribot, I. (2004). Differentiation of modern Sub-Saharan African populations: Craniometric interpretations in relation to geography and history. Bulletins et Memoires de la Societe d'Antrhopologie de Paris 16:143-65. 
Rightmire G.P. (1970) Bushman, Hottentot and South African Negro crania studied by distance and discrimination. American Journal of Physical Anthropology 33: 169196.

Rightmire, G.P. (1972). Cranial measurements and discrete traits compared in distance studies of African Negro skulls. Human Biology 44: 263-276.

Rightmire, G.P. (1976). Metric versus discrete traits in African skulls. In The Measures of Man: Methodologies in Biological Anthropology, eds. E. Giles and J.S. Freidlaender, pp.383-407. Cambridge: Peabody Museum Press.

Robbins, L.H., Murphy, M.L., Stewart, K.M, Campbell, A.C., \& Brook, G.A. (1994). Barbed bone points, paleoenvironment, and the antiquity of fish exploitation in the Kalahari Desert, Botswana. Journal of Field Archaeology 21(1): 257-264.

Robbins, L.H., Murphy, M.L., Brook, G.A., Ivester, A.H., Campbell, A.C., Klein, R.G., Milo, R.G., Stewart, K.M., Downey, W.S., \& Stevens, N.J. (2000). Archaeology, palaeoenvironment, and chronology of the Tsodilo Hills White Paintings rock shelter, northwest Kalahari Desert, Botswana. Journal of Archaeological Science 27: 10851113.

Roseman, C., \& Weaver, T.D. (2004). Multivariate apportionment of global human craniometric diversity. American Journal of Physical Anthropology 125: 257-263.

Rosen, D.Z, Lewis, C.A., \& Illgner, P.M. (1999). Paleoclimatic and archaeological implications of organic rich sediments at Tiffindel Ski Resort, near Rhodes, Eastern Cape, South Africa. Transmissions of the Royal Society of South Africa 54(2): 311321.

Rubini, M., Mogliazza, S., \& Corruccini, R.S.T. (2006). Biological divergence and equality during the first millennium $\mathrm{BC}$ in human populations of central Italy. American Journal of Human Biology 19: 119-131.

Ruff, C.B. (1991). Climate, body size, and body shape in hominid evolution. Journal of Human Evolution 21: 81-105.

Ruff, C.B. (2000). Biomechanical analyses of archaeological human skeletons. In Biological Anthropology of the Human Skeleton, eds. M.A. Katzenberg and S.R. Saunders, pp. 71-102. New York: Wiley-Liss. 
Rutherford, M.C. (1997). Categorization of biomes. In Vegetation of Southern Africa, eds. R.M. Cowling, D.M. Richardson, and S.M. Pierce, pp.91-97. Cambridge: University Press.

Sadr, K. (1998). The first herders at the Cape of Good Hope. African Archaeological Review 15(2): 101-132.

Sadr, K. (2003). The Neolithic of Southern Africa. Journal of African History 44: 195-209.

Sadr, K. (2004). Feasting on Kasteelberg? Early herders on the west coast of South Africa. Before Farming: The Archaeology of Old World Hunter-Gatherers 2004/3 (2). http://waspjournals.com.

Sadr, K. (2005). Hunter-gatherers and herders of the Kalahari during the Late Holocene. In. Desert Peoples: Archaeological Perspectives, eds. P. Veth, M. Smith, and P. Hiscock, pp. 206-221. Malden, MA: Blackwell Publishing.

Sadr, K. (2006). Through thick and thin: on early ceramics in southern Africa. Calgary, AB: Unpublished paper presented at the $18^{\text {th }}$ biennial conference of the Society of Africanist Archaeologists, June 23-26.

Sadr, K., Smith, A., Plug, I., Orton, J., \& Mutti, B. (2003). Herders and foragers on Kasteelberg: Interim report of excavations 1999-2002. South African Archaeological Bulletin 58: 27-32.

Schapera, I. (1930). The Khoisan Peoples of South Africa: Bushmen and Hottentots. London: Routledge \& Kegan Paul Ltd.

Scherer, A.K. (2007). Population structure of the Classic Period Maya. American Journal of Physical Anthropology 132:367-380.

Schillaci, M.A., \& Stojanowski, C.M. (2005). Craniometric variation and population History of the Prehistoric Tewa. American Journal of Physical Anthropology 126: 401-412.

Schmid, P.E., Tokeshi, M., \& Schmid-Araya, J.M. (2000). Relation between population density and body size in stream communities. Science 289: 1557-1560.

Schulze, R.E. (1997a). Climate. In Vegetation of Southern Africa, eds. R.M. Cowling, D.M. Richardson, and S.M. Pierce, pp. 21-41. Cambridge: University Press.

Schulze, R.E. (1997b). South African Atlas of Agrohydrology and Climatology. Pietermartizburg, S.A.: Department of Agricultural Engineering, University of Natal. 
Schweitzer, F.R. (1974). Archaeological evidence for sheep at the Cape. South African Archaeological Bulletin 29:75-82.

Sciulli, P.W. (1997). Dental evolution in prehistoric Native Americans of the Ohio Valley Area. I. Wear and pathology. International Journal of Osteoarchaeology 7:507-524.

Sealy, J. (1986). Stable Carbon Isotopes and Prehistoric Diets I the South-Western Cape Province, South Africa. Cambridge Monographs in African Archaeology 15. BAR International Series 293.

Sealy, J. (2002). Letter to the Editor. South African Archaeological Bulletin 57(175): 57.

Sealy, J.C. (2006). Diet, mobility, and settlement patterns among Holocene hunter-gatherers in southernmost Africa. Current Anthropology 47(4): 569-595.

Sealy, J.C., Patrick, M.K., Morris, A.G., \& Alder, D. (1992). Diet and dental caries among Later Stone Age inhabitants of the Cape Province, South Africa. American Journal of Physical Anthropology 88:123-134.

Sealy, J.C. \& Pfeiffer, S. (2000). Diet, body size and landscape use among Holocene people in the Southern Cape, South Africa. Current Anthropology 41(4):642-655.

Sealy, J., Pfeiffer, S., Yates, R., Willmore, K., Manhire, A., Maggs, T., \& Lanham J. (2000). Hunter-gatherer child burials from the Parkhuis Mountains, Western Cape: Growth, diet and burial practices in the Late Holocene. South African Archaeological Bulletin 55: $32-43$.

Sealy, J.C., \& van de Merwe, N.J. (1988). Social, spatial and chronological patterning in marine food use as determined by $\delta^{13} \mathrm{C}$ measurements of Holocene human skeletons from the south-western Cape, South Africa. World Archaeology 20(1): 87-102.

Sealy, J.C. \& van de Merwe, N.J. (1986). Isotope assessment and the seasonal mobility hypothesis in the Southwestern Cape of South Africa. Current Anthropology 27(2): $135-150$.

Sealy, J.C. \& Yates, R. (1994). The chronology of the introduction of pastoralism to the Cape, South Africa. Antiquity 68:58-67.

Shennan, S.J. (2001). Demography and cultural innovation: A model and its implication for the emergence of modern human culture. Cambridge Archaeological Journal 11:516. 
Silberbauer, F.B. (1979). Stable Carbon isotopes and prehistoric diets in the Eastern Cape Province, South Africa. MA thesis. Department of Archaeology, University of Cape Town.

Sinclair, D., \& Dangerfield, P. (1998). Human Growth After Birth, Sixth Edition. Oxford: Oxford University Press.

Smith, A.B. (1994). Metaphors of space: Rock art and territoriality in southern Africa. In Contested Images: Diversity in southern African rock art research, eds. T.A. Dowson and D. Lewis-Williams, pp.373-384. Johannesburg: Witwatersrand University Press.

Smith, A.B. (1986). Competition, conflict, and clientship: Khoi and San relationships in the Western Cape. Prehistoric Pastoralism in Southern Africa. South African Archaeological Society, Goodwin Series 5: 36-41.

Smith, A.B. (1998). Keeping people on the periphery: The ideology of social hierarchies between hunters and herders. Journal of Anthropological Archaeology 17(2): 201215.

Smith, A.B. (2005). African Herders: Emergence of Pastoral Traditions. Walnut Creek: AltaMira Press.

Smith, A.B. (2006). Ideological inhibitors to hunters becoming food producers in Africa. Calgary, AB: Unpublished paper presented at the $18^{\text {th }}$ biennial conference of the Society of Africanist Archaeologists, June 23-26.

Smith, A.B, Sadr, K., Gribble, J. \& Yates, R. (1991). Excavations in the south-western Cape, South Africa, and the archaeological identity of prehistoric hunter gatherers within the last 2000 years. South African Archaeological Bulletin 47: 62-64.

Smith, A., Malherbe, C., Guenther, M., \& Berens, P. (2000). The Bushmen of Southern Africa: A foraging society in transition. Cape Town, SA: David Philip Publishers.

Smith, B. (2006). Reading rock art and writing genetic history: regionalism, ethnicity and the rock art of southern Africa. In The Prehistory of Africa: Tracing the Lineage of Modern Man, ed. H. Soodyall, pp. 76-94. Jeppestown: Jonathan Ball.

Smith, B.H. (1984). Patterns of molar wear in hunter-gatherers and agriculturalists. American Journal of Physical Anthropology: 63: 39-56. 
Smith, E.A. \& Winterhalder, B. (1992). Natural selection and decision making: Some fundamental principals. In Evolutionary Ecology and Human Behaviour, eds. E.A. Smith and B. Winterhalder, pp. 25-60. New York: Aldine de Gruyter.

Smith, H.F., Terhune, C.E., \& Lockwood, C.A. (2007). Genetic, geographic, and environmental correlates of human temporal bone variation. American Journal of Physical Anthropology 134: 312-322.

Smith P., Horwitz, L.K., \& Kaplan, E. (1992). Skeletal evidence for population change in the Late Holocene of the south-western Cape: A radiological study. South African Archaeological Bulletin 47: 82-88.

Sokal, R.R. \& F.J. Rohlf. (1995). Biometry, $3^{\text {rd }}$ Edition. New York: W.H. Freeman and Company.

Solway, J.S., \& Lee, R.B. (1990). Foragers, genuine or spurious? Situating the Kalahari San in history. Current Anthropology 31(2): 109-122.

Sparks, C.S., \& Jantz, R.L. (2002). A reassessment of human cranial plasticity: Boas revisited. Proceedings of the National Academy of Sciences United States of America 99: 14636-14639.

Spence, M.W. (1974). Residential practices and the distribution of skeletal traits in Teotihuacan, Mexico. Man 9: 262-273.

Steadman, D.W. (1998). The population shuffle in the central Illinois valley: a diachronic model of Mississippian biocultural interactions. World Archaeology 30(2): 306-326.

Steadman, D.W. (2001). Mississippians in motion? A population genetic analysis of interregional gene flow in west-central Illinois. American Journal of Physical Anthropology 114: 61-73.

Steckel, R.H. \& Rose, J.C. (2002). The Backbone of History: health and nutrition in the Western Hemisphere. New York: Cambridge University Press.

Stirland, A.J. (1998). Musculoskeletal evidence for activity: Problems of evaluation. International Journal of Osteoarchaeology 8: 354-362.

Stock, J.T. (2002). Climatic and Behavioural Influences on Postcranial Robusticity Among Holocene Foragers. Ph.D. dissertation, University of Toronto. 
Stock, J.T. (2006). Hunter-gatherer postcranial robusticity relative to patterns of mobility, climatic adaptation, and selection for tissue economy. American Journal of Physical Anthropology 131: 194-204.

Stock, J. \& Pfeiffer, S. (2001). Linking the structural variability in long bone diaphyses to habitual behaviors: foragers from southern African Later Stone Age and the Andaman Islands. American Journal of Physical Anthropology 115(4): 337-348.

Stock, J.T. \& Pfeiffer, S. (2004). Long bone robusticity and subsistence behaviour among Later Stone Age foragers of the forest and fynbos biomes of South Africa. Journal of Archaeological Science 31: 999-1013.

Stojanowski, C.M. (2005). Biocultural Histories in La Florida: A Bioarchaeological Perspective. Tuscaloosa: University of Alabama Press.

Stojanowski, C.M, Larsen, C.S., Tung, T.A., \& McEwan, B.G. (2007). Biological structure and health implications from tooth size at Mission San Luis de Apalachee. American Journal of Physical Anthropology 132: 207-222.

Stojanowski, C.M. \& Schillaci, M.A. (2006). Phenotypic approaches for understanding patterns of intracemetery biological variation. Yearbook of Physical Anthropology 49: 49-88.

Stoneking, M. (2006). Genetic evidence for our recent African ancestry. In The Prehistory of Africa: Tracing the Lineage of Modern Man, ed. H. Soodyall, pp. 21-30. Johannesburg: Jonathan Ball Publishers.

Stynder, D.D. (2006). A quantitative assessment of variation in Holocene Khoesan crania from South Africa's western, south-western, southern, and south-eastern coasts and coastal forelands. PhD Dissertation. University of Cape Town, Cape Town, South Africa.

Stynder, D.D., Rogers Ackerman, R., \& Sealy, J.C. (2007a). Craniofacial variation and population continuity during the South African Holocene. American Journal of Physical Anthropology 134: 489-500.

Stynder, D.D., Rogers Ackerman, R., \& Sealy, J.C. (2007b). Early to mid-Holocene South African Later Stone Age human crania exhibit a distinctly Khoesan morphological pattern. South African Journal of Science 103: 349-352. 
Suchey, J. \& Katz, D. (1986). Skeletal age standards derived from an extensive multiracial sample of modern Americans. American Journal of Physical Anthropology 69: 269.

Tafuri, M.A. \& Di Lernia, S. (2006). Consuming food, embodying places: Dietary and social resources in the Holocene Acacus Mountains (Libyan Sahara). Calgary, AB: Unpublished paper presented at the $18^{\text {th }}$ biennial conference of the Society of Africanist Archaeologists, June 23-26.

Thomas, D.H. (1992). Archaeology Down to Earth, $2^{\text {nd }}$ Edition. Fort Worth, TX: Harcourt Brace College Publishers.

Tobias, P. (1962). On the increasing stature of Bushmen. Anthropos 57: 801-810.

Trinkaus, E., Churchill, S.E., \& Ruff, C.B. (1994). Postcranial robusticity in Homo: Humeral bilateral asymmetry and bone plasticity. American Journal of Physical Anthropology 93:1-34.

Underhill, P.A., Shen, P., Lin, A.A., Jin, L., Passarino, G., Yang, W.H., Auffman, E., Bonne-Tamir, B., Bertranpetit, J., Francalacci, P., Ibrahim, M., Jenkins, T., Kidd, J.R., Mehdi, S.Q., Seielstad, M.T., Wells, R.S., Piazza, A., Davis, R.W., Feldman, M.W., Cavalli-Sforza, L.L., \& Oefner, P.J. (2000). Y chromosome sequence variation and the history of human populations. Nature Genetics 26:358-361.

van Reenen, J.F. (1966). Dental features of a low-caries primitive population. Journal of Dental Research 45: 703-713.

van Reenen, J. F. (1982). The effects of attrition on tooth dimension s of San (Bushmen). In Teeth: Form, Function and Evolution, ed. B. Kurten, pp. 182-203. New York: Columbia University Press.

Vansina, J. (1995). New linguistic evidence and 'the Bantu' expansion'. Journal of African History 36: 173-195.

Vansina, J. (1996). A Slow Revolution: Farming in Subequatorial Africa. In The Growth of Farming Communities in Africa from the Equator Southwards, ed. J.E.G. Sutton, pp. 15-26. Azania special volume XXIX-XXX, The British Institute in Eastern Africa. Van Valen, L. (1973). Body size and the number of plants and animals. Evolution 27: 27-35. Vigilant, L., Stoneking, M., Harpending, H., Hawkes, K., \& Wilson, A.C. (1991). African populations and the evolution of human mitochondrial DNA. Science 253: 15031507. 
Vogel, J., Plug, I., \& Webley, L. (1997). New dates for the introduction of sheep into South Africa: The evidence from Spoegrivier Cave in Namaqualand. South African Journal of Science 93: 246-248.

Walker, N.J. (1983). The significance of an early date for pottery and sheep in Zimbabwe. South African Archaeological Bulletin 38: 88-92.

Walker, R.S., \& Hamilton, M. (2008). Life-history consequences of density dependence and the evolution of human body sizes. Current Anthropology 49(1): 115-122.

Webley, L. (1992). Early evidence for sheep from Spoegrivier cave, Namaqualand. Southern African Field Archaeology 1:3-13.

Widlok, T. (1999). Living on Mangetti: 'Bushman' Autonomy and Namibian Independence. Oxford Studies in Social and Cultural Anthropology. Oxford: Oxford University Press.

Wiley, A.S. (2005). Does milk make children grow? Relationships between milk consumption and height in NHANES 1999-2002. American Journal of Human Biology 17: 425-441.

Williams-Blangero, S., \& Blangero, J. (1989). Anthropometric variation and the genetic structure of the Jirels of Nepal. Human Biology 61(1): 1-12.

Williams-Blangero, S., \& Blangero, J. (1990). Effects of population structure on withingroup variation in the Jirels of Nepal. Human Biology 62(1): 131-146.

Williamson, R.F. \& Pfeiffer, S. (2003). Bones of the Ancestors: The Archaeology and Osteobiography of the Moatfield Ossuary. Mercury Series Archaeology Paper 163. Gatineau: Canadian Museum of Civilization.

Wilson, M.L., \& Lundy, J.K. (1994). Estimated living statures of dated Khoisan skeletons from the south-western coastal region of South Africa. South African Archaeological Bulletin 49: 2-8.

Winterhalder, B. (1981). Optimal foraging strategies and hunter-gatherer research in anthropology: Theory and models. In Hunter Gatherer Foraging Strategies: Ethnographic and Archaeological Analyses, eds. B. Winterhalder and E.A. Smith, pp. 13-35. Chicago, IL: University of Chicago Press.

Winterhalder, B. \& Smith, E.A. (1981). New perspectives on Hunter-Gatherer Socioecology. In Hunter Gatherer Foraging Strategies: Ethnographic and 
Archaeological Analyses, eds. B. Winterhalder and E.A. Smith, pp. 1-12. Chicago, IL: University of Chicago Press.

Woodburn, J. (1982). Egalitarian societies. Man 17:431-451. 
Appendix 1. Map of the Eastern Cape Region displaying some of the burial locations of the skeletal remains studied in this research.

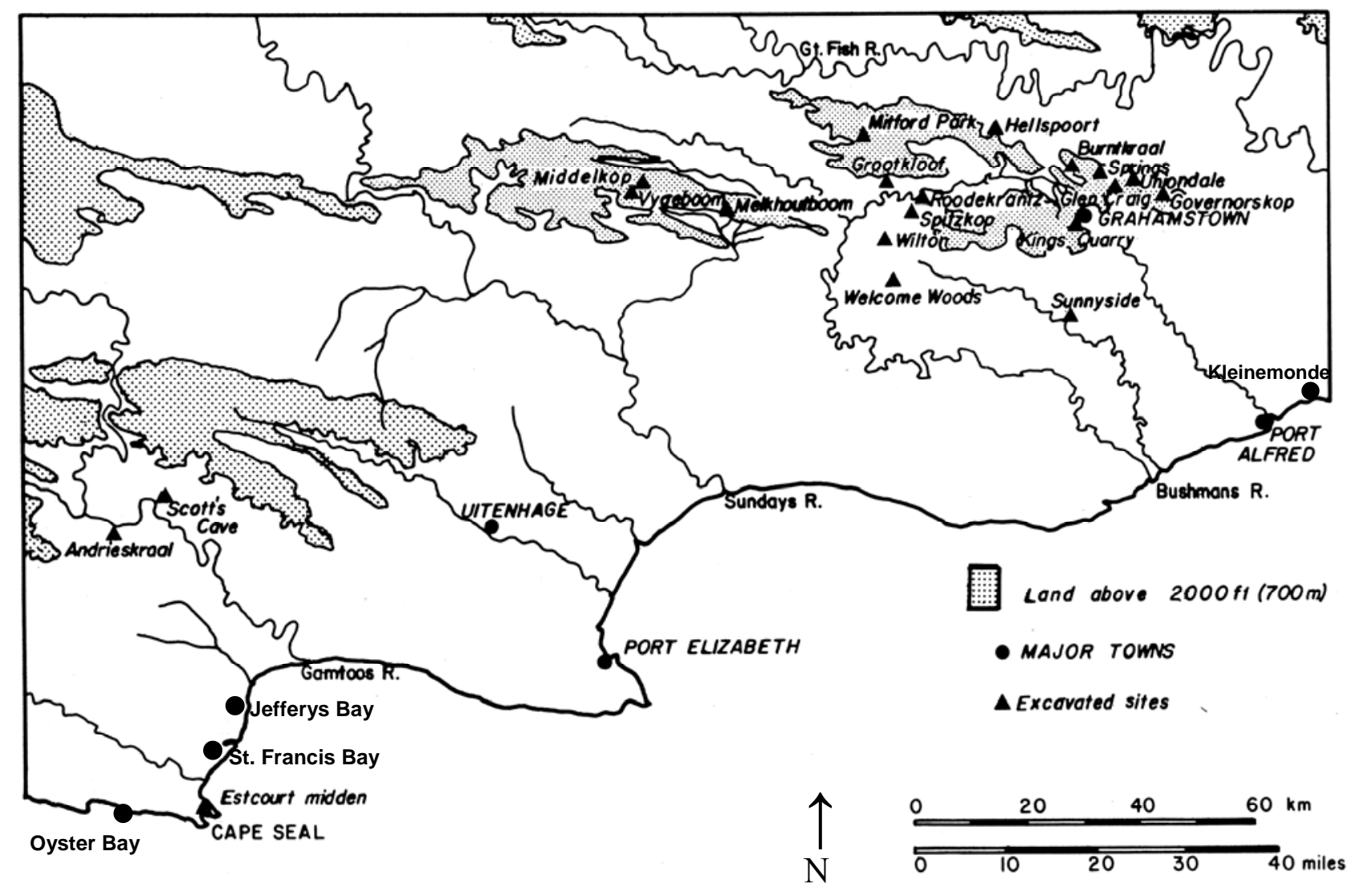

(Adapted from Deacon 1976) 
Appendix 2. Total Eastern Cape Skeletal Sample

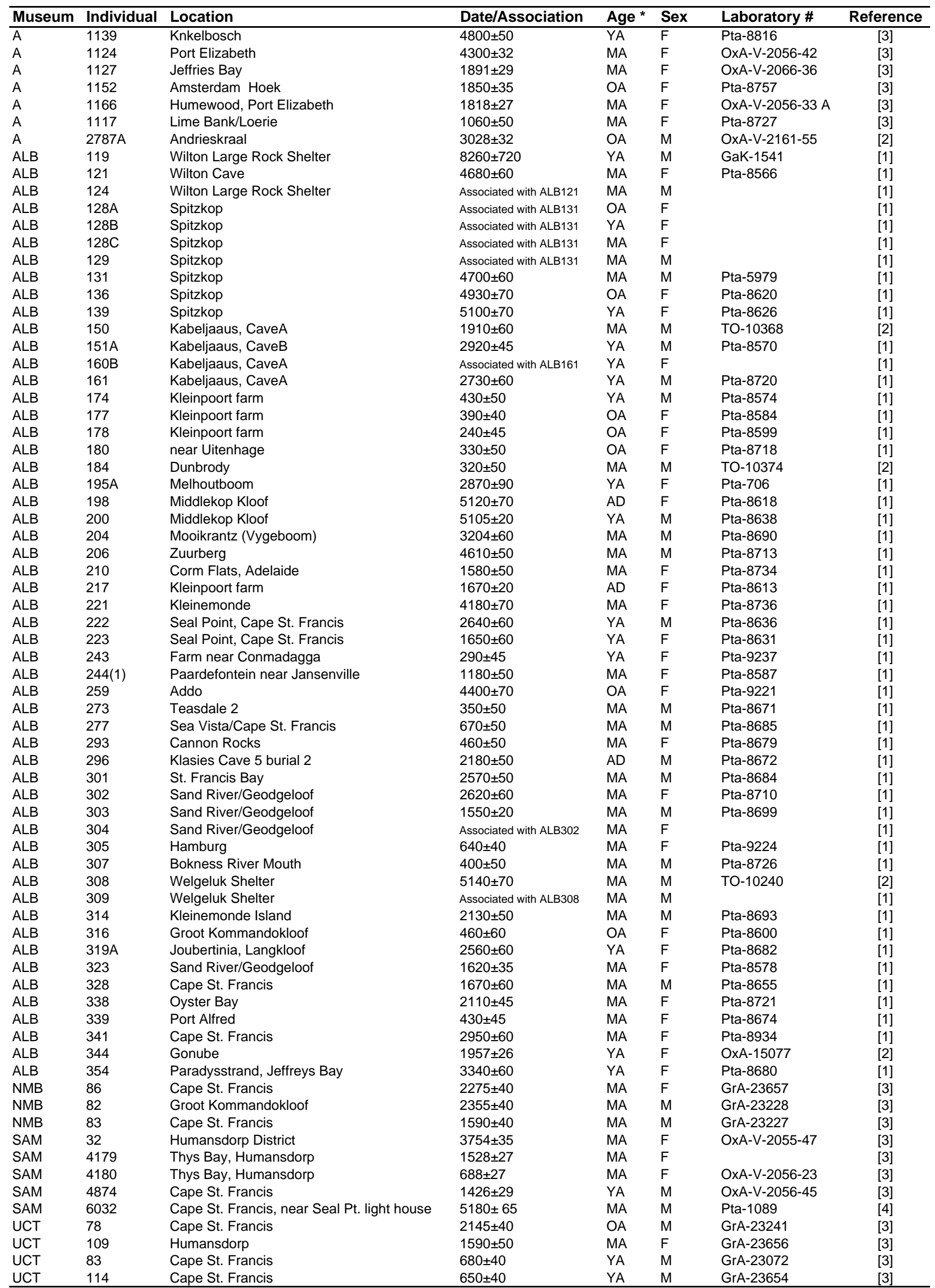

* Age: AD = Adolescent (16-20 years); YA = Young Adult (21-35 years); MA = Middle Adult (36-49 years); OA = Old Adult (50+ years) References: [1] Sealy, pers. comm.; [2] Pfeiffer, pers. Comm.; [3] Stynder (2006); [4] Morris (1992) 
Appendix 3. List of Craniometric Varaibles Examined in this Study

\begin{tabular}{|c|c|c|c|}
\hline Measurement & Code & Source & Definition \\
\hline \multicolumn{4}{|l|}{ CRANIUM } \\
\hline Maximum Cranial Length & GOL & Buikstra and Ubelaker (1994) & glabella (g) to opisthocranion (op) \\
\hline Nasion Occipital Length & NOL & Howells (1973) & nasion (n) to opisthocranion (op) \\
\hline Cranial Base Length & BNL & Buikstra and Ubelaker (1994) & basion (ba) to nasion (n) \\
\hline Basion Bregma Height & $\mathrm{BBH}$ & Buikstra and Ubelaker (1994) & basion (ba) to bregma (b) \\
\hline Maximum Cranial Breadth & $\mathrm{XCB}$ & Buikstra and Ubelaker (1994) & eurion (eu) to eurion (eu) \\
\hline Bistephanic breadth & STB & Howells (1973) & intersection coronal suture and inferior temporal \\
\hline Minimum frontal breadth & WFB & Buikstra and Ubelaker (1994) & frontotemporale (ft) to frontotemporale (ft) \\
\hline Bizygomatic breadth & ZYB & Buikstra and Ubelaker (1994) & zygion (zy) to zygion (zy) \\
\hline Biauricular breadth & AUB & Buikstra and Ubelaker (1994) & auriculare $(\mathrm{au})$ to auriculare $(\mathrm{au})$ \\
\hline Biasterionic breadth & ASB & Howells (1973) & point where temporal, occipital and parietal meet \\
\hline Basion-prosthion length & BPL & Buikstra and Ubelaker (1994) & basion (ba) to prosthion (pr) \\
\hline Nasion-prosthion length & $\mathrm{NPH}$ & Howells (1973) & nasion $(\mathrm{n})$ to prosthion $(\mathrm{pr})$ \\
\hline Nasal height & NLH & Buikstra and Ubelaker (1994) & nasion (n) to nasospinale (ns) \\
\hline Bijugal breadth & JUB & Howells (1973) & breadth across malars \\
\hline Nasal breadth & NLB & Buikstra and Ubelaker (1994) & alare (al) to alare (al) \\
\hline Maxillo-Alveolar breadth & MAB & Buikstra and Ubelaker (1994) & ectomolare $(\mathrm{ecm})$ to ectomolare $(\mathrm{ecm})$ \\
\hline Maxillo-Alveolar length & MAL & Buikstra and Ubelaker (1994) & prosthion (pr) to alveolon (alv) \\
\hline Mastoid Length & $\mathrm{MDH}$ & Buikstra and Ubelaker (1994) & $\begin{array}{l}\text { upper border of external auditory meatus to inferior } \\
\text { tip mastoid process }\end{array}$ \\
\hline Orbital Height & $\mathrm{OBH}$ & Buikstra and Ubelaker (1994) & $\begin{array}{l}\text { direct distance between superior and inferior orbital } \\
\text { margins }\end{array}$ \\
\hline Orbital Breadth & OBB & Buikstra and Ubelaker (1994) & dakryon (d) to ectoconchion (ec) \\
\hline Interorbital breadth & DKB & Buikstra and Ubelaker (1994) & dacryon $(d)$ to dacryon $(d)$ \\
\hline Naso-Dacryl Subtense & NDS & Howells (1973) & $\begin{array}{l}\text { when taking interorbital measure, lower co-ordinate } \\
\text { arm to deepest point nasal profile }\end{array}$ \\
\hline Minimum nasal breadth & WNB & Howells (1973) & minimum breath of nasal bones \\
\hline Simiotic breadth & SIS & Howells (1973) & $\begin{array}{l}\text { when taking minimum nasal breadth, lower co- } \\
\text { ordinate arm to deepest point }\end{array}$ \\
\hline Bimaxillary Breadth & ZMB & Howells (1973) & zygomaxillare (zm) to zygomaxillare (zm) \\
\hline $\begin{array}{l}\text { Subspinale to Bimaxillary } \\
\text { Breadth Subtense }\end{array}$ & SSS & Howells (1973) & $\begin{array}{l}\text { when taking bimaxillary breadth, lower co-ordintae } \\
\text { arm to deepest point below nasal spine }\end{array}$ \\
\hline Bifrontal Breadth & FMB & Howells (1973) & $\begin{array}{l}\text { frontomalare anterior (fma) to frontomalare anterior } \\
\text { (fma) }\end{array}$ \\
\hline Upper Facial Breadth & UFBR & Buikstra and Ubelaker (1994) & $\begin{array}{l}\text { frontomalare temporale (fmt) to frontomalare } \\
\text { temporale (fmt) }\end{array}$ \\
\hline Naso-Frontal Subtense & NAS & Howells (1973) & subtense from nasion to bifrontal breadth \\
\hline Biorbital Breadth & EKB & Howells (1973) & ectoconchion (ec) to ectoconchion (ed) \\
\hline Dacryon Subtense & DKS & Howells (1973) & mean subtensen from dacryon (d) to biorbital \\
\hline Malar Length, Inferior & IML & Howells (1973) & $\begin{array}{l}\text { zygomaxillare }(\mathrm{zm}) \text { anterior to lowest point } \\
\text { zygotemporal suture }\end{array}$ \\
\hline Malar Length, Maximum & $\mathrm{XML}$ & Howells (1973) & lower zygotemporal suture to zygoorbitale \\
\hline Malar Subtense & MLS & Howells (1973) & $\begin{array}{l}\text { when taking maximum malar length, drop co- } \\
\text { ordinate arm to area of zygomaticofacial foramen }\end{array}$ \\
\hline Cheek Height & WMH & Howells (1973) & $\begin{array}{l}\text { minimum distance from lower border of orbit to } \\
\text { lower margin of maxilla }\end{array}$ \\
\hline Glabella Projection & GLS & Howells (1973) & subtense from nasion $(\mathrm{n})$ to supraglabellare \\
\hline Frontal Chord & FRC & Buikstra and Ubelaker (1994) & nasion $(n)$ to bregma $(b)$ \\
\hline Frontal Subtense & FRS & Howells (1973) & subtense nasion (n) to bregma (b) \\
\hline Frontal Fraction & FRF & Howells (1973) & fraction nasion (n) to bregma (b) \\
\hline Parietal Chord & PAC & Buikstra and Ubelaker (1994) & bregma (b) to lambda (I) \\
\hline Parietal Subtense & PAS & Howells (1973) & subtense bregma (b) to lambda (I) \\
\hline Parietal Fraction & PAF & Howells (1973) & fraction bregma (b) to lambda (I) \\
\hline Occipital Chord & OCC & Buikstra and Ubelaker (1994) & lambda (I) to opisthion (o) \\
\hline Occipital Subtense & OCS & Howells (1973) & subtense lambda (I) to opisthion (o) \\
\hline Occipital Fraction & OCF & Howells (1973) & fraction lambda (I) to opisthion (o) \\
\hline Foramen Magnum Length & FOL & Buikstra and Ubelaker (1994) & basion (b) to opisthion (o) \\
\hline Foramen Magnum Breadth & FOB & Buikstra and Ubelaker (1994) & $\begin{array}{l}\text { greatest distance between lateral margins of } \\
\text { foramen magnum }\end{array}$ \\
\hline \multicolumn{4}{|l|}{ MANDIBLE } \\
\hline Chin Height & GNI & Buikstra and Ubelaker (1994) & \multirow{2}{*}{$\begin{array}{l}\text { infradentale (id) to gnathion (gn) } \\
\text { alveolar process to inferior mandibular border at } \\
\text { mental foramen }\end{array}$} \\
\hline Height of Mandibular Body & HML & Buikstra and Ubelaker (1994) & \\
\hline Breadth of Mandibular Body & TML & Buikstra and Ubelaker (1994) & \multirow{7}{*}{$\begin{array}{l}\text { maximum breadth in region of mental foramen } \\
\text { gonion (go) to goinion (go) } \\
\text { condylon laterale (cdl) to condylon laterale (cdl) } \\
\text { least breadth of ramus perpendicular to height } \\
\text { distance from hightest point on the condyle to } \\
\text { gonion (go) } \\
\text { anterior margin of chin to posterior border of } \\
\text { mandibular angle } \\
\text { angle formed by inferior border of corpus and } \\
\text { posterior border of ramus }\end{array}$} \\
\hline Bigonial Width & GOG & Buikstra and Ubelaker (1994) & \\
\hline Bicondylar Breadth & CDL & Buikstra and Ubelaker (1994) & \\
\hline Minimum Ramus Breadth & WRB & Buikstra and Ubelaker (1994) & \\
\hline Maximum Ramus Height & $\mathrm{XRH}$ & Buikstra and Ubelaker (1994) & \\
\hline Mandibular Length & XML-M & Buikstra and Ubelaker (1994) & \\
\hline Mandibular Angle & MAN & Buikstra and Ubelaker (1994) & \\
\hline
\end{tabular}


Appendix 4. List of Odontometric Variables (Maximum and Cervical Dimensions) Examined in this Study

\begin{tabular}{|c|c|c|}
\hline Tooth & Measurement & Code \\
\hline \multicolumn{3}{|l|}{ MAXILLARY } \\
\hline \multirow[t]{4}{*}{ First Incisor } & Maximum buccolingual diameter & UI1MBL \\
\hline & Maximum mesiodistal diameter & UI1MMD \\
\hline & Cervical buccolingual diameter & UI1CBL \\
\hline & Cervical mesiodistal diameter & UI1CMD \\
\hline \multirow[t]{4}{*}{ Second Incisor } & Maximum buccolingual diameter & UI2MBL \\
\hline & Maximum mesiodistal diameter & UI2MMD \\
\hline & Cervical buccolingual diameter & UI2CBL \\
\hline & Cervical mesiodistal diameter & UI2CMD \\
\hline \multirow[t]{4}{*}{ Canine } & Maximum buccolingual diameter & UCMBL \\
\hline & Maximum mesiodistal diameter & UCMMD \\
\hline & Cervical buccolingual diameter & UCCBL \\
\hline & Cervical mesiodistal diameter & UCCMD \\
\hline \multirow[t]{4}{*}{ First Premolar } & Maximum buccolingual diameter & UP1MBL \\
\hline & Maximum mesiodistal diameter & UP1MMD \\
\hline & Cervical buccolingual diameter & UP1CBL \\
\hline & Cervical mesiodistal diameter & UP1CMD \\
\hline \multirow[t]{4}{*}{ Second Premolar } & Maximum buccolingual diameter & UP2MBL \\
\hline & Maximum mesiodistal diameter & UP2MMD \\
\hline & Cervical buccolingual diameter & UP2CBL \\
\hline & Cervical mesiodistal diameter & UP2CMD \\
\hline \multirow[t]{4}{*}{ First Molar } & Maximum buccolingual diameter & UM1MBL \\
\hline & Maximum mesiodistal diameter & UM1MMD \\
\hline & Cervical buccolingual diameter & UM1CBL \\
\hline & Cervical mesiodistal diameter & UM1CMD \\
\hline \multirow[t]{4}{*}{ Second Molar } & Maximum buccolingual diameter & UM2MBL \\
\hline & Maximum mesiodistal diameter & UM2MMD \\
\hline & Cervical buccolingual diameter & UM2CBL \\
\hline & Cervical mesiodistal diameter & UM2CMD \\
\hline \multirow[t]{4}{*}{ Third Molar } & Maximum buccolingual diameter & UM3MBL \\
\hline & Maximum mesiodistal diameter & UM3MMD \\
\hline & Cervical buccolingual diameter & UM3CBL \\
\hline & Cervical mesiodistal diameter & UM3CMD \\
\hline \multicolumn{3}{|l|}{ MANDIBULAR } \\
\hline \multirow[t]{4}{*}{ First Incisor } & Maximum buccolingual diameter & LI1MBL \\
\hline & Maximum mesiodistal diameter & LI1MMD \\
\hline & Cervical buccolingual diameter & LI1CBL \\
\hline & Cervical mesiodistal diameter & LIICMD \\
\hline \multirow[t]{4}{*}{ Second Incisor } & Maximum buccolingual diameter & LI2MBL \\
\hline & Maximum mesiodistal diameter & LI2MMD \\
\hline & Cervical buccolingual diameter & LI2CBL \\
\hline & Cervical mesiodistal diameter & LI2CMD \\
\hline \multirow[t]{4}{*}{ Canine } & Maximum buccolingual diameter & LCMBL \\
\hline & Maximum mesiodistal diameter & LCMMD \\
\hline & Cervical buccolingual diameter & LCCBL \\
\hline & Cervical mesiodistal diameter & LCCMD \\
\hline \multirow[t]{4}{*}{ First Premolar } & Maximum buccolingual diameter & LP1MBL \\
\hline & Maximum mesiodistal diameter & LP1MMD \\
\hline & Cervical buccolingual diameter & LP1CBL \\
\hline & Cervical mesiodistal diameter & LP1CMD \\
\hline Second Premolar & Maximum buccolingual diameter & LP2MBL \\
\hline & Maximum mesiodistal diameter & LP2MMD \\
\hline & Cervical buccolingual diameter & LP2CBL \\
\hline & Cervical mesiodistal diameter & LP2CMD \\
\hline First Molar & Maximum buccolingual diameter & LM1MBL \\
\hline & Maximum mesiodistal diameter & LM1MMD \\
\hline & Cervical buccolingual diameter & LM1CBL \\
\hline & Cervical mesiodistal diameter & LM1CMD \\
\hline Second Molar & Maximum buccolingual diameter & LM2MBL \\
\hline & Maximum mesiodistal diameter & LM2MMD \\
\hline & Cervical buccolingual diameter & LM2CBL \\
\hline & Cervical mesiodistal diameter & LM2CMD \\
\hline Third Molar & Maximum buccolingual diameter & LM3MBL \\
\hline & Maximum mesiodistal diameter & LM3MMD \\
\hline & Cervical buccolingual diameter & LM3CBL \\
\hline & Cervical mesiodistal diameter & LM3CMD \\
\hline
\end{tabular}


Appendix 5. List of Post Cranial Metric Variables Examined in this Study

\begin{tabular}{|c|c|c|}
\hline Bone & Measurement & Code \\
\hline \multirow[t]{4}{*}{ Clavicle } & Maximum Length & CLAVXL \\
\hline & Anterior-Posterior Diameter & CLAVAPD \\
\hline & Superior-Inferior Diameter & CLAVSID \\
\hline & Clavical Robusticity Index & CLAVRBSTINDX \\
\hline \multirow{6}{*}{ Scapula } & Maximum Height & SCAPXHT \\
\hline & Maximum Breadth & SCAPXBR \\
\hline & Spine Length & SCAPSPIL \\
\hline & Glenoid Breadth & SCAPGCBR \\
\hline & Glenoid Height & SCAPGCH \\
\hline & Scapular Index & SCAPINDX \\
\hline \multirow[t]{8}{*}{ Humerus } & Maximum Length & HUMXL \\
\hline & Maximum Midshaft Diameter & HUMMIDXD \\
\hline & Minimum Midshaft Diameter & HUMMIDND \\
\hline & Humeral Midshaft Index & HMMIDSHFTINDX \\
\hline & Head Diameter & HUMHEAD \\
\hline & Epicondylar Breadth & HUMEPBR \\
\hline & Humeral Robusticity Index & HUMRBSTINDX \\
\hline & Clavicular-Humeral Index & CLAHUMINDX \\
\hline \multirow[t]{6}{*}{ Radius } & Maximum Length & RADXL \\
\hline & Maximum Head Diameter & RADHED \\
\hline & Anterior-Posterior Diameter at Midshaft & RADAPD \\
\hline & Medial-Lateral Diameter at Midshaft & RADMLD \\
\hline & Radial Shaft Index & RADSHFTINDX \\
\hline & Brachial Index & RADHUMINDX \\
\hline \multirow[t]{7}{*}{ Ulna } & Maximum Length & ULNAXL \\
\hline & Physiological Length & ULNAPHYL \\
\hline & Maximum Olecranon Breadth & ULNAXOBR \\
\hline & Minimum Olecranon Breadth & ULNANOBR \\
\hline & Anterior-Posterior Shaft Diameter & ULNAAPD \\
\hline & Medial-Lateral Shaft Diameter & ULNAMLD \\
\hline & Ulnar Shaft Index & ULNASHFTINDX \\
\hline \multirow[t]{5}{*}{ Sacrum } & Anterior Height & SACRANTH \\
\hline & Anterior Breadth & SACRANTB \\
\hline & S1 Breadth & SACRSIBR \\
\hline & Skeletal Trunk Height & SKTRNKHT \\
\hline & Sacral Index & SACR INDX \\
\hline \multirow[t]{6}{*}{ Innominate } & Innominate Height & INNOHT \\
\hline & Iliac Breadth & ILIACBR \\
\hline & Pubis Length & PUBISL \\
\hline & Ischium Length & ISCHIUML \\
\hline & Bi-iliac Breadth & BIILIACBR \\
\hline & Ischopubic Index & ISCHPUBINDX \\
\hline \multirow[t]{15}{*}{ Femur } & Maximum Length & FEMXL \\
\hline & Bicondylar Length & FEMBL \\
\hline & Subtrochanteric Anterior-Posterior Diameter & FEMSTAPD \\
\hline & Subtrochanteri Medial-Lateral Diameter & FEMSTMLD \\
\hline & Platymeric Index & PLATYMINDX \\
\hline & Midshaft Anterior-Posterior Diameter & FEMMIDADL \\
\hline & Midshaft Medial-Lateral Diameter & FEMMIDMD \\
\hline & Femoral Midshaft Index & FEMMIDSHFTINDX \\
\hline & Vertical Head Diameter & FEMVHEDD \\
\hline & Horizontal Head Diameter & FEMHHEDD \\
\hline & Lateral Condyle Anterior-Posterior Diameter & FEMLCAPD \\
\hline & Medial Condyle Anterior-Posterior Diameter & FEMMCAPD \\
\hline & Epicondylar Breadth & FEMEPICB \\
\hline & Midshaft Circumference & FEMMIDCR \\
\hline & Femoral Robusticty Index & FEMRBSTINDX \\
\hline \multirow[t]{8}{*}{ Tibia } & Maximum Length & TIBXL \\
\hline & Proximal Epiphyseal Breadth & TIBPEPBR \\
\hline & Distal Epiphyseal Breadth & TIBDEPBR \\
\hline & Anterior-Posterior Diameter at Nutrient Foramen & TIBAPNFD \\
\hline & Medial-Lateral Diameter at Nutrient Foramen & TIBMLNFD \\
\hline & Platycnemic Index & PLATYCNINDX \\
\hline & Circumference Nutrient Foramen & TIBNUTFC \\
\hline & Crural Index & TIBFEMINDX \\
\hline \multirow[t]{2}{*}{ Fibula } & Maximum Length & FIBXL \\
\hline & Maximum Diameter at Midshaft & FIBMSXD \\
\hline \multirow[t]{2}{*}{ Calcaneus } & Maximum Length & CALCXLN \\
\hline & Middle Breadth & CALCMBR \\
\hline 1st Metatarsal & Maximum Length & MT1XL \\
\hline & Anterior-Posterior Diameter of Base & MTIAP \\
\hline & Medial-Lateral Diameter of Base & MTIML \\
\hline
\end{tabular}




\begin{tabular}{|c|c|c|c|c|c|c|c|}
\hline Trait & Code & Score & Location & Trait & Code & Score & Location \\
\hline Facial & & & & Basilar & & & \\
\hline Infraorbital Suture & IOSUT & $\begin{array}{l}0=\text { absent } \\
1=\text { present (all forms) } \\
9=\text { unobservable }\end{array}$ & Left and Right & Condylar canal absent & CONDCANABS & $\begin{array}{l}0=\text { absent } \\
1=\text { present } \\
9=\text { unobservable }\end{array}$ & Left and Right \\
\hline Infraorbital Foramen & IOFORAM & $\begin{array}{l}0=\text { absent } \\
1=\text { division (partial-complete) } \\
9=\text { unobservable }\end{array}$ & Left and Right & Divided hypoglossal canal & DIHYPOC & $\begin{array}{l}0=\text { absent } \\
1=\text { present }(\text { trace-complete) } \\
9=\text { unobservable }\end{array}$ & Left and Right \\
\hline Zygomaticofacial foramen & ZYFFOR & $\begin{array}{l}0=\text { absent } \\
1=\text { present }(\text { any number }) \\
9=\text { unobservable }\end{array}$ & Left and Right & Tympanic dehiscence & TYMDIHS & $\begin{array}{l}0=\text { absent } \\
1=\text { present }(\text { any defect }) \\
9=\text { unobservable }\end{array}$ & Left and Right \\
\hline Partial Metopic Suture & METOPPART & $\begin{array}{l}0=\text { absent } \\
1=\text { present }(\text { nasal or bregma) } \\
9=\text { unobservable }\end{array}$ & Midline & Marginal Foramen & MARGFOR & $\begin{array}{l}0=\text { absent } \\
1=\text { present } \\
9=\text { unobservable }\end{array}$ & Left and Right \\
\hline Complete Metopic Suture & METOPFULL & $\begin{array}{l}0=\text { absent } \\
1=\text { present } \\
9=\text { unobservable }\end{array}$ & Midline & Foramen spinosum incomplete & FRSPINI & $\begin{array}{l}0=\text { absent } \\
1=\text { present }(\text { partial-complete }) \\
9=\text { unobservable }\end{array}$ & Left and Right \\
\hline Supraorbital notch & SONOTCH & $\begin{array}{l}0=\text { absent } \\
1=\text { present } \\
9=\text { unobservable }\end{array}$ & Left and Right & Foramen ovale incomplete & FOROVLI & $\begin{array}{l}0=\text { absent } \\
1=\text { present }(\text { partial-complete) } \\
9=\text { unobservable }\end{array}$ & Left and Right \\
\hline Supraorbital foramen & SOFORAM & $\begin{array}{l}0=\text { absent } \\
1=\text { present }(\text { medial } 1 / 2 \text { orbit) } \\
9=\text { unobservable }\end{array}$ & Left and Right & Pterygospinous bridge - trace & PTSBRTR & $\begin{array}{l}0=\text { absent } \\
1=\text { present } \\
9=\text { unobservable }\end{array}$ & Left and Right \\
\hline Supratrochlear notch & STNOTCH & $\begin{array}{l}0=\text { absent } \\
1=\text { present } \\
9=\text { unobservable }\end{array}$ & Left and Right & Pterygospinous bridge - partial & PTSBRPT & $\begin{array}{l}0=\text { absent } \\
1=\text { present } \\
9=\text { unobservable }\end{array}$ & Left and Right \\
\hline Frontal Foramen & FRONTFOR & $\begin{array}{l}0=\text { absent } \\
1=\text { present }(\text { lateral } 1 / 2 \text { orbit }) \\
9=\text { unobservable }\end{array}$ & Left and Right & Pterygospinous bridge - complete & PTSBRCOM & $\begin{array}{l}0=\text { absent } \\
1=\text { present } \\
9=\text { unobservable }\end{array}$ & Left and Right \\
\hline Trochlear Spur & TROCSPR & $\begin{array}{l}0=\text { absent } \\
1=\text { present } \\
9=\text { unobservable }\end{array}$ & Left and Right & Pterygospinous bridge - all & PTSBRALL & $\begin{array}{l}0=\text { absent } \\
1=\text { present } \\
9=\text { unobservable }\end{array}$ & Left and Right \\
\hline Superior & & & & Pterygoalar bridge - trace & PTABRGTR & $0=$ absent & Left and Right \\
\hline Frontal Lines & FRONTLN & $\begin{array}{l}0=\text { absent } \\
1=\text { present }\end{array}$ & Left and Right & & & $\begin{array}{l}1=\text { present } \\
9=\text { unobservable }\end{array}$ & \\
\hline Coronal Ossicle & CORONL & $\begin{array}{l}9=\text { unobservable } \\
0=\text { absent } \\
1=\text { present }\end{array}$ & Left and Right & Pterygoalar bridge - partial & PTABRGPT & $\begin{array}{l}0=\text { absent } \\
1=\text { present } \\
9=\text { unobservable }\end{array}$ & Left and Right \\
\hline Bregmatic bone & BREGMATB & $\begin{array}{l}9=\text { unobservable } \\
0=\text { absent } \\
1=\text { present }\end{array}$ & Midline & Pterygoalar bridge - complete & BTABRGCOM & $\begin{array}{l}0=\text { absent } \\
1=\text { present } \\
9=\text { unobservable }\end{array}$ & Left and Right \\
\hline Sagittal ossicle & SATITOSSIC & $\begin{array}{l}9=\text { unobservable } \\
0=\text { absent } \\
1=\text { present }\end{array}$ & Midline & Pterygoalar bridge - all & PTABRGALL & $\begin{array}{l}0=\text { absent } \\
1=\text { present } \\
9=\text { unobservable }\end{array}$ & Left and Right \\
\hline Parietal Foramen & PFORAM & $\begin{array}{l}9=\text { unobservable } \\
0=\text { absent }(\text { any location }) \\
1=\text { present }\end{array}$ & Left and Right & Ossified Apical Ligament & APICOSS & $\begin{array}{l}0=\text { absent } \\
1=\text { present } \\
9=\text { unobservable }\end{array}$ & Midline \\
\hline & & $9=$ unobservable & & Palatine torus development & PALTOR & $0=a b s e n t$ & Midline \\
\hline $\begin{array}{l}\text { Lateral } \\
\text { Asterionic hone }\end{array}$ & & & & & & $1=$ present (any expression) & \\
\hline Asterionic bone & ASTRINB & $\begin{array}{l}0=\text { absent } \\
1=\text { present }\end{array}$ & Left and Right & Posterior & & $9=$ unobservable & \\
\hline Ossicle occipitomastoid suture & OMSUT & $\begin{array}{l}9=\text { unobservable } \\
0=\text { absent } \\
1=\text { present }\end{array}$ & Left and Right & Apical bone & APICALBN & $\begin{array}{l}0=\text { absent } \\
1=\text { present } \\
9=\text { unobservable }\end{array}$ & Midline \\
\hline Parietal notch bone & PARNOTB & $\begin{array}{l}9=\text { unobservable } \\
0=\text { absent } \\
1=\text { present }\end{array}$ & Left and Right & Inca Bone & INCABONE & $\begin{array}{l}0=\text { absent } \\
1=\text { present } \\
9=\text { unobservable }\end{array}$ & Midline \\
\hline Parietal process of temporal & PARPROC & $\begin{array}{l}9=\text { unobservable } \\
0=\text { absent } \\
1=\text { present }\end{array}$ & Left and Right & Lambdoid Ossicle & LAMBOSS & $\begin{array}{l}0=\text { absent } \\
1=\text { present } \\
9=\text { unobservable }\end{array}$ & Left and Right \\
\hline Auditory exostosis & AUDEXOS & $\begin{array}{l}9=\text { unobservable } \\
0=\text { absent } \\
1=\text { present } \\
9=\text { unobservable }\end{array}$ & Left and Right & $\begin{array}{l}\text { Mandible } \\
\text { Mylohyoid bridge development }\end{array}$ & MYLHBRD & $\begin{array}{l}0=\text { absent } \\
1=\text { present (partial-complete) } \\
9=\text { unobservable }\end{array}$ & Left and Right \\
\hline Mastoid foramen temporal & MASTFRTEMP & $\begin{array}{l}0=\text { absent } \\
1=\text { present }(\text { any number) } \\
9=\text { unobservable }\end{array}$ & Left and Right & Mental foramen absent & MENTFORABS & $\begin{array}{l}0=\text { absent } \\
1=\text { present } \\
9=\text { unobservable }\end{array}$ & Left and Right \\
\hline Mastoid foramen sutural & MASTFRSUT & $\begin{array}{l}0=\text { absent } \\
1=\text { present }(\text { any number }) \\
9=\text { unobservable }\end{array}$ & Left and Right & Accessory mental foramen & ACCMENTFOR & $\begin{array}{l}0=\text { absent }(\text { any number }) \\
1=\text { present } \\
9=\text { unobservable }\end{array}$ & Left and Right \\
\hline Mastoid foramen occipital & MASTFROCC & $\begin{array}{l}0=\text { absent } \\
1=\text { present }(\text { any number) } \\
9=\text { unobservable }\end{array}$ & Left and Right & Mandibular torus & MANDTOR & $\begin{array}{l}0=\text { absent } \\
1=\text { present }(\text { any expression }) \\
9=\text { unobservable }\end{array}$ & Left and Right \\
\hline Epipteric bone & EPITER & $\begin{array}{l}0=\text { absent } \\
1=\text { present } \\
9=\text { unobservable }\end{array}$ & Left and Right & & & & \\
\hline Os Japonicom & OSJAP & $\begin{array}{l}0=\text { absent } \\
1=\text { present }(\text { trace-complete) } \\
9=\text { unobservable }\end{array}$ & Left and Right & & & & \\
\hline
\end{tabular}




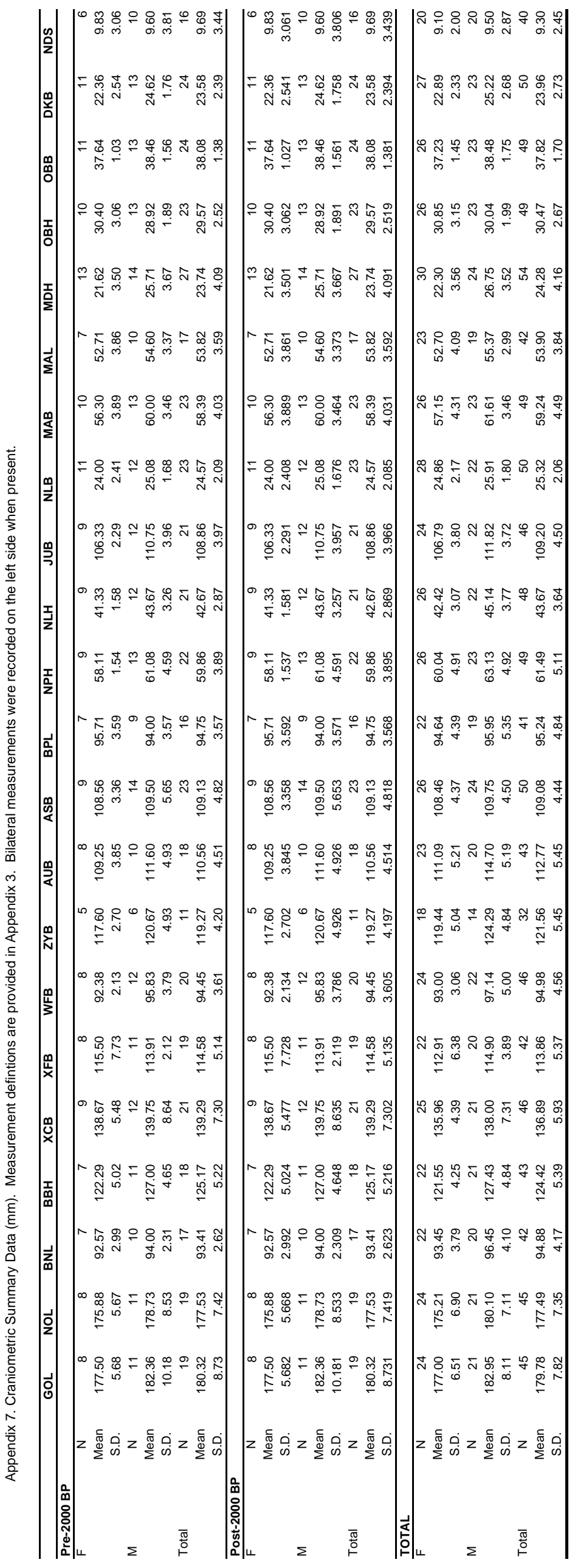




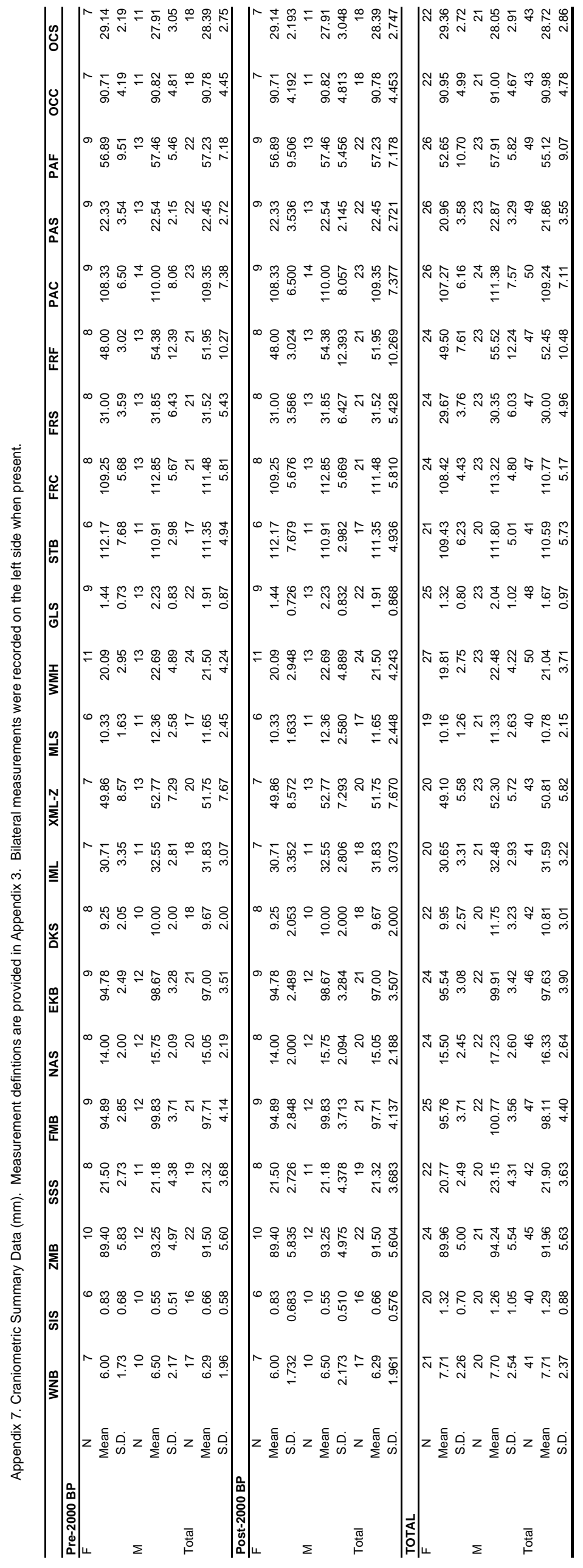




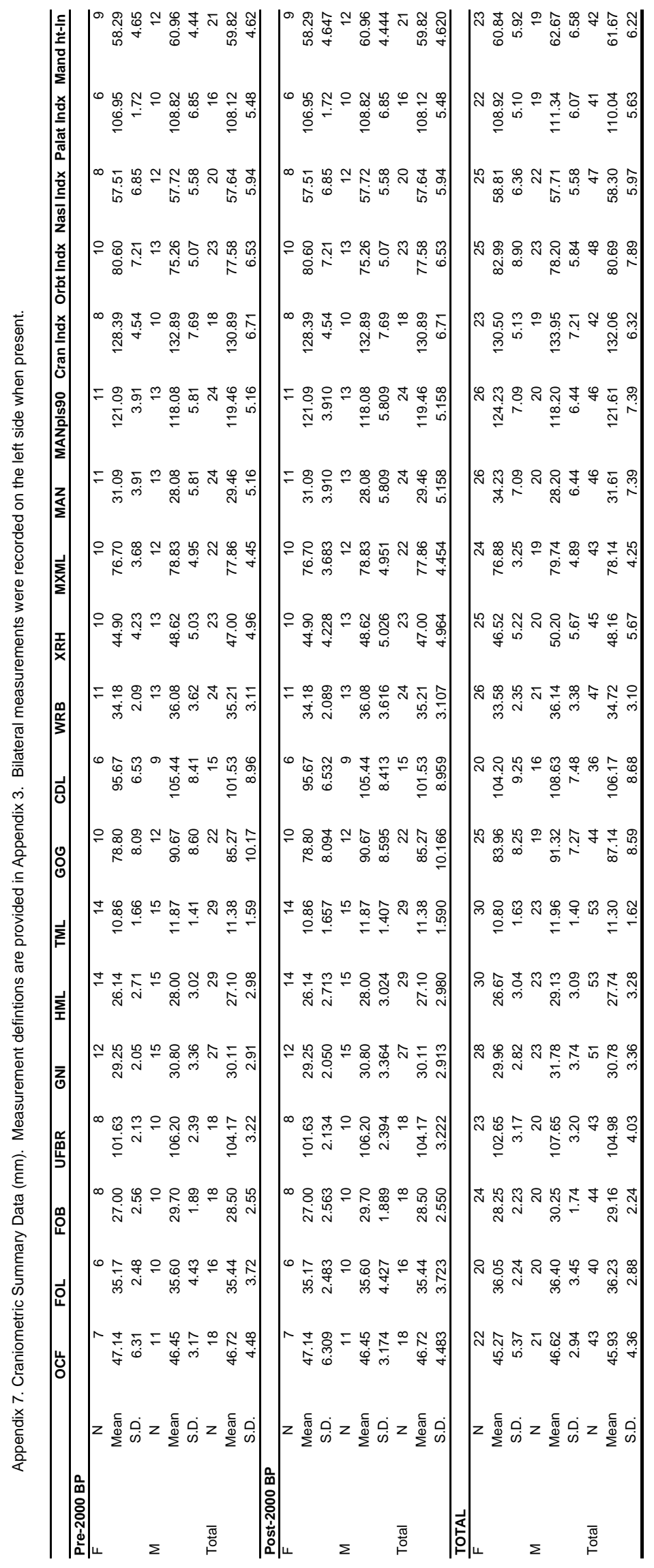




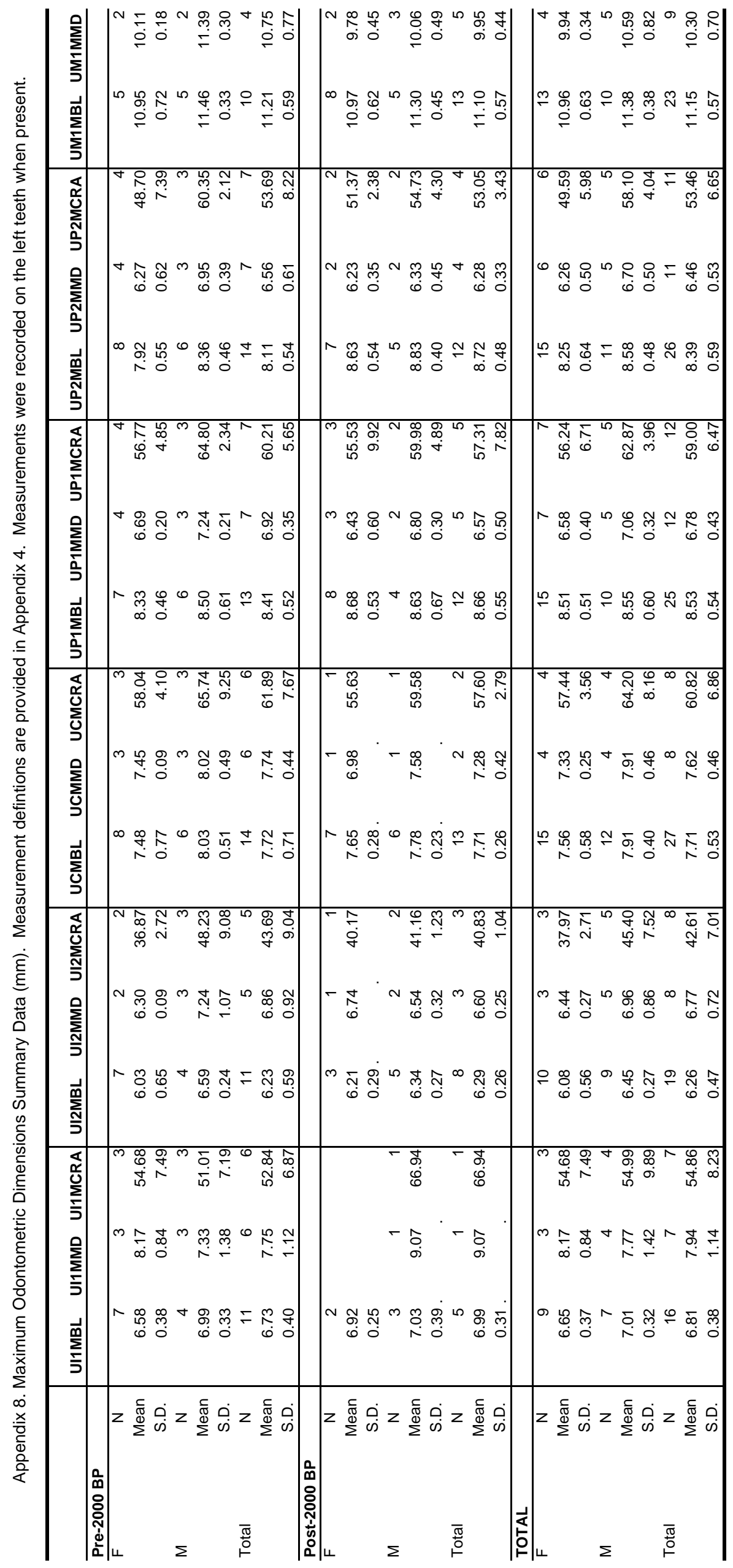




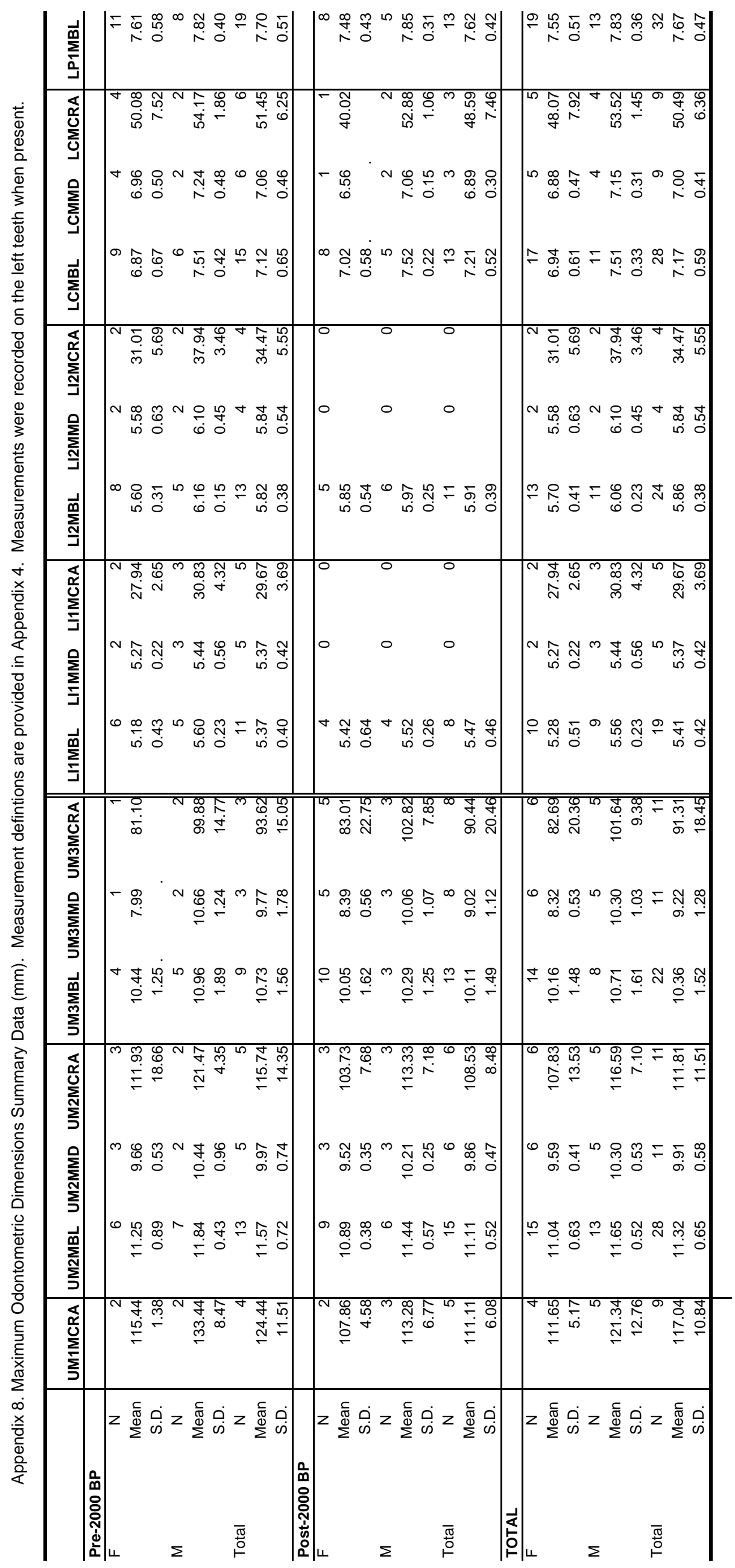




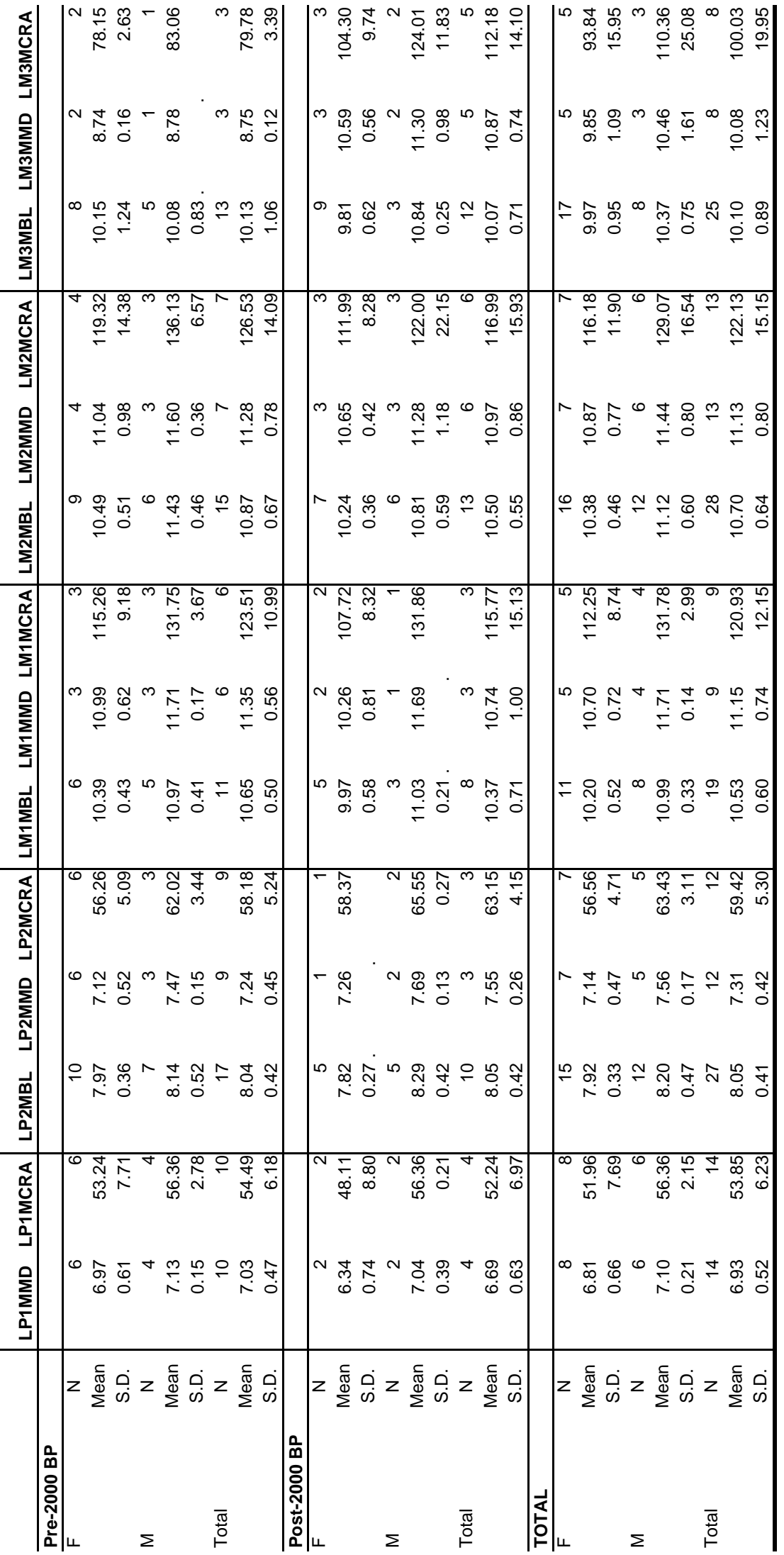




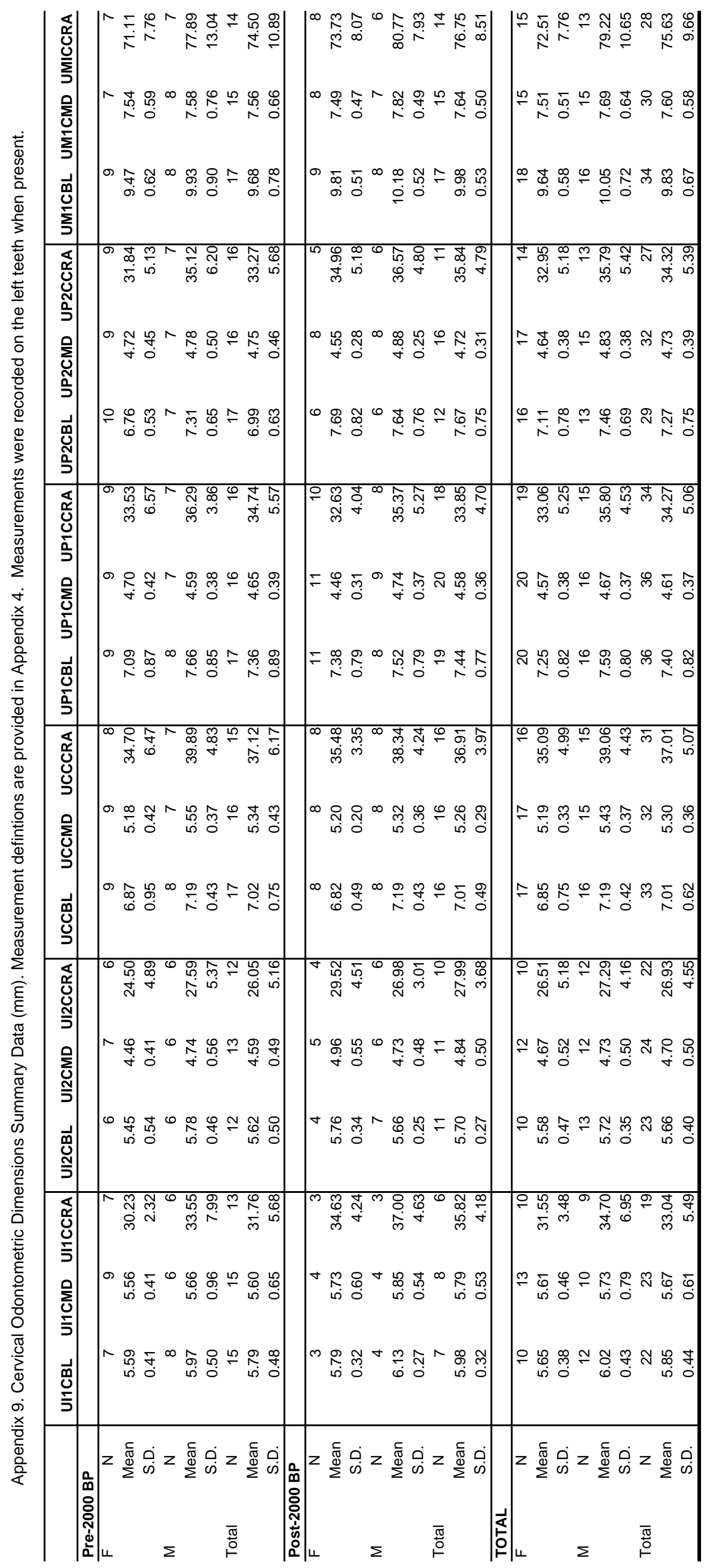




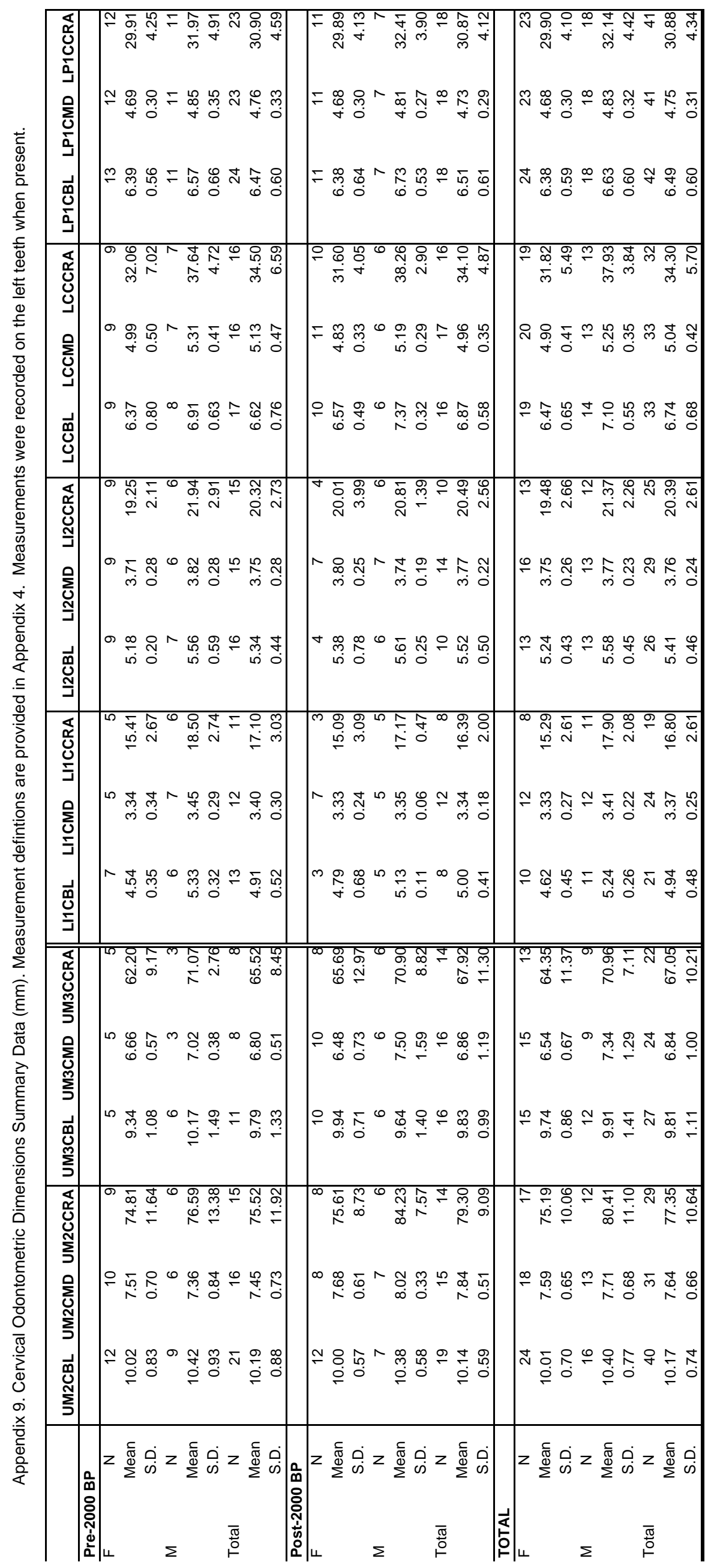




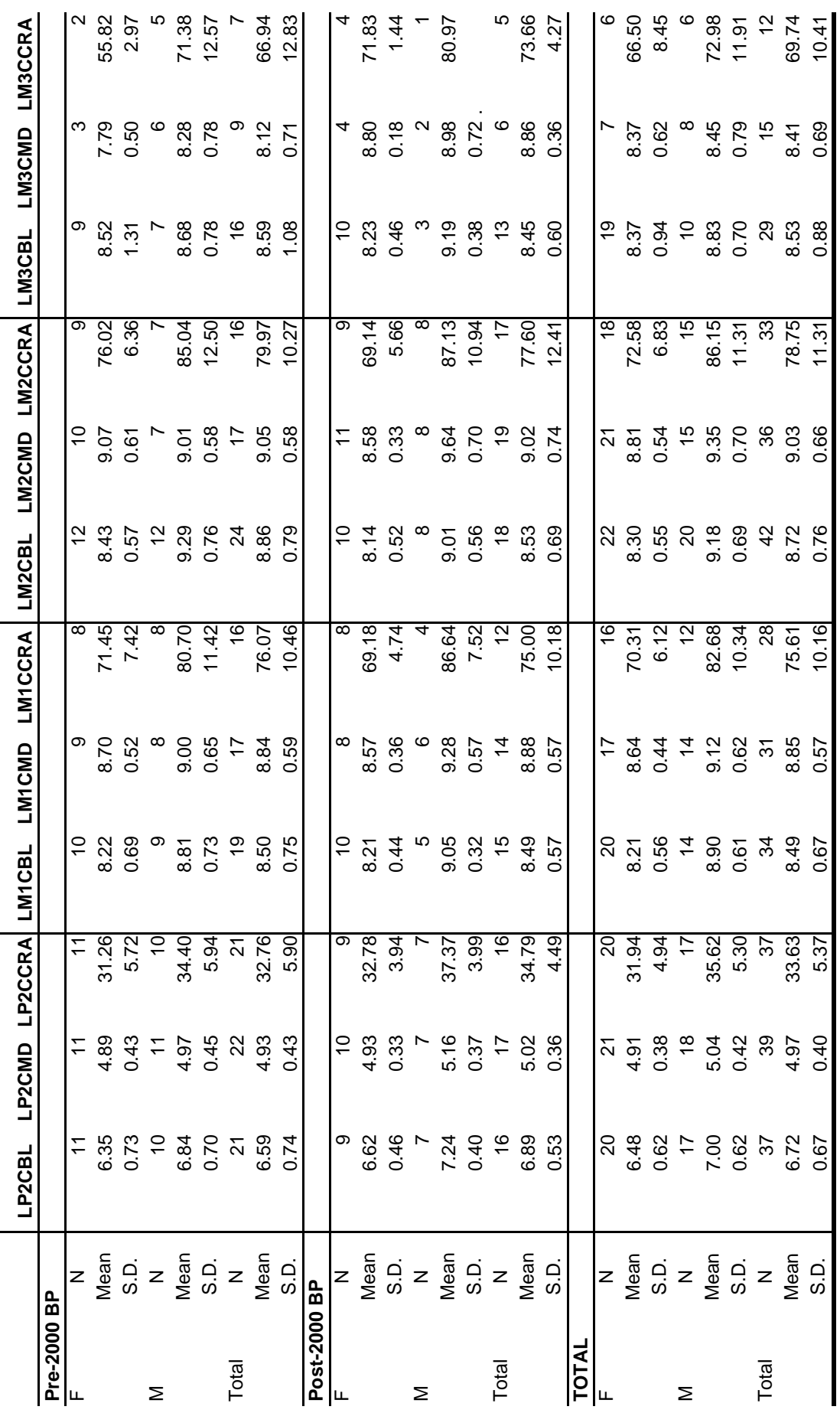




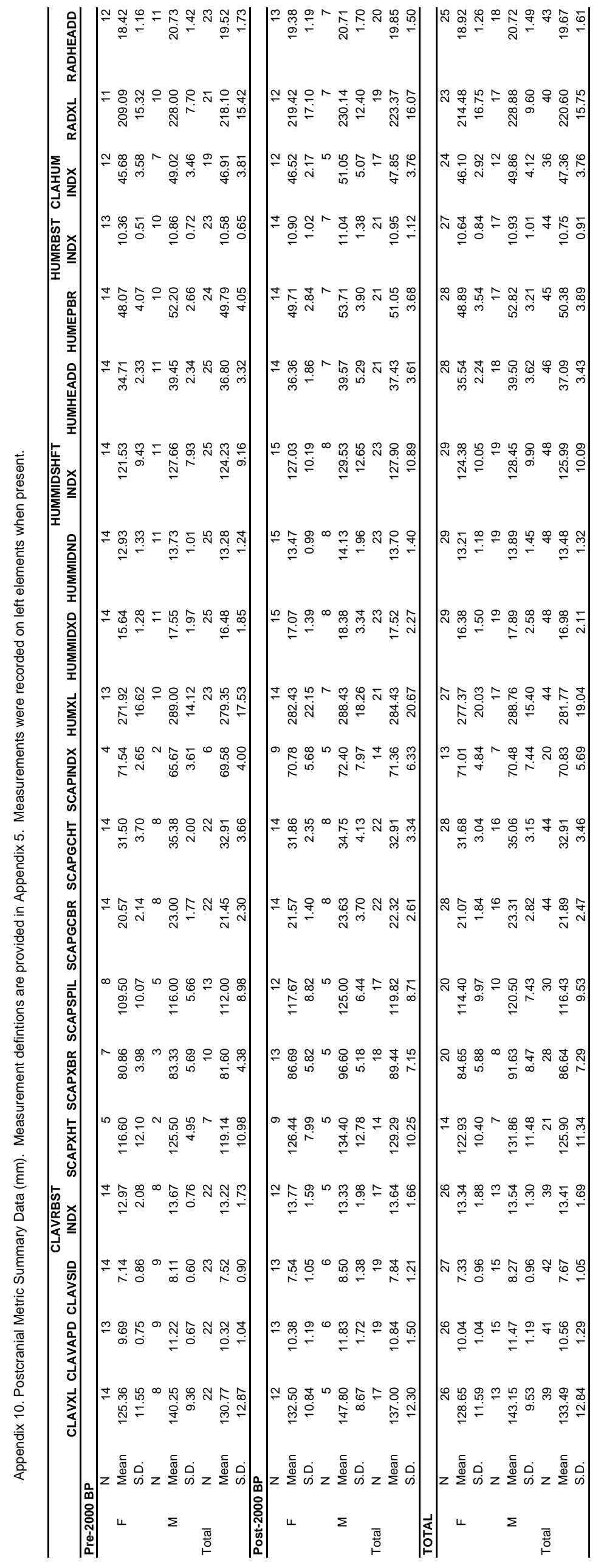




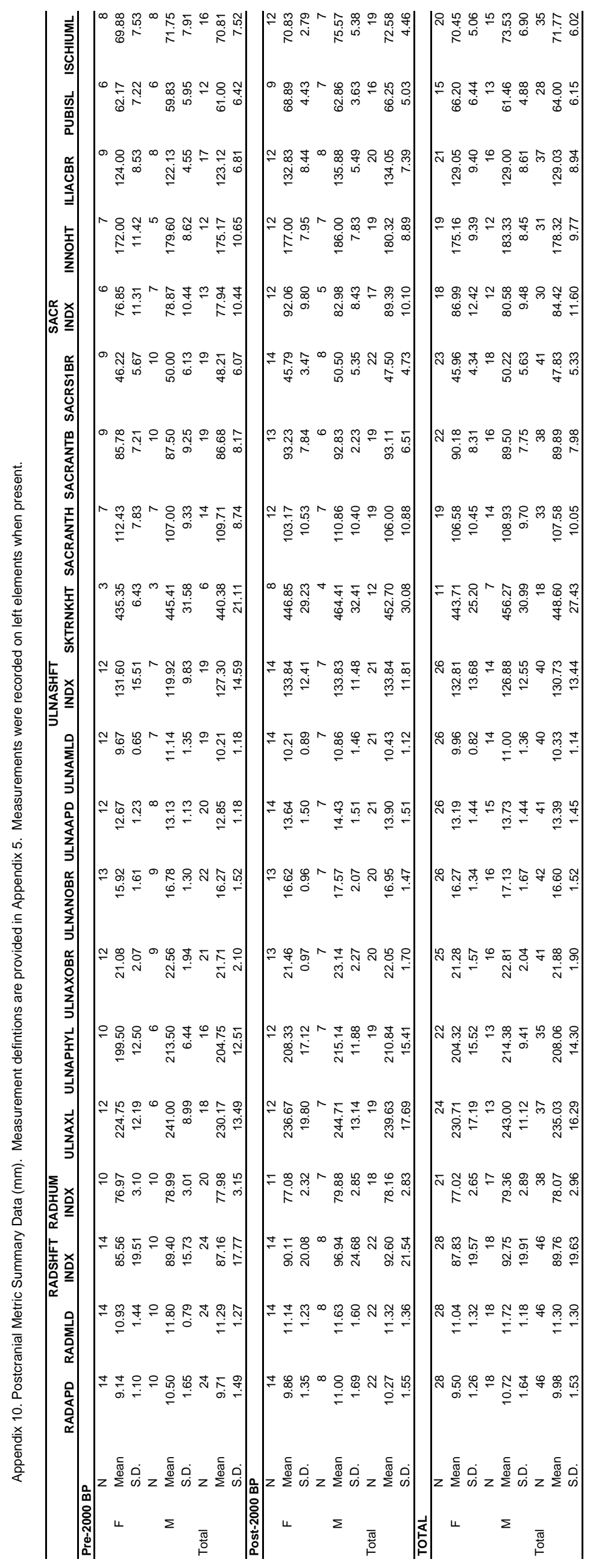




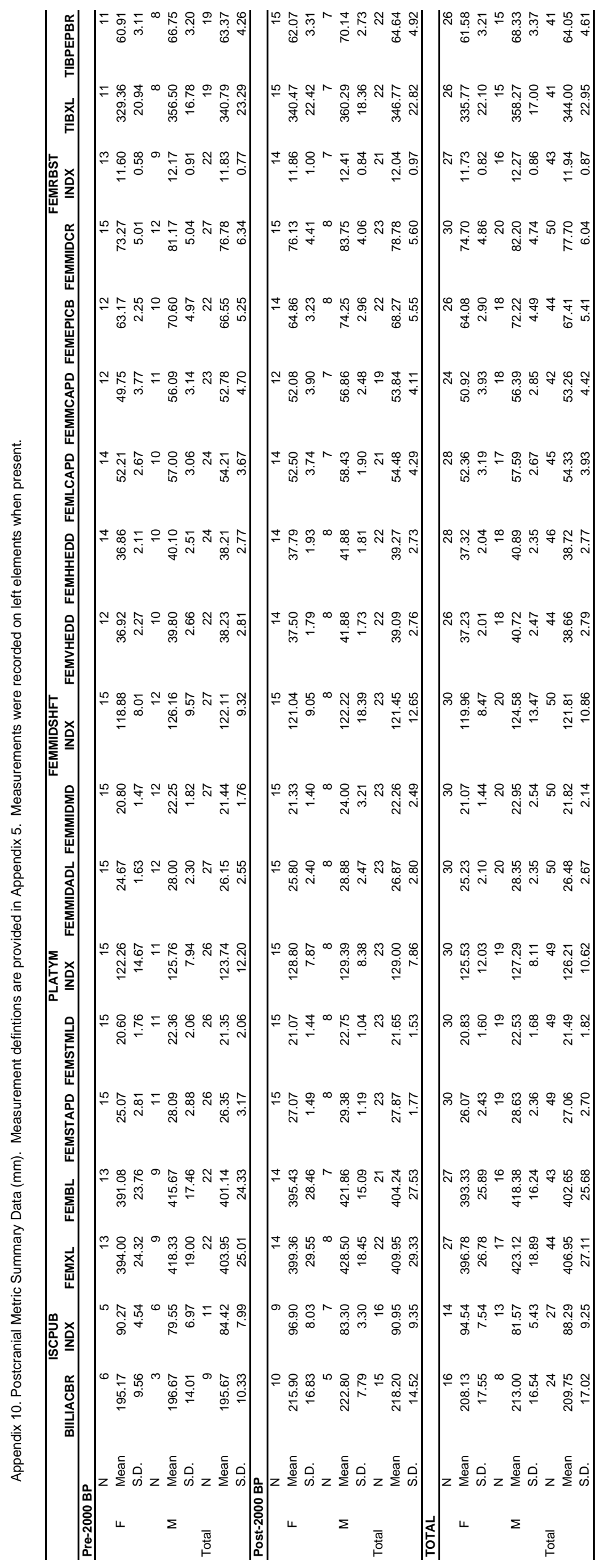




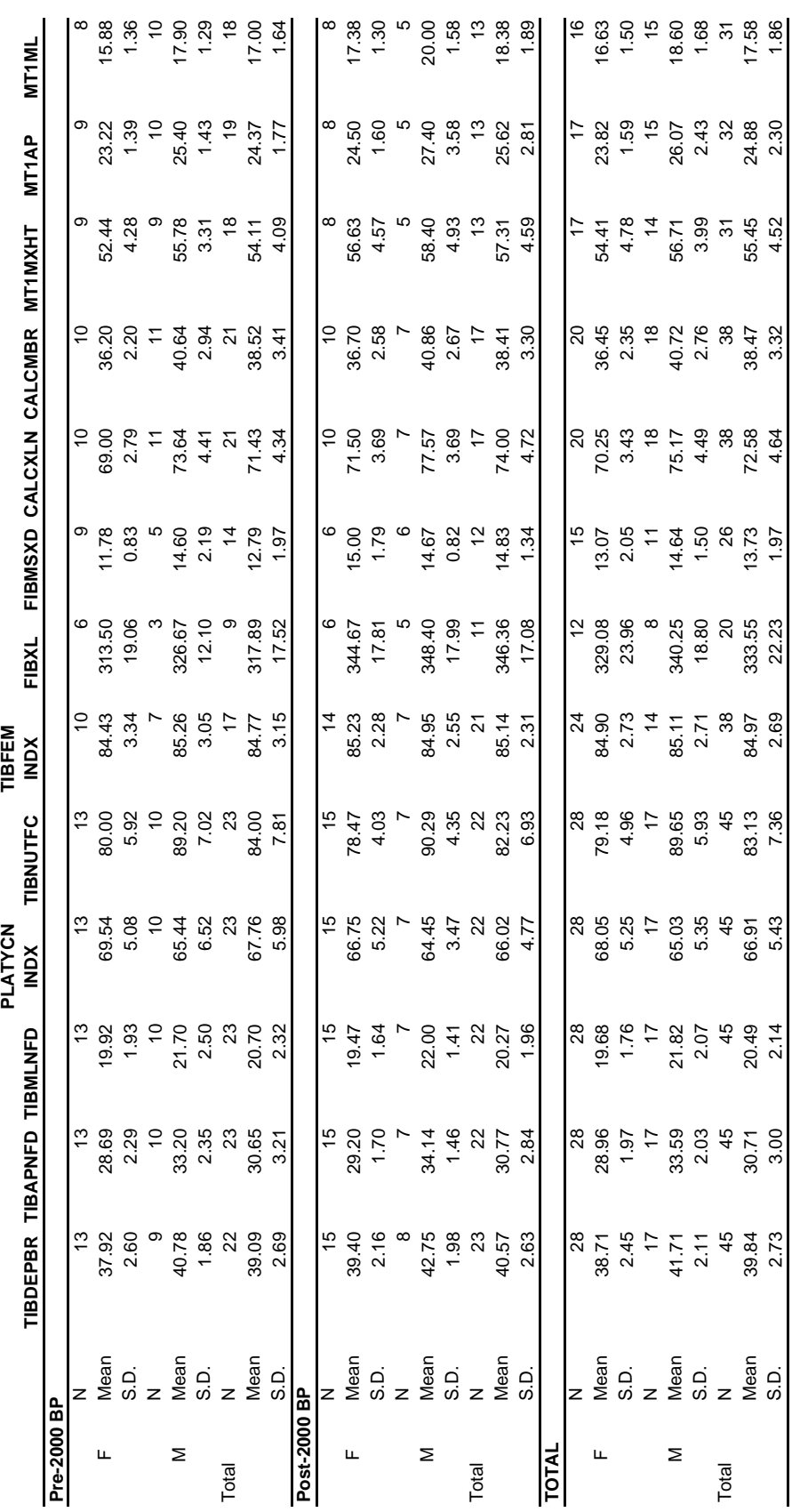


Appendix 11. Kolmogorov-Smirnov test of normality for craniometric variables with Monte Carlo approximations

\begin{tabular}{|c|c|c|c|c|c|c|}
\hline \multicolumn{7}{|c|}{$\begin{array}{ll}\text { Pre-2000 BP Females } \\
\end{array}$} \\
\hline \multirow[b]{3}{*}{ Variable } & \multirow[b]{3}{*}{$\mathrm{N}$} & \multirow[b]{3}{*}{ K-S Z } & \multirow[b]{3}{*}{$\mathrm{p}$} & \multirow[b]{3}{*}{$\mathrm{p}$} & \multicolumn{2}{|l|}{ Monte Carlo } \\
\hline & & & & & \multicolumn{2}{|c|}{ 95\% C.I. } \\
\hline & & & & & Lower Bound & Upper Bound \\
\hline GOL & 8 & 0.806 & 0.534 & $.451(\mathrm{c})$ & 0.441 & 0.460 \\
\hline NOL & 8 & 1.074 & 0.199 & $.149(\mathrm{c})$ & 0.142 & 0.156 \\
\hline BNL & 7 & 0.448 & 0.988 & $.967(\mathrm{c})$ & 0.963 & 0.970 \\
\hline $\mathrm{BBH}$ & 7 & 0.678 & 0.748 & $.657(\mathrm{c})$ & 0.647 & 0.666 \\
\hline XCB & 9 & 0.662 & 0.774 & $.691(\mathrm{c})$ & 0.682 & 0.700 \\
\hline XFB & 8 & 0.634 & 0.816 & $.735(\mathrm{c})$ & 0.726 & 0.744 \\
\hline WFB & 8 & 0.783 & 0.572 & $.485(c)$ & 0.476 & 0.495 \\
\hline ZYB & 5 & 0.355 & 1.000 & $.996(\mathrm{c})$ & 0.995 & 0.998 \\
\hline AUB & 8 & 0.842 & 0.478 & $.392(c)$ & 0.382 & 0.401 \\
\hline ASB & 9 & 0.658 & 0.780 & $.699(\mathrm{c})$ & 0.690 & 0.708 \\
\hline BPL & 7 & 0.775 & 0.585 & $.486(\mathrm{c})$ & 0.476 & 0.496 \\
\hline $\mathrm{NPH}$ & 9 & 0.489 & 0.971 & $.938(\mathrm{c})$ & 0.934 & 0.943 \\
\hline $\mathrm{NLH}$ & 9 & 0.417 & 0.995 & $.983(\mathrm{c})$ & 0.981 & 0.986 \\
\hline JUB & 9 & 0.633 & 0.818 & $.744(\mathrm{c})$ & 0.735 & 0.752 \\
\hline NLB & 11 & 0.754 & 0.621 & $.543(\mathrm{c})$ & 0.533 & 0.553 \\
\hline MAB & 10 & 0.560 & 0.912 & $.860(\mathrm{c})$ & 0.854 & 0.867 \\
\hline MAL & 7 & 0.779 & 0.578 & $.480(\mathrm{c})$ & 0.470 & 0.490 \\
\hline $\mathrm{MDH}$ & 13 & 0.493 & 0.968 & $.938(c)$ & 0.933 & 0.943 \\
\hline $\mathrm{OBH}$ & 10 & 0.580 & 0.890 & $.832(\mathrm{c})$ & 0.825 & 0.839 \\
\hline OBB & 11 & 0.911 & 0.377 & $.314(\mathrm{c})$ & 0.305 & 0.323 \\
\hline DKB & 11 & 0.867 & 0.441 & $.374(\mathrm{c})$ & 0.365 & 0.383 \\
\hline NDS & 6 & 1.079 & 0.194 & $.133(c)$ & 0.126 & 0.140 \\
\hline WNB & 7 & 0.567 & 0.905 & $.845(c)$ & 0.838 & 0.852 \\
\hline SIS & 6 & 0.580 & 0.889 & $.824(\mathrm{c})$ & 0.816 & 0.831 \\
\hline ZMB & 10 & 0.410 & 0.996 & $.988(c)$ & 0.986 & 0.990 \\
\hline SSS & 8 & 0.553 & 0.920 & $.865(c)$ & 0.858 & 0.872 \\
\hline FMB & 9 & 0.427 & 0.993 & $.979(\mathrm{c})$ & 0.977 & 0.982 \\
\hline NAS & 8 & 0.707 & 0.699 & $.613(\mathrm{c})$ & 0.603 & 0.622 \\
\hline EKB & 9 & 0.607 & 0.855 & $.785(c)$ & 0.777 & 0.793 \\
\hline DKS & 8 & 0.491 & 0.970 & $.939(\mathrm{c})$ & 0.934 & 0.943 \\
\hline IML & 7 & 0.477 & 0.977 & $.948(\mathrm{c})$ & 0.943 & 0.952 \\
\hline XML-Z & 7 & 0.927 & 0.356 & $.274(\mathrm{c})$ & 0.265 & 0.283 \\
\hline MLS & 6 & 0.618 & 0.839 & $.762(\mathrm{c})$ & 0.754 & 0.770 \\
\hline WMH & 11 & 0.629 & 0.823 & $.757(\mathrm{c})$ & 0.749 & 0.766 \\
\hline GLS & 9 & 1.000 & 0.270 & $.207(c)$ & 0.199 & 0.215 \\
\hline STB & 6 & 0.532 & 0.940 & $.890(\mathrm{c})$ & 0.883 & 0.896 \\
\hline FRC & 8 & 0.422 & 0.994 & $.984(\mathrm{c})$ & 0.981 & 0.986 \\
\hline FRS & 8 & 0.598 & 0.867 & $.796(\mathrm{c})$ & 0.789 & 0.804 \\
\hline FRF & 8 & 0.444 & 0.989 & $.973(\mathrm{c})$ & 0.970 & 0.976 \\
\hline PAC & 9 & 0.576 & 0.895 & $.836(\mathrm{c})$ & 0.829 & 0.843 \\
\hline PAS & 9 & 0.324 & 1.000 & $.999(\mathrm{c})$ & 0.999 & 1.000 \\
\hline PAF & 9 & 0.388 & 0.998 & $.992(\mathrm{c})$ & 0.990 & 0.994 \\
\hline OCC & 7 & 0.766 & 0.600 & $.501(\mathrm{c})$ & 0.491 & 0.511 \\
\hline ocs & 7 & 0.609 & 0.853 & $.777(\mathrm{c})$ & 0.769 & 0.785 \\
\hline OCF & 7 & 0.495 & 0.967 & $.931(\mathrm{c})$ & 0.926 & 0.936 \\
\hline FOL & 6 & 0.474 & 0.978 & $.948(\mathrm{c})$ & 0.944 & 0.952 \\
\hline FOB & 8 & 0.631 & 0.820 & $.741(\mathrm{c})$ & 0.732 & 0.749 \\
\hline UFBR & 8 & 0.904 & 0.387 & $.307(\mathrm{c})$ & 0.298 & 0.316 \\
\hline GNI & 12 & 0.494 & 0.967 & $.939(\mathrm{c})$ & 0.934 & 0.944 \\
\hline HML & 14 & 0.456 & 0.985 & $.968(\mathrm{c})$ & 0.965 & 0.971 \\
\hline TML & 14 & 0.673 & 0.755 & $.691(\mathrm{c})$ & 0.682 & 0.700 \\
\hline GOG & 10 & 0.664 & 0.771 & $.700(\mathrm{c})$ & 0.691 & 0.709 \\
\hline CDL & 6 & 0.458 & 0.985 & .959 (c) & 0.955 & 0.963 \\
\hline WRB & 11 & 0.567 & 0.904 & $.850(\mathrm{c})$ & 0.843 & 0.857 \\
\hline $\mathrm{XRH}$ & 10 & 0.602 & 0.862 & $.800(\mathrm{c})$ & 0.793 & 0.808 \\
\hline MXML & 10 & 0.878 & 0.423 & $.356(c)$ & 0.347 & 0.365 \\
\hline MAN & 11 & 0.524 & 0.946 & .907 (c) & 0.902 & 0.913 \\
\hline
\end{tabular}

(c) Based on 10000 sampled tables 
Appendix 11 continued. Kolmogorov-Smirnov test of normality for craniometric variables with Monte Carlo approximations

\begin{tabular}{|c|c|c|c|c|c|c|}
\hline \multicolumn{7}{|c|}{ Post-2000 BP Females } \\
\hline \multirow[b]{3}{*}{ Variable } & \multirow[b]{3}{*}{$\mathrm{N}$} & \multirow[b]{3}{*}{ K-S Z } & \multirow[b]{3}{*}{$\mathrm{p}$} & \multirow[b]{3}{*}{$\mathrm{p}$} & \multicolumn{2}{|c|}{ Monte Carlo } \\
\hline & & & & & \multicolumn{2}{|c|}{ 95\% C.I. } \\
\hline & & & & & Lower Bound & Upper Bound \\
\hline GOL & 16 & 1.142 & 0.147 & $.112(\mathrm{c})$ & 0.106 & 0.118 \\
\hline NOL & 16 & 0.724 & 0.671 & $.606(\mathrm{c})$ & 0.597 & 0.616 \\
\hline BNL & 15 & 0.596 & 0.870 & $.810(\mathrm{c})$ & 0.802 & 0.817 \\
\hline $\mathrm{BBH}$ & 15 & 0.609 & 0.853 & $.790(c)$ & 0.782 & 0.798 \\
\hline XCB & 16 & 0.589 & 0.879 & $.828(c)$ & 0.821 & 0.836 \\
\hline XFB & 14 & 0.419 & 0.995 & $.984(\mathrm{c})$ & 0.982 & 0.987 \\
\hline WFB & 16 & 0.659 & 0.778 & $.717(\mathrm{c})$ & 0.708 & 0.726 \\
\hline ZYB & 13 & 0.755 & 0.618 & $.549(\mathrm{c})$ & 0.539 & 0.559 \\
\hline AUB & 15 & 0.934 & 0.348 & $.288(\mathrm{c})$ & 0.279 & 0.297 \\
\hline ASB & 17 & 0.653 & 0.787 & $.737(\mathrm{c})$ & 0.728 & 0.746 \\
\hline BPL & 15 & 1.003 & 0.267 & $.213(c)$ & 0.205 & 0.221 \\
\hline $\mathrm{NPH}$ & 17 & 0.323 & 1.000 & 1.000 (c) & 0.999 & 1.000 \\
\hline $\mathrm{NLH}$ & 17 & 0.632 & 0.819 & $.770(\mathrm{c})$ & 0.762 & 0.779 \\
\hline JUB & 15 & 0.780 & 0.577 & $.504(\mathrm{c})$ & 0.495 & 0.514 \\
\hline NLB & 17 & 1.068 & 0.204 & $.170(c)$ & 0.163 & 0.178 \\
\hline MAB & 16 & 0.512 & 0.956 & $.925(\mathrm{c})$ & 0.920 & 0.930 \\
\hline MAL & 16 & 0.865 & 0.442 & $.386(\mathrm{c})$ & 0.377 & 0.396 \\
\hline $\mathrm{MDH}$ & 17 & 0.646 & 0.799 & .749 (c) & 0.741 & 0.758 \\
\hline $\mathrm{OBH}$ & 16 & 0.811 & 0.526 & $.469(\mathrm{c})$ & 0.459 & 0.479 \\
\hline OBB & 15 & 0.584 & 0.885 & $.831(\mathrm{c})$ & 0.824 & 0.838 \\
\hline DKB & 16 & 0.817 & 0.517 & $.460(\mathrm{c})$ & 0.451 & 0.470 \\
\hline NDS & 14 & 1.079 & 0.194 & $.153(c)$ & 0.146 & 0.160 \\
\hline WNB & 14 & 0.921 & 0.364 & $.307(c)$ & 0.298 & 0.316 \\
\hline SIS & 14 & 0.847 & 0.470 & $.406(c)$ & 0.397 & 0.416 \\
\hline ZMB & 14 & 0.585 & 0.883 & $.833(\mathrm{c})$ & 0.825 & 0.840 \\
\hline SSS & 14 & 0.585 & 0.884 & $.833(\mathrm{c})$ & 0.826 & 0.840 \\
\hline FMB & 16 & 0.579 & 0.890 & $.841(\mathrm{c})$ & 0.834 & 0.848 \\
\hline NAS & 16 & 0.822 & 0.508 & $.451(\mathrm{c})$ & 0.441 & 0.460 \\
\hline EKB & 15 & 0.645 & 0.799 & $.730(c)$ & 0.721 & 0.739 \\
\hline DKS & 14 & 0.731 & 0.658 & $.592(\mathrm{c})$ & 0.582 & 0.602 \\
\hline $\mathrm{IML}$ & 13 & 0.405 & 0.997 & $.992(\mathrm{c})$ & 0.990 & 0.994 \\
\hline XML-Z & 13 & 0.513 & 0.955 & $.922(\mathrm{c})$ & 0.917 & 0.928 \\
\hline MLS & 13 & 0.929 & 0.354 & $.301(c)$ & 0.292 & 0.310 \\
\hline WMH & 16 & 0.631 & 0.820 & $.761(\mathrm{c})$ & 0.752 & 0.769 \\
\hline GLS & 16 & 0.959 & 0.316 & $.264(\mathrm{c})$ & 0.255 & 0.272 \\
\hline STB & 15 & 0.699 & 0.714 & $.637(\mathrm{c})$ & 0.628 & 0.647 \\
\hline FRC & 16 & 0.750 & 0.627 & $.564(\mathrm{c})$ & 0.554 & 0.573 \\
\hline FRS & 16 & 0.693 & 0.723 & $.658(\mathrm{c})$ & 0.649 & 0.668 \\
\hline FRF & 16 & 1.110 & 0.170 & $.132(\mathrm{c})$ & 0.125 & 0.138 \\
\hline PAC & 17 & 0.527 & 0.944 & $.910(\mathrm{c})$ & 0.904 & 0.916 \\
\hline PAS & 17 & 0.537 & 0.936 & .899 (c) & 0.893 & 0.905 \\
\hline PAF & 17 & 0.730 & 0.662 & $.605(\mathrm{c})$ & 0.596 & 0.615 \\
\hline OCC & 15 & 0.701 & 0.710 & $.634(\mathrm{c})$ & 0.625 & 0.644 \\
\hline OCS & 15 & 0.885 & 0.414 & $.348(c)$ & 0.339 & 0.358 \\
\hline OCF & 15 & 0.632 & 0.819 & $.752(c)$ & 0.743 & 0.760 \\
\hline FOL & 14 & 0.753 & 0.622 & $.553(\mathrm{c})$ & 0.543 & 0.563 \\
\hline FOB & 16 & 0.676 & 0.750 & $.688(\mathrm{c})$ & 0.679 & 0.697 \\
\hline UFBR & 15 & 1.075 & 0.198 & $.154(\mathrm{c})$ & 0.147 & 0.161 \\
\hline GNI & 16 & 0.712 & 0.691 & $.627(\mathrm{c})$ & 0.617 & 0.636 \\
\hline HML & 16 & 0.970 & 0.303 & $.253(\mathrm{c})$ & 0.244 & 0.262 \\
\hline TML & 16 & 0.550 & 0.923 & $.882(c)$ & 0.875 & 0.888 \\
\hline GOG & 15 & 0.530 & 0.941 & $.899(\mathrm{c})$ & 0.893 & 0.905 \\
\hline CDL & 14 & 0.536 & 0.936 & $.898(c)$ & 0.892 & 0.904 \\
\hline WRB & 15 & 0.547 & 0.926 & $.881(\mathrm{c})$ & 0.874 & 0.887 \\
\hline $\mathrm{XRH}$ & 15 & 0.407 & 0.996 & $.987(\mathrm{c})$ & 0.984 & 0.989 \\
\hline MXML & 14 & 0.750 & 0.627 & $.558(\mathrm{c})$ & 0.548 & 0.568 \\
\hline MAN & 15 & 0.625 & 0.830 & $.762(\mathrm{c})$ & 0.753 & 0.770 \\
\hline
\end{tabular}

c. Based on 10000 sampled tables 
Appendix 11 continued. Kolmogorov-Smirnov test of normality for craniometric variables with Monte Carlo approximations

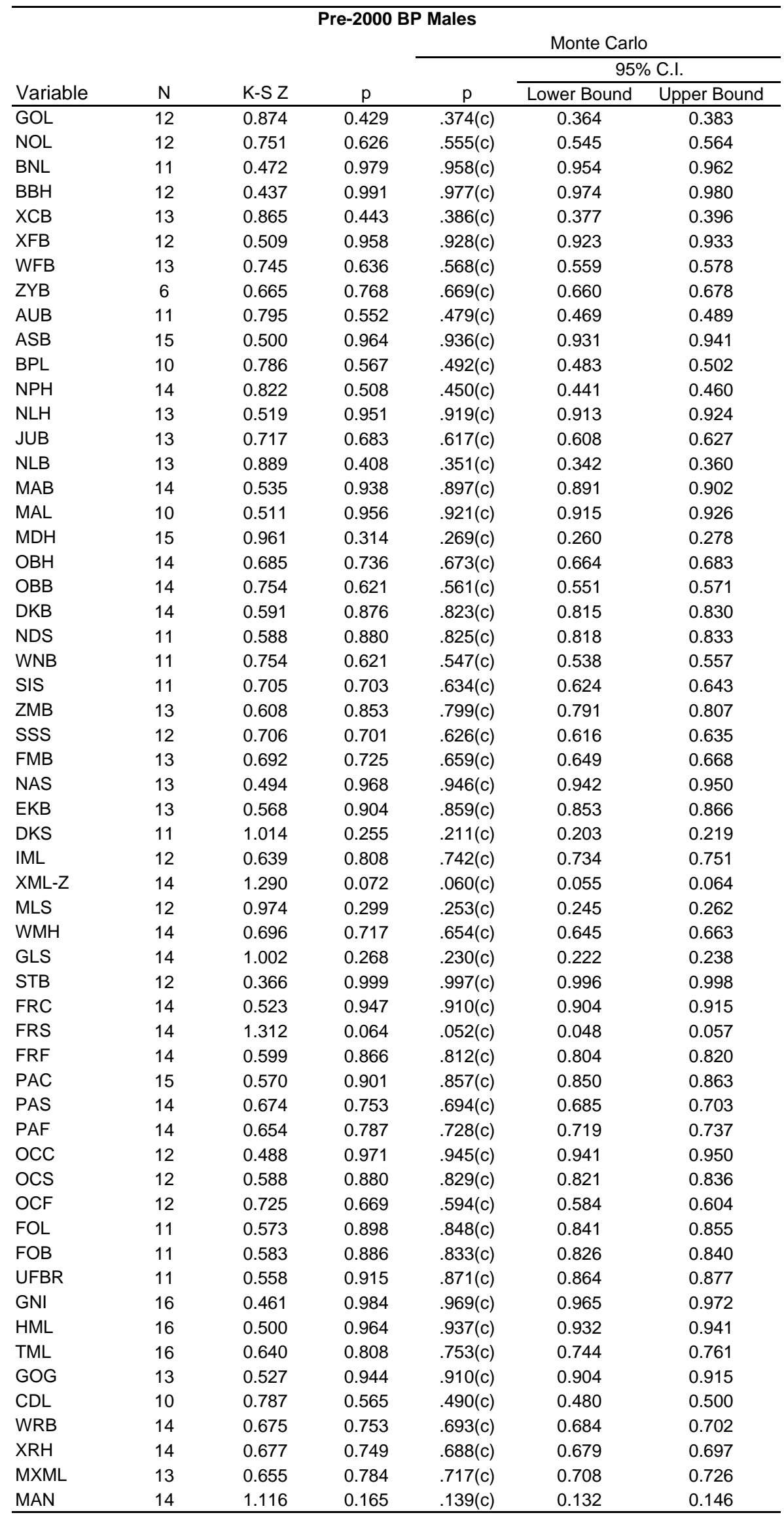

c. Based on 10000 sampled tables 
Appendix 11 continued. Kolmogorov-Smirnov test of normality for craniometric variables with Monte Carlo approximations

\begin{tabular}{|c|c|c|c|c|c|c|}
\hline \multicolumn{7}{|c|}{ Post-2000 BP Males } \\
\hline \multirow[b]{3}{*}{ Variable } & \multirow[b]{3}{*}{$\mathrm{N}$} & \multirow[b]{3}{*}{ K-S Z } & \multirow[b]{3}{*}{$p$} & \multicolumn{3}{|c|}{ Monte Carlo } \\
\hline & & & & & \multicolumn{2}{|c|}{ 95\% C.I. } \\
\hline & & & & $\mathrm{p}$ & Lower Bound & Upper Bound \\
\hline$\overline{\mathrm{GOL}}$ & 9 & 0.601 & 0.863 & $.799(\mathrm{c})$ & 0.791 & 0.807 \\
\hline NOL & 9 & 0.465 & 0.982 & $.959(\mathrm{c})$ & 0.956 & 0.963 \\
\hline BNL & 9 & 0.929 & 0.354 & $.285(c)$ & 0.276 & 0.294 \\
\hline $\mathrm{BBH}$ & 9 & 0.485 & 0.973 & $.944(c)$ & 0.939 & 0.948 \\
\hline XCB & 8 & 0.672 & 0.757 & $.676(c)$ & 0.666 & 0.685 \\
\hline XFB & 8 & 0.745 & 0.636 & $.552(\mathrm{c})$ & 0.542 & 0.562 \\
\hline WFB & 9 & 0.456 & 0.985 & $.965(c)$ & 0.961 & 0.968 \\
\hline ZYB & 8 & 0.783 & 0.572 & $.487(c)$ & 0.477 & 0.496 \\
\hline AUB & 9 & 0.537 & 0.935 & $.890(\mathrm{c})$ & 0.884 & 0.896 \\
\hline ASB & 9 & 0.691 & 0.726 & $.647(c)$ & 0.637 & 0.656 \\
\hline BPL & 9 & 0.503 & 0.962 & $.927(\mathrm{c})$ & 0.922 & 0.932 \\
\hline NPH & 9 & 0.678 & 0.748 & $.670(c)$ & 0.661 & 0.679 \\
\hline NLH & 9 & 0.571 & 0.901 & $.841(\mathrm{c})$ & 0.834 & 0.848 \\
\hline JUB & 9 & 0.788 & 0.563 & $.483(\mathrm{c})$ & 0.473 & 0.493 \\
\hline NLB & 9 & 0.489 & 0.971 & .940 (c) & 0.935 & 0.945 \\
\hline MAB & 9 & 0.725 & 0.669 & $.590(c)$ & 0.581 & 0.600 \\
\hline MAL & 9 & 0.783 & 0.571 & $.492(c)$ & 0.482 & 0.501 \\
\hline $\mathrm{MDH}$ & 9 & 0.530 & 0.942 & .899 (c) & 0.893 & 0.905 \\
\hline $\mathrm{OBH}$ & 9 & 0.815 & 0.520 & $.442(c)$ & 0.432 & 0.452 \\
\hline OBB & 9 & 0.592 & 0.875 & $.813(c)$ & 0.805 & 0.820 \\
\hline DKB & 9 & 0.627 & 0.827 & $.755(c)$ & 0.747 & 0.764 \\
\hline NDS & 9 & 0.862 & 0.448 & $.373(c)$ & 0.364 & 0.383 \\
\hline WNB & 9 & 0.636 & 0.813 & .740 (c) & 0.731 & 0.748 \\
\hline SIS & 9 & 0.539 & 0.934 & $.888(c)$ & 0.882 & 0.894 \\
\hline ZMB & 8 & 0.480 & 0.975 & .945 (c) & 0.940 & 0.949 \\
\hline SSS & 8 & 0.490 & 0.970 & $.935(c)$ & 0.930 & 0.939 \\
\hline FMB & 9 & 0.342 & 1.000 & .999 (c) & 0.998 & 0.999 \\
\hline NAS & 9 & 0.833 & 0.491 & $.415(c)$ & 0.405 & 0.425 \\
\hline EKB & 9 & 0.638 & 0.811 & .737 (c) & 0.728 & 0.746 \\
\hline DKS & 9 & 0.527 & 0.944 & $.903(c)$ & 0.897 & 0.908 \\
\hline $\mathrm{IML}$ & 9 & 0.640 & 0.807 & $.733(c)$ & 0.724 & 0.741 \\
\hline XML-Z & 9 & 0.500 & 0.964 & .930 (c) & 0.925 & 0.935 \\
\hline MLS & 9 & 0.667 & 0.764 & $.686(c)$ & 0.677 & 0.695 \\
\hline WMH & 9 & 0.384 & 0.998 & $.992(c)$ & 0.990 & 0.994 \\
\hline GLS & 9 & 0.941 & 0.339 & $.272(c)$ & 0.263 & 0.281 \\
\hline STB & 8 & 0.571 & 0.900 & .839 (c) & 0.832 & 0.847 \\
\hline FRC & 9 & 0.403 & 0.997 & $.988(c)$ & 0.986 & 0.990 \\
\hline FRS & 9 & 0.630 & 0.823 & $.751(\mathrm{c})$ & 0.742 & 0.759 \\
\hline FRF & 9 & 0.990 & 0.281 & $.222(c)$ & 0.213 & 0.230 \\
\hline PAC & 9 & 0.673 & 0.756 & $.678(c)$ & 0.669 & 0.687 \\
\hline PAS & 9 & 0.507 & 0.960 & $.923(c)$ & 0.917 & 0.928 \\
\hline PAF & 9 & 0.437 & 0.991 & $.976(c)$ & 0.973 & 0.979 \\
\hline OCC & 9 & 0.400 & 0.997 & .989 (c) & 0.987 & 0.991 \\
\hline ocs & 9 & 0.843 & 0.476 & .399 (c) & 0.389 & 0.409 \\
\hline OCF & 9 & 1.063 & 0.208 & $.162(c)$ & 0.154 & 0.169 \\
\hline FOL & 9 & 0.354 & 1.000 & $.997(c)$ & 0.996 & 0.998 \\
\hline FOB & 9 & 0.664 & 0.770 & $.693(c)$ & 0.684 & 0.702 \\
\hline UFBR & 9 & 0.633 & 0.817 & $.744(\mathrm{c})$ & 0.736 & 0.753 \\
\hline GNI & 7 & 0.422 & 0.994 & $.977(\mathrm{c})$ & 0.974 & 0.980 \\
\hline HML & 7 & 0.490 & 0.970 & $.936(c)$ & 0.932 & 0.941 \\
\hline TML & 7 & 0.423 & 0.994 & .977 (c) & 0.974 & 0.980 \\
\hline GOG & 6 & 0.728 & 0.665 & $.567(c)$ & 0.557 & 0.576 \\
\hline CDL & 6 & 0.527 & 0.944 & $.894(\mathrm{c})$ & 0.888 & 0.900 \\
\hline WRB & 7 & 0.462 & 0.983 & $.956(c)$ & 0.952 & 0.960 \\
\hline $\mathrm{XRH}$ & 6 & 0.661 & 0.775 & $.689(\mathrm{c})$ & 0.680 & 0.698 \\
\hline MXML & 6 & 0.573 & 0.898 & $.830(c)$ & 0.823 & 0.837 \\
\hline MAN & 6 & 0.446 & 0.989 & $.966(\mathrm{c})$ & 0.962 & 0.969 \\
\hline
\end{tabular}

c. Based on 10000 sampled tables 
Appendix 12. Kolmogorov-Smirnov test of normality for maximum craniometric variables with Monte Carlo approximations

\begin{tabular}{|c|c|c|c|c|c|c|}
\hline \multirow[b]{4}{*}{ Variables } & \multirow[b]{4}{*}{$\mathrm{N}$} & \multicolumn{3}{|c|}{ Pre-2000 BP Females } & \\
\hline & & & \multirow[b]{3}{*}{$\mathrm{p}$} & \multirow[b]{3}{*}{$\mathrm{p}$} & Monte Carlc & \\
\hline & & & & & \multicolumn{2}{|c|}{ 95\% C.I. } \\
\hline & & $\mathrm{K}-\mathrm{S} \mathrm{Z}$ & & & Lower Bound & Upper Bound \\
\hline UI1MBL & 7 & 0.569 & 0.903 & $.845(c)$ & 0.838 & 0.852 \\
\hline UI1MMD & 3 & 0.652 & 0.788 & $.660(c)$ & 0.651 & 0.669 \\
\hline UI1MCRA & 3 & 0.598 & 0.867 & $.747(\mathrm{c})$ & 0.739 & 0.756 \\
\hline UI2MBL & 7 & 0.578 & 0.892 & $.830(c)$ & 0.822 & 0.837 \\
\hline UI2MMD & 2 & 0.368 & 0.999 & $.999(c)$ & 0.999 & 1.000 \\
\hline UI2MCRA & 2 & 0.368 & 0.999 & $.999(c)$ & 0.999 & 1.000 \\
\hline UCMBL & 8 & 0.458 & 0.985 & $.964(c)$ & 0.960 & 0.967 \\
\hline UCMMD & 3 & 0.369 & 0.999 & $.995(c)$ & 0.994 & 0.996 \\
\hline UCMCRA & 3 & 0.340 & 1.000 & $.998(c)$ & 0.998 & 0.999 \\
\hline UP1MBL & 7 & 0.601 & 0.863 & $.792(c)$ & 0.784 & 0.800 \\
\hline UP1MMD & 4 & 0.540 & 0.932 & $.856(c)$ & 0.850 & 0.863 \\
\hline UP1MCRA & 4 & 0.488 & 0.971 & $.921(c)$ & 0.916 & 0.926 \\
\hline UP2MBL & 8 & 0.402 & 0.997 & $.989(c)$ & 0.987 & 0.991 \\
\hline UP2MMD & 4 & 0.577 & 0.893 & $.806(c)$ & 0.798 & 0.813 \\
\hline UP2MCRA & 4 & 0.300 & 1.000 & $1.000(\mathrm{c})$ & 1.000 & 1.000 \\
\hline UM1MBL & 5 & 0.852 & 0.462 & $.367(\mathrm{c})$ & 0.357 & 0.376 \\
\hline UM1MMD & 2 & 0.368 & 0.999 & $.999(c)$ & 0.999 & 1.000 \\
\hline UM1MCRA & 2 & 0.368 & 0.999 & $.999(c)$ & 0.999 & 1.000 \\
\hline UM2MBL & 6 & 0.893 & 0.403 & $.319(c)$ & 0.310 & 0.328 \\
\hline UM2MMD & 3 & 0.422 & 0.994 & $.978(c)$ & 0.975 & 0.981 \\
\hline UM2MCRA & 3 & 0.517 & 0.952 & $.890(c)$ & 0.884 & 0.896 \\
\hline UM3MBL & 4 & 0.680 & 0.744 & $.635(c)$ & 0.626 & 0.645 \\
\hline LI1MBL & 6 & 0.586 & 0.882 & $.808(c)$ & 0.800 & 0.816 \\
\hline LI1MMD & 2 & 0.368 & 0.999 & $.999(c)$ & 0.999 & 1.000 \\
\hline LI1MCRA & 2 & 0.368 & 0.999 & $.999(c)$ & 0.999 & 1.000 \\
\hline LI2MBL & 8 & 0.395 & 0.998 & $.991(c)$ & 0.989 & 0.993 \\
\hline LI2MMD & 2 & 0.368 & 0.999 & $.999(c)$ & 0.999 & 1.000 \\
\hline LI2MCRA & 2 & 0.368 & 0.999 & $.999(c)$ & 0.999 & 1.000 \\
\hline LCMBL & 9 & 0.397 & 0.998 & $.990(c)$ & 0.988 & 0.992 \\
\hline LCMMD & 4 & 0.663 & 0.772 & $.664(c)$ & 0.655 & 0.674 \\
\hline LCMCRA & 4 & 0.503 & 0.962 & $.902(c)$ & 0.896 & 0.908 \\
\hline LP1MBL & 11 & 0.477 & 0.977 & $.954(c)$ & 0.950 & 0.958 \\
\hline LP1MMD & 6 & 0.614 & 0.845 & $.765(c)$ & 0.757 & 0.773 \\
\hline LP1MCRA & 6 & 0.471 & 0.980 & $.947(c)$ & 0.942 & 0.951 \\
\hline LP2MBL & 10 & 0.570 & 0.901 & $.840(c)$ & 0.833 & 0.847 \\
\hline LP2MMD & 6 & 0.435 & 0.992 & $.973(c)$ & 0.970 & 0.976 \\
\hline LP2MCRA & 6 & 0.565 & 0.907 & $.840(c)$ & 0.833 & 0.847 \\
\hline LM1MBL & 6 & 0.514 & 0.954 & $.905(c)$ & 0.899 & 0.911 \\
\hline LM1MMD & 3 & 0.466 & 0.982 & $.949(c)$ & 0.944 & 0.953 \\
\hline LM1MCRA & 3 & 0.362 & 0.999 & $.996(c)$ & 0.995 & 0.997 \\
\hline LM2MBL & 9 & 0.735 & 0.653 & $.573(c)$ & 0.563 & 0.582 \\
\hline LM2MMD & 4 & 0.374 & 0.999 & $.993(c)$ & 0.992 & 0.995 \\
\hline LM2MCRA & 4 & 0.637 & 0.812 & $.711(\mathrm{c})$ & 0.702 & 0.720 \\
\hline LM3MBL & 8 & 0.731 & 0.660 & $.565(c)$ & 0.555 & 0.574 \\
\hline LM3MMD & 2 & 0.368 & 0.999 & $.999(c)$ & 0.999 & 1.000 \\
\hline LM3MCRA & 2 & 0.368 & 0.999 & $.999(c)$ & 0.999 & 1.000 \\
\hline
\end{tabular}

c. Based on 10000 sampled tables 
Appendix 12 continued. Kolmogorov-Smirnov test of normality for maximum craniometric variables with Monte Carlo approximations

\begin{tabular}{|c|c|c|c|c|c|c|}
\hline \multicolumn{7}{|c|}{ Post-2000 BP Females } \\
\hline \multirow[b]{3}{*}{ Variables } & \multirow[b]{3}{*}{$\mathrm{N}$} & \multirow[b]{3}{*}{ K-S Z } & \multirow[b]{3}{*}{$\mathrm{p}$} & \multirow[b]{3}{*}{$\mathrm{p}$} & \multicolumn{2}{|l|}{ Monte Carlo } \\
\hline & & & & & \multicolumn{2}{|c|}{ 95\% C.I. } \\
\hline & & & & & Lower Bound & Upper Bound \\
\hline UI1MBL & 2 & 0.368 & 0.999 & $.999(\mathrm{c})$ & 0.999 & 1.000 \\
\hline UI2MBL & 3 & 0.448 & 0.988 & $.963(c)$ & 0.959 & 0.967 \\
\hline UCMBL & 7 & 0.565 & 0.907 & $.850(\mathrm{c})$ & 0.842 & 0.857 \\
\hline UP1MBL & 8 & 0.726 & 0.668 & $.576(\mathrm{c})$ & 0.567 & 0.586 \\
\hline UP1MMD & 3 & 0.500 & 0.964 & $.910(\mathrm{c})$ & 0.904 & 0.915 \\
\hline UP1MCRA & 3 & 0.531 & 0.941 & $.863(\mathrm{c})$ & 0.856 & 0.870 \\
\hline UP2MBL & 7 & 0.817 & 0.516 & $.427(\mathrm{c})$ & 0.417 & 0.437 \\
\hline UP2MMD & 2 & 0.368 & 0.999 & $.999(\mathrm{c})$ & 0.999 & 1.000 \\
\hline UP2MCRA & 2 & 0.368 & 0.999 & $.999(c)$ & 0.999 & 1.000 \\
\hline UM1MBL & 8 & 0.648 & 0.795 & $.711(\mathrm{c})$ & 0.702 & 0.719 \\
\hline UM1MMD & 2 & 0.368 & 0.999 & $.999(\mathrm{c})$ & 0.999 & 1.000 \\
\hline UM1MCRA & 2 & 0.368 & 0.999 & $.999(\mathrm{c})$ & 0.999 & 1.000 \\
\hline UM2MBL & 9 & 0.566 & 0.906 & $.849(\mathrm{c})$ & 0.842 & 0.856 \\
\hline UM2MMD & 3 & 0.426 & 0.993 & $.975(c)$ & 0.972 & 0.978 \\
\hline UM2MCRA & 3 & 0.380 & 0.999 & $.992(\mathrm{c})$ & 0.990 & 0.994 \\
\hline UM3MBL & 10 & 0.945 & 0.333 & $.263(\mathrm{c})$ & 0.254 & 0.272 \\
\hline UM3MMD & 5 & 0.386 & 0.998 & $.993(\mathrm{c})$ & 0.991 & 0.994 \\
\hline UM3MCRA & 5 & 0.612 & 0.847 & $.768(\mathrm{c})$ & 0.760 & 0.776 \\
\hline LI1MBL & 4 & 0.505 & 0.961 & $.897(\mathrm{c})$ & 0.891 & 0.903 \\
\hline LI2MBL & 5 & 0.500 & 0.964 & $.917(\mathrm{c})$ & 0.911 & 0.922 \\
\hline LCMBL & 8 & 0.625 & 0.830 & $.751(\mathrm{c})$ & 0.742 & 0.759 \\
\hline LP1MBL & 8 & 0.503 & 0.962 & $.920(c)$ & 0.914 & 0.925 \\
\hline LP1MMD & 2 & 0.368 & 0.999 & $.999(c)$ & 0.999 & 1.000 \\
\hline LP1MCRA & 2 & 0.368 & 0.999 & $.999(\mathrm{c})$ & 0.999 & 1.000 \\
\hline LP2MBL & 5 & 0.535 & 0.937 & $.877(\mathrm{c})$ & 0.870 & 0.883 \\
\hline LM1MBL & 5 & 0.477 & 0.977 & $.938(\mathrm{c})$ & 0.933 & 0.942 \\
\hline LM1MMD & 2 & 0.368 & 0.999 & $.999(\mathrm{c})$ & 0.999 & 1.000 \\
\hline LM1MCRA & 2 & 0.368 & 0.999 & $.999(\mathrm{c})$ & 0.999 & 1.000 \\
\hline LM2MBL & 7 & 0.701 & 0.710 & $.622(\mathrm{c})$ & 0.612 & 0.631 \\
\hline LM2MMD & 3 & 0.469 & 0.980 & $.945(c)$ & 0.940 & 0.949 \\
\hline LM2MCRA & 3 & 0.653 & 0.788 & $.648(c)$ & 0.639 & 0.657 \\
\hline LM3MBL & 9 & 0.552 & 0.920 & $.868(\mathrm{c})$ & 0.861 & 0.875 \\
\hline LM3MMD & 3 & 0.359 & 1.000 & $.996(c)$ & 0.995 & 0.998 \\
\hline LM3MCRA & 3 & 0.613 & 0.847 & $.712(\mathrm{c})$ & 0.703 & 0.721 \\
\hline
\end{tabular}

c. Based on 10000 sampled tables 
Appendix 12 continued. Kolmogorov-Smirnov test of normality for maximum craniometric variables with Monte Carlo approximations

\begin{tabular}{|c|c|c|c|c|c|c|}
\hline \multirow[b]{4}{*}{ Variables } & \multirow[b]{4}{*}{$\mathrm{N}$} & \multicolumn{3}{|c|}{ Pre-2000 BP Males } & & \\
\hline & & & \multirow[b]{3}{*}{$p$} & \multicolumn{3}{|c|}{ Monte Carlo } \\
\hline & & & & \multirow[b]{2}{*}{$p$} & \multicolumn{2}{|c|}{ 95\% C.I. } \\
\hline & & $\mathrm{K}-\mathrm{S} Z \mathrm{Z}$ & & & Lower Bound & Upper Bound \\
\hline UI1MBL & 4 & 0.615 & 0.843 & $.750(c)$ & 0.742 & 0.759 \\
\hline UI1MMD & 3 & 0.615 & 0.843 & $.708(c)$ & 0.699 & 0.716 \\
\hline UI1MCRA & 3 & 0.630 & 0.823 & $.684(c)$ & 0.675 & 0.693 \\
\hline UI2MBL & 4 & 0.411 & 0.996 & $.985(c)$ & 0.983 & 0.987 \\
\hline UI2MMD & 3 & 0.597 & 0.869 & $.738(c)$ & 0.730 & 0.747 \\
\hline UI2MCRA & 3 & 0.547 & 0.926 & $.836(c)$ & 0.829 & 0.844 \\
\hline UCMBL & 6 & 0.551 & 0.921 & $.859(c)$ & 0.852 & 0.866 \\
\hline UCMMD & 3 & 0.308 & 1.000 & $1.000(\mathrm{c})$ & 1.000 & 1.000 \\
\hline UCMCRA & 3 & 0.341 & 1.000 & $.999(c)$ & 0.998 & 0.999 \\
\hline UP1MBL & 6 & 0.551 & 0.922 & $.859(c)$ & 0.853 & 0.866 \\
\hline UP1MMD & 3 & 0.306 & 1.000 & $1.000(\mathrm{c})$ & 1.000 & 1.000 \\
\hline UP1MCRA & 3 & 0.598 & 0.866 & $.734(c)$ & 0.726 & 0.743 \\
\hline UP2MBL & 6 & 0.611 & 0.850 & $.766(c)$ & 0.758 & 0.774 \\
\hline UP2MMD & 3 & 0.636 & 0.814 & $.674(c)$ & 0.665 & 0.683 \\
\hline UP2MCRA & 3 & 0.432 & 0.992 & $.973(c)$ & 0.970 & 0.976 \\
\hline UM1MBL & 5 & 0.724 & 0.671 & $.574(c)$ & 0.564 & 0.584 \\
\hline UM1MMD & 2 & 0.368 & 0.999 & $.999(c)$ & 0.998 & 0.999 \\
\hline UM1MCRA & 2 & 0.368 & 0.999 & $.999(c)$ & 0.998 & 0.999 \\
\hline UM2MBL & 7 & 0.438 & 0.991 & $.973(c)$ & 0.970 & 0.977 \\
\hline UM2MMD & 2 & 0.368 & 0.999 & $.999(c)$ & 0.998 & 0.999 \\
\hline UM2MCRA & 2 & 0.368 & 0.999 & $.999(c)$ & 0.998 & 0.999 \\
\hline UM3MBL & 5 & 0.821 & 0.511 & $.411(\mathrm{c})$ & 0.401 & 0.420 \\
\hline UM3MMD & 2 & 0.368 & 0.999 & $.999(c)$ & 0.998 & 0.999 \\
\hline UM3MCRA & 2 & 0.368 & 0.999 & $.999(c)$ & 0.998 & 0.999 \\
\hline LI1MBL & 5 & 0.514 & 0.954 & $.905(c)$ & 0.899 & 0.911 \\
\hline LI1MMD & 3 & 0.456 & 0.985 & $.957(c)$ & 0.953 & 0.961 \\
\hline LI1MCRA & 3 & 0.658 & 0.780 & $.642(c)$ & 0.633 & 0.652 \\
\hline LI2MBL & 5 & 0.437 & 0.991 & $.971(\mathrm{c})$ & 0.968 & 0.974 \\
\hline LI2MMD & 2 & 0.368 & 0.999 & $.999(c)$ & 0.998 & 0.999 \\
\hline LI2MCRA & 2 & 0.368 & 0.999 & $.999(c)$ & 0.998 & 0.999 \\
\hline LCMBL & 6 & 0.428 & 0.993 & $.975(c)$ & 0.971 & 0.978 \\
\hline LCMMD & 2 & 0.368 & 0.999 & $.999(c)$ & 0.998 & 0.999 \\
\hline LCMCRA & 2 & 0.368 & 0.999 & $.999(c)$ & 0.998 & 0.999 \\
\hline LP1MBL & 8 & 0.401 & 0.997 & $.990(c)$ & 0.988 & 0.992 \\
\hline LP1MMD & 4 & 0.553 & 0.919 & $.840(c)$ & 0.833 & 0.847 \\
\hline LP1MCRA & 4 & 0.630 & 0.822 & $.727(\mathrm{c})$ & 0.718 & 0.735 \\
\hline LP2MBL & 7 & 0.643 & 0.803 & $.720(c)$ & 0.712 & 0.729 \\
\hline LP2MMD & 3 & 0.438 & 0.991 & $.970(c)$ & 0.966 & 0.973 \\
\hline LP2MCRA & 3 & 0.623 & 0.832 & $.694(c)$ & 0.685 & 0.703 \\
\hline LM1MBL & 5 & 0.425 & 0.994 & $.978(c)$ & 0.976 & 0.981 \\
\hline LM1MMD & 3 & 0.412 & 0.996 & $.981(c)$ & 0.979 & 0.984 \\
\hline LM1MCRA & 3 & 0.642 & 0.804 & $.665(c)$ & 0.656 & 0.674 \\
\hline LM2MBL & 6 & 0.722 & 0.674 & $.573(c)$ & 0.563 & 0.582 \\
\hline LM2MMD & 3 & 0.466 & 0.982 & $.949(c)$ & 0.944 & 0.953 \\
\hline LM2MCRA & 3 & 0.514 & 0.954 & $.893(c)$ & 0.887 & 0.899 \\
\hline LM3MBL & 5 & 0.432 & 0.992 & $.974(c)$ & 0.971 & 0.977 \\
\hline
\end{tabular}

c. Based on 10000 sampled tables 
Appendix 12 continued. Kolmogorov-Smirnov test of normality for maximum craniometric variables with Monte Carlo approximations

\begin{tabular}{|c|c|c|c|c|c|c|}
\hline \multicolumn{7}{|c|}{ Post-2000 BP Males } \\
\hline \multirow[b]{3}{*}{ Variables } & \multirow[b]{3}{*}{$\mathrm{N}$} & \multirow[b]{3}{*}{ K-S Z } & \multirow[b]{3}{*}{$\mathrm{p}$} & \multicolumn{3}{|c|}{ Monte Carlo } \\
\hline & & & & & \multicolumn{2}{|c|}{ 95\% C.I. } \\
\hline & & & & $\mathrm{p}$ & Lower Bound & Upper Bound \\
\hline UI1MBL & 3 & 0.423 & 0.994 & $.976(\mathrm{c})$ & 0.973 & 0.979 \\
\hline UI2MBL & 5 & 0.344 & 1.000 & $.998(\mathrm{c})$ & 0.997 & 0.999 \\
\hline UI2MMD & 2 & 0.368 & 0.999 & $.999(\mathrm{c})$ & 0.999 & 1.000 \\
\hline UI2MCRA & 2 & 0.368 & 0.999 & $.999(\mathrm{c})$ & 0.999 & 1.000 \\
\hline UCMBL & 6 & 1.056 & 0.215 & $.163(\mathrm{c})$ & 0.156 & 0.170 \\
\hline UP1MBL & 4 & 0.465 & 0.982 & $.951(\mathrm{c})$ & 0.946 & 0.955 \\
\hline UP1MMD & 2 & 0.368 & 0.999 & $.999(\mathrm{c})$ & 0.999 & 1.000 \\
\hline UP1MCRA & 2 & 0.368 & 0.999 & $.999(\mathrm{c})$ & 0.999 & 1.000 \\
\hline UP2MBL & 5 & 0.512 & 0.956 & $.904(\mathrm{c})$ & 0.898 & 0.910 \\
\hline UP2MMD & 2 & 0.368 & 0.999 & $.999(\mathrm{c})$ & 0.999 & 1.000 \\
\hline UP2MCRA & 2 & 0.368 & 0.999 & $.999(\mathrm{c})$ & 0.999 & 1.000 \\
\hline UM1MBL & 5 & 0.473 & 0.978 & $.943(\mathrm{c})$ & 0.938 & 0.947 \\
\hline UM1MMD & 3 & 0.502 & 0.963 & $.911(\mathrm{c})$ & 0.905 & 0.916 \\
\hline UM1MCRA & 3 & 0.489 & 0.971 & $.927(\mathrm{c})$ & 0.921 & 0.932 \\
\hline UM2MBL & 6 & 0.476 & 0.977 & $.945(\mathrm{c})$ & 0.941 & 0.950 \\
\hline UM2MMD & 3 & 0.619 & 0.839 & $.715(\mathrm{c})$ & 0.706 & 0.724 \\
\hline UM2MCRA & 3 & 0.609 & 0.852 & $.729(\mathrm{c})$ & 0.720 & 0.738 \\
\hline UM3MBL & 3 & 0.347 & 1.000 & $.998(\mathrm{c})$ & 0.997 & 0.999 \\
\hline UM3MMD & 3 & 0.521 & 0.949 & $.884(\mathrm{c})$ & 0.878 & 0.890 \\
\hline UM3MCRA & 3 & 0.428 & 0.993 & $.974(\mathrm{c})$ & 0.970 & 0.977 \\
\hline LIIMBL & 4 & 0.400 & 0.997 & $.989(c)$ & 0.987 & 0.991 \\
\hline LI2MBL & 6 & 0.681 & 0.743 & $.653(\mathrm{c})$ & 0.644 & 0.663 \\
\hline LCMBL & 5 & 0.565 & 0.907 & $.834(\mathrm{c})$ & 0.827 & 0.841 \\
\hline LCMMD & 2 & 0.368 & 0.999 & $.999(\mathrm{c})$ & 0.999 & 1.000 \\
\hline LCMCRA & 2 & 0.368 & 0.999 & $.999(\mathrm{c})$ & 0.999 & 1.000 \\
\hline LP1MBL & 5 & 0.597 & 0.868 & $.787(\mathrm{c})$ & 0.779 & 0.795 \\
\hline LP1MMD & 2 & 0.368 & 0.999 & $.999(\mathrm{c})$ & 0.999 & 1.000 \\
\hline LP1MCRA & 2 & 0.368 & 0.999 & $.999(\mathrm{c})$ & 0.999 & 1.000 \\
\hline LP2MBL & 5 & 0.742 & 0.640 & $.538(\mathrm{c})$ & 0.528 & 0.548 \\
\hline LP2MMD & 2 & 0.368 & 0.999 & $.999(\mathrm{c})$ & 0.999 & 1.000 \\
\hline LP2MCRA & 2 & 0.368 & 0.999 & $.999(\mathrm{c})$ & 0.999 & 1.000 \\
\hline LM1MBL & 3 & 0.667 & 0.766 & $.636(\mathrm{c})$ & 0.626 & 0.645 \\
\hline LM2MBL & 6 & 0.556 & 0.916 & $.854(\mathrm{c})$ & 0.847 & 0.860 \\
\hline LM2MMD & 3 & 0.306 & 1.000 & $1.000(\mathrm{c})$ & 1.000 & 1.000 \\
\hline LM2MCRA & 3 & 0.325 & 1.000 & $1.000(\mathrm{c})$ & 0.999 & 1.000 \\
\hline LM3MBL & 3 & 0.490 & 0.970 & $.926(c)$ & 0.921 & 0.931 \\
\hline LM3MMD & 2 & 0.368 & 0.999 & $.999(\mathrm{c})$ & 0.999 & 1.000 \\
\hline LM3MCRA & 2 & 0.368 & 0.999 & $.999(\mathrm{c})$ & 0.999 & 1.000 \\
\hline
\end{tabular}

c. Based on 10000 sampled tables 
Appendix 13. Kolmogorov-Smirnov test of normality for cervical odontometric variables with Monte Carlo approximations

\begin{tabular}{|c|c|c|c|c|c|c|}
\hline \multirow[b]{4}{*}{ Variable } & \multicolumn{5}{|c|}{ Pre-2000 BP Females } & \\
\hline & \multirow[b]{3}{*}{$\mathrm{N}$} & \multirow[b]{3}{*}{ K-S Z } & \multirow[b]{3}{*}{$\mathrm{p}$} & \multicolumn{3}{|c|}{ Monte Carlo } \\
\hline & & & & & \multicolumn{2}{|c|}{$95 \%$ C.I. } \\
\hline & & & & $\mathrm{p}$ & Lower Bound & Upper Bound \\
\hline UI1CCRA & 7 & 0.544 & 0.929 & $.878(\mathrm{c})$ & 0.872 & 0.885 \\
\hline UI2CBL & 6 & 0.448 & 0.988 & $.964(\mathrm{c})$ & 0.960 & 0.967 \\
\hline UI2CMD & 7 & 0.842 & 0.478 & $.397(\mathrm{c})$ & 0.387 & 0.407 \\
\hline UI2CCRA & 6 & 0.688 & 0.731 & $.646(c)$ & 0.636 & 0.655 \\
\hline UCCBL & 9 & 0.358 & 1.000 & $.996(\mathrm{c})$ & 0.995 & 0.998 \\
\hline UCCMD & 9 & 0.440 & 0.990 & $.974(\mathrm{c})$ & 0.970 & 0.977 \\
\hline UCCCRA & 8 & 0.530 & 0.941 & $.892(\mathrm{c})$ & 0.886 & 0.898 \\
\hline UP1CBL & 9 & 0.441 & 0.990 & $.973(c)$ & 0.970 & 0.976 \\
\hline UP1CMD & 9 & 0.702 & 0.707 & $.631(\mathrm{c})$ & 0.622 & 0.640 \\
\hline UP1CCRA & 9 & 0.392 & 0.998 & $.991(c)$ & 0.989 & 0.993 \\
\hline UP2CBL & 10 & 0.766 & 0.601 & $.521(\mathrm{c})$ & 0.511 & 0.530 \\
\hline UP2CMD & 9 & 0.577 & 0.893 & $.838(\mathrm{c})$ & 0.831 & 0.845 \\
\hline UP2CCRA & 9 & 0.480 & 0.975 & $.947(c)$ & 0.942 & 0.951 \\
\hline UM1CBL & 9 & 0.429 & 0.993 & $.978(c)$ & 0.975 & 0.981 \\
\hline UM1CMD & 7 & 0.493 & 0.968 & $.934(\mathrm{c})$ & 0.929 & 0.939 \\
\hline UMICCRA & 7 & 0.518 & 0.951 & $.908(c)$ & 0.902 & 0.914 \\
\hline UM2CBL & 12 & 0.603 & 0.860 & $.801(\mathrm{c})$ & 0.794 & 0.809 \\
\hline UM2CMD & 10 & 0.509 & 0.958 & $.921(\mathrm{c})$ & 0.915 & 0.926 \\
\hline UM2CCRA & 9 & 0.552 & 0.921 & $.873(c)$ & 0.866 & 0.879 \\
\hline UM3CBL & 5 & 0.686 & 0.735 & $.634(c)$ & 0.625 & 0.644 \\
\hline UM3CMD & 5 & 0.737 & 0.650 & $.545(\mathrm{c})$ & 0.535 & 0.555 \\
\hline UM3CCRA & 5 & 0.482 & 0.974 & $.934(c)$ & 0.929 & 0.939 \\
\hline LI1CBL & 7 & 0.465 & 0.982 & $.957(c)$ & 0.953 & 0.960 \\
\hline LI1CMD & 5 & 0.605 & 0.858 & $.775(c)$ & 0.766 & 0.783 \\
\hline LI1CCRA & 5 & 0.436 & 0.991 & $.970(c)$ & 0.966 & 0.973 \\
\hline LI2CBL & 9 & 0.541 & 0.932 & $.886(c)$ & 0.880 & 0.893 \\
\hline LI2CMD & 9 & 0.523 & 0.947 & $.908(c)$ & 0.902 & 0.913 \\
\hline LI2CCRA & 9 & 0.470 & 0.980 & $.952(\mathrm{c})$ & 0.948 & 0.956 \\
\hline LCCBL & 9 & 0.780 & 0.576 & $.494(c)$ & 0.485 & 0.504 \\
\hline LCCMD & 9 & 0.362 & 0.999 & $.996(c)$ & 0.994 & 0.997 \\
\hline LCCCRA & 9 & 0.441 & 0.990 & $.972(\mathrm{c})$ & 0.969 & 0.976 \\
\hline LP1CBL & 13 & 0.410 & 0.996 & $.989(c)$ & 0.986 & 0.991 \\
\hline LP1CMD & 12 & 0.649 & 0.794 & $.728(c)$ & 0.719 & 0.737 \\
\hline LP1CCRA & 12 & 0.787 & 0.565 & $.505(c)$ & 0.495 & 0.515 \\
\hline LP2CBL & 11 & 0.614 & 0.846 & $.788(c)$ & 0.780 & 0.796 \\
\hline LP2CMD & 11 & 0.670 & 0.760 & $.693(c)$ & 0.684 & 0.702 \\
\hline LP2CCRA & 11 & 0.447 & 0.988 & $.974(\mathrm{c})$ & 0.971 & 0.977 \\
\hline LM1CBL & 10 & 0.465 & 0.982 & $.960(c)$ & 0.956 & 0.964 \\
\hline LM1CMD & 9 & 0.827 & 0.500 & $.423(c)$ & 0.414 & 0.433 \\
\hline LM1CCRA & 8 & 0.563 & 0.909 & $.851(\mathrm{c})$ & 0.844 & 0.858 \\
\hline LM2CBL & 12 & 0.619 & 0.839 & $.776(c)$ & 0.768 & 0.784 \\
\hline LM2CMD & 10 & 0.363 & 0.999 & $.996(\mathrm{c})$ & 0.995 & 0.997 \\
\hline LM2CCRA & 9 & 0.786 & 0.567 & $.485(c)$ & 0.475 & 0.495 \\
\hline LM3CBL & 9 & 0.590 & 0.878 & $.818(c)$ & 0.810 & 0.825 \\
\hline LM3CMD & 3 & 0.409 & 0.996 & $.984(\mathrm{c})$ & 0.981 & 0.986 \\
\hline LM3CCRA & 2 & 0.368 & 0.999 & $.999(\mathrm{c})$ & 0.999 & 1.000 \\
\hline
\end{tabular}

c. Based on 10000 sampled tables 
Appendix 13 continued. Kolmogorov-Smirnov test of normality for cervical odontometric variables with Monte Carlo approximations

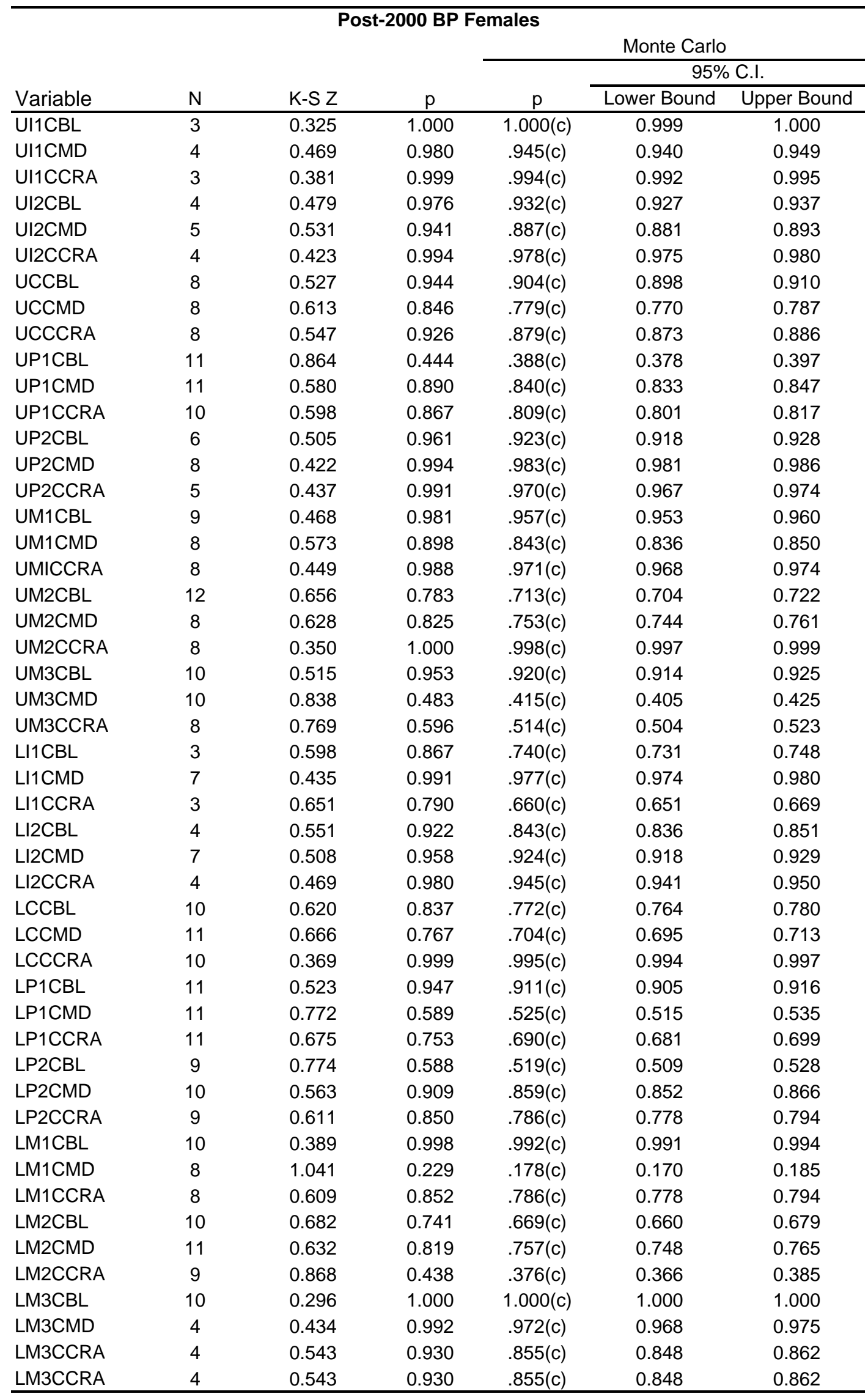

c. Based on 10000 sampled tables 
Appendix 13 continued. Kolmogorov-Smirnov test of normality for cervical odontometric variables with Monte Carlo approximations

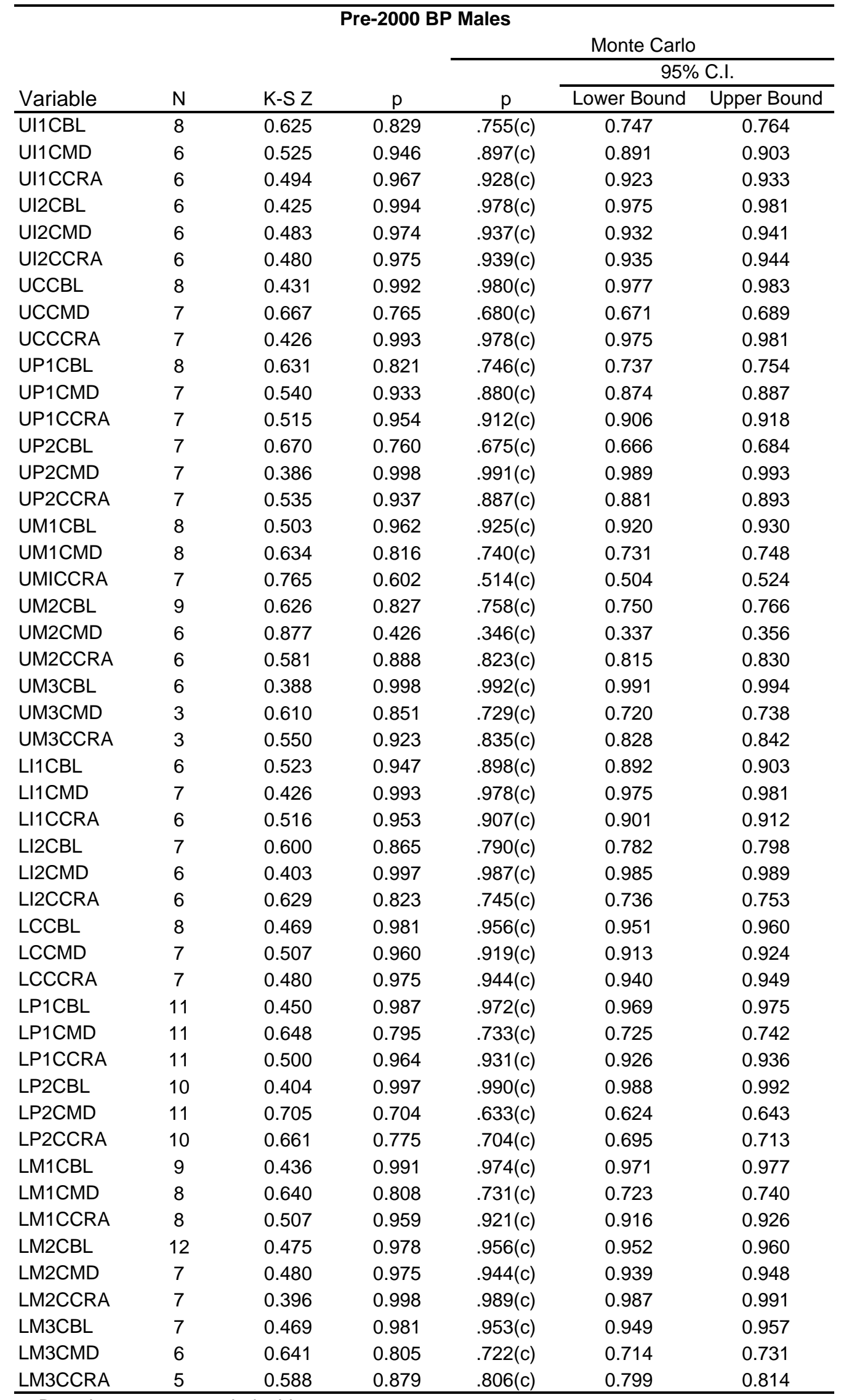

c. Based on 10000 sampled tables 
Appendix 13 continued. Kolmogorov-Smirnov test of normality for cervical odontometric variables with Monte Carlo approximations

\begin{tabular}{|c|c|c|c|c|c|c|}
\hline \multirow[b]{4}{*}{ Variable } & \multirow[b]{4}{*}{$\mathrm{N}$} & \multicolumn{3}{|c|}{ Post-2000 BP Males } & \\
\hline & & & \multirow[b]{3}{*}{$\mathrm{p}$} & \multirow[b]{3}{*}{$\mathrm{p}$} & Monte Carlc & \\
\hline & & & & & \multicolumn{2}{|c|}{ 95\% C.I. } \\
\hline & & K-S Z & & & Lower Bound & Upper Bound \\
\hline UI1CBL & 4 & 0.544 & 0.929 & $.851(\mathrm{c})$ & 0.844 & 0.857 \\
\hline UI1CMD & 4 & 0.559 & 0.913 & $.830(\mathrm{c})$ & 0.823 & 0.837 \\
\hline UI1CCRA & 3 & 0.382 & 0.999 & $.994(\mathrm{c})$ & 0.993 & 0.996 \\
\hline UI2CBL & 7 & 0.552 & 0.921 & $.860(\mathrm{c})$ & 0.853 & 0.867 \\
\hline UI2CMD & 6 & 0.330 & 1.000 & $.999(c)$ & 0.998 & 1.000 \\
\hline UI2CCRA & 6 & 0.443 & 0.989 & $.967(\mathrm{c})$ & 0.964 & 0.971 \\
\hline UCCBL & 8 & 0.645 & 0.800 & $.725(c)$ & 0.716 & 0.734 \\
\hline UCCMD & 8 & 0.480 & 0.976 & $.949(\mathrm{c})$ & 0.944 & 0.953 \\
\hline UCCCRA & 8 & 0.462 & 0.983 & $.960(\mathrm{c})$ & 0.956 & 0.964 \\
\hline UP1CBL & 8 & 0.619 & 0.838 & $.765(\mathrm{c})$ & 0.757 & 0.774 \\
\hline UP1CMD & 9 & 0.715 & 0.687 & $.610(\mathrm{c})$ & 0.601 & 0.620 \\
\hline UP1CCRA & 8 & 0.492 & 0.969 & $.938(\mathrm{c})$ & 0.933 & 0.942 \\
\hline UP2CBL & 6 & 0.711 & 0.692 & $.599(\mathrm{c})$ & 0.589 & 0.608 \\
\hline UP2CMD & 8 & 0.484 & 0.974 & $.946(c)$ & 0.942 & 0.951 \\
\hline UP2CCRA & 6 & 0.671 & 0.760 & $.670(\mathrm{c})$ & 0.661 & 0.680 \\
\hline UM1CBL & 8 & 0.619 & 0.838 & $.766(c)$ & 0.758 & 0.775 \\
\hline UM1CMD & 7 & 0.524 & 0.947 & $.901(\mathrm{c})$ & 0.895 & 0.907 \\
\hline UMICCRA & 6 & 0.448 & 0.988 & $.965(c)$ & 0.961 & 0.968 \\
\hline UM2CBL & 7 & 0.520 & 0.950 & $.905(c)$ & 0.900 & 0.911 \\
\hline UM2CMD & 7 & 0.470 & 0.980 & $.953(c)$ & 0.948 & 0.957 \\
\hline UM2CCRA & 6 & 0.682 & 0.742 & $.651(\mathrm{c})$ & 0.642 & 0.661 \\
\hline UM3CBL & 6 & 0.648 & 0.795 & $.713(c)$ & 0.704 & 0.721 \\
\hline UM3CMD & 6 & 0.713 & 0.689 & $.594(c)$ & 0.584 & 0.604 \\
\hline UM3CCRA & 6 & 0.395 & 0.998 & $.988(c)$ & 0.986 & 0.990 \\
\hline LI1CBL & 5 & 0.655 & 0.784 & $.694(c)$ & 0.684 & 0.703 \\
\hline LI1CMD & 5 & 0.468 & 0.981 & $.947(\mathrm{c})$ & 0.942 & 0.951 \\
\hline LI1CCRA & 5 & 0.564 & 0.908 & $.839(\mathrm{c})$ & 0.832 & 0.846 \\
\hline LI2CBL & 6 & 0.731 & 0.659 & $.559(\mathrm{c})$ & 0.549 & 0.569 \\
\hline LI2CMD & 7 & 0.534 & 0.938 & $.886(c)$ & 0.879 & 0.892 \\
\hline LI2CCRA & 6 & 0.439 & 0.991 & $.970(\mathrm{c})$ & 0.966 & 0.973 \\
\hline LCCBL & 6 & 0.428 & 0.993 & $.976(c)$ & 0.972 & 0.979 \\
\hline LCCMD & 6 & 0.630 & 0.822 & $.746(c)$ & 0.737 & 0.754 \\
\hline LCCCRA & 6 & 0.578 & 0.892 & $.829(c)$ & 0.822 & 0.836 \\
\hline LP1CBL & 7 & 0.579 & 0.891 & $.821(\mathrm{c})$ & 0.814 & 0.829 \\
\hline LP1CMD & 7 & 0.655 & 0.785 & $.697(\mathrm{c})$ & 0.688 & 0.706 \\
\hline LP1CCRA & 7 & 0.401 & 0.997 & $.990(\mathrm{c})$ & 0.988 & 0.992 \\
\hline LP2CBL & 7 & 0.587 & 0.880 & $.810(\mathrm{c})$ & 0.803 & 0.818 \\
\hline LP2CMD & 7 & 0.399 & 0.997 & $.990(\mathrm{c})$ & 0.988 & 0.992 \\
\hline LP2CCRA & 7 & 0.527 & 0.944 & $.896(c)$ & 0.890 & 0.902 \\
\hline LM1CBL & 5 & 0.588 & 0.880 & $.804(c)$ & 0.796 & 0.812 \\
\hline LM1CMD & 6 & 0.547 & 0.925 & $.873(c)$ & 0.866 & 0.879 \\
\hline LM1CCRA & 4 & 0.464 & 0.983 & $.951(\mathrm{c})$ & 0.946 & 0.955 \\
\hline LM2CBL & 8 & 0.473 & 0.979 & $.953(c)$ & 0.949 & 0.957 \\
\hline LM2CMD & 8 & 0.532 & 0.940 & $.893(c)$ & 0.887 & 0.899 \\
\hline LM2CCRA & 8 & 0.587 & 0.881 & $.817(\mathrm{c})$ & 0.809 & 0.824 \\
\hline LM3CBL & 3 & 0.304 & 1.000 & $1.000(\mathrm{c})$ & 1.000 & 1.000 \\
\hline LM3CMD & 2 & 0.368 & 0.999 & $.999(\mathrm{c})$ & 0.998 & 0.999 \\
\hline
\end{tabular}

c. Based on 10000 sampled tables 
Appendix 14. Kolmogorov-Smirnov test of normality for postcranial metric variables with Monte Carlo approximations

\begin{tabular}{|c|c|c|c|c|c|c|}
\hline \multicolumn{7}{|c|}{ Pre-2000 BP Females } \\
\hline \multirow[b]{3}{*}{ Variables } & \multirow[b]{3}{*}{$\mathrm{N}$} & \multirow[b]{3}{*}{$\mathrm{K}-\mathrm{S} Z \mathrm{Z}$} & \multirow[b]{3}{*}{$\mathrm{p}$} & \multirow[b]{3}{*}{$\mathrm{p}$} & \multicolumn{2}{|c|}{ Monte Carlo } \\
\hline & & & & & \multicolumn{2}{|c|}{$95 \%$ C.I. } \\
\hline & & & & & Lower Bound & Upper Bound \\
\hline$\overline{C L A V X L}$ & 14 & 0.701 & 0.709 & $.643(\mathrm{c})$ & 0.633 & 0.652 \\
\hline CLAVAPD & 13 & 1.021 & 0.248 & $.200(c)$ & 0.192 & 0.208 \\
\hline CLAVSID & 14 & 1.047 & 0.223 & $.181(\mathrm{c})$ & 0.173 & 0.188 \\
\hline CLAVRBSTINDX & 14 & 0.806 & 0.534 & $.465(c)$ & 0.455 & 0.474 \\
\hline SCAPXHT & 5 & 0.485 & 0.973 & $.930(\mathrm{c})$ & 0.924 & 0.935 \\
\hline SCAPXBR & 7 & 0.529 & 0.942 & $.894(c)$ & 0.888 & 0.900 \\
\hline SCAPSPIL & 8 & 0.497 & 0.966 & $.936(c)$ & 0.931 & 0.941 \\
\hline SCAPGCBR & 14 & 0.928 & 0.355 & $.297(\mathrm{c})$ & 0.288 & 0.306 \\
\hline SCAPGCHT & 14 & 1.135 & 0.152 & $.117(\mathrm{c})$ & 0.111 & 0.123 \\
\hline SCAPINDX & 4 & 0.526 & 0.945 & $.875(\mathrm{c})$ & 0.869 & 0.881 \\
\hline HUMXL & 13 & 0.527 & 0.944 & $.910(\mathrm{c})$ & 0.905 & 0.916 \\
\hline HUMMIDXD & 14 & 0.924 & 0.360 & $.302(\mathrm{c})$ & 0.293 & 0.311 \\
\hline HUMMIDND & 14 & 0.722 & 0.675 & $.610(\mathrm{c})$ & 0.600 & 0.619 \\
\hline HUMMIDSHFTINDX & 14 & 0.642 & 0.805 & $.742(\mathrm{c})$ & 0.733 & 0.750 \\
\hline HUMHEADD & 14 & 0.991 & 0.280 & $.232(\mathrm{c})$ & 0.223 & 0.240 \\
\hline HUMEPBR & 14 & 0.712 & 0.692 & $.625(\mathrm{c})$ & 0.615 & 0.634 \\
\hline HUMRBSTINDX & 13 & 0.424 & 0.994 & $.983(\mathrm{c})$ & 0.981 & 0.986 \\
\hline CLAHUMINDX & 12 & 0.773 & 0.588 & $.519(\mathrm{c})$ & 0.509 & 0.529 \\
\hline RADXL & 11 & 0.405 & 0.997 & $.991(\mathrm{c})$ & 0.989 & 0.992 \\
\hline RADHEADD & 12 & 0.953 & 0.324 & $.269(\mathrm{c})$ & 0.260 & 0.277 \\
\hline RADAPD & 14 & 0.995 & 0.275 & $.227(\mathrm{c})$ & 0.219 & 0.235 \\
\hline RADMLD & 14 & 0.609 & 0.853 & $.794(c)$ & 0.786 & 0.802 \\
\hline RADSHFTINDX & 14 & 1.353 & 0.051 & $.035(\mathrm{c})$ & 0.031 & 0.039 \\
\hline RADHUMINDX & 10 & 0.780 & 0.577 & $.501(\mathrm{c})$ & 0.491 & 0.510 \\
\hline ULNAXL & 12 & 0.620 & 0.836 & $.768(c)$ & 0.760 & 0.777 \\
\hline ULNAPHYL & 10 & 0.698 & 0.714 & $.638(\mathrm{c})$ & 0.629 & 0.648 \\
\hline ULNAXOBR & 12 & 0.612 & 0.847 & $.784(\mathrm{c})$ & 0.776 & 0.792 \\
\hline ULNANOBR & 13 & 0.759 & 0.613 & $.543(\mathrm{c})$ & 0.533 & 0.553 \\
\hline ULNAAPD & 12 & 1.074 & 0.199 & $.161(\mathrm{c})$ & 0.154 & 0.168 \\
\hline ULNAMLD & 12 & 1.544 & 0.017 & $.013(\mathrm{c})$ & 0.011 & 0.015 \\
\hline ULNASHFTINDX & 12 & 1.000 & 0.270 & $.222(\mathrm{c})$ & 0.214 & 0.230 \\
\hline SKTRNKHT & 3 & 0.436 & 0.991 & $.969(\mathrm{c})$ & 0.965 & 0.972 \\
\hline SACRANTH & 7 & 0.511 & 0.956 & $.915(\mathrm{c})$ & 0.909 & 0.920 \\
\hline SACRANTB & 9 & 0.504 & 0.962 & $.931(\mathrm{c})$ & 0.926 & 0.936 \\
\hline SACRS1BR & 9 & 0.464 & 0.982 & $.964(\mathrm{c})$ & 0.960 & 0.967 \\
\hline SACRINDX & 6 & 0.480 & 0.975 & $.944(\mathrm{c})$ & 0.940 & 0.949 \\
\hline INNOHT & 7 & 0.841 & 0.479 & $.390(\mathrm{c})$ & 0.380 & 0.399 \\
\hline ILIACBR & 9 & 0.693 & 0.722 & $.641(\mathrm{c})$ & 0.631 & 0.650 \\
\hline PUBISL & 6 & 0.528 & 0.943 & $.895(c)$ & 0.889 & 0.901 \\
\hline ISCHIUML & 8 & 0.726 & 0.668 & $.583(c)$ & 0.573 & 0.593 \\
\hline BIILIACBR & 6 & 0.630 & 0.822 & $.741(\mathrm{c})$ & 0.733 & 0.750 \\
\hline ISCPUBINDX & 5 & 0.502 & 0.963 & $.913(\mathrm{c})$ & 0.908 & 0.919 \\
\hline FEMXL & 13 & 0.575 & 0.895 & $.847(\mathrm{c})$ & 0.839 & 0.854 \\
\hline FEMBL & 13 & 0.508 & 0.959 & $.932(\mathrm{c})$ & 0.927 & 0.937 \\
\hline FEMSTAPD & 15 & 1.125 & 0.159 & $.125(c)$ & 0.119 & 0.132 \\
\hline FEMSTMLD & 15 & 0.993 & 0.278 & $.232(\mathrm{c})$ & 0.224 & 0.240 \\
\hline PLATYMINDX & 15 & 1.084 & 0.191 & $.153(\mathrm{c})$ & 0.146 & 0.160 \\
\hline FEMMIDADL & 15 & 0.849 & 0.467 & $.407(\mathrm{c})$ & 0.398 & 0.417 \\
\hline FEMMIDMD & 15 & 1.113 & 0.168 & $.135(\mathrm{c})$ & 0.128 & 0.141 \\
\hline FEMMIDSHFTINDX & 15 & 0.607 & 0.855 & $.803(\mathrm{c})$ & 0.795 & 0.811 \\
\hline FEMVHEDD & 12 & 0.542 & 0.930 & $.885(c)$ & 0.879 & 0.891 \\
\hline FEMHHEDD & 14 & 0.591 & 0.876 & $.823(\mathrm{c})$ & 0.816 & 0.831 \\
\hline FEMLCAPD & 14 & 0.515 & 0.953 & $.919(\mathrm{c})$ & 0.914 & 0.924 \\
\hline FEMMCAPD & 12 & 0.774 & 0.586 & $.517(\mathrm{c})$ & 0.507 & 0.527 \\
\hline FEMEPICB & 12 & 0.574 & 0.897 & $.841(\mathrm{c})$ & 0.834 & 0.848 \\
\hline FEMMIDCR & 15 & 0.563 & 0.909 & $.864(\mathrm{c})$ & 0.857 & 0.870 \\
\hline FEMRBSTINDX & 13 & 0.530 & 0.941 & $.906(c)$ & 0.901 & 0.912 \\
\hline TIBXL & 11 & 0.379 & 0.999 & $.996(c)$ & 0.994 & 0.997 \\
\hline TIBPEPBR & 11 & 0.792 & 0.557 & $.484(c)$ & 0.475 & 0.494 \\
\hline TIBDEPBR & 13 & 0.837 & 0.486 & $.424(c)$ & 0.415 & 0.434 \\
\hline TIBAPNFD & 13 & 0.918 & 0.368 & $.314(\mathrm{c})$ & 0.304 & 0.323 \\
\hline TIBMLNFD & 13 & 0.764 & 0.603 & $.535(\mathrm{c})$ & 0.525 & 0.544 \\
\hline PLATYCNINDX & 13 & 0.637 & 0.812 & $.753(c)$ & 0.745 & 0.762 \\
\hline TIBNUTFC & 13 & 0.549 & 0.924 & $.885(\mathrm{c})$ & 0.878 & 0.891 \\
\hline TIBFEMINDX & 10 & 0.576 & 0.895 & $.838(\mathrm{c})$ & 0.831 & 0.845 \\
\hline FIBXL & 6 & 0.735 & 0.652 & $.553(\mathrm{c})$ & 0.543 & 0.562 \\
\hline FIBMSXD & 9 & 0.807 & 0.532 & $.449(c)$ & 0.439 & 0.459 \\
\hline CALCXLN & 10 & 0.517 & 0.952 & $.912(\mathrm{c})$ & 0.906 & 0.917 \\
\hline CALCMBR & 10 & 0.971 & 0.302 & $.244(c)$ & 0.235 & 0.252 \\
\hline MT1MXHT & 9 & 0.563 & 0.909 & $.857(\mathrm{c})$ & 0.850 & 0.864 \\
\hline MT1AP & 9 & 0.977 & 0.296 & $.231(\mathrm{c})$ & 0.223 & 0.240 \\
\hline MT1ML & 8 & 0.811 & 0.526 & $.441(\mathrm{c})$ & 0.431 & 0.451 \\
\hline
\end{tabular}

c. Based on 10000 sampled tables 


\begin{tabular}{|c|c|c|c|c|c|c|}
\hline \multicolumn{7}{|c|}{ Post-2000 BP Females } \\
\hline \multirow[b]{3}{*}{ Variables } & \multirow[b]{3}{*}{$\mathrm{N}$} & \multirow[b]{3}{*}{ K-S Z } & \multirow[b]{3}{*}{$\mathrm{p}$} & \multirow[b]{3}{*}{$\mathrm{p}$} & \multicolumn{2}{|l|}{ Monte Carlo } \\
\hline & & & & & \multicolumn{2}{|c|}{ 95\% C.I. } \\
\hline & & & & & Lower Bound & Upper Bound \\
\hline CLAVXL & 12 & 0.654 & 0.785 & $.724(\mathrm{c})$ & 0.715 & 0.732 \\
\hline CLAVAPD & 13 & 0.666 & 0.766 & $.705(c)$ & 0.696 & 0.714 \\
\hline CLAVSID & 13 & 1.028 & 0.241 & $.200(c)$ & 0.192 & 0.208 \\
\hline CLAVRBSTINDX & 12 & 0.428 & 0.993 & $.985(c)$ & 0.982 & 0.987 \\
\hline SCAPXHT & 9 & 0.481 & 0.975 & $.952(\mathrm{c})$ & 0.947 & 0.956 \\
\hline SCAPXBR & 13 & 0.716 & 0.685 & $.626(\mathrm{c})$ & 0.617 & 0.636 \\
\hline SCAPSPIL & 12 & 0.558 & 0.914 & $.867(c)$ & 0.860 & 0.873 \\
\hline SCAPGCBR & 14 & 0.593 & 0.873 & $.825(c)$ & 0.817 & 0.832 \\
\hline SCAPGCHT & 14 & 0.534 & 0.938 & $.903(c)$ & 0.897 & 0.908 \\
\hline SCAPINDX & 9 & 0.716 & 0.685 & $.608(c)$ & 0.599 & 0.618 \\
\hline HUMXL & 14 & 0.487 & 0.972 & $.950(c)$ & 0.946 & 0.954 \\
\hline HUMMIDXD & 15 & 0.720 & 0.678 & $.616(\mathrm{c})$ & 0.606 & 0.625 \\
\hline HUMMIDND & 15 & 0.885 & 0.414 & $.364(c)$ & 0.354 & 0.373 \\
\hline HUMMIDSHFTINDX & 15 & 0.952 & 0.325 & $.280(\mathrm{c})$ & 0.271 & 0.289 \\
\hline HUMHEADD & 14 & 0.896 & 0.399 & $.346(c)$ & 0.336 & 0.355 \\
\hline HUMEPBR & 14 & 0.965 & 0.310 & $.264(c)$ & 0.255 & 0.272 \\
\hline HUMRBSTINDX & 14 & 0.387 & 0.998 & $.995(c)$ & 0.994 & 0.996 \\
\hline CLAHUMINDX & 12 & 0.515 & 0.954 & $.920(c)$ & 0.914 & 0.925 \\
\hline RADXL & 12 & 0.463 & 0.983 & $.965(c)$ & 0.962 & 0.969 \\
\hline RADHEADD & 13 & 0.849 & 0.467 & $.412(\mathrm{c})$ & 0.402 & 0.421 \\
\hline RADAPD & 14 & 1.155 & 0.139 & $.110(c)$ & 0.104 & 0.116 \\
\hline RADMLD & 14 & 0.707 & 0.699 & $.644(c)$ & 0.634 & 0.653 \\
\hline RADSHFTINDX & 14 & 1.029 & 0.240 & $.198(c)$ & 0.190 & 0.205 \\
\hline RADHUMINDX & 11 & 0.454 & 0.986 & .969 (c) & 0.965 & 0.972 \\
\hline ULNAXL & 12 & 0.578 & 0.892 & $.839(c)$ & 0.832 & 0.846 \\
\hline ULNAPHYL & 12 & 0.570 & 0.902 & $.850(c)$ & 0.843 & 0.857 \\
\hline ULNAXOBR & 13 & 0.800 & 0.545 & $.488(c)$ & 0.479 & 0.498 \\
\hline ULNANOBR & 13 & 1.001 & 0.269 & $.226(c)$ & 0.218 & 0.235 \\
\hline ULNAAPD & 14 & 0.717 & 0.683 & $.626(c)$ & 0.616 & 0.635 \\
\hline ULNAMLD & 14 & 0.889 & 0.407 & $.354(c)$ & 0.344 & 0.363 \\
\hline ULNASHFTINDX & 14 & 0.444 & 0.989 & $.977(\mathrm{c})$ & 0.974 & 0.980 \\
\hline SKTRNKHT & 8 & 0.465 & 0.982 & $.961(\mathrm{c})$ & 0.957 & 0.965 \\
\hline SACRANTH & 12 & 0.951 & 0.326 & $.273(c)$ & 0.264 & 0.282 \\
\hline SACRANTB & 13 & 0.715 & 0.686 & $.628(c)$ & 0.619 & 0.637 \\
\hline SACRS1BR & 14 & 0.603 & 0.861 & $.811(c)$ & 0.803 & 0.818 \\
\hline SACRINDX & 12 & 0.518 & 0.951 & .915 (c) & 0.909 & 0.920 \\
\hline INNOHT & 12 & 0.951 & 0.326 & $.273(c)$ & 0.264 & 0.281 \\
\hline ILIACBR & 12 & 0.479 & 0.976 & $.953(c)$ & 0.949 & 0.957 \\
\hline PUBISL & 9 & 0.662 & 0.773 & $.697(\mathrm{c})$ & 0.688 & 0.706 \\
\hline ISCHIUML & 12 & 0.593 & 0.873 & $.820(c)$ & 0.813 & 0.828 \\
\hline BIILIACBR & 10 & 0.849 & 0.467 & $.400(c)$ & 0.390 & 0.409 \\
\hline ISCPUBINDX & 9 & 0.481 & 0.975 & $.951(c)$ & 0.947 & 0.955 \\
\hline FEMXL & 14 & 0.333 & 1.000 & $.999(c)$ & 0.999 & 1.000 \\
\hline FEMBL & 14 & 0.282 & 1.000 & $1.000(\mathrm{c})$ & 1.000 & 1.000 \\
\hline FEMSTAPD & 15 & 0.834 & 0.489 & $.432(\mathrm{c})$ & 0.422 & 0.441 \\
\hline FEMSTMLD & 15 & 0.662 & 0.773 & $.711(\mathrm{c})$ & 0.703 & 0.720 \\
\hline PLATYMINDX & 15 & 0.789 & 0.563 & $.501(c)$ & 0.491 & 0.511 \\
\hline FEMMIDADL & 15 & 0.894 & 0.402 & $.350(c)$ & 0.341 & 0.360 \\
\hline FEMMIDMD & 15 & 1.097 & 0.180 & $.145(c)$ & 0.138 & 0.152 \\
\hline FEMMIDSHFTINDX & 15 & 0.667 & 0.765 & $.702(c)$ & 0.693 & 0.711 \\
\hline FEMVHEDD & 14 & 0.947 & 0.331 & $.282(\mathrm{c})$ & 0.273 & 0.291 \\
\hline FEMHHEDD & 14 & 0.859 & 0.452 & $.396(c)$ & 0.387 & 0.406 \\
\hline FEMLCAPD & 14 & 0.603 & 0.861 & $.811(\mathrm{c})$ & 0.803 & 0.818 \\
\hline FEMMCAPD & 12 & 0.611 & 0.850 & $.796(c)$ & 0.788 & 0.804 \\
\hline FEMEPICB & 14 & 0.552 & 0.921 & $.882(\mathrm{c})$ & 0.875 & 0.888 \\
\hline FEMMIDCR & 15 & 0.602 & 0.862 & $.812(\mathrm{c})$ & 0.804 & 0.819 \\
\hline FEMRBSTINDX & 14 & 0.577 & 0.894 & $.851(\mathrm{c})$ & 0.844 & 0.857 \\
\hline TIBXL & 15 & 0.420 & 0.995 & $.988(c)$ & 0.986 & 0.990 \\
\hline TIBPEPBR & 15 & 0.565 & 0.907 & $.866(c)$ & 0.859 & 0.872 \\
\hline TIBDEPBR & 15 & 0.552 & 0.921 & $.884(c)$ & 0.877 & 0.890 \\
\hline TIBAPNFD & 15 & 0.620 & 0.837 & $.785(c)$ & 0.777 & 0.793 \\
\hline TIBMLNFD & 15 & 0.821 & 0.511 & $.451(c)$ & 0.441 & 0.461 \\
\hline PLATYCNINDX & 15 & 0.970 & 0.304 & $.257(c)$ & 0.249 & 0.266 \\
\hline TIBNUTFC & 15 & 0.760 & 0.610 & $.547(\mathrm{c})$ & 0.537 & 0.556 \\
\hline TIBFEMINDX & 14 & 0.595 & 0.870 & $.822(\mathrm{c})$ & 0.814 & 0.829 \\
\hline FIBXL & 6 & 0.635 & 0.815 & $.735(c)$ & 0.727 & 0.744 \\
\hline FIBMSXD & 6 & 0.519 & 0.950 & $.902(\mathrm{c})$ & 0.896 & 0.908 \\
\hline CALCXLN & 10 & 0.778 & 0.580 & $.511(\mathrm{c})$ & 0.502 & 0.521 \\
\hline CALCMBR & 10 & 0.927 & 0.356 & $.294(c)$ & 0.285 & 0.303 \\
\hline MT1MXHT & 8 & 0.621 & 0.836 & $.768(\mathrm{c})$ & 0.760 & 0.776 \\
\hline MT1AP & 8 & 0.714 & 0.687 & $.605(c)$ & 0.596 & 0.615 \\
\hline MT1ML & 8 & 0.740 & 0.644 & $.563(\mathrm{c})$ & 0.553 & 0.573 \\
\hline
\end{tabular}

c. Based on 10000 sampled tables 


\begin{tabular}{|c|c|c|c|c|c|c|}
\hline \multicolumn{7}{|c|}{ Pre-2000 BP Males } \\
\hline \multirow[b]{3}{*}{ Variables } & \multirow[b]{3}{*}{$\mathrm{N}$} & \multirow[b]{3}{*}{ K-S Z } & \multirow[b]{3}{*}{$\mathrm{p}$} & \multicolumn{3}{|c|}{ Monte Carlo } \\
\hline & & & & & \multicolumn{2}{|c|}{$95 \%$ C.I. } \\
\hline & & & & $\mathrm{p}$ & Lower Bound & Upper Bound \\
\hline$\overline{C L A V X L}$ & 8 & 0.384 & 0.998 & $.993(\mathrm{c})$ & 0.991 & 0.995 \\
\hline CLAVAPD & 9 & 0.892 & 0.404 & $.338(\mathrm{c})$ & 0.329 & 0.348 \\
\hline CLAVSID & 9 & 1.053 & 0.217 & $.171(\mathrm{c})$ & 0.163 & 0.178 \\
\hline CLAVRBSTINDX & 8 & 0.386 & 0.998 & $.993(\mathrm{c})$ & 0.991 & 0.994 \\
\hline SCAPXHT & 2 & 0.368 & 0.999 & $.999(c)$ & 0.999 & 1.000 \\
\hline SCAPXBR & 3 & 0.488 & 0.971 & $.926(\mathrm{c})$ & 0.921 & 0.931 \\
\hline SCAPSPIL & 5 & 0.828 & 0.500 & $.399(\mathrm{c})$ & 0.390 & 0.409 \\
\hline SCAPGCBR & 8 & 0.707 & 0.699 & $.611(\mathrm{c})$ & 0.601 & 0.621 \\
\hline SCAPGCHT & 8 & 0.473 & 0.979 & $.952(\mathrm{c})$ & 0.948 & 0.956 \\
\hline SCAPINDX & 2 & 0.368 & 0.999 & $.999(\mathrm{c})$ & 0.999 & 1.000 \\
\hline HUMXL & 10 & 0.669 & 0.762 & $.687(\mathrm{c})$ & 0.678 & 0.696 \\
\hline HUMMIDXD & 11 & 0.814 & 0.521 & $.453(c)$ & 0.443 & 0.463 \\
\hline HUMMIDND & 11 & 0.726 & 0.667 & $.594(\mathrm{c})$ & 0.584 & 0.603 \\
\hline HUMMIDSHFTINDX & 11 & 0.574 & 0.897 & $.842(\mathrm{c})$ & 0.835 & 0.849 \\
\hline HUMHEADD & 11 & 0.446 & 0.989 & $.973(\mathrm{c})$ & 0.970 & 0.976 \\
\hline HUMEPBR & 10 & 0.620 & 0.837 & $.767(\mathrm{c})$ & 0.759 & 0.775 \\
\hline HUMRBSTINDX & 10 & 0.518 & 0.951 & $.911(\mathrm{c})$ & 0.905 & 0.916 \\
\hline CLAHUMINDX & 7 & 0.705 & 0.703 & $.616(\mathrm{c})$ & 0.606 & 0.626 \\
\hline RADXL & 10 & 0.480 & 0.976 & $.949(\mathrm{c})$ & 0.945 & 0.953 \\
\hline RADHEADD & 11 & 0.800 & 0.545 & $.476(\mathrm{c})$ & 0.466 & 0.485 \\
\hline RADAPD & 10 & 1.325 & 0.060 & .040 (c) & 0.036 & 0.044 \\
\hline RADMLD & 10 & 0.774 & 0.587 & $.517(\mathrm{c})$ & 0.507 & 0.527 \\
\hline RADSHFTINDX & 10 & 0.828 & 0.500 & $.434(c)$ & 0.424 & 0.443 \\
\hline RADHUMINDX & 10 & 0.424 & 0.994 & $.980(\mathrm{c})$ & 0.977 & 0.982 \\
\hline ULNAXL & 6 & 0.496 & 0.966 & $.924(c)$ & 0.919 & 0.929 \\
\hline ULNAPHYL & 6 & 0.335 & 1.000 & $.999(\mathrm{c})$ & 0.998 & 1.000 \\
\hline ULNAXOBR & 9 & 0.647 & 0.796 & $.724(\mathrm{c})$ & 0.715 & 0.733 \\
\hline ULNANOBR & 9 & 0.703 & 0.706 & $.631(\mathrm{c})$ & 0.621 & 0.640 \\
\hline ULNAAPD & 8 & 0.611 & 0.849 & $.774(\mathrm{c})$ & 0.765 & 0.782 \\
\hline ULNAMLD & 7 & 0.679 & 0.746 & $.661(\mathrm{c})$ & 0.652 & 0.671 \\
\hline ULNASHFTINDX & 7 & 0.936 & 0.345 & $.277(\mathrm{c})$ & 0.268 & 0.286 \\
\hline SKTRNKHT & 3 & 0.482 & 0.975 & $.936(\mathrm{c})$ & 0.931 & 0.940 \\
\hline SACRANTH & 7 & 0.680 & 0.744 & .660 (c) & 0.651 & 0.669 \\
\hline SACRANTB & 10 & 0.782 & 0.573 & $.503(\mathrm{c})$ & 0.493 & 0.512 \\
\hline SACRS1BR & 10 & 0.452 & 0.987 & $.967(\mathrm{c})$ & 0.963 & 0.970 \\
\hline SACRINDX & 7 & 0.541 & 0.931 & $.878(\mathrm{c})$ & 0.872 & 0.885 \\
\hline INNOHT & 5 & 0.506 & 0.960 & $.912(\mathrm{c})$ & 0.906 & 0.917 \\
\hline ILIACBR & 8 & 0.630 & 0.823 & $.739(\mathrm{c})$ & 0.730 & 0.748 \\
\hline PUBISL & 6 & 0.416 & 0.995 & $.981(\mathrm{c})$ & 0.978 & 0.983 \\
\hline ISCHIUML & 8 & 1.024 & 0.245 & $.197(\mathrm{c})$ & 0.190 & 0.205 \\
\hline BIILIACBR & 3 & 0.499 & 0.965 & $.915(\mathrm{c})$ & 0.910 & 0.921 \\
\hline ISCPUBINDX & 6 & 0.504 & 0.961 & $.917(\mathrm{c})$ & 0.911 & 0.922 \\
\hline FEMXL & 9 & 0.563 & 0.909 & $.860(\mathrm{c})$ & 0.853 & 0.867 \\
\hline FEMBL & 9 & 0.584 & 0.885 & $.830(\mathrm{c})$ & 0.822 & 0.837 \\
\hline FEMSTAPD & 11 & 0.942 & 0.338 & $.286(\mathrm{c})$ & 0.277 & 0.294 \\
\hline FEMSTMLD & 11 & 0.487 & 0.972 & $.944(c)$ & 0.939 & 0.948 \\
\hline PLATYMINDX & 11 & 0.767 & 0.599 & $.527(\mathrm{c})$ & 0.517 & 0.537 \\
\hline FEMMIDADL & 12 & 0.490 & 0.970 & $.944(c)$ & 0.940 & 0.949 \\
\hline FEMMIDMD & 12 & 0.767 & 0.599 & $.529(\mathrm{c})$ & 0.519 & 0.538 \\
\hline FEMMIDSHFTINDX & 12 & 0.705 & 0.703 & $.629(\mathrm{c})$ & 0.620 & 0.639 \\
\hline FEMVHEDD & 10 & 0.690 & 0.727 & $.650(\mathrm{c})$ & 0.640 & 0.659 \\
\hline FEMHHEDD & 10 & 0.851 & 0.464 & $.399(\mathrm{c})$ & 0.389 & 0.408 \\
\hline FEMLCAPD & 10 & 0.454 & 0.986 & $.966(\mathrm{c})$ & 0.962 & 0.969 \\
\hline FEMMCAPD & 11 & 0.491 & 0.970 & $.941(\mathrm{c})$ & 0.936 & 0.945 \\
\hline FEMEPICB & 10 & 0.468 & 0.981 & $.958(\mathrm{c})$ & 0.954 & 0.961 \\
\hline FEMMIDCR & 12 & 0.606 & 0.857 & $.800(\mathrm{c})$ & 0.792 & 0.807 \\
\hline FEMRBSTINDX & 9 & 0.674 & 0.755 & $.679(\mathrm{c})$ & 0.670 & 0.688 \\
\hline TIBXL & 8 & 0.488 & 0.971 & $.939(\mathrm{c})$ & 0.934 & 0.944 \\
\hline TIBPEPBR & 8 & 0.630 & 0.822 & $.738(\mathrm{c})$ & 0.729 & 0.746 \\
\hline TIBDEPBR & 9 & 0.568 & 0.904 & $.854(\mathrm{c})$ & 0.847 & 0.860 \\
\hline TIBAPNFD & 10 & 0.564 & 0.908 & $.850(\mathrm{c})$ & 0.843 & 0.857 \\
\hline TIBMLNFD & 10 & 0.797 & 0.548 & $.477(\mathrm{c})$ & 0.467 & 0.487 \\
\hline PLATYCNINDX & 10 & 0.540 & 0.932 & $.885(c)$ & 0.878 & 0.891 \\
\hline TIBNUTFC & 10 & 0.459 & 0.985 & $.963(\mathrm{c})$ & 0.959 & 0.966 \\
\hline TIBFEMINDX & 7 & 0.653 & 0.787 & $.705(c)$ & 0.696 & 0.714 \\
\hline FIBXL & 3 & 0.531 & 0.941 & $.873(\mathrm{c})$ & 0.866 & 0.879 \\
\hline FIBMSXD & 5 & 0.509 & 0.958 & $.908(\mathrm{c})$ & 0.903 & 0.914 \\
\hline CALCXLN & 11 & 0.564 & 0.909 & $.855(c)$ & 0.848 & 0.862 \\
\hline CALCMBR & 11 & 0.615 & 0.843 & .779 (c) & 0.771 & 0.787 \\
\hline MT1MXHT & 9 & 0.447 & 0.988 & .969 (c) & 0.966 & 0.973 \\
\hline MT1AP & 10 & 0.665 & 0.769 & $.693(\mathrm{c})$ & 0.684 & 0.702 \\
\hline MT1ML & 10 & 0.730 & 0.660 & $.585(\mathrm{c})$ & 0.576 & 0.595 \\
\hline
\end{tabular}

c. Based on 10000 sampled tables 


\begin{tabular}{|c|c|c|c|c|c|c|}
\hline \multicolumn{7}{|c|}{ Post-2000 BP Males } \\
\hline \multirow[b]{3}{*}{ Variables } & \multirow[b]{3}{*}{$\mathrm{N}$} & \multirow[b]{3}{*}{ K-S Z } & \multirow[b]{3}{*}{$\mathrm{p}$} & \multicolumn{3}{|c|}{ Monte Carlo } \\
\hline & & & & & \multicolumn{2}{|c|}{ 95\% C.I. } \\
\hline & & & & $\mathrm{p}$ & Lower Bound & Upper Bound \\
\hline$\overline{C L A V X L}$ & 5 & 0.811 & 0.526 & $.415(\mathrm{c})$ & 0.406 & 0.425 \\
\hline CLAVAPD & 6 & 0.503 & 0.962 & $.919(c)$ & 0.914 & 0.924 \\
\hline CLAVSID & 6 & 0.755 & 0.619 & $.524(c)$ & 0.514 & 0.533 \\
\hline CLAVRBSTINDX & 5 & 0.597 & 0.868 & $.791(c)$ & 0.783 & 0.799 \\
\hline SCAPXHT & 5 & 0.782 & 0.573 & $.464(c)$ & 0.454 & 0.474 \\
\hline SCAPXBR & 5 & 0.568 & 0.904 & $.837(\mathrm{c})$ & 0.830 & 0.844 \\
\hline SCAPSPIL & 5 & 0.744 & 0.637 & $.534(\mathrm{c})$ & 0.524 & 0.543 \\
\hline SCAPGCBR & 8 & 0.871 & 0.434 & $.360(c)$ & 0.350 & 0.369 \\
\hline SCAPGCHT & 8 & 0.597 & 0.869 & $.801(c)$ & 0.794 & 0.809 \\
\hline SCAPINDX & 5 & 0.464 & 0.982 & $.953(\mathrm{c})$ & 0.948 & 0.957 \\
\hline HUMXL & 7 & 0.506 & 0.960 & $.919(\mathrm{c})$ & 0.913 & 0.924 \\
\hline HUMMIDXD & 8 & 0.917 & 0.369 & $.301(c)$ & 0.292 & 0.310 \\
\hline HUMMIDND & 8 & 0.635 & 0.815 & $.738(\mathrm{c})$ & 0.730 & 0.747 \\
\hline HUMMIDSHFTINDX & 8 & 0.507 & 0.959 & $.922(\mathrm{c})$ & 0.917 & 0.928 \\
\hline HUMHEADD & 7 & 0.451 & 0.987 & $.964(c)$ & 0.960 & 0.967 \\
\hline HUMEPBR & 7 & 0.490 & 0.970 & $.936(c)$ & 0.931 & 0.941 \\
\hline HUMRBSTINDX & 7 & 0.825 & 0.503 & $.424(c)$ & 0.414 & 0.434 \\
\hline CLAHUMINDX & 5 & 0.489 & 0.971 & $.933(\mathrm{c})$ & 0.928 & 0.938 \\
\hline RADXL & 7 & 0.387 & 0.998 & $.992(c)$ & 0.990 & 0.993 \\
\hline RADHEADD & 7 & 0.619 & 0.839 & $.764(\mathrm{c})$ & 0.756 & 0.773 \\
\hline RADAPD & 8 & 0.707 & 0.699 & $.614(\mathrm{c})$ & 0.605 & 0.624 \\
\hline RADMLD & 8 & 0.623 & 0.832 & $.760(\mathrm{c})$ & 0.752 & 0.768 \\
\hline RADSHFTINDX & 8 & 0.947 & 0.332 & $.270(\mathrm{c})$ & 0.262 & 0.279 \\
\hline RADHUMINDX & 7 & 0.994 & 0.277 & $.214(\mathrm{c})$ & 0.206 & 0.222 \\
\hline ULNAXL & 7 & 0.397 & 0.997 & $.989(\mathrm{c})$ & 0.987 & 0.991 \\
\hline ULNAPHYL & 7 & 0.335 & 1.000 & $.999(\mathrm{c})$ & 0.999 & 1.000 \\
\hline ULNAXOBR & 7 & 0.633 & 0.817 & $.739(\mathrm{c})$ & 0.730 & 0.747 \\
\hline ULNANOBR & 7 & 0.473 & 0.979 & $.949(\mathrm{c})$ & 0.944 & 0.953 \\
\hline ULNAAPD & 7 & 0.579 & 0.891 & $.830(\mathrm{c})$ & 0.822 & 0.837 \\
\hline ULNAMLD & 7 & 0.670 & 0.761 & $.676(\mathrm{c})$ & 0.667 & 0.685 \\
\hline ULNASHFTINDX & 7 & 0.344 & 1.000 & $.999(c)$ & 0.998 & 0.999 \\
\hline SKTRNKHT & 4 & 0.782 & 0.574 & $.472(\mathrm{c})$ & 0.463 & 0.482 \\
\hline SACRANTH & 7 & 0.552 & 0.920 & $.865(\mathrm{c})$ & 0.858 & 0.871 \\
\hline SACRANTB & 6 & 0.489 & 0.970 & $.931(\mathrm{c})$ & 0.926 & 0.936 \\
\hline SACRS1BR & 8 & 0.509 & 0.958 & $.920(c)$ & 0.915 & 0.925 \\
\hline SACRINDX & 5 & 0.661 & 0.775 & $.684(\mathrm{c})$ & 0.675 & 0.693 \\
\hline INNOHT & 7 & 0.433 & 0.992 & $.975(\mathrm{c})$ & 0.971 & 0.978 \\
\hline ILIACBR & 8 & 0.963 & 0.312 & $.252(\mathrm{c})$ & 0.244 & 0.261 \\
\hline PUBISL & 7 & 0.814 & 0.521 & $.442(c)$ & 0.432 & 0.452 \\
\hline ISCHIUML & 7 & 0.483 & 0.974 & $.941(\mathrm{c})$ & 0.936 & 0.946 \\
\hline BIILIACBR & 5 & 0.648 & 0.795 & $.707(\mathrm{c})$ & 0.698 & 0.716 \\
\hline ISCPUBINDX & 7 & 0.613 & 0.846 & $.775(c)$ & 0.767 & 0.783 \\
\hline FEMXL & 8 & 0.502 & 0.963 & $.928(\mathrm{c})$ & 0.923 & 0.933 \\
\hline FEMBL & 7 & 0.523 & 0.947 & $.901(\mathrm{c})$ & 0.895 & 0.906 \\
\hline FEMSTAPD & 8 & 0.710 & 0.694 & $.609(\mathrm{c})$ & 0.599 & 0.619 \\
\hline FEMSTMLD & 8 & 0.623 & 0.832 & $.760(\mathrm{c})$ & 0.751 & 0.768 \\
\hline PLATYMINDX & 8 & 0.738 & 0.647 & $.559(\mathrm{c})$ & 0.550 & 0.569 \\
\hline FEMMIDADL & 8 & 0.508 & 0.958 & $.921(\mathrm{c})$ & 0.915 & 0.926 \\
\hline FEMMIDMD & 8 & 0.707 & 0.699 & $.614(\mathrm{c})$ & 0.605 & 0.624 \\
\hline FEMMIDSHFTINDX & 8 & 0.768 & 0.598 & $.513(\mathrm{c})$ & 0.504 & 0.523 \\
\hline FEMVHEDD & 8 & 0.686 & 0.734 & $.650(\mathrm{c})$ & 0.641 & 0.660 \\
\hline FEMHHEDD & 8 & 0.432 & 0.992 & $.981(\mathrm{c})$ & 0.978 & 0.983 \\
\hline FEMLCAPD & 7 & 0.803 & 0.540 & $.458(c)$ & 0.448 & 0.468 \\
\hline FEMMCAPD & 7 & 0.659 & 0.778 & $.696(\mathrm{c})$ & 0.687 & 0.705 \\
\hline FEMEPICB & 8 & 0.599 & 0.866 & $.798(c)$ & 0.790 & 0.806 \\
\hline FEMMIDCR & 8 & 0.948 & 0.330 & $.269(\mathrm{c})$ & 0.260 & 0.277 \\
\hline FEMRBSTINDX & 7 & 0.374 & 0.999 & $.995(c)$ & 0.993 & 0.996 \\
\hline TIBXL & 7 & 0.487 & 0.971 & $.938(\mathrm{c})$ & 0.933 & 0.943 \\
\hline TIBPEPBR & 7 & 0.425 & 0.994 & $.978(\mathrm{c})$ & 0.975 & 0.981 \\
\hline TIBDEPBR & 8 & 0.771 & 0.593 & $.508(c)$ & 0.498 & 0.518 \\
\hline TIBAPNFD & 7 & 0.670 & 0.761 & $.676(\mathrm{c})$ & 0.667 & 0.685 \\
\hline TIBMLNFD & 7 & 0.500 & 0.964 & $.925(c)$ & 0.920 & 0.930 \\
\hline PLATYCNINDX & 7 & 0.460 & 0.984 & $.958(c)$ & 0.954 & 0.962 \\
\hline TIBNUTFC & 7 & 0.719 & 0.679 & $.587(\mathrm{c})$ & 0.578 & 0.597 \\
\hline TIBFEMINDX & 7 & 0.581 & 0.888 & $.825(c)$ & 0.818 & 0.833 \\
\hline FIBXL & 5 & 0.497 & 0.966 & $.926(c)$ & 0.920 & 0.931 \\
\hline FIBMSXD & 6 & 1.205 & 0.110 & $.074(c)$ & 0.069 & 0.079 \\
\hline CALCXLN & 7 & 0.625 & 0.829 & $.755(c)$ & 0.746 & 0.763 \\
\hline CALCMBR & 7 & 0.623 & 0.832 & $.758(c)$ & 0.750 & 0.767 \\
\hline MT1MXHT & 5 & 0.508 & 0.958 & $.912(\mathrm{c})$ & 0.906 & 0.918 \\
\hline MT1AP & 5 & 0.564 & 0.908 & $.842(\mathrm{c})$ & 0.835 & 0.849 \\
\hline MT1ML & 5 & 0.305 & 1.000 & $1.000(\mathrm{c})$ & 1.000 & 1.000 \\
\hline
\end{tabular}

c. Based on 10000 sampled tables 
Appendix 15. Levene's test for homogeneity of variances for the craniometric variable set

\begin{tabular}{|c|c|c|c|c|}
\hline Variables & Levene Statistic & df1 & df2 & p \\
\hline$\overline{\mathrm{GOL}}$ & 0.691 & 3 & 41 & 0.563 \\
\hline NOL & 0.337 & 3 & 41 & 0.799 \\
\hline BNL & 1.818 & 3 & 38 & 0.160 \\
\hline $\mathrm{BBH}$ & 0.297 & 3 & 39 & 0.828 \\
\hline XCB & 1.877 & 3 & 42 & 0.148 \\
\hline XFB & 2.959 & 3 & 38 & 0.044 \\
\hline WFB & 2.707 & 3 & 42 & 0.057 \\
\hline ZYB & 2.328 & 3 & 28 & 0.096 \\
\hline AUB & 1.402 & 3 & 39 & 0.257 \\
\hline ASB & 3.291 & 3 & 46 & 0.029 \\
\hline BPL & 1.379 & 3 & 37 & 0.264 \\
\hline $\mathrm{NPH}$ & 3.467 & 3 & 45 & 0.024 \\
\hline $\mathrm{NLH}$ & 1.809 & 3 & 44 & 0.160 \\
\hline JUB & 0.777 & 3 & 42 & 0.513 \\
\hline NLB & 0.272 & 3 & 46 & 0.845 \\
\hline MAB & 1.283 & 3 & 45 & 0.292 \\
\hline MAL & 1.105 & 3 & 38 & 0.359 \\
\hline $\mathrm{MDH}$ & 0.099 & 3 & 50 & 0.960 \\
\hline $\mathrm{OBH}$ & 2.585 & 3 & 45 & 0.065 \\
\hline OBB & 1.332 & 3 & 45 & 0.276 \\
\hline DKB & 0.901 & 3 & 46 & 0.448 \\
\hline NDS & 3.069 & 3 & 36 & 0.040 \\
\hline WNB & 0.984 & 3 & 37 & 0.411 \\
\hline SIS & 3.734 & 3 & 36 & 0.020 \\
\hline ZMB & 0.517 & 3 & 41 & 0.673 \\
\hline SSS & 1.535 & 3 & 38 & 0.221 \\
\hline FMB & 0.731 & 3 & 43 & 0.539 \\
\hline NAS & 0.572 & 3 & 42 & 0.637 \\
\hline EKB & 0.530 & 3 & 42 & 0.664 \\
\hline DKS & 0.480 & 3 & 38 & 0.698 \\
\hline IML & 0.028 & 3 & 37 & 0.993 \\
\hline XML-Z & 0.898 & 3 & 39 & 0.451 \\
\hline MLS & 1.468 & 3 & 36 & 0.240 \\
\hline WMH & 3.399 & 3 & 46 & 0.025 \\
\hline GLS & 6.141 & 3 & 44 & 0.001 \\
\hline STB & 2.244 & 3 & 37 & 0.099 \\
\hline FRC & 2.045 & 3 & 43 & 0.122 \\
\hline FRS & 0.405 & 3 & 43 & 0.750 \\
\hline FRF & 1.356 & 3 & 43 & 0.269 \\
\hline PAC & 0.379 & 3 & 46 & 0.769 \\
\hline PAS & 2.677 & 3 & 45 & 0.058 \\
\hline PAF & 1.004 & 3 & 45 & 0.400 \\
\hline OCC & 0.489 & 3 & 39 & 0.692 \\
\hline OCS & 0.366 & 3 & 39 & 0.778 \\
\hline OCF & 3.623 & 3 & 39 & 0.021 \\
\hline FOL & 1.614 & 3 & 36 & 0.203 \\
\hline FOB & 0.558 & 3 & 40 & 0.646 \\
\hline UFBR & 0.887 & 3 & 39 & 0.456 \\
\hline GNI & 1.631 & 3 & 47 & 0.195 \\
\hline HML & 0.545 & 3 & 49 & 0.654 \\
\hline TML & 0.081 & 3 & 49 & 0.970 \\
\hline GOG & 0.807 & 3 & 40 & 0.498 \\
\hline CDL & 1.544 & 3 & 32 & 0.222 \\
\hline WRB & 1.158 & 3 & 43 & 0.337 \\
\hline XRH & 1.387 & 3 & 41 & 0.260 \\
\hline MXML & 1.118 & 3 & 39 & 0.353 \\
\hline MAN & 3.893 & 3 & 42 & 0.015 \\
\hline
\end{tabular}


Appendix 16. Levene's test for homogeneity of variances for the maximum odontometric variable set

\begin{tabular}{|c|c|c|c|c|}
\hline Variables $^{1}$ & Levene Statistic & df1 & df2 & $\mathbf{p}$ \\
\hline UI1MBL & * & & & \\
\hline UI2MBL & * & & & \\
\hline UCMBL & 3.416 & 3 & 23 & 0.034 \\
\hline UP1MBL & 0.157 & 3 & 21 & 0.924 \\
\hline UP1MMD & * & & & \\
\hline UP1MCRA & * & & & \\
\hline UP2MBL & 0.037 & 3 & 22 & 0.990 \\
\hline UP2MMD & * & & & \\
\hline UP2MCRA & * & & & \\
\hline UM1MBL & 0.403 & 3 & 19 & 0.752 \\
\hline UM2MBL & 0.883 & 3 & 24 & 0.464 \\
\hline UM3MBL & 0.261 & 2 & 16 & 0.774 \\
\hline UM3MMD & * & & & \\
\hline UM3MCRA & * & & & \\
\hline LIIMBL & 3.071 & 3 & 15 & 0.060 \\
\hline LI2MBL & 3.894 & 3 & 20 & 0.024 \\
\hline LCMBL & 1.190 & 3 & 24 & 0.334 \\
\hline LCMMD & * & & & \\
\hline LCMCRA & * & & & \\
\hline LP1MBL & 1.062 & 3 & 28 & 0.381 \\
\hline LP1MMD & * & & & \\
\hline LP1MCRA & * & & & \\
\hline LP2MBL & 1.489 & 3 & 23 & 0.244 \\
\hline LP2MMD & * & & & \\
\hline LP2MCRA & * & & & \\
\hline LM1MBL & 0.437 & 2 & 13 & 0.655 \\
\hline LM2MBL & 0.639 & 3 & 24 & 0.597 \\
\hline
\end{tabular}

* There are not enough unique spread/level pairs to compute the Levene statistic.

1. Levene statistic not calculated for maximum odontometric variables not listed due to insufficient numbers of available cases. 
Appendix 17. Levene's test for homogeneity of variances for the cervical odontometric variable set

\begin{tabular}{|c|c|c|c|c|}
\hline Variables & Levene Statistic & df1 & df2 & $\mathbf{p}$ \\
\hline UI1CBL & * & & & \\
\hline UI1CMD & 1.520 & 3 & 19 & 0.242 \\
\hline UI1CCRA & * & & & \\
\hline UI2CBL & 0.798 & 3 & 19 & 0.510 \\
\hline UI2CMD & 0.580 & 3 & 20 & 0.635 \\
\hline UI2CCRA & 0.304 & 3 & 18 & 0.822 \\
\hline UCCBL & 3.128 & 3 & 29 & 0.041 \\
\hline UCCMD & 1.175 & 3 & 28 & 0.337 \\
\hline UCCCRA & 1.621 & 3 & 27 & 0.208 \\
\hline UP1CBL & 0.122 & 3 & 32 & 0.947 \\
\hline UP1CMD & 0.298 & 3 & 32 & 0.827 \\
\hline UP1CCRA & 0.821 & 3 & 30 & 0.492 \\
\hline UP2CBL & 1.865 & 3 & 25 & 0.161 \\
\hline UP2CMD & 1.368 & 3 & 28 & 0.273 \\
\hline UP2CCRA & 0.184 & 3 & 23 & 0.906 \\
\hline UM1CBL & 2.297 & 3 & 30 & 0.098 \\
\hline UM1CMD & 1.700 & 3 & 26 & 0.192 \\
\hline UMICCRA & 4.609 & 3 & 24 & 0.011 \\
\hline UM2CBL & 2.482 & 3 & 36 & 0.076 \\
\hline UM2CMD & 0.485 & 3 & 27 & 0.695 \\
\hline UM2CCRA & 0.583 & 3 & 25 & 0.632 \\
\hline UM3CBL & 0.859 & 3 & 23 & 0.476 \\
\hline UM3CMD & 0.313 & 1 & 13 & 0.585 \\
\hline UM3CCRA & 0.661 & 1 & 11 & 0.433 \\
\hline LI1CBL & * & & & \\
\hline LI1CMD & 3.582 & 3 & 20 & 0.032 \\
\hline LI1CCRA & * & & & \\
\hline LI2CBL & 5.216 & 3 & 22 & 0.007 \\
\hline LI2CMD & 0.822 & 3 & 25 & 0.494 \\
\hline LI2CCRA & 3.290 & 3 & 21 & 0.041 \\
\hline LCCBL & 0.485 & 3 & 29 & 0.695 \\
\hline LCCMD & 1.175 & 3 & 29 & 0.336 \\
\hline LCCCRA & 0.954 & 3 & 28 & 0.428 \\
\hline LP1CBL & 0.167 & 3 & 38 & 0.918 \\
\hline LP1CMD & 0.795 & 3 & 37 & 0.504 \\
\hline LP1CCRA & 0.402 & 3 & 37 & 0.753 \\
\hline LP2CBL & 1.569 & 3 & 33 & 0.215 \\
\hline LP2CMD & 1.105 & 3 & 35 & 0.360 \\
\hline LP2CCRA & 1.868 & 3 & 33 & 0.154 \\
\hline LM1CBL & 2.530 & 3 & 30 & 0.076 \\
\hline LM1CMD & 2.766 & 3 & 27 & 0.061 \\
\hline LM1CCRA & 3.410 & 3 & 24 & 0.034 \\
\hline LM2CBL & 1.322 & 3 & 38 & 0.281 \\
\hline LM2CMD & 1.326 & 3 & 32 & 0.283 \\
\hline LM2CCRA & 2.542 & 3 & 29 & 0.076 \\
\hline
\end{tabular}

* There are not enough unique spread/level pairs to compute the Levene statistic 
Appendix 18. Levene's test for homogeneity of variances for the postcranial metric variable set

\begin{tabular}{|c|c|c|c|c|}
\hline Postcranial Variable & Levene Statistic & df1 & df2 & $\mathbf{p}$ \\
\hline CLAVXL & 0.208 & 3 & 35 & 0.890 \\
\hline CLAVAPD & 3.091 & 3 & 37 & 0.039 \\
\hline CLAVSID & 2.189 & 3 & 38 & 0.105 \\
\hline CLAVRBSTINDX & 1.105 & 3 & 35 & 0.360 \\
\hline SCAPXHT & 0.855 & 1 & 12 & 0.373 \\
\hline SCAPXBR & 2.090 & 1 & 18 & 0.165 \\
\hline SCAPSPIL & 0.805 & 3 & 26 & 0.503 \\
\hline SCAPGCBR & 1.283 & 3 & 40 & 0.293 \\
\hline SCAPGCHT & 0.436 & 3 & 40 & 0.729 \\
\hline SCAPINDX & 2.543 & 1 & 11 & 0.139 \\
\hline HUMXL & 0.903 & 3 & 40 & 0.448 \\
\hline HUMMIDXD & 3.560 & 3 & 44 & 0.022 \\
\hline HUMMIDND & 1.109 & 3 & 44 & 0.356 \\
\hline HUMMIDSHFTINDX & 0.589 & 3 & 44 & 0.625 \\
\hline HUMHEADD & 4.970 & 3 & 42 & 0.005 \\
\hline HUMEPBR & 2.527 & 3 & 41 & 0.071 \\
\hline HUMRBSTINDX & 1.753 & 3 & 40 & 0.172 \\
\hline CLAHUMINDX & 2.095 & 3 & 32 & 0.120 \\
\hline RADXL & 1.404 & 3 & 36 & 0.258 \\
\hline RADHEADD & 1.328 & 3 & 39 & 0.279 \\
\hline RADAPD & 1.395 & 3 & 42 & 0.258 \\
\hline RADMLD & 1.607 & 3 & 42 & 0.202 \\
\hline RADSHFTINDX & 0.982 & 3 & 42 & 0.410 \\
\hline RADHUMINDX & 0.525 & 3 & 34 & 0.668 \\
\hline ULNAXL & 1.784 & 3 & 33 & 0.169 \\
\hline ULNAPHYL & 1.511 & 3 & 31 & 0.231 \\
\hline ULNAXOBR & 2.755 & 3 & 37 & 0.056 \\
\hline ULNANOBR & 2.184 & 3 & 38 & 0.106 \\
\hline ULNAAPD & 0.588 & 3 & 37 & 0.627 \\
\hline ULNAMLD & 2.907 & 3 & 36 & 0.048 \\
\hline ULNASHFTINDX & 0.613 & 3 & 36 & 0.611 \\
\hline SACRANTH & 0.063 & 3 & 29 & 0.979 \\
\hline SACRANTB & 2.689 & 3 & 34 & 0.062 \\
\hline SACRS1BR & 1.811 & 3 & 37 & 0.162 \\
\hline SACRINDX & 0.450 & 3 & 26 & 0.719 \\
\hline INNOHT & 0.866 & 3 & 27 & 0.471 \\
\hline ILIACBR & 1.056 & 3 & 33 & 0.381 \\
\hline PUBISL & 0.492 & 3 & 24 & 0.691 \\
\hline ISCHIUML & 0.999 & 3 & 31 & 0.406 \\
\hline BIILIACBR & 1.829 & 1 & 14 & 0.198 \\
\hline ISCPUBINDX & 2.265 & 3 & 23 & 0.108 \\
\hline FEMXL & 0.756 & 3 & 40 & 0.525 \\
\hline FEMBL & 1.091 & 3 & 39 & 0.365 \\
\hline FEMSTAPD & 2.006 & 3 & 45 & 0.127 \\
\hline FEMSTMLD & 1.557 & 3 & 45 & 0.213 \\
\hline PLATYMINDX & 0.399 & 3 & 45 & 0.754 \\
\hline FEMMIDADL & 2.192 & 3 & 46 & 0.102 \\
\hline FEMMIDMD & 3.230 & 3 & 46 & 0.031 \\
\hline FEMMIDSHFTINDX & 1.098 & 3 & 46 & 0.360 \\
\hline FEMVHEDD & 1.245 & 3 & 40 & 0.306 \\
\hline FEMHHEDD & 0.956 & 3 & 42 & 0.423 \\
\hline FEMLCAPD & 1.106 & 3 & 41 & 0.358 \\
\hline FEMMCAPD & 0.308 & 3 & 38 & 0.819 \\
\hline FEMEPICB & 2.106 & 3 & 40 & 0.115 \\
\hline FEMMIDCR & 0.438 & 3 & 46 & 0.727 \\
\hline FEMRBSTINDX & 1.178 & 3 & 39 & 0.330 \\
\hline TIBXL & 0.163 & 3 & 37 & 0.921 \\
\hline TIBPEPBR & 0.264 & 3 & 37 & 0.851 \\
\hline TIBDEPBR & 0.536 & 3 & 41 & 0.660 \\
\hline TIBAPNFD & 1.637 & 3 & 41 & 0.196 \\
\hline TIBMLNFD & 0.452 & 3 & 41 & 0.717 \\
\hline PLATYCNINDX & 0.977 & 3 & 41 & 0.413 \\
\hline TIBNUTFC & 1.190 & 3 & 41 & 0.326 \\
\hline TIBFEMINDX & 0.259 & 3 & 34 & 0.855 \\
\hline FIBXL & 0.044 & 1 & 10 & 0.838 \\
\hline FIBMSXD & 2.523 & 3 & 22 & 0.084 \\
\hline CALCXLN & 0.420 & 3 & 34 & 0.740 \\
\hline CALCMBR & 0.807 & 3 & 34 & 0.499 \\
\hline MT1MXHT & 0.722 & 3 & 27 & 0.548 \\
\hline MT1AP & 6.351 & 3 & 28 & 0.002 \\
\hline MT1ML & 0.481 & 3 & 27 & 0.698 \\
\hline
\end{tabular}

